\title{
Dietary Patterns during Pregnancy and Gestational Weight Gain: A Systematic Review
}

\author{
2020 Dietary Guidelines Advisory Committee, \\ Pregnancy and Lactation Subcommittee \\ Published date: July 15, 2020
}

Nutrition Evidence Systematic Review

Center for Nutrition Policy and Promotion

Food and Nutrition Service

U.S. Department of Agriculture

Braddock Metro Center II

1320 Braddock Place

Alexandria, Virginia 22314 
This systematic review was conducted by the 2020 Dietary Guidelines Advisory Committee in collaboration with the Nutrition Evidence Systematic Review (NESR) team at the Center for Nutrition Policy and Promotion, Food and Nutrition Service, U.S. Department of Agriculture (USDA). All systematic reviews from the 2020 Advisory Committee Project are available on the NESR website: https://nesr.usda.gov/2020-dietaryguidelines-advisory-committee-systematic-reviews.

Conclusion statements drawn as part of this systematic review describe the state of science related to the specific question examined. Conclusion statements do not draw implications, and should not be interpreted as dietary guidance. This portfolio provides the complete documentation for this systematic review. A summary of this review is included in the 2020 Advisory Committee's Scientific Report available at www.DietaryGuidelines.gov.

The contents of this document may be used and reprinted without permission. Endorsements by NESR, the Center for Nutrition Policy and Promotion, the Food and Nutrition Service, or the USDA of derivative products developed from this work may not be stated or implied.

Suggested citation for this systematic review: 2020 Dietary Guidelines Advisory Committee and Nutrition Evidence Systematic Review Team. Dietary Patterns during Pregnancy and Gestational Weight Gain: A Systematic Review. 2020 Dietary Guidelines Advisory Committee Project. Alexandria, VA: U.S. Department of Agriculture, Food and Nutrition Service, Center for Nutrition Policy and Promotion, July 2020. Available at: https://nesr.usda.gov/2020-dietary-guidelines-advisory-committee-systematic-reviews.

Related citation: Dietary Guidelines Advisory Committee. 2020. Scientific Report of the 2020 Dietary Guidelines Advisory Committee: Advisory Report to the Secretary of Agriculture and the Secretary of Health and Human Services. U.S. Department of Agriculture, Agricultural Research Service, Washington, DC.

In accordance with Federal civil rights law and USDA civil rights regulations and policies, the USDA, its Agencies, offices, and employees, and institutions participating in or administering USDA programs are prohibited from discriminating based on race, color, national origin, religion, sex, gender identity (including gender expression), sexual orientation, disability, age, marital status, family/parental status, income derived from a public assistance program, political beliefs, or reprisal or retaliation for prior civil rights activity, in any program or activity conducted or funded by USDA (not all bases apply to all programs). Remedies and complaint filing deadlines vary by program or incident.

Persons with disabilities who require alternative means of communication for program information (e.g., Braille, large print, audiotape, American Sign Language, etc.) should contact the responsible Agency or USDA's TARGET Center at (202) 720-2600 (voice and TTY) or contact USDA through the Federal Relay Service at (800) 877-8339. Additionally, program information may be made available in languages other than English.

To file a program discrimination complaint, complete the USDA Program Discrimination Complaint Form, AD3027, found online at How to File a Program Discrimination Complaint and at any USDA office or write a letter addressed to USDA and provide in the letter all of the information requested in the form. To request a copy of the complaint form, call (866) 632-9992. Submit your completed form or letter to USDA by: (1) mail:

U.S. Department of Agriculture, Office of the Assistant Secretary for Civil Rights, 1400 Independence Avenue, SW, Washington, D.C. 20250-9410; (2) fax: (202) 690-7442; or (3) email: program.intake@usda.gov.

USDA is an equal opportunity provider, employer, and lender. 


\section{ACKNOWLEDGEMENTS}

Pregnancy and Lactation Subcommittee:

- Sharon Donovan, PhD, RD, University of Illinois, Urbana-Champaign, Subcommittee Chair

- Kathryn Dewey, PhD, University of California, Davis

- Rachel Novotny, PhD, RDN, LD, University of Hawaii

- Jamie Stang, PhD, MPH, RD, University of Minnesota

- Elsie Taveras, MD, MPH, Massachusetts General Hospital, Harvard Medical School, and Harvard T.H. Chan School of Public Health

- Ronald Kleinman, MD, Massachusetts General Hospital, Harvard Medical School, Vice-Chair of the 2020 Dietary Guidelines Advisory Committee

\section{Nutrition Evidence Systematic Review (NESR) Team:}

- Ramkripa Raghavan, DrPH, MPH, MSc, Analyst, Panum Group

- Julie Nevins, PhD, Analyst, Panum Groupi

- Sara Scinto-Madonich, MS, Analyst, Panum Groupi

- Julia H. Kim, PhD, MPH, RD, Analyst, Panum Groupi

- Nancy Terry, MS, MLS, Biomedical Librarian, National Institutes of Health (NIH) Library, U.S. Department of Health and Human Services (HHS)

- Gisela Butera, MLIS, MEd, Systematic Review Librarian, Panum Groupi

- Julie Obbagy, PhD, RD, Project Lead, Office of Nutrition Guidance and Analysis (ONGA), Center for Nutrition Policy and Promotion (CNPP), Food and Nutrition Service (FNS), U.S. Department of Agriculture (USDA)

\section{Federal Liaisons:}

- Jean Altman, MS, ONGA, CNPP, FNS, USDA

- Meghan Adler, MS, RDN, ONGA, CNPP, FNS, USDA

- Jenna Fahle, MSPH, RDN, ONGA, CNPP, FNS, USDA (08/2019-11/2019)

\section{Project Leadership:}

- Eve Essery Stoody, PhD, Designated Federal Officer and Director, ONGA, CNPP, FNS, USDA

- Janet de Jesus, MS, RD, Nutrition Advisor, Office of Disease Prevention and Health Promotion, Office of the Assistant Secretary for Health, HHS

USDA and HHS implemented a process to identify topics and scientific questions to be examined by the 2020 Dietary Guidelines Advisory Committee. The Committee conducted its review of evidence in subcommittees for discussion by the full Committee during its public meetings. The role of the Committee members involved establishing all aspects of the protocol, which presented the plan for how they would examine the scientific evidence, including the inclusion and exclusion criteria;

i Under contract with the Food and Nutrition Service, United States Department of Agriculture. 
reviewing all studies that met the criteria they set; deliberating on the body of evidence for each question; and writing and grading the conclusion statements to be included in the scientific report the 2020 Committee submitted to USDA and HHS. The NESR team with assistance from Federal Liaisons and Project Leadership, supported the Committee by facilitating, executing, and documenting the work necessary to ensure the reviews were completed in accordance with NESR methodology. More information about the 2020 Dietary Guidelines Advisory Committee, including the process used to identify topics and questions, can be found at www. DietaryGuidelines.gov. More information about NESR can be found at NESR.usda.gov.

The Committee and NESR staff thank USDA's Agricultural Research Service for coordinating the peer review of this systematic review, and the Federal scientist peer reviewers for their time and expertise.

FUNDING SOURCE: United States Department of Agriculture, Food and Nutrition Service, Center for Nutrition Policy and Promotion, Alexandria, VA 


\section{TABLE OF CONTENTS}

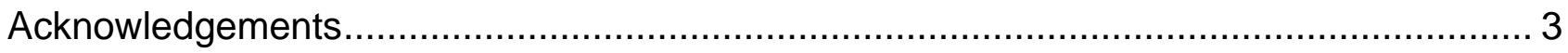

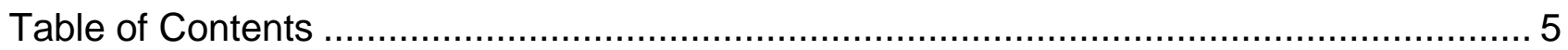

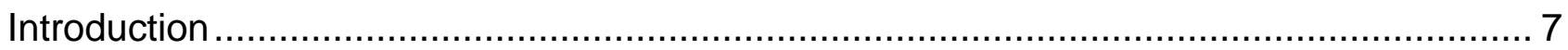

What is the relationship between dietary patterns consumed during pregnancy and

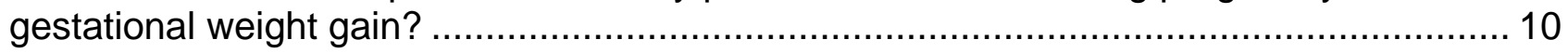

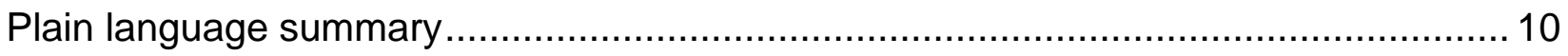

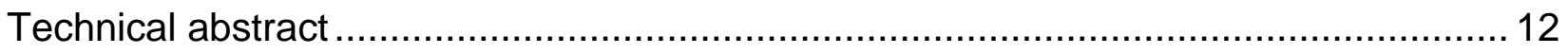

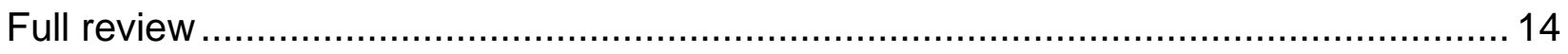

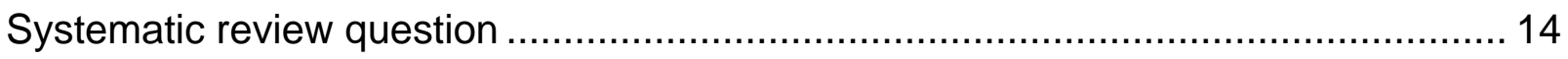

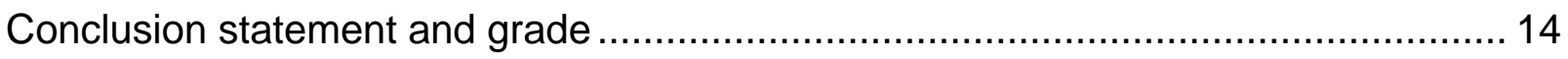

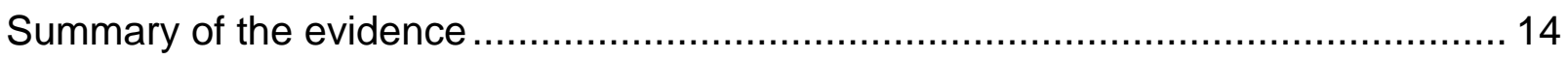

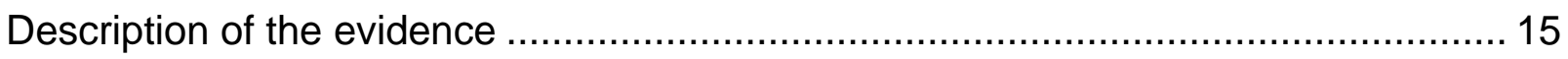

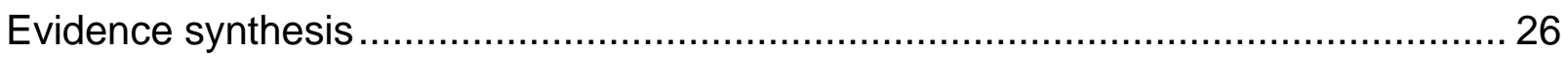

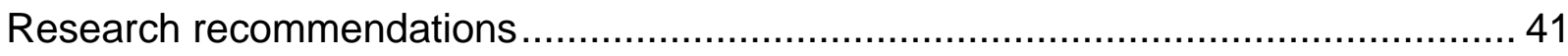

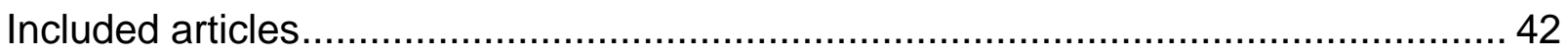

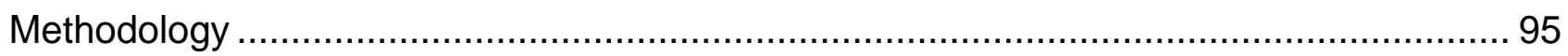

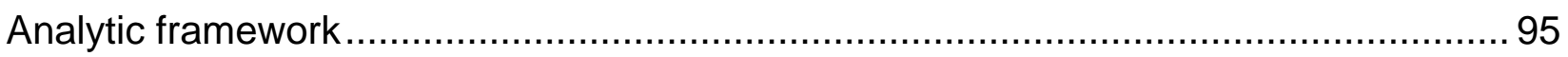

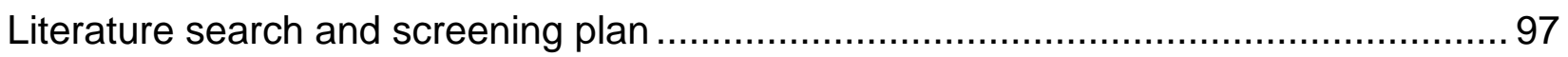

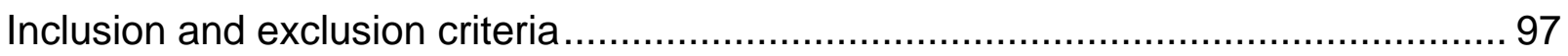

Electronic databases and search terms.......................................................... 100

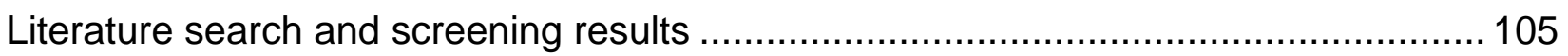

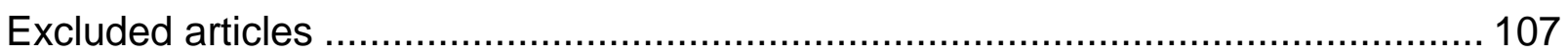

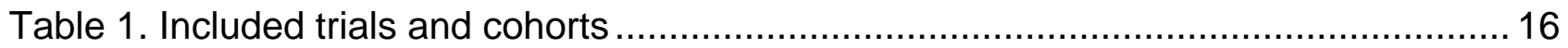

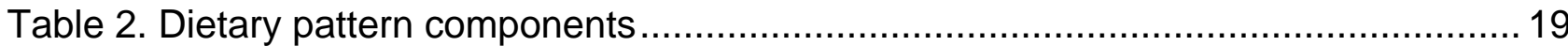

Table 3. Description of evidence on the relationship between dietary patterns during pregnancy and gestational weight gain................................................................... 45 Table 4. Description of evidence on the relationship between diets based on macronutrient distribution during pregnancy and gestational weight gain ........................................ 90 Table 5. Risk of bias for randomized controlled trials examining dietary patterns during pregnancy and gestational weight gain ................................................................... 93 Table 6. Risk of bias for observational studies examining dietary patterns during pregnancy

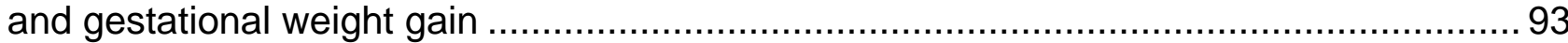

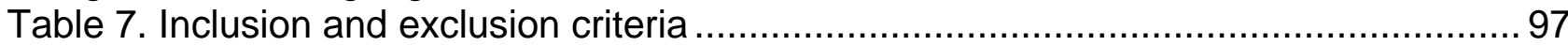

Table 8. Articles excluded after full text screening with rationale for exclusion................ 107 


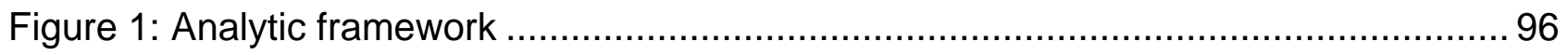

Figure 2: Flow chart of literature search and screening results.................................. 106 


\section{INTRODUCTION}

This document describes a systematic review conducted to answer the following question: What is the relationship between dietary patterns consumed during pregnancy and gestational weight gain? This systematic review was conducted by the 2020 Dietary Guidelines Advisory Committee, supported by USDA's Nutrition Evidence Systematic Review (NESR).

More information about the 2020 Dietary Guidelines Advisory Committee is available at the following website: www.DietaryGuidelines.gov.

NESR specializes in conducting food- and nutrition-related systematic reviews using a rigorous, protocol-driven methodology. More information about NESR is available at the following website: NESR.usda.gov.

NESR's systematic review methodology involves developing a protocol, searching for and selecting studies, extracting data from and assessing the risk of bias of each included study, synthesizing the evidence, developing conclusion statements, grading the evidence underlying the conclusion statements, and recommending future research. A detailed description of the systematic reviews conducted for the 2020 Dietary Guidelines Advisory Committee, including information about methodology, is available on the NESR website: https://nesr.usda.gov/2020-dietary-guidelines-advisory-committee-systematic-reviews. In addition, starting on page 95, this document describes the final protocol as it was applied in the systematic review. A description of and rationale for modifications made to the protocol are described in the 2020 Dietary Guidelines Advisory Committee Report, Part D: Chapter 2. Food, Beverage, and Nutrient Consumption During Pregnancy. 


\section{List of abbreviations}

\begin{tabular}{ll}
\hline Abbreviation & Full name \\
\hline AHEI & Alternative Healthy Eating Index \\
\hline AMDR & Acceptable Macronutrient Distribution Range \\
\hline DASH & Dietary Approaches to Stop Hypertension \\
\hline DII & Dietary Inflammatory Index \\
\hline DP & Dietary pattern \\
\hline EVOO & Extra virgin olive oil \\
\hline FFQ & Food frequency questionnaire \\
\hline GDM & Gestational diabetes mellitus \\
\hline GWG & Gestational weight gain \\
\hline HDI & Human Development Index \\
\hline HEI & Healthy Eating Index \\
\hline HFII & Healthy Food Intake Index \\
\hline HHS & Department of Health and Human Services \\
\hline IOM & Institute of Medicine \\
\hline MDA & Mediterranean Diet Adherence \\
\hline MDQS & Maternal Diet Quality Score \\
\hline MED & Mediterranean \\
\hline NESR & Nutrition Evidence Systematic Review \\
\hline NFFD & Norwegian Fit for Delivery Diet \\
\hline NND & New Nordic Diet \\
\hline PCA & Principal component analysis \\
\hline PPWL & Postpartum weight loss \\
\hline PUFA & Polyunsaturated fatty acid \\
\hline
\end{tabular}




\begin{tabular}{ll}
\hline Abbreviation & Full name \\
\hline RoB & Risk of bias \\
\hline SES & Socioeconomic status \\
\hline USDA & United States Department of Agriculture \\
\hline
\end{tabular}




\section{WHAT IS THE RELATIONSHIP BETWEEN DIETARY PATTERNS CONSUMED DURING PREGNANCY AND GESTATIONAL WEIGHT GAIN?}

\section{PLAIN LANGUAGE SUMMARY}

\section{What is the question?}

- The question is: What is the relationship between dietary patterns consumed during pregnancy and gestational weight gain?

What is the answer to the question?

- Limited evidence suggests that certain dietary patterns during pregnancy are associated with a lower risk of excessive gestational weight gain during pregnancy. These patterns are higher in vegetables, fruits, nuts, legumes, fish, and lower in added sugar, and red and processed meat.

\section{Why was this question asked?}

- This important public health question was identified by the U.S. Departments of Agriculture (USDA) and Health and Human Services (HHS) to be examined by the 2020 Dietary Guidelines Advisory Committee.

\section{How was this question answered?}

- The 2020 Dietary Guidelines Advisory Committee, Pregnancy and Lactation Subcommittee conducted a systematic review to answer this question with support from the Nutrition Evidence Systematic Review (NESR) team.

- Dietary patterns were defined as the quantities, proportions, variety, or combination of different foods, drinks, and nutrients (when available) in diets, and the frequency with which they are habitually consumed.

- Diets based on macronutrient distribution were examined when at least one macronutrient proportion was outside of the acceptable macronutrient distribution range (AMDR) for carbohydrate, fat, and/or protein, whether or not the foods/food groups consumed were provided.

What is the population of interest?

- The population of interest is generally healthy, pregnant women.

\section{What evidence was found?}

- This review includes 26 articles published between 2009 and 2019.

- Many studies showed that "beneficial" dietary patterns were related to less weight gain during pregnancy.

- Dietary patterns that were consistently related to less weight gain during pregnancy were:

○ higher in vegetables, fruits, nuts, legumes, fish, and

- lower in added sugar, red and processed meat.

- The body of evidence is limited in several ways:

- Studies were mostly observational and cause-effect relationships between diet and weight gain during pregnancy were therefore difficult to determine. 
- There was little racial/ethnic, socioeconomic, and age diversity in these studies.

- It was difficult to compare studies due to inconsistencies in how diets were measured.

- There were concerns about potential bias of the studies.

- Many studies were not designed to study the relationship between dietary patterns and weight gain during pregnancy.

\section{How up-to-date is this systematic review?}

- This review searched for studies from January 2000 to November 2019. 


\section{TECHNICAL ABSTRACT}

\section{Background}

- This important public health question was identified by the U.S. Departments of Agriculture (USDA) and Health and Human Services (HHS) to be examined by the 2020 Dietary Guidelines Advisory Committee.

- The 2020 Dietary Guidelines Advisory Committee, Pregnancy and Lactation Subcommittee conducted a systematic review to answer this question with support from the Nutrition Evidence Systematic Review (NESR) team.

- The goal of this systematic review was to examine the following question: What is the relationship between dietary patterns consumed during pregnancy and gestational weight gain?

\section{Conclusion statement and grade}

- Limited evidence suggests that certain dietary patterns during pregnancy are associated with a lower risk of excessive gestational weight gain during pregnancy. These patterns are higher in vegetables, fruits, nuts, legumes, fish, and lower in added sugar, and red and processed meat. (Grade: Limited)

\section{Methods}

- A literature search was conducted using four databases (PubMed, Cochrane, Embase, and CINAHL) to identify articles that evaluated the intervention/exposure of dietary patterns during pregnancy and the outcome of gestational weight gain. A manual search was conducted to identify articles that may not have been included in the electronic databases searched. Articles were screened by two NESR analysts independently for inclusion based on pre-determined criteria.

- Data extraction and risk of bias assessment were conducted for each included study, and both were checked for accuracy. The Committee qualitatively synthesized the body of evidence to inform development of a conclusion statement(s), and graded the strength of evidence using pre-established criteria for risk of bias, consistency, directness, precision, and generalizability.

\section{Summary of the evidence}

- This systematic review includes 26 articles, including five from four randomized controlled trials (RCTs) and 21 from 19 prospective cohort studies published between 2009 and 2019.

- Articles included in this review assessed one of the following interventions/exposures during pregnancy:

- Dietary patterns (DPs) (24 studies).

- Diets based on macronutrient distributions outside of the acceptable macronutrient distribution range (AMDR) (2 studies).

- Eight of the 15 articles that assessed maternal DPs using an index/score method showed an association with gestational weight gain (GWG).

○ Five of the eight articles showed that greater adherence to a DP (identified as beneficial by the study) was associated with lower GWG.

- Three articles showed that greater adherence to a DP (identified as 
beneficial by the study) was associated with greater GWG in all participants or only women with obesity.

- Four of the five articles that assessed maternal DPs using a factor or cluster analysis showed one or more associations between adherence to DPs and GWG.

- One article showed that greater adherence to a DP (identified as beneficial by the study) was associated with lower GWG.

- Four articles showed that greater adherence to a DP (identified as detrimental by the study) was associated with higher GWG.

- One study that assessed maternal DPs using reduced rank regression showed that greater adherence to a DP was associated with higher GWG.

- Two RCTs showed that participants randomized to a DP (identified as beneficial by the study) had lower GWG.

- One RCT and one prospective cohort study showed no association between maternal consumption of a diet higher in fat (i.e. $>35$ percent of total energy from fat, which is greater than the AMDR) and GWG.

- Although the DPs examined were characterized by combinations of different foods and beverages, the patterns that were consistently shown to be associated with lower risk of excessive GWG were: higher in vegetables, fruits, nuts, legumes, and fish and lower in added sugar and red and processed meat.

- Not all foods were part of the same DP. The evidence did not show a consistent association between grains or dairy and GWG.

- The ability to draw strong conclusions was limited by the following issues:

- There were few RCTs and thus data were primarily observational in nature, limiting the ability to determine causal effects of DPs on GWG.

- Key confounders were not consistently controlled for in most of the studies.

- Studies had risk-of-bias issues, including exposure misclassification, selfreported outcomes, and selection bias.

- Most of the studies were not designed to assess the association between DPs and GWG.

- People with lower SES, adolescents, and racially and ethnically diverse populations were underrepresented in the body of evidence. 


\section{FULL REVIEW}

\section{Systematic review question}

What is the relationship between dietary patterns consumed during pregnancy and gestational weight gain?

\section{Conclusion statement and grade}

Limited evidence suggests that certain dietary patterns during pregnancy are associated with a lower risk of excessive gestational weight gain during pregnancy. These patterns are higher in vegetables, fruits, nuts, legumes, fish, and lower in added sugar, and red and processed meat. (Grade: Limited)

\section{Summary of the evidence}

- This systematic review includes 26 articles, ${ }^{1-26}$ including five articles from four randomized controlled trials (RCTs) and 21 articles from 19 prospective cohort studies published between 2009 and 2019.

- Articles included in this review assessed one of the following interventions/exposures during pregnancy:

○ Dietary patterns (DP) (24 studies).

- Diets based on macronutrient distributions outside of the acceptable macronutrient distribution range (AMDR) (2 studies).

- Eight of the 15 articles that assessed maternal DPs using an index/score method showed an association with gestational weight gain (GWG):

- Five of the eight articles showed that greater adherence to a DP (identified as beneficial by the study) was associated with lower GWG.

- Three articles showed that greater adherence to a DP (identified as beneficial by the study) was associated with greater GWG in all participants or only women with obesity.

- Four of the five articles that assessed maternal DPs using a factor or cluster analysis showed one or more associations between adherence to DPs and GWG.

- One article showed that greater adherence to a DP (identified as beneficial by the study) was associated with lower GWG.

- Four articles showed that greater adherence to a DP (identified as detrimental by the study) was associated with higher GWG.

- One study that assessed maternal DPs using reduced rank regression showed that greater adherence to a DP was associated with higher GWG.

- Two RCTs showed that participants randomized to a DP (identified as beneficial by the study) had lower GWG

- One RCT and one prospective cohort study showed no association between maternal consumption of a diet higher in fat (i.e., $>35$ percent of total energy from fat, which is greater than the AMDR) and GWG

- Although the DPs examined were characterized by combinations of different foods and beverages, the patterns that were consistently shown to be associated with lower risk of excessive GWG were: higher in vegetables, fruits, nuts, legumes, and fish and lower in added sugar and red and processed meat.

- Not all foods were part of the same DP. The evidence did not show a 
consistent association between grains or dairy and GWG.

- The ability to draw strong conclusions was limited by the following issues:

o There were few RCTs and thus data were primarily observational in nature, limiting the ability to determine causal effects of DPs on GWG.

- Key confounders were not consistently controlled for in most of the studies.

- Studies had risk-of-bias issues, including exposure misclassification, selfreported outcomes, and selection bias.

- Most of the studies were not designed to assess the association between DPs and GWG.

- People with lower SES, adolescents, and racially and ethnically diverse populations were underrepresented in the body of evidence.

\section{Description of the evidence}

This systematic review included articles that address the relationship between dietary patterns and/or diets based on macronutrient distributions outside of the AMDR during pregnancy and gestational weight gain (GWG). The search included articles from high and very high Human Development Indexii countries and the search time frame spanned between January 2000 and November 2019. Studies considered in this body of evidence included pregnant women who were generally healthy. The following study designs were included: randomized controlled trials (RCTs), non-randomized controlled trials, prospective and retrospective cohort studies, and nested case-control studies.

This body of evidence includes 26 articles, including five from four RCTs ${ }^{22-26}$ and 21 from 19 prospective cohort studies. ${ }^{1-21}$ A total of 23 distinct trials/cohort studies are represented in this body of evidence. References are included for each study in Table 1 with trial/cohort names or locations when names were unavailable. Six studies were conducted in the U.S. ${ }^{6,7,9,11-13,16,25}$ In addition, three studies were conducted in Spain, ${ }^{1,2,23,26}$ two each in the U.K., ${ }^{22,24}$ Norway, ${ }^{5,21}$ and Japan ${ }^{3,4}$; and one each in Mexico, ${ }^{18}$ Italy, ${ }^{19}$ Poland, ${ }^{17}$ Iceland, ${ }^{14}$ Finland, ${ }^{10}$ the Netherlands, ${ }^{8}$ China, ${ }^{15}$ and Malaysia. ${ }^{20}$

\footnotetext{
ii The Human Development classification was based on the Human Development Index (HDI) ranking from the year the study intervention occurred or data were collected (UN Development Program. HDI 1990-2017 HDRO calculations based on data from UNDESA (2017a), UNESCO Institute for Statistics (2018), United Nations Statistics Division (2018b), World Bank (2018b), Barro and Lee (2016) and IMF (2018). Available from: http://hdr.undp.org/en/data). If the study did not report the year in which the intervention occurred or data were collected, the HDI classification for the year of publication was applied. HDI values are available from 1980, and then from 1990 to present. If a study was conducted prior to 1990, the HDI classification from 1990 was applied. When a country was not included in the HDI ranking, the current country classification from the World Bank was used instead (The World Bank. World Bank country and lending groups. Available from: https://datahelpdesk.worldbank.org/knowledgebase/articles/906519-world- country-and-lending-groups).
} 
Table 1. Included trials and cohorts

\section{Randomized Controlled Trials}

Effect of Simple, Targeted Diet in Pregnant Women With Metabolic 22

Risk Factors on Pregnancy Outcomes (ESTEEM) trial

St. Carlos GDM prevention study

23,26

Maternal Offspring Metabolics Family Intervention Trial (MOMFIT)

U.K. Pregnancies Better Eating and Activity Trial (UPBEAT)

25

24

\section{Prospective Cohort Studies}

Mamma \& Bambino

Osaka Maternal and Child Health Study (OMCHS)

Generation R Study

Born in Guangzhou Cohort Study (BIGCS)

Polish Mother and Child Cohort

Pregnancy Research on Inflammation, Nutrition and City

Environments: Systematic Analyses (PRINCESA)

New Hampshire Birth Cohort Study (NHBCS)

INfancia y Medio Ambiente (INMA)

Project Viva

Mérida Cohort

Norwegian Fit for Delivery (NFFD)

Norwegian Mother and Child Cohort Study (MoBa)

PREgnant Women of Iceland (PREWICE)

Control arm of RADIEL trial

Infant Feeding Practices Study II (IFPSII)

Seremben Cohort Study (SECOST)

Pregnancy Environment and Lifestyle Study (PETALS)

Healthy Start
19

\section{3}

8

15

17

18

13

1

$6,9,12$

2

21

5

14

10

7

20

16

11 


\section{Subject characteristics:}

- Sample sizes of the studies ranged from $35^{2}$ to $66,597^{5}$ participants.

- Almost all of the studies were conducted in adult women (18-45y) who had singleton pregnancies. Of note, Fulay et al ${ }^{12}$ and Wedolowska et al ${ }^{17}$ enrolled mothers as young as $15 \mathrm{y}$ and $17 \mathrm{y}$, respectively.

- Health characteristics:

- Prepregnancy BMI: Seven studies enrolled predominantly or exclusively overweight or obese women. $7,10,11,16,18,24,25$ In addition, Al Wattar et al enrolled women with metabolic risk factors, including women with obesity. 22

- Diabetes: 10 studies excluded women with a previous diagnosis of type 1 and/or 2 diabetes mellitus (as defined by the studies). $5,9-12,16,17,19,21,22,25$

Two studies excluded women with one or more of the following conditions: gestational diabetes, hypertension, and preeclampsia. ${ }^{18,19}$ In an RCT conducted in Spain, the authors noted that approximately 25 percent of the participants had a family history of type 2 diabetes mellitus. ${ }^{23,26}$

- Race/ethnicity: Thirteen of the 26 articles noted that the participants were predominantly or exclusively White (defined as $\geq 50$ percent of the participants). 2,6-9,11-13,21,23-26 Nine studies did not report race/ethnicity. ${ }^{1,3,5,10,14,15,17-19}$ Yong et $\mathrm{al}^{20}$ reported that 89 percent of the participants were Malay and Tajima et $\mathrm{al}^{4}$ noted that 100 percent of their participants were Japanese. Zhu et al ${ }^{16}$ reported that the participants were predominantly Hispanic (approximately 41.3 percent) and Al Wattar et al ${ }^{22}$ noted that the participants were predominantly Asian (approximately 43.7 percent).

- Socio-economic status:

- Maternal education:

- A majority of the studies reported that the participants had some college education. ${ }^{6-9,11-16,23,25,26}$ When studies reported the years of education, most noted that a majority of the participants had $\geq 12 \mathrm{y}$ of education..$^{3,17,20,21}$ Maugeri et $\mathrm{al}^{19}$ noted that $<20$ percent of the participants had low-medium education (defined as $\leq 8 \mathrm{y}$ of education), but did not give other details. Similarly, Gesteiro et al ${ }^{2}$ reported that 83 percent of the participants had medium or high education (but did not categorize education further).

- However, the following were exceptions: Ancira-Moreno et al noted that $>50$ percent of the participants had $<9$ y of education. ${ }^{18}$ Fernandez-Barres et $\mathrm{al}^{1}$ reported that 63.6 percent of the participants had secondary education or less.

- Four studies did not report maternal education. 4,5,22,24

(Note: Fulay et al, ${ }^{12}$ who used Project Viva data, reported that approximately 32 percent of their participants had a college education, 
whereas Rifas-Shiman et $\mathrm{al}^{6}$ and Sen et al, ${ }^{9}$ who also used Project Viva data with a similar sample size as Fulay et al, reported that approximately 69 percent had college degrees).

- Income: In the 17 articles that reported household income and/or participant employment status, the majority of women were employed and/or from middle-to-high income households. 1,3,6-9,12,15-17,19,21,23,25,26 Two studies ${ }^{20,24}$ reported that the majority of participants were from low SES backgrounds.

\section{Interventions/Exposures}

\section{Dietary Patterns}

Dietary pattern (DP) was defined as the quantities, proportions, variety, or combination of different foods, drinks, and nutrients (when available) in diets, and the frequency with which they were habitually consumed. At minimum, there had to be a description of the foods and beverages in the pattern. Dietary patterns may have been measured or derived using a variety of approaches, such as adherence to a priori patterns (indices/scores), data driven patterns (factor or cluster analysis), reduced rank regression, or other methods, including clinical trials.

Dietary patterns were assessed using 1) index/score analysis, 2) factor analysis and principal component analysis (PCA), 3) experimental diet, and 4) reduced rank regression. A description of the studies categorized by the method used to measure dietary patterns is included below:

Index/Score Analysis

Fifteen articles included in this review used one or more of the following indices/scores summarized below:

- Maternal Diet Quality Score (MDQS) ${ }^{18}$

- Alternative Healthy Eating Index (AHEI) and its modifications $s^{6,7,13}$

- Healthy Eating Index $(\mathrm{HEI})^{2,16,20}$

- relative Mediterranean Diet (rMED) score ${ }^{1}$

- Dietary Approaches to Stop Hypertension (DASH) and its modifications ${ }^{12}$

- Mediterranean Diet Adherence (MDA) ${ }^{2}$

- New Nordic Diet (NND) $)^{5}$

- Norwegian Fit for Delivery (NFFD) Diet ${ }^{21}$

- Dietary risk scores ${ }^{14}$

- Healthy Food Intake Index (HFII) ${ }^{10}$

- Dietary Inflammatory Index (DII) ${ }^{9}$

- Dutch Healthy Diet Index ${ }^{8}$

Factor analysis and PCA

Five studies included in this review assessed dietary patterns using factor analysis or PCA. ${ }^{3,8,15,17,19}$

Experimental Diet

Three RCTs ${ }^{22,23,25,26}$ in this review assigned participants to an experimental diet (i.e. Mediterranean diet, DASH diet) or a control diet. 
Reduced Rank Regression

One study ${ }^{11}$ assessed adherence to DPs (patterns 1 and 2 ) derived using reduced rank regression.

Table 2. Dietary pattern components ${ }^{\mathrm{iii}}$

\begin{tabular}{|c|c|c|}
\hline Reference & Dietary pattern & Dietary components \\
\hline \multicolumn{3}{|l|}{ Index/Score Analysis } \\
\hline \multirow[t]{2}{*}{ Ancira-Moreno et al ${ }^{18}$} & MDQS & $\begin{array}{l}\text { Vegetables and fruits; legumes; low-fat dairy } \\
\text { products; PUFAs (positive) }\end{array}$ \\
\hline & & $\begin{array}{l}\text { Red meat; added sugars; foods high in saturated } \\
\text { fat or added sugar (negative) }\end{array}$ \\
\hline \multirow[t]{3}{*}{ Emond et $\mathrm{al}^{13}$} & AHEI-2010 & $\begin{array}{l}\text { Vegetables; fruits; whole grains; nuts and } \\
\text { legumes; long-chain (n-3) fats; PUFAs (positive) }\end{array}$ \\
\hline & & $\begin{array}{l}\text { Red and processed meats; sugar-sweetened } \\
\text { beverages and fruit juice; trans fats; sodium } \\
\text { (negative) }\end{array}$ \\
\hline & & Alcohol component excluded \\
\hline \multirow[t]{3}{*}{ Fernández-Barrés et al ${ }^{1}$} & rMED & $\begin{array}{l}\text { Vegetables; fruits and nuts; cereals; legumes; fish; } \\
\text { olive oil (positive) }\end{array}$ \\
\hline & & Meat; dairy (negative) \\
\hline & & Alcohol component excluded \\
\hline \multirow[t]{4}{*}{ Fulay et al ${ }^{12}$} & DASH & $\begin{array}{l}\text { Vegetables; fruits; whole grains; nuts and } \\
\text { legumes; low-fat dairy (positive) }\end{array}$ \\
\hline & & $\begin{array}{l}\text { Red and processed meat; sugar-sweetened } \\
\text { beverages; sodium (negative) }\end{array}$ \\
\hline & DASH OMNI & $\begin{array}{l}\text { Vegetables; fruits; whole grains; nuts and } \\
\text { legumes; low-fat dairy; PUFAs; MUFAs (positive) }\end{array}$ \\
\hline & & $\begin{array}{l}\text { Red and processed meat; sugar-sweetened } \\
\text { beverages; sodium (negative) }\end{array}$ \\
\hline \multirow[t]{2}{*}{ Gesteiro et $\mathrm{al}^{2}$} & $\mathrm{HEI}$ & $\begin{array}{l}\text { Vegetables; fruits; cereals, grains, and legumes; } \\
\text { meat, eggs, and fish; milk and dairy products; } \\
\text { dietary variety }\end{array}$ \\
\hline & & Total fats; SFAs; cholesterol; sodium \\
\hline
\end{tabular}

iii EVOO: extra virgin olive oil, MUFA: monounsaturated fatty acid, OMNI: optimal macronutrient intake, PCA: principal component analysis, PUFA: polyunsaturated fatty acid, SFA: saturated fatty acid 


\section{Reference Dietary pattern Dietary components}

MDA

Total vegetables; raw vegetables; fruits (including natural fruit juices); legumes; nuts (including peanuts); chicken, turkey, or rabbit meat; fish or shellfish; olive oil; dishes seasoned with sofrito (positive)

Red meat, veal, hamburger, or sausage; butter, margarine or cream; sweet or carbonated beverages; commercial sweets or pastries (negative)

\section{Alcohol component excluded}

Hillesund et al $2014^{5} \quad$ NND

Root vegetables; cabbages; potatoes relative to rice and pasta; Nordic fruits; foods from the wild countryside (game, fish, seafood, and native berries); whole grain breads relative to refined breads; oatmeal porridge; unsweetened milk relative to fruit juice; water relative to sweetened beverages; meal frequency (positive)

Hillesund et al $2018^{21} \quad$ NFFD diet

Vegetables with dinner; fruits or vegetables as snacks; water relative to other beverages; small portion size of one or more unhealthy food items (soda, salty crisps, or chocolate); meal frequency; never eating sweets and snacks without appreciation; reading nutrition labels (positive) Sugar-rich food items; fast-foods, snacks, or other salty food; eating beyond satiety (negative)

Hrolfsdottir et al ${ }^{14}$

Dietary risk score (13 risk factors)
Low intake of vegetables and fruits; whole grains; beans, nuts, and seeds; dairy; fish; vitamin D Low dietary variety High intake of processed meat; french fries and fried potatoes; dairy; sweets, ice cream, cakes, and cookies; sugar and artificially sweetened beverages; butter relative to oil

Dietary risk score (6 Low intake of vegetables and fruits; whole grains; risk factors) dairy

Low dietary variety

High intake of dairy; sugar and artificially sweetened beverages 


\section{Reference \\ Dietary pattern Dietary components}

Dietary risk score (4 Low intake of whole grains; dairy

risk factors)

High intake of dairy; sugar and artificially sweetened beverages

Meinila et al ${ }^{10} \quad$ HFII

Vegetables; fruits and berries; high-fiber grains; fish; low-fat milk; low-fat cheese; vegetable oils as cooking fat; oil-based fat spreads (positive)

Sugar-sweetened beverages (including juice); fast food; snacks with added sugar or salt (negative)

Poon et $\mathrm{al}^{7}$

AHEI-P

Vegetables; whole fruit; whole grains; nuts and legumes; long-chain ( $\mathrm{n}-3)$ fats; PUFAs; calcium; folate; iron (positive)

Red and processed meats; sugar-sweetened beverages; trans fats; sodium (negative)

Alcohol component excluded

Rifas-Shiman et al ${ }^{6}$

AHEI-P

Vegetables (includes tofu and soybeans); fruits; ratio of white (poultry, fish) to red meat (beef, pork, lamb, processed); ratio of PUFAs to SFAs; fiber; calcium; folate; iron (positive)

Trans fats (negative)

Alcohol and nut components excluded

Sen et $\mathrm{al}^{9}$

DII

Vegetables; fruits; whole grains; fish and seafood; whole eggs (positive)

Red and processed meat; sugar-sweetened soda (negative)

Tielemans et $\mathrm{al}^{8} \quad$ Dutch Healthy Diet Index

Vegetables; fruits; fish; fiber (positive)

SFAs; sodium (negative)

Alcohol, acidic food and drink, and trans fat components excluded

Yong et $\mathrm{al}^{20}$

Modified HEI for Malaysians

Vegetables; fruits; cereals and grains; legumes; poultry, meat, and eggs; fish and seafood; milk and milk products; total fats; sodium (negative)

Zhu et al ${ }^{16}$

HEI-2010

Total vegetables; beans and greens; total fruit; whole fruit; whole grains; total protein foods; seafood and plant proteins; dairy; ratio of PUFAs and MUFAs to SFAs (positive) 


\section{Reference Dietary pattern Dietary components}

Refined grains; calories from solid fats and added sugars; sodium (negative)

Alcohol component excluded

\section{Factor Analysis and PCA ${ }^{\text {iv }}$}

\begin{tabular}{|c|c|c|}
\hline \multirow[t]{2}{*}{ Maugeri et al ${ }^{19}$} & Western & $\begin{array}{l}\text { High intake of red meat, fries, dipping sauces, } \\
\text { salty snacks and alcoholic drinks }\end{array}$ \\
\hline & Prudent & $\begin{array}{l}\text { High intake of boiled potatoes, cooked vegetables, } \\
\text { legumes, pizza and soup }\end{array}$ \\
\hline \multirow[t]{3}{*}{ Okubo et $\mathrm{al}^{3}$} & Meat and eggs & $\begin{array}{l}\text { High intake of beef and pork, processed meat, } \\
\text { chicken, eggs, butter, and dairy products }\end{array}$ \\
\hline & Wheat products & $\begin{array}{l}\text { High intake of bread, confectioneries, fruit and } \\
\text { vegetable juice, and soft drinks }\end{array}$ \\
\hline & $\begin{array}{l}\text { Rice, fish, and } \\
\text { vegetables }\end{array}$ & $\begin{array}{l}\text { High intake of rice, potatoes, nuts, pulses, fruits, } \\
\text { green and yellow vegetables, white vegetables, } \\
\text { mushrooms, seaweeds, Japanese and Chinese } \\
\text { tea, fish, shellfish, sea products, miso soup, and } \\
\text { salt-containing seasoning }\end{array}$ \\
\hline \multirow[t]{3}{*}{ Tielemans et $\mathrm{al}^{8}$} & $\begin{array}{l}\text { Vegetable, Oil and } \\
\text { Fish }\end{array}$ & High intake of vegetables, oil, and fish \\
\hline & $\begin{array}{l}\text { Nuts, High-Fiber } \\
\text { Cereals, and Soy }\end{array}$ & High intake of nuts, high-fiber cereals, and soy \\
\hline & $\begin{array}{l}\text { Margarine, Sugar, } \\
\text { and Snacks }\end{array}$ & High intake of margarine, sugar, and snacks \\
\hline \multirow[t]{6}{*}{ Wei et al ${ }^{15}$} & Cereals & Richer in cereals \\
\hline & Vegetables & Richer in vegetables \\
\hline & Meats & Richer in meats \\
\hline & Fruits & Richer in fruits \\
\hline & $\begin{array}{l}\text { Fish, beans, nuts, } \\
\text { and yogurt }\end{array}$ & Richer in fish, beans, nuts, and yogurt \\
\hline & $\begin{array}{l}\text { Milk and milk } \\
\text { powder }\end{array}$ & Richer in milk and milk powder \\
\hline
\end{tabular}

${ }^{\text {iv }}$ Author-derived dietary pattern 


\section{$\begin{array}{lll}\text { Reference Dietary pattern Dietary components } & \text { D }\end{array}$}

Wesolowska et al ${ }^{17}$

Western

High intake of refined grains, processed meat, potatoes, and very low intake of whole grains

Mixed Intake between Western and Prudent

Prudent

High intake of fruit, vegetables, legumes, whole grains, poultry, and low-fat dairy products

\section{Experimental Diet}

Al Wattar et al ${ }^{22} \quad$ MED-style Diet

High consumption of nuts, extra virgin olive oil, fruits, vegetables, non-refined grains, and legumes; moderate-to-high consumption of fish; low-to-moderate amounts of poultry and dairy products, such as yogurt and cheese; low consumption of red meat and processed meat; avoidance of sugary drinks, fast food, and foods rich in animal fat. Mixed nuts (walnuts, hazelnuts, and almonds) and EVOO provided by investigators.

Control

Diet recommended as per U.K. national recommendations for antenatal care

Assaf-Balut et al ${ }^{23,26} \quad$ Control

Mediterranean Diet: High consumption of vegetables, fruit (avoiding juices), skimmed dairy products, whole grain cereals, and legumes; moderate-to-high consumption of fish; low consumption of red and processed meat; avoidance of refined grains, processed baked goods, pre-sliced bread, soft drinks and fresh juices, fast foods, and precooked meals; restriction of dietary fat, including EVOO and nuts

Intervention

Mediterranean Diet plus EVOO and pistachios provided by investigators

Encouraged consumption of low-fat dairy products, fish, skinless poultry, lean meat and vegetable protein, unsaturated fats, fiber-rich whole grains, fruits, vegetables, and legumes; discouraged consumption of sugar-sweetened beverages, sweets, and non-nutrient-dense snack foods; avoidance of fish considered higher in mercury; inclusion of calcium-rich, vitamin D-enriched dairy, or calcium-fortified non-dairy products 


\section{Reference \\ Dietary pattern \\ Dietary components}

Control

Usual care, including bi-weekly newsletters and publicly available maternity website links

\section{Reduced Rank Regression ${ }^{v}$}

Starling et al ${ }^{11}$

Pattern 1

Higher consumption of poultry, nuts, cheese, fruits, whole grains, added sugars, and solid fats

Pattern 2

Higher consumption of eggs, starchy vegetables, solid fats, fruits, and non-whole grains and a lower consumption of dairy foods, dark-green vegetables, and whole grains

${ }^{v}$ Author-derived dietary pattern 


\section{Diets based on Macronutrient Distribution}

Studies assessing diets based on macronutrient distributions outside of the acceptable macronutrient distribution range (AMDR) had to specify the distribution of carbohydrate, fat, and protein in the diet to be considered for this review. Macronutrient proportions outside of the AMDR are as follows:

- Carbohydrate for all age groups: $<45$ or $>65$ percent of energy;

- Protein for $\geq 19$ years: $<10$ or $>35$ percent of energy;

- Fat for $\geq 19$ years: $<20$ or $>35$ percent of energy

One RCT and one prospective cohort study 4,24 in this review assessed macronutrient proportions and reported the percentage of total energy from fat was higher than the AMDR for one of the groups.

\section{Time point of exposure}

The RCTs ${ }^{22-26}$ randomized women during the late first trimester ${ }^{23,26}$ or early second trimester ${ }^{22,24,25}$ and followed them to the end of the second trimester. ${ }^{22-26}$

Most of the cohort studies administered food frequency questionnaires (FFQ) or 24 hour recalls toward the end of the first trimester or early in the second. ${ }^{2,6,8-10,12,14,16,19-21}$ Investigators typically collected retrospective dietary data reflecting early pregnancy intake (i.e. first trimester). In a few studies, maternal dietary data were collected in the second trimester ${ }^{3,5,13,15,17}$ or third trimester, ${ }^{1,7}$ reflecting intake in the past week, ${ }^{15}$ month, ${ }^{3,7}$ or the entire pregnancy until that point. ${ }^{13,17}$ In a few cohorts, dietary data were collected at multiple time points and assessed at individual time points (e.g. by trimester) or combined in the analysis. ${ }^{6,9,11,20}$

\section{Outcome}

The outcome considered in this review was gestational weight gain (GWG), reported in a number of ways, including total GWG, 2,3,7,11,12,15,16,19,22,25 GWG for a specified time period ${ }^{23,24,26}$ or trimester, ${ }^{10}$ GWG rate, $1,4,8,15,18,20$ and GWG adequacy. . $^{5,6,8,9,13-15,17-}$ $21,23,25$ The studies that assessed GWG adequacy classified GWG as inadequate, adequate, or excessive according to the Institute of Medicine (2009) guidelines ${ }^{\mathrm{vi}}$, with the exception of Rifas-Shiman et al, ${ }^{6}$ who used Institute of Medicine (1990) guidelines vii, Hrolfsdottir et al, ${ }^{14}$ who used Icelandic recommendations (no reference provided), and Assaf-Balut et al, ${ }^{23}$ who had cited a previous publication viii.

\footnotetext{
vi Institute of Medicine (US) and National Research Council (US) Committee to Reexamine IOM Pregnancy Weight Guidelines. Weight Gain During Pregnancy: Reexamining the Guidelines. (Rasmussen KM, Yaktine AL, eds.). Washington (DC): National Academies Press (US); 2009

vii Institute of Medicine (US) Committee on Nutritional Status During Pregnancy and Lactation. Nutrition During Pregnancy: Part I Weight Gain. Washington (DC): National Academies Press (US); 1990

viii Hutcheon JA, Platt RW, Abrams B, Himes KP, Simhan HN, Bodnar LM. Pregnancy weight gain charts for obese and overweight women. Obesity. 2015; 23(3): 532-535. doi: 10.1002/oby.21011.
} 


\section{Evidence synthesis}

With 26 articles, there is a substantial body of evidence available to examine the association between DPs during pregnancy and GWG (Table 3, Table 4). However, there is heterogeneity in the methods used to define and assess DPs and how outcomes were reported, which made it difficult to compare results across studies. Furthermore, the time period of dietary assessment and the duration of the recall also varied across studies.

\section{Dietary patterns assessed via index/score}

Fifteen articles used indices/scores to assess DPs and the findings are described below:

- Ancira-Moreno et al ${ }^{18}$ assessed the association between adherence to the Maternal Diet Quality Score (MDQS) and GWG trajectories (baseline $\mathrm{n}=660$ ) in Mexican participants. The MDQS was based on the Mexican Dietary Guidelines and international recommendations for specific foods and was characterized by higher intake of PUFAs, fruits and vegetables, legumes, and low-fat dairy products; lower in foods higher in saturated fat, added sugars, and red meat. The study accounted for key confounders including age, SES, physical activity, prepregnancy BMI, GDM, hypertension, and parity. Anciro-Moreno et al also adjusted for energy intake (kilocalories per day) in the model when assessing whether diet quality was independent of total energy. The study noted the following findings:

- Medium and high adherence throughout pregnancy were associated with lower risk of inadequate weight gain, but only high adherence was associated with lower risk of excessive weight gain.

- When the investigators assessed specific time periods during pregnancy they noted that:

- Medium adherence to the MDQS was associated with significantly lower rate of weight gain during middle pregnancy (20-30 weeks).

- In late pregnancy (30-40 weeks), medium and higher adherence were positively associated with the rate of GWG.

- When looking at weight gain beyond 40 weeks (defined by authors as prolonged pregnancy), higher adherence was associated with lower rate of weight gain.

- Emond et $\mathrm{al}^{13}$ examined maternal adherence to the Alternative Healthy Eating Index-2010 (AHEI) and GWG in a U.S.-based cohort (baseline $\mathrm{n}=1,140$ ). The AHEI-2010 was characterized by higher intake of six 'healthful' components, including: fruits, vegetables, whole grains, nuts and legumes, long-chain n-3 fatty acids from foods and supplements, and PUFAs; and lower intake of four components, including: sugar-sweetened beverages and fruit juice, red and processed meats, trans fatty acids, and sodium. The primary objective of this study was to assess the association between the AHEI-2010 and infant outcomes. The study reported that there were no significant differences in GWG adequacy (i.e., insufficient, adequate, excessive) based on different levels of 
adherence to the AHEI-2010. None of the key confounders were accounted for in the analysis.

- In a Spain-based cohort study with a baseline sample size of 2,195 participants, Fernandez-Barres et al ${ }^{1}$ assessed maternal adherence to the relative Mediterranean Diet ( $M E D$ ) score, which was constructed by taking into account the consumption of vegetables, fruits and nuts, cereals, legumes, fish, olive oil, meat, and dairy products. The scoring for meat and dairy products was reversed. Although GWG was assessed, the study was designed to examine the association between the rMED and the child's longitudinal BMI and cardiometabolic risk. The study reported that highest adherence to the rMED DP was associated with significantly lower mean GWG (kilogram per week). The study did not account for any of the key confounders.

- In the U.S.-based Project Viva study (baseline $n=2,128$ ), Fulay et $\mathrm{al}^{12}$ examined the association between the DASH diet and GWG, in addition to other pregnancy outcomes. The authors noted that the DASH diet was similar to the Mediterranean diet in that both are rich in fruits, vegetables, legumes, whole grains, and healthy fats, with limited amounts of poultry, red meat, and dairy. In addition, the DASH diet emphasized reduced intake of sodium, saturated fat, total fat, and cholesterol, and higher intake of fiber and protein. As a variation of DASH, the authors also assessed the association between the DASH OMNI (supplemented by higher unsaturated fat intake) and GWG. After adjusting for total energy intake and key confounders (including age, race/ethnicity, SES, prepregnancy BMI, smoking and parity) in a statistical model, the authors reported that greater adherence to the DASH and DASH OMNI diets was associated with greater subsequent GWG. This was primarily observed among women who were obese during prepregnancy $\left(\geq 30 \mathrm{~kg} / \mathrm{m}^{2}\right)$ and not in those who were overweight, normal weight or underweight.

- Gesteiro et $a^{2}$ examined the association between maternal Mediterranean Diet Adherence (MDA), the Healthy Eating Index (HEI), and GWG, in a Spanish cohort study (baseline $\mathrm{n}=35$ ).

- High MDA ( $\geq 7)$ was characterized by the use of olive oil as the main dietary fat, higher intakes of vegetables, raw vegetables, fruits, fish or shellfish, nuts (including peanuts), legumes, chicken, turkey or rabbit meat, and dishes seasoned with sofrito, sauce made with tomato, onion, leek or garlic, and simmered with olive oil, and lower intakes of red meat, veal, pork, hamburger or sausage, butter, margarine or cream, sweet or carbonated beverages, and commercial sweets or pastries.

- The HEI was characterized by intake of cereals, grains and legumes, vegetables, fruits, milk and dairy products, meat, eggs and fish, total fat, saturated fat, cholesterol (milligrams per day), and sodium (grams per day). HEl also accounted for the intake of total fat, saturated fat, sodium and dietary variety.

The primary objective of this study was to assess the association between first trimester diet quality, measured by the HEI and MDA, and insulin sensitivity/resistance biomarkers at birth. The study accounted for key 
confounders, including race/ethnicity, smoking, and GDM. The investigators noted that women with higher MDA ( $\geq 7)$ had significantly higher total GWG, when compared to those with lower MDA $(<7)$. There were no significant differences in GWG between women with adequate vs. inadequate $\mathrm{HEI}$ adherence.

- Hillesund et al ${ }^{5}$ examined the association between the New Nordic Diet (NND) and GWG and fetal growth in this Norwegian cohort study (baseline $n=66,597$ ). The NND measured the frequency of eating of the following foods: Nordic fruits (apples, pears, plums, strawberries), root vegetables (carrots, rutabaga and various types of onions), cabbages (kale, cauliflower, broccoli and Brussels sprouts), potatoes, whole grain breads, oatmeal porridge, foods from the wild countryside (wild fish, seafood, game and wild berries), milk, and water. There were significant differences in GWG adequacy between groups, depending on the extent of NND adherence. Specifically, the proportion of women with excessive GWG was higher in the low adherence group compared to the medium or high adherence groups. When stratified by prepregnancy $\mathrm{BMI}$, these results were consistent for women with a healthy prepregnancy $\mathrm{BMI}(\mathrm{BMI}<25.0$ $\mathrm{kg} / \mathrm{m}^{2}$ ), and marginally significant for those who were overweight or obese (BMI $\left.\geq 25 \mathrm{~kg} / \mathrm{m}^{2}\right)$ prior to pregnancy $(p=0.076)$. None of the key confounders were adjusted for in the analysis.

- Hillesund et a ${ }^{21}$ assessed maternal adherence to the Norwegian Fit for Delivery (NFFD) Diet in this Norwegian cohort study (based on the Norwegian Fit for Delivery trial) with a baseline sample size of 606 . The NFFD diet was characterized by $\geq 24$ main meals per week, water for $\geq 44$ percent of drinking events, vegetables with dinner $\geq 5$ per week, fruits or vegetables as snacks $\geq 3$ per week, $<1$ per day sugar-rich food items, $<1$ per day fast-foods, snacks, or other salty food, never eating sweets and snacks without appreciation, buying small portion size of $\geq 1$ unhealthy food items, eating beyond satiety $<1$ per week, and reading nutrition labels on foods sometimes or often. The main objective of the study was to assess the association between the NFFD diet and a number of maternal and neonatal outcomes, including GWG. After accounting for the key confounders, including age, SES, prepregnancy BMI, smoking and parity, there was a statistically significant inverse association between early pregnancy NFFD diet score and the odds of excessive GWG. This association remained significant even after adjusting for physical activity, in addition to other key confounders. There was no association between diet scores and inadequate GWG.

- Hrolsdottir et al, ${ }^{14}$ an Icelandic cohort study (baseline $n=1,326$ ), assessed the association between dietary risk scores and excessive GWG. A high dietary risk score was characterized by a non-varied diet, non-adequate frequency of consumption of fruits/vegetables, dairy, and whole grain intake, and excessive intake of sugar/artificially sweetened beverages and dairy. After accounting for the key confounders, including age, SES, prepregnancy BMI, smoking, parity and GDM (only in a sub-analysis), the study showed that a higher dietary risk score (which included 6 dietary risk factors - a non-varied diet, vegetables and fruits $<5$ times per day, dairy intake $<2$ times per day, whole grain products $<2$ times per day, sugar- and artificially sweetened beverages $\geq 5$ times per week, 
dairy intake $\geq 5$ times per day) was associated with excessive GWG. Similarly higher dietary risk score with three foods (sugar- and artificially sweetened beverages $\geq 5$ times per week, whole grain products $<2$ times per day and dairy intake $\geq 5$ times per day) was associated with the risk of excessive GWG; however, there was no association between dietary risk score when 13 foods were considered (not eating a varied diet, vegetables and fruits $<5$ times per day, fish intake $<2$ times per day, dairy intake $<2$ times per day, whole grain products $<2$ times per day, beans, nuts, seeds $<3.5$ times per week, D-vitamin $<5$ times per week, quality of fat - using butter rather than oil $(\geq 50 \%)$ French fries and fried potatoes $\geq 1$ times per week, sweets, ice cream, cakes, cookies $\geq$ 2.5 times per week, sugar- and artificially sweetened beverages $\geq 5$ times per week, dairy intake $\geq 5$ times per day, processed meat products $\geq 1$ times per week).

- Meinila et al, ${ }^{10}$ a Finnish prospective cohort study (based on the control arm of the RADIEL trial), assessed the association between the Healthy Food Intake Index (HFII) and GWG (baseline $\mathrm{n}=137$ ). The primary objective of this study, however, was to assess the association between the HFII and GDM. A higher HFIl score was indicative of higher adherence to the Nordic Nutrition Recommendations (NNR), characterized by the following: 1) increased consumption of vegetables, fruits, fish and seafood, nuts and seeds; 2) substituting whole grains for refined grains, vegetable oils for butter, oil-based fat instead of butter-based spread and low-fat dairy instead of full-fat dairy; and 3) limiting beverages and foods with added sugar or salt, including snacks, sugarsweetened drinks/juice, fast foods, and red and processed meat. Adherence to the HFIl was not associated with GWG from the first to the second trimester. None of the key confounders were accounted for in the analysis.

- Poon et $\mathrm{al}^{7}$ assessed adherence to the Alternate Healthy Eating Index for Pregnancy (AHEI-P) and its association with GWG in a U.S.-based cohort study (baseline $n=893$ ). The primary objective of this study was to examine the impact of the maternal DP on the incidence of SGA and LGA. The AHEI-P was characterized by higher intake of vegetables, whole fruit, whole grains, nuts and legumes, long-chain (n-3) fatty acids, PUFAs, folate, calcium, and iron, and lower intake of sugar-sweetened beverages, red and processed meat, trans fat, and sodium. The authors reported no significant difference in GWG across different levels of adherence to the AHEI-P. None of the key confounders were accounted for in the analysis.

- Rifas-Shiman et al ${ }^{6}$ also used a modified AHEI called $A H E I-P$, characterized by the intake of vegetables (including tofu or soybean), fruit, ratio of white-to-red meat, fiber, trans fat, ratio of polyunsaturated-to-saturated fatty acids, and folate, calcium, and iron from foods. The authors made some changes to the AHEI by excluding alcohol and nuts and including folate, calcium and iron intake.

Although the AHEI-P indices assessed by Poon et $\mathrm{al}^{7}$ and Rifas-Shiman et al ${ }^{6}$ are similar, they are not the same. The main objective of this U.S.-based study (baseline $n=1,777$ ), which used Project Viva data, was to assess the maternal characteristics associated with the AHEI-P score, with GWG measured as a secondary outcome. After adjusting for the key confounders, including age, race/ethnicity, SES and prepregnancy BMI, the study found that greater 
adherence to the AHEI-P was not associated with altered risk for inadequate or excessive GWG.

- Sen et $\mathrm{al}^{9}$ also used Project Viva data to assess the association between prenatal Dietary Inflammatory Index (DII) and a number of perinatal outcomes, including GWG (baseline $n=1,808$ ). Lower DII is characterized by higher intakes of vegetables, fruit, whole-grain foods, fish/seafood, and whole eggs, and lower intakes of red or processed meats and sugar-sweetened soda. After accounting for the key confounders, including age, race/ethnicity, SES, prepregnancy BMI, smoking and parity, the study found no association between consuming a lower DII diet and GWG.

- Tielemans et $\mathrm{al}^{8}$ used the Netherlands-based Generation R study data to assess the association between an a priori and an a posteriori DP and GWG (baseline $\mathrm{n}=4,097)$. The latter findings are discussed in the next section. The Dutch Healthy Diet Index (a priori DP) consisted of 6 components, including vegetables, fruit, dietary fiber, fish, saturated fatty acids, and sodium. After accounting for the key confounders, including, age, race/ethnicity, SES, prepregnancy BMI, smoking, GDM, hypertension and parity, the study found no significant association between the Dutch Health Diet Index and GWG.

- Yong et $\mathrm{al}^{20}$ examined the association between diet quality and GWG in a Malaysia-based Seremben Cohort Study (baseline $n=480$ ). Specifically, the study used a modified HEI for Malaysians, which measured adherence to the seven food groups, including 1) cereals and grains, 2) vegetables, 3) fruits, 4) milk and milk products, 5) poultry, meat and egg, 6) fish and seafood, and 7) legumes. After adjusting for the key confounders, including, age, SES, physical activity, prepregnancy BMI and parity, the authors reported that a higher HEI score in the third trimester was associated with excessive GWG, irrespective of prepregnancy BMI. However, during the second trimester, the association between the $\mathrm{HEI}$ and GWG varied based on prepregnancy BMI. In women with a prepregnancy BMI (18.50-24.99 kg/m²), higher HEl was associated with lower odds of inadequate GWG. In women who were overweight or obese during prepregnancy, higher $\mathrm{HEl}$ was associated with increased odds of excessive GWG.

- Zhu et al, ${ }^{16}$ using the U.S.-based PETALS cohort, investigated the association between the $\mathrm{HEl}-2010$ and GWG, although the primary objective of this study was to investigate whether maternal diet quality affected fetal growth (baseline $\mathrm{n}=2,269)$. The HEl-2010 included 12 components: total fruit, whole fruit, total vegetables, greens and beans, whole grains, dairy, total protein foods, seafood and plant proteins, fatty acids, refined grains, sodium, and empty calories from solid fats, alcohol and added sugars. However, for this study, alcohol intake was excluded from the empty calories component. The study reported that total GWG did not differ among women across different levels of adherence to the HEI2010. None of the key confounders were accounted for in the analysis.

Summary: Of the 15 articles that assessed maternal DPs using an index/score method, eight showed an association with GWG. 1,2,5,12,14,18,20,21 Four of the eight showed that greater adherence to a beneficial DP was either associated with a: 1) lower risk of excessive GWG, ${ }^{5,21}$ 2) lower rate of $\mathrm{GWG},{ }^{18}$ or 3) lower mean $\mathrm{GWG} .{ }^{1} \mathrm{An}$ 
additional study showed that greater adherence to a 'detrimental' DP was associated with excessive GWG. ${ }^{14}$ However, three studies showed that higher adherence to a beneficial DP (i.e. DASH, DASH OMNI, Mediterranean Diet, HEI) was associated with higher GWG, either in all participants ${ }^{2}$ or only in obese women. ${ }^{12,20}$ Six of the eight articles $^{2,12,14,18,20,21}$ that showed an association adjusted for one or more of the key confounders.

Among the seven articles that did not show an association, ${ }^{6-10,13,16}$ four did not adjust for any of the key confounders, nor were they primarily designed to address the association between DP and GWG. $7,10,13,16$ In two of these, the timing of exposure assessment was also different, with one assessing maternal diet at the end of the second trimester ${ }^{13}$ and the other during the third trimester. ${ }^{7}$ Two other articles that did not show an association were both conducted with the same cohort (Project Viva). ${ }^{6,9}$

\section{Dietary patterns assessed via factor or principal components analysis}

Five studies 3,8,15,17,19 used data-driven methods (i.e. PCA, exploratory factor analysis, and cluster analysis) to assess dietary patterns:

- Maugeri et a $\mathrm{l}^{19}$ used PCA to generate the following dietary patterns:

- Western pattern characterized by high intake of red meat, fries, dipping sauces, salty snacks, and alcoholic drinks

- Prudent pattern characterized by high intake of boiled potatoes, cooked vegetables, legumes, pizza, and soup

The primary objective of this Italian study (baseline $n=232$ ) was to assess the association between maternal DPs and total GWG. In a statistical model, the analysis adjusted for total energy intake and key confounders, including, age, SES, smoking, GDM, hypertension, and parity. The study reported a positive trend of GWG across tertiles of a Western DP and this was more prominent in women who were obese prior to pregnancy. On the other hand, adherence to a prudent DP was positively associated with GWG among women who were underweight before pregnancy and negatively associated with GWG among women who were overweight and obese women before pregnancy.

- In a Japanese prospective cohort study (baseline $n=803$ ), Okubo et $\mathrm{al}^{3}$ used a cluster analysis to create the following dietary patterns:

- Meat and eggs pattern characterized by high intake of beef and pork, processed meat, chicken, eggs, butter, and dairy products

- Wheat products pattern characterized by high intake of bread, confectioneries, fruit and vegetable juice, and soft drinks

- Rice, fish, and vegetables pattern characterized by high intake of rice, potatoes, nuts, pulses, fruits, green and yellow vegetables, white vegetables, mushrooms, seaweeds, Japanese and Chinese tea, fish, shellfish, sea products, miso soup, and salt-containing seasoning

The primary objective of the study was to assess the association between maternal diet and neonatal anthropometric measurements at birth. The study noted that greater adherence to the wheat products DP was associated with significantly higher mean GWG when compared to the rice, fish, and vegetable DP. None of the key 
confounders were adjusted for in the analysis.

- In a Dutch cohort study with a baseline $n=4,097$, Tielemans et al ${ }^{8}$ used a PCA to generate the DPs described below:

- Vegetable, Oil, and Fish pattern characterized by higher intake of vegetables, high fat dairy products, cereals (both low and high fiber), fish and shellfish, eggs and egg products, vegetable oils, coffee and tea, alcoholic beverages, and legumes

- Nuts, High-Fiber Cereals, and Soy pattern characterized by higher intake of potatoes and other tubers, fruits, high and low fat dairy products, high fiber cereals, meat and meat products, fish and shellfish, coffee and tea, sugar-containing beverages, light soft drinks, nuts, seeds and olives, and soy products

- Margarine, Sugar, and Snacks pattern characterized by higher intake of potatoes and other tubers, high fat dairy products, low and high fiber cereals, meat and meat products, margarine and butter, sugar and confectionary and cakes, snacks, sugar-containing beverages, condiments and sauces, nuts, seeds and olives

The primary objective of the study was to investigate the association between DPs and GWG. After accounting for the key confounders, including age, race/ethnicity, SES, prepregnancy BMI, smoking and parity, none of the DPs were associated with rate of GWG (grams per week) (mid- and late-pregnancy) in normal weight or overweight women. The findings were consistent after adjusting for total energy intake in a sensitivity analysis. Similarly, none of the DPs were associated with inadequate GWG. However, greater adherence to the margarine, sugar, and snacks DP was associated with increased odds of excessive GWG compared to the lowest adherence, although per standard deviation (SD) increase in DP score was not statistically significantly associated with GWG. Tielemans et $\mathrm{al}^{8}$ also assessed the association between the Dutch Healthy Diet Index and GWG and the results were discussed in the previous section.

- In a Chinese prospective cohort study (baseline $n=5,733$ ), Wei et al $^{15}$ used cluster analysis to generate the following DPs according to the food groups that were predominant in each cluster:

- Richer in cereals characterized by higher intake of cereals (rice, pasta, porridge) and eggs (fresh and preserved)

- Richer in vegetables characterized by higher intake of leafy and cruciferous vegetables (dark green leafy vegetables, white leafy vegetables, broccoli, cauliflower)

- Richer in meats characterized by higher intake of meats (red meat, including pork, beef, and mutton, and processed meat)

- Richer in fruits characterized by higher intake of fruits (cherry/grapefruit/plum/apple/pear/peach, banana/oranges/grape, Watermelon/pineapple/mango/litchi/longan/durian and others) and Cantonese desserts 
- Richer in fish, beans, nuts, and yogurt characterized by higher intake of cereals (noodles, bread), poultry, animal organ meat (animal liver, other animal innards, animal brain and animal blood), fish (freshwater fish, seawater fish, other seafood including prawn/crab, shell fish/squid, others), bean products (soybean, other dry beans, soybean milk and bean curd), nuts (oil nuts, starchy nuts, melon), vegetables (including pumpkin/tomato/capsicums/eggplant, root vegetables including carrot, potatoes/radishes/lotus root, bean vegetables, mushrooms, sea vegetables and processed vegetables), and yogurt (snack including biscuit, cornmeal, cake/fried dough twist, confectionaries including honey, candy/chocolate, other confectionaries, sweet beverage and puffed food)

- Richer in milk and milk powder characterized by higher intake of fresh milk, pasteurized milk, formula milk powder, fat free milk powder, whole milk powder, and others

The primary objective of this Chinese study was to assess the association between maternal DPs and GWG. The study accounted for some of the key confounders, including age, SES, prepregnancy BMI and parity. Among Chinese pregnant women with a healthy prepregnancy BMI, a DP richer in fruits (compared to a DP richer in cereals) was positively associated with total GWG and GWG rate. A DP richer in fruits was also associated with an increased risk for excessive GWG. A DP richer in fish, beans, nuts and yogurt, compared to a DP richer in cereals, was positively associated with GWG rate in the second trimester and was associated with a reduced risk for inadequate GWG.

- In a Polish prospective cohort study (baseline $n=1,306$ ), Wesolowska et al, ${ }^{17}$ used an exploratory factor analysis to generate three DPs:

- Western pattern characterized by higher intake of refined grains, processed meat, potatoes, and low intake of whole grains

- Mixed pattern characterized by intakes in between Western and Prudent patterns

- Prudent pattern characterized by high consumption of fruits, vegetables, legumes, whole grains, poultry, and both low-fat and high-fat dairy products

The primary objective of the study was to evaluate sociodemographic, lifestyle, environmental, and pregnancy-related determinants of DPs during pregnancy. The study reported no significant differences between the proportions of participants that gained recommended vs. low vs. high GWG across the DPs. None of the key confounders were adjusted for in the analysis.

Summary: Of the five studies that assessed maternal DPs using factor analysis or PCA, four ${ }^{3,8,15,19}$ showed significant associations between a maternal DP and GWG. One study showed that greater adherence to a beneficial DP was associated with lower GWG, ${ }^{19}$ while the same study and three others showed that greater adherence to a non-beneficial DP was associated with higher GWG. ${ }^{3,8,15,19}$ Three of the four studies that showed an association adjusted for some of the key confounders. $8,15,19$ The only study ${ }^{17}$ that showed no association between DP and GWG did not adjust for 
any of the key confounders.

\section{Dietary patterns assessed using an experimental diet in an RCT}

Four articles from three RCTs assessed the relationship between an intervention and GWG:

- Al Wattar et al,22 in a multicenter U.K.-based trial (baseline $n=1,252$ ), randomized pregnant women with metabolic risk factors such as obesity, chronic hypertension, or hypertriglyceridemia to a Mediterranean-style diet or a control diet.

- Those randomized to the Mediterranean-style diet were encouraged to consume higher amounts of nuts, extra virgin olive oil, fruits, vegetables, nonrefined grains, and legumes; moderate-to-high amounts of fish; low-tomoderate amounts of poultry and dairy products, such as yogurt and cheese; low amounts of red meat and processed meat; and to avoid sugary drinks, fast food, and foods rich in animal fat. Investigators provided mixed nuts (30 grams per day walnuts, hazelnuts, and almonds) and EVOO (0.5 liter per week) to the participants.

- The control group received usual care and antenatal advice as per U.K. national recommendations for antenatal care.

The primary outcomes were: 1) a composite maternal outcome that combined GDM and preeclampsia, and 2) a composite infant outcome that combined stillbirth and SGA or admission to neonatal care unit. GWG was considered a secondary maternal outcome. The study showed that metabolically at-risk women randomized to a Mediterranean-style dietary pattern had lower GWG when compared to the control group.

- In a single center, clinic-based RCT in Spain (baseline $n=1,000$ ), Assaf-Balut et $\mathrm{al}^{23,26}$ randomized pregnant women to one of the following groups:

- The control group was advised to follow a Mediterranean Diet with $\geq 2$ servings vegetables per day, $\geq 3$ servings of fruits per day (avoiding juices), 3 servings skimmed dairy products per day, 3 servings wholegrain cereals per day, and 2-3 servings of legumes per week, moderate-to-high consumption of fish, low consumption of red and processed meat, avoidance of refined grains, processed baked goods, pre-sliced bread, soft drinks and fresh juices, fast foods, and precooked meals. However, they were advised to restrict the consumption of dietary fat, including EVOO and nuts.

- The intervention group was advised to follow the same Mediterranean Diet as the control group, and were advised to also consume $40 \mathrm{~mL}$ per day of EVOO and 25-30 grams per day pistachios. Investigators provided 10 liters of EVOO and 2 kilograms of roasted pistachios to the participants.

The primary outcome of this $\mathrm{RCT}^{26}$ was to assess the effect of the intervention diet on GDM incidence, with GWG as a secondary outcome. When compared to the control group, the intervention DP resulted in lower GWG between 12 to 2428 weeks gestation in women with a prepregnancy $\mathrm{BMI}<25 \mathrm{~kg} / \mathrm{m}^{2}(p=0.052)$ 
and overweight women (BMI $\geq 25$ to $\left.\leq 29.9 \mathrm{~kg} / \mathrm{m}^{2}\right)$ ( $\mathrm{p}=0.076$ ). These findings were marginally significant. However, no significant differences in GWG between the intervention and control groups were noted in women who were obese prior to pregnancy $\left(\geq 30 \mathrm{~kg} / \mathrm{m}^{2}\right)$.

When considering GWG from 12 to 36-38 weeks, the total GWG was not different between the intervention and control groups. Although non-significant, the directionality of the findings changed. In all women, the intervention group gained more total GWG than the control group between the beginning of the second trimester to the end of the third trimester $(p=0.12)$. Among women with a prepregnancy $\mathrm{BMI}<25 \mathrm{~kg} / \mathrm{m}^{2}$, the intervention group tended to gain more weight than the control group ( $p=0.096)$, whereas among obese women there was no significant difference between groups ( $\mathrm{p}=0.77)$, and among overweight women, those assigned to the control group tended to gain more weight than the intervention group $(\mathrm{p}=0.066)$.

In a sub-analysis of the St. Carlos GDM Prevention Study ${ }^{23}$ with normoglycemic women, there were no significant differences in GWG from 12 weeks to 36-38 weeks between the control and intervention groups. Also, the study did not report significant differences in weight gain between the intervention and control groups after stratifying by adequacy of weight gain (i.e. excessive, adequate, inadequate).

- In a U.S.-based RCT (baseline $\mathrm{n}=281$ ), Van Horn et $\mathrm{al}^{25}$ randomized women who were overweight or obese (prepregnancy BMI $25-40 \mathrm{~kg} / \mathrm{m}^{2}$ ) to a DASH diet and lifestyle intervention or a usual-care group, as described below:

- The intervention group was assigned to a slightly modified DASH diet (called Mama-DASH) and women were encouraged to consume a diet with low-fat dairy products, fish, skinless poultry, lean meat and vegetable protein, unsaturated fats, fiber-rich whole grains, fruits, vegetables, and legumes. They were also advised not to consume sugar-sweetened beverages, sweets, and non-nutrient-dense snack foods. Mama-DASH was designed to calorically suit the restricted weight gain recommendations (based on IOM) and to enable pregnant women to follow the nutrition guidelines specific for this population including avoidance of fish considered higher in mercury, and inclusion of calcium-rich, vitamin D-enriched dairy, or calcium-fortified nondairy products.

- The control group received the usual care that included bi-weekly newsletters and publicly available maternity website links.

In addition to the diet, all participants were encouraged to engage in at least 30 minutes of activity or walking $>10,000$ steps per day. The primary outcome of this study was GWG at 35 weeks. The study noted that pregnant women (with a prepregnancy BMI between $25-40 \mathrm{~kg} / \mathrm{m}^{2}$ ) randomized to the intervention group gained significantly less weight when compared to their counterparts in the control group. Most participants in both groups exceeded the IOM's recommended weight gain, although it was less common in the intervention group (69 percent) compared to the control group (85 percent) $(p=0.004)$. Physical activity goals were not achieved by the majority of participants. 
Summary: Of the three RCTs that assessed the relationship between Mediterranean (vs usual care), ${ }^{22}$ Mediterranean+EVOO (vs Mediterranean diet), ${ }^{23,26}$ or modified DASH (vs usual care ${ }^{25}$ diets, two trials ${ }^{22,25}$ showed that the women in the intervention group had significantly lower GWG than their counterparts in the control groups. The third trial by Assaf-Balut et al ${ }^{23,26}$ showed that women assigned to the Mediterranean Diet+EVOO tended to have lower GWG only until the end of the second trimester $(p=0.052)$. Both studies that showed an effect were conducted in either metabolically at-risk women ${ }^{22}$ or women who were overweight or obese prior to pregnancy. ${ }^{25}$ Consistent with these findings, Assaf-Balut et $\mathrm{al}^{26}$ showed that among overweight women, those in the intervention group tended to have lower GWG than those in the control group ( $p=0.076$ ) from 12 to 24 weeks; however, this difference was not observed in women who were obese prepregnancy. When considering GWG from 12 to 36-38 weeks, the total GWG was not different between the intervention and control groups. Although non-significant, there was a change in directionality for certain subgroups (i.e. normal weight, obese women) with women randomized to the intervention group gaining more weight than the control group from 12 to $36-38$ weeks. ${ }^{26}$ As part of the same trial, a sub-analysis that was restricted only to normoglycemic women showed that there was no difference in GWG from 12 to $36-38$ weeks between the intervention and control groups. ${ }^{23}$

\section{Dietary patterns assessed via reduced rank regression}

In a U.S.-based study (baseline $\mathrm{n}=764$ ), Starling et $\mathrm{al}^{11}$ arrived at DPs by "entering the residual intakes from each food group into a reduced-rank regression model." Two DPs were determined using this approach:

- Pattern 1 was characterized by a higher consumption of poultry, nuts, cheese, fruits, whole grains, added sugars, and solid fats.

- Pattern 2 was characterized by a higher consumption of eggs, starchy vegetables, solid fats, fruits, and non-whole grains and a lower consumption of dairy foods, dark-green vegetables, and whole grains.

The primary objective of this study was to assess the association between maternal DPs and newborn fat mass and adiposity, with maternal GWG as the intermediate variable. The study showed that women with greater adherence to pattern 1 (tertile 3) had significantly greater GWG ( $p$ for trend $<0.001$ ). Similarly, women with greater adherence to pattern 2 (tertile 2 and tertile 3 ) had significantly greater GWG ( $p$ for trend=0.03). However, none of the key confounders were adjusted for in the analysis.

\section{Diets based on macronutrient distributions}

As described below, one $\mathrm{RCT}^{24}$ and one prospective cohort study ${ }^{4}$ assessed the association between diets based on macronutrient distributions and GWG.

- In a U.K.-based RCT (baseline $n=183$ ), Poston et $\mathrm{al}^{24}$ randomized obese women $\left(\mathrm{BMI} \geq 30 \mathrm{~kg} / \mathrm{m}^{2}\right)$ to an intervention of dietary advice and physical activity delivered by health trainers vs. standard care. The primary outcome of the study was to assess the impact of the intervention on maternal (GDM) and neonatal (LGA) outcomes, with GWG reported as a secondary outcome. The dietary advice in the intervention group focused on increasing the consumption of foods with a low dietary glycemic index (GI), including replacing sugar-sweetened 
beverages with low $\mathrm{Gl}$ alternatives and reducing saturated fat consumption and replacing with mono- and polyunsaturated fatty acids. At the end of the intervention, there were significant differences in total fat intake between the groups, with the control group consuming an average of 35.9 percent of total energy from fat (which is outside of the AMDR), while the intervention group consumed an average of 32.5 percent of total energy from fat. The study reported that there was no significant difference in GWG between the intervention and control groups. Physical activity was a co-intervention in the study but the authors reported no difference in physical activity between the intervention and control groups.

- Tajima et $a l,{ }^{4}$ in a Japanese prospective cohort study (baseline $n=325$ ), assessed the association between tertiles of carbohydrate intake and glucose tolerance test. Based on 3-d dietary records collected from participants, the investigators assessed the average carbohydrate, protein, total fat, and saturated fatty acid intake, and calculated the percentage of total energy intake for each component. Carbohydrate intake was calculated by subtracting the percent total energy from fat and protein intake from 100 percent and participants were categorized into tertiles based on their carbohydrate intake. The study reported that women in different tertiles of carbohydrate intake also differed significantly on percent energy from total fat intake $(p<0.001)$. That is, women in the 'bottom' tertile for carbohydrate had 35.1 percent energy from total fat (which is outside of the AMDR); those in the 'middle' and 'top' tertiles for carbohydrate intake had 30.3 percent and 24.6 percent energy from total fat intake, respectively. The study also reported GWG across tertiles of carbohydrate intake (i.e., 'bottom', 'middle' and 'top') and noted that the mean weight gain per week was not significantly different across the groups. None of the key confounders were adjusted for in the analysis.

Summary: Two studies included in this body of evidence reported no significant difference in $\mathrm{GWG}^{24}$ or rate of $\mathrm{GWG}^{4}$ based on varying percentages of total energy intake from fat. Of note, neither the RCT nor the prospective cohort study manipulated percentage of energy from fat. Further, these studies were not designed to assess the relationship between macronutrient proportions and GWG. In the U.K. based trial, the control group consumed a greater percentage of total energy from fat (which was above the AMDR) when compared to the intervention group. GWG was measured as a secondary objective. While the U.K. trial did not deliberately manipulate percentage of energy from total fat, the investigators recommended replacing saturated fats with mono- and poly-unsaturated fatty acids for the intervention group, whereas routine care was recommended for the control group. The Japanese cohort study presented tertiles of carbohydrate intake and stratified GWG into categories. Women with 'bottom', 'middle' and 'top' carbohydrate intake also differed on percentage of total energy from fat. The study reported no difference in rate of GWG among these tertiles.

\section{Assessment of the evidence ${ }^{\mathrm{ix}}$}

\footnotetext{
${ }^{i x} A$ detailed description of the methodology used for grading the strength of the evidence is available on the NESR website: https://nesr.usda.gov/2020-dietary-guidelines-advisory-committee-systematic-
} 
The following conclusion statement was supported by three RCTs and 19 prospective cohort studies and was graded "limited." The individual grading elements are discussed below and summarized in Table 5 and Table 6.

"Limited evidence suggests that certain dietary patterns during pregnancy are associated with a lower risk of excessive gestational weight gain during pregnancy. These patterns are higher in vegetables, fruits, nuts, legumes, fish, and lower in added sugar, and red and processed meat"

The DPs examined were characterized by combinations of different foods and beverages. The patterns that were consistently shown to be associated with lower risk of excessive GWG commonly included the foods highlighted in the conclusion statement (i.e. higher in vegetables, fruits, nuts, legumes, fish and lower in added sugar, red and processed meat), although not all foods were part of the same pattern.

Grains and dairy products were included in a number of studies; however, the association between these foods and GWG was inconsistent. Specifically, in the 10 articles that included dairy products in a DP, some recommended consuming low-fat dairy products, while others recommended dairy consumption in moderation (without specifying percent fat), and a few other articles reverse coded it as they considered it to be detrimental. Similarly, five articles included whole/non-refined grains in a DP, of which four included it as part of a beneficial DP (meaning the DP that included whole grains was associated with a lower risk of excessive GWG). One additional article noted that avoidance of refined grains was beneficial. In three articles, it was unclear whether the participants consumed whole or refined grains and two other articles reported that participants consumed both whole and refined grains. Because of these inconsistencies, it was difficult to determine the relationship between grains or dairy and GWG.

Risk of bias was graded as limited for both RCTs and cohort studies.

- Most of the RCTs included in this body of evidence had notable flaws, as described below:

- In a RCT conducted in Spain, ${ }^{23,26}$ women randomized to both intervention and control groups received nutritional guidance to reduce the calorie content of their diet, individualized based on GWG in the first trimester BMI. In addition, women diagnosed with GDM in both the intervention and control group received treatment with insulin and/or diet. However, significantly more women in the control group were diagnosed with GDM. In the sub-analysis, ${ }^{23}$ the investigators excluded participants with gestational diabetes and assessed the effect of the diet on GWG only in normoglycemic women. Since the selection of participants into this subanalysis was based on post intervention status, this sub-analysis was treated as a "per-protocol analysis," rather than an "intention-to-treat analysis."

reviews and in Part C of the following reference: Dietary Guidelines Advisory Committee. 2020.

Scientific Report of the 2020 Dietary Guidelines Advisory Committee: Advisory Report to the Secretary of Agriculture and the Secretary of Health and Human Services. U.S. Department of Agriculture, Agricultural Research Service, Washington, DC 
- In the U.S.-based RCT, ${ }^{25}$ there were issues with adherence, which was different between the intervention and control groups.

- Outcome assessment methods were not specified in at least one RCT.

- Similar to the RCTs, the cohort studies had serious flaws in the design and conduct of the studies:

- No studies accounted for all the key confounders. Seven studies had potentially critical bias as none of the key confounders were accounted for.

- More than half of the studies had selection bias issues because selection of the participants was related to characteristics observed after the start of the exposure.

- Dietary data reflected dietary intake at a single time point during pregnancy. The exposure data were self-reported (typically collected around the end of the first trimester or later), and thus it is possible that classification of exposure status may have been impacted by the knowledge of the outcome.

- Many studies reported co-exposures which were unbalanced across treatment groups.

- Outcome data were self-reported and in a few studies reporting of outcome data may have been influenced by the knowledge of the exposure received.

- None of the studies reported having a pre-registered data analysis plan.

Consistency was graded limited for both RCTs and cohort studies.

- Among the articles that assessed DPs using an index/score method, five showed that greater adherence to a beneficial DP was associated with a lower risk of excessive GWG. 1,5,14,18,21 However, three other articles showed that greater adherence to a beneficial DP was associated with excessive GWG. 2,12,20 Seven other articles showed no association between DPs and GWG. ${ }^{6-10,13,16}$

- Four of the five cohort studies ${ }^{3,8,15,19}$ that assessed DPs using factor/cluster analysis showed an association with GWG, whereas one other study found no association between DPs and GWG. ${ }^{17}$

- One of the studies that assessed DP using reduced rank regression was associated with GWG. ${ }^{11}$

- Two RCTs showed that the intervention diet resulted in lower GWG. 22,25

- Neither of the studies that assessed the relationship between a maternal high-fat diet ( $>35$ percent total energy from fat) and GWG showed an association. ${ }^{4,24}$

Even among studies that found similar associations, the strength of the association could not be adequately assessed because of differences in study methods, including exposure and outcome assessment methodology.

The inconsistency in findings may be explained by the following: 
- Key confounders: Many of the studies that reported null findings did not adjust for any of the key confounders. This can at least partly be attributed to the fact that the studies were not designed to assess the association, but rather presented the data because they assessed GWG as a secondary outcome or a mediator in the study.

- Exposure assessment: There was heterogeneity in exposure assessment methods. Studies used different approaches to generate DPs. The foods included in each DP were also different, thus making it difficult to compare exposures across studies.

- The inconsistencies in findings between RCTs may have been due to differences in study populations. The two RCTs that found an effect only included overweight/obese women, or metabolically at-risk women. On the other hand, the RCT that showed no effect recruited healthy women, which might partly explain the inconsistency in study findings.

Directness was graded as limited for both RCTs and prospective cohort studies. Only half of the studies in this body of evidence were designed to assess the association between DPs and GWG. For the rest, GWG was a secondary measure and thus the objectives of these studies did not necessarily align with the systematic review question. Often this also meant that most or all of the key confounders were not accounted for in the analysis.

Precision was graded as limited for both RCTs and prospective cohort studies. Most of the studies, except RCTs, did not report power analyses or sample size calculations. Even among the RCTs, two trials conducted power calculations for outcomes other than GWG and thus precision with respect to the present review is difficult to evaluate. Studies included in this body of evidence generally had moderate analytic sample sizes to investigate the relationship between maternal DPs and GWG. None of the studies unduly influenced the findings of this systematic review and removing a single study from this body of evidence would not likely change the conclusions.

Generalizability was graded as limited for both RCTs and prospective cohort studies. About one-fourth of the included studies $(n=6)$, including only one RCT, were conducted in the U.S. Minority women were generally underrepresented in this body of evidence. Thirteen articles reported that their participants were predominantly White and nine others did not report race/ethnicity of the participants. With regard to SES, most women in the studies had at least some college education and participants were generally from mid-high income households. Participants were primarily adult women and none of the studies focused on adolescent mothers. Two RCTs recruited only women who were overweight/obese or had other metabolic risk factors. For the reasons stated above, it is unclear if the findings from this systematic review would be applicable to a more diverse U.S. population.

Publication bias is definitely a consideration for the systematic reviews. However, it may not be a serious concern for this body of evidence because at least a third of the studies reported non-significant findings while the others report significant findings or a mix of significant and non-significant findings. 


\section{Research recommendations}

- Include diverse populations with varying age groups and different racial/ethnic and socioeconomic backgrounds.

- Foster collaborative efforts to score dietary patterns consistently, so that they can be compared and reproduced across studies.

- Adjust for key confounding factors in observational studies, including age, race/ethnicity, SES, physical activity, anthropometry (prepregnancy BMI), smoking, history/diagnosis of gestational diabetes and gestational hypertension, and parity.

- Develop a standardized recommendation of what constitutes a 'high-fat' or 'lowcarbohydrate' dietary pattern. 


\section{Included articles}

1. Fernandez-Barres S, Vrijheid M, Manzano-Salgado CB, et al. The association of Mediterranean diet during pregnancy with longitudinal body mass index trajectories and cardiometabolic risk in early childhood. J Pediatr. 2019;206:119-127 e116. doi:10.1016/j.jpeds.2018.10.005

2. Gesteiro E, Rodriguez Bernal B, Bastida S, Sanchez-Muniz FJ. Maternal diets with low healthy eating index or Mediterranean diet adherence scores are associated with high cord-blood insulin levels and insulin resistance markers at birth. Eur J Clin Nutr. 2012;66(9):1008-1015. doi:10.1038/ejcn.2012.92

3. Okubo H, Miyake Y, Sasaki S, et al. Maternal dietary patterns in pregnancy and fetal growth in Japan: the Osaka Maternal and Child Health Study. Br J Nutr. 2012;107(10):1526-1533. doi:10.1017/S0007114511004636

4. Tajima $R$, Yachi $Y$, Tanaka $Y$, et al. Carbohydrate intake during early pregnancy is inversely associated with abnormal glucose challenge test results in Japanese pregnant women. Diabetes Metab Res Rev. 2017;33(6). doi:10.1002/dmrr.2898

5. Hillesund ER, Bere E, Haugen M, Overby NC. Development of a New Nordic Diet score and its association with gestational weight gain and fetal growth - a study performed in the Norwegian Mother and Child Cohort Study (MoBa). Public Health Nutr. 2014;17(9):1909-1918. doi:10.1017/S1368980014000421

6. Rifas-Shiman SL, Rich-Edwards JW, Kleinman KP, Oken E, Gillman MW. Dietary quality during pregnancy varies by maternal characteristics in Project Viva: a US cohort. J Am Diet Assoc. 2009;109(6):1004-1011. doi:10.1016/j.jada.2009.03.001

7. Poon AK, Yeung E, Boghossian N, Albert PS, Zhang C. Maternal dietary patterns during third trimester in association with birthweight characteristics and early infant growth. Scientifica (Cairo). 2013;2013:786409. doi:10.1155/2013/786409

8. Tielemans MJ, Erler NS, Leermakers ET, et al. A priori and a posteriori dietary patterns during pregnancy and gestational weight gain: The Generation $\mathrm{R}$ study. Nutrients. 2015;7(11):9383-9399. doi:10.3390/nu7115476

9. Sen S, Rifas-Shiman SL, Shivappa N, et al. Dietary inflammatory potential during pregnancy is associated with lower fetal growth and breastfeeding failure: results from Project Viva. J Nutr. 2016;146(4):728-736. doi:10.3945/jn.115.225581

10. Meinila J, Valkama A, Koivusalo SB, et al. Association between diet quality measured by the Healthy Food Intake Index and later risk of gestational diabetes-a secondary analysis of the RADIEL trial. Eur J Clin Nutr. 2017;71(4):555-557. doi:10.1038/ejcn.2016.275

11. Starling AP, Sauder KA, Kaar JL, Shapiro AL, Siega-Riz AM, Dabelea D. Maternal dietary patterns during pregnancy are associated with newborn body composition. J Nutr. 2017;147(7):1334-1339. doi:10.3945/jn.117.248948

12. Fulay AP, Rifas-Shiman SL, Oken E, Perng W. Associations of the dietary approaches to stop hypertension (DASH) diet with pregnancy complications in Project Viva. Eur J Clin Nutr. 2018;72(10):1385-1395. doi:10.1038/s41430-0170068-8

13. Emond JA, Karagas MR, Baker ER, Gilbert-Diamond D. Better diet quality during pregnancy is associated with a reduced likelihood of an infant born small 
for gestational age: an analysis of the prospective New Hampshire birth cohort study. J Nutr. 2018;148(1):22-30. doi:10.1093/jn/nxx005

14. Hrolfsdottir L, Halldorsson TI, Birgisdottir BE, Hreidarsdottir IT, Hardardottir H, Gunnarsdottir I. Development of a dietary screening questionnaire to predict excessive weight gain in pregnancy. Matern Child Nutr. 2019;15(1):e12639. doi:10.1111/mcn.12639

15. Wei X, He JR, Lin Y, et al. The influence of maternal dietary patterns on gestational weight gain: A large prospective cohort study in China. Nutrition. 2019;59:90-95. doi:10.1016/j.nut.2018.07.113

16. Zhu Y, Hedderson MM, Sridhar S, Xu F, Feng J, Ferrara A. Poor diet quality in pregnancy is associated with increased risk of excess fetal growth: a prospective multi-racial/ethnic cohort study. Int J Epidemiol. 2019;48(2):423432. doi:10.1093/ije/dyy285

17. Wesolowska E, Jankowska A, Trafalska E, et al. Sociodemographic, lifestyle, environmental and pregnancy-related determinants of dietary patterns during pregnancy. Int J Environ Res Public Health. 2019;16(5). doi:10.3390/ijerph16050754

18. Ancira-Moreno M, Vadillo-Ortega F, Rivera-Dommarco JA, et al. Gestational weight gain trajectories over pregnancy and their association with maternal diet quality: Results from the PRINCESA cohort. Nutrition. 2019;65:158-166. doi:10.1016/j.nut.2019.02.002

19. Maugeri A, Barchitta M, Favara G, et al. Maternal dietary patterns are associated with pre-pregnancy body mass index and gestational weight gain: results from the "Mamma \& Bambino" cohort. Nutrients. 2019;11(6). doi:10.3390/nu11061308

20. Yong HY, Mohd Shariff Z, Mohd Yusof BN, et al. Pre-Pregnancy BMI influences the association of dietary quality and gestational weight gain: The SECOST study. Int J Environ Res Public Health. 2019;16(19). doi:10.3390/ijerph16193735

21. Hillesund ER, Bere E, Sagedal LR, et al. Pre-pregnancy and early pregnancy dietary behavior in relation to maternal and newborn health in the Norwegian Fit for Delivery study - a post hoc observational analysis. Food Nutr Res. 2018;62. doi:10.29219/fnr.v62.1273

22. Al Wattar BH, Dodds J, Placzek A, et al. Mediterranean-style diet in pregnant women with metabolic risk factors (ESTEEM): A pragmatic multicentre randomised trial. PLoS Med. 2019;16(7):e1002857. doi:10.1371/journal.pmed.1002857

23. Assaf-Balut C, Garcia de la Torre N, Duran A, et al. A Mediterranean diet with an enhanced consumption of extra virgin olive oil and pistachios improves pregnancy outcomes in women without gestational diabetes mellitus: a subanalysis of the St. Carlos gestational diabetes mellitus prevention study. Ann Nutr Metab. 2019;74(1):69-79. doi:10.1159/000495793

24. Poston L, Briley AL, Barr S, et al. Developing a complex intervention for diet and activity behaviour change in obese pregnant women (the UPBEAT trial); assessment of behavioural change and process evaluation in a pilot randomised controlled trial. BMC Pregnancy Childbirth. 2013;13:148. doi:10.1186/1471-2393-13-148 
25. Van Horn L, Peaceman A, Kwasny M, et al. Dietary approaches to stop hypertension diet and activity to limit gestational weight: maternal offspring metabolics family intervention trial, a technology enhanced randomized trial. Am J Prev Med. 2018;55(5):603-614. doi:10.1016/j.amepre.2018.06.015

26. Assaf-Balut C, Garcia de la Torre N, Duran A, et al. A Mediterranean diet with additional extra virgin olive oil and pistachios reduces the incidence of gestational diabetes mellitus (GDM): A randomized controlled trial: The St. Carlos GDM prevention study. PLoS One. 2017;12(10):e0185873. doi:10.1371/journal.pone.0185873

27. Meinila J, Valkama A, Koivusalo SB, et al. Association between diet quality measured by the Healthy Food Intake Index and later risk of gestational diabetes-a secondary analysis of the RADIEL trial. Eur J Clin Nutr. 2017;71(7):913. doi:10.1038/ejcn.2017.66 
Table 3. Description of evidence on the relationship between dietary patterns during pregnancy and gestational weight gain ${ }^{x}$

Study and Participant Characteristics ${ }^{\mathrm{xi}}$

\author{
Intervention/Exposure and Outcomes
}

Results
Confounding, Study Limitations, and Summary of Findings

\section{Randomized Controlled Trials}

Index/Score

\section{Al Wattar, 201922; U.K.}

RCT, ESTEEM trial

Baseline $\mathrm{N}=1252$ Analytic $\mathrm{N}=468$

(Attrition: 63\%)

$\mathrm{n}=\sim 1190$ for all participant

characteristics

- Age: 31.1y

- Race/Ethnicity:

o White: $~ 36.0 \%$

○ Asian: $~ 43.7 \%$

o Black: $16.8 \%$

\section{Dietary Pattern(s):}

- Control: Received usual care and antenatal dietary advice as per U.K. national recommendations (Ref, $n=625$ )

- Intervention:

o High intake of nuts, EVOO, fruit, vegetables, nonrefined grains, and legumes

- Moderate to high consumption of fish

- Low to moderate intake of poultry and dairy products

- Low consumption of red meat and processed meat

\section{Significant:}

GWG (kg), $P=0.03$

- Control: $8.3 \pm 6.4$

- Intervention: $6.8 \pm 5.6$

- aOR: $-1.2,95 \% \mathrm{Cl}=(-2.2$, $0.2)$

\section{Non-significant:}

\section{Key confounders accounted for:}

Age Race/ethnicity, Prepregnancy BMI, GDM, Parity

\section{Limitations:}

- Self-reported adherence

- Dietary intake info for $\sim 40 \%$ of participants; change in diet based on intervention reported only for a sub-set of the women that were originally recruited

- Unclear how gestational weight gain was assessed and the window in which it was assessed

× ACOG: American College of Obstetricians and Gynecologists, AHEI: alternative healthy eating index, aOR: adjustd odds ration, aRR: adjusted risk ratio, BIGCS: Born in Guangzhou Cohort Study, BMI: body mass index, BW: birth weight, Cl: confidence interval, d: day, DASH: Dietary Approaches to Stop Hypertension, DHQ: diet history questionnaire, DII: Dietary Inflammation Index, DP: dietary pattern, ESTEEM: Effect of Simple, Targeted Diet in Pregnant Women With Metabolic Risk Factors on Pregnancy Outcomes, EVOO: extra virgin olive oil, FFQ: food frequency questionnaire, g: gram(s), GDM: gestational diabetes mellitus, GWG: gestational weight gain, HEl: Healthy Eating Index, HFII: Healthy Food Intake Index, hr: hour, HTN: hypertension, IFPSII: Infant Feeding Practices Study II, INMA: INfancia y Medio Ambiente, IOM: Institute of Medicine, IQR: interquartile range, kcal: kilocalories, kg: kilogram(s), MDA: Mediterranean Diet Adherence, MDQS: Maternal Diet Quality Score, Med: Mediterranean, MET: metabolic equivalent of task, mo: month, MOMFIT: Maternal Offspring Metabolics Family Intervention Trial, MoBa: Mothers and Babies cohort, MUFA: monounsaturated fatty acid, NFFD: Norwegian Fit for Delivery, NHBCS: New Hampshire Birth Cohort Study, NHS: National Health Service, NND: New Nordic Diet, NR: not reported, NS: nonsignificant, OMCHS: Osaka Maternal and Child Health Study, OMNI: optimal macronutrient intake, OR: odds ratio, PA: physical activity, PCA: principal component analysis, PCS: prospective cohort study, PE: preeclampsia, PETALS: Pregnancy Environment and Lifestyle Study, PRINCESA: Pregnancy Research on Inflammation, Nutrition and City Environments: Systematic Analyses, PREWICE: PREgnant Women of Iceland, PUFA: polyunsaturated fatty acid, Q\#: quartile, Ref: reference, RCT: randomized controlled trial, rMED: relative Mediterranean Diet, SD: standard deviation, SE: standard error, SECOST: Seremben Cohort Study, SES: socioeconomic status, SFA: saturated fatty acid, T\#: tertile, wk: week(s), y: year(s)

${ }^{x i} \pm$ indicates values of Mean \pm SD unless otherwise noted 


\section{Study and Participant}

Characteristics ${ }^{\mathrm{xi}}$

o Other: $~ 3.5 \%$

- Physical Activity ( $n=1205)$ : MET:

2,584 $\mathrm{min} / \mathrm{wk}$

- Anthropometry:

o Normal BMI: 13.9\%

○ Overweight: $16.7 \%$

o Obese: $69.4 \%$

- GDM: History: $3.2 \%$

- Gestational HTN: History of PE: $\sim 4.3 \%$

- Parity:

o Primigravida: 27.4\%

o Multigravida: 72.6\%

\section{Intervention/Exposure and Outcomes}

Results

o Avoidance of sugary drinks, fast food, and

food rich in animal fat

- Participants provided with $30 \mathrm{~g} / \mathrm{d}$ of mixed

nuts and $0.5 \mathrm{~L} / \mathrm{wk}$ of EVOO $(\mathrm{n}=627)$

at $\sim 18$ wk gestation

\section{Dietary assessment methods:}

24-hr recall used to identify changes needed in the intervention group to follow a

Mediterranean-style pattern. Validated short questionnaire (ESTEEM Q) used to assess dietary intake at 20,24, 28, 32, and 36wk
Confounding, Study Limitations, and Summary of Findings

- Metabolically at risk participants, so the findings may not be generalizable

- Weight gain was collapsed into one group and was not stratified by prepregnancy BMI

\section{Summary:}

A simple, individualized, Mediterranean-style DP supplemented with mixed nuts and EVOO moderately reduced GWG in metabolically at risk women.

\section{Outcome \& assessment methods:}

\section{GWG: Methods NR}

\section{Assaf-Balut, 2017²6; Spain}

RCT

Baseline $\mathrm{N}=1000$ Analytic $\mathrm{N}=874$ (Attrition: 13\%)

- Age: 33.0 y

- Race/Ethnicity:

- Caucasian: $68.4 \%$

- Hispanic: $28.5 \%$

o Other: $3.1 \%$

- SES:

- Education:

- Elementary: $8.8 \%$

- Secondary: $39.8 \%$

- University degree: $50.3 \%$

- Employed: $76.6 \%$

\section{Dietary Pattern(s):}

- MedDiet: Recommended diet (servings) (Ref, $n=440$ ):

$\circ \geq 2 /$ d vegetables, $\geq 3 / d$ fruit (avoiding juices), $3 /$ d skimmed dairy products, $3 / d$ wholegrain cereals, and 2-3/wk legumes - Moderate to high consumption of fish

- Low consumption of red and processed meat, avoidance of refined grains, processed baked goods, pre-sliced bread, soft drinks and fresh juices, fast foods, and precooked meals

o Recommended to walk $\geq 30 \mathrm{~min} / \mathrm{d}$ for both groups

- Advised to restrict consumption of dietary fat, including EVOO and nuts

- MedDiet + EVOO and Pistachios $(n=434)$ :

o In addition to MedDiet, recommended 40 $\mathrm{mL} / \mathrm{d}$ EVOO and 25-30g/d pistachios;

\section{Significant:}

GWG (kg) 12wk to 24-28wk gestation

- Women with prepregnancy $\mathrm{BMI}<25, \mathrm{P}=0.003$

o MedDiet $(n=330): 5.8 \pm 2.7$

$\circ$ MedDiet + EVOO and Pistachios $(n=329): 5.4 \pm$ 2.2

- **Authors write: Overall GWG was significantly lower in EVOO and

Pistachio group vs controls at 24-28 GW and at 36-38 GW $(P=0.022$ and 0.037 , respectively). At 24-28 GW, the GWG was significantly lower in all the three groups of women

\section{Key confounders accounted for:}

Age, Race/ethnicity, SES, PA, Prepregnancy BMI, Smoking, GDM, HTN, Parity

\section{Limitations:}

- The findings reported in the table are inconsistent with what is reported in the text

- After GDM diagnosis (24-28wks), women in both intervention and control groups received treatment with insulin and/or diet. Thus, women in the control group diagnosed with GDM (103/440 (23.4\%)) may have changed their diet post GDM-diagnosis. Note: there was increased olive oil intake in the control group (P-trend: 0.02).

- Women in both groups received individualized recommendations based on 


\section{Study and Participant}

Characteristics $^{\mathrm{xi}}$

- Physical Activity: Score (Walking $>5 \mathrm{~d} / \mathrm{wk}$ : Score $0: \geq 30 \mathrm{~min} / \mathrm{d},+1:>60$ $\mathrm{min} / \mathrm{d},-1:<30 \mathrm{~min} / \mathrm{d}$; Climbing stairs $>5 \mathrm{~d} /$ wk: Score 0: 4-16 floors/d, +1 : $>16$ floors/d, -1 : $<4$ floors/d): -1.4

- Anthropometry: Prepregnancy BMI: 23.1

- Smoking status: Current: $8.3 \%$

- GDM:

o GDM history: $2.8 \%$

o Family history of Type 2 DM: $25.2 \%$

- Parity:

o Primiparous: $44.3 \%$

- Second pregnancy: $32 \%$

o $>2: 23.7 \%$

\section{Intervention/Exposure and Outcomes}

study provided 1L/wk EVOO and 150g/wk roasted pistachios throughout the pregnancy.

at $12-14 \mathrm{wks}$

\section{Dietary assessment methods:}

Participants randomized to either MedDiet or MedDiet + EVOO and Pistachios at 8-12wk gestation

\section{Outcome \& assessment methods:}

Pregestational body weight was self-reported and registered at 12-14 wks (Visit 1). Weight measured at each visit $(1,2$ and 3$)$. GWG evaluated at 24-28 and 36-38wk gestation (in relation to weight at Visit 1).

\section{Results}

stratified by BMI (<25, 2529.9 and $\geq 30$ ).

\section{Non-significant:}

GWG (kg) 12wk to 24-28wk gestation

- Total Sample, $P=0.052$

o MedDiet: $5.6 \pm 2.8$

0 MedDiet + EVOO and

Pistachios: $5.2 \pm 2.5$

- Women with prepregnancy BMI 25-29.9, $P=0.076$

- MedDiet $(n=88): 5.1 \pm 3.0$

$\circ$ MedDiet + EVOO and Pistachios ( $n=85): 4.7 \pm$ 3.4

- Women with prepregnancy $\mathrm{BMI} \geq 30, \mathrm{P}=\mathrm{NS}$

GWG (kg) 12wk to 36-38wk gestation

- Total Sample, P=NS

- Women with prepregnancy $\mathrm{BMI}<25, \mathrm{P}=0.096$

- MedDiet $(n=300): 9.9 \pm 3.9$

$\circ$ MedDiet + EVOO and Pistachios $(n=329): 10.6 \pm$ 4.0

- Women with prepregnancy BMI 25-29.9, $P=0.066$

o MedDiet $(n=88): 8.8 \pm 4.5$

MedDiet + EVOO and

Pistachios $(n=85): 8.3 \pm 6.5$

\section{Confounding, Study Limitations, and}

Summary of Findings

their first trimester BMI to reduce calorie content, when GWG goal is exceeded.

\section{Summary:}

Consuming a MedDiet with EVOO and Pistachios vs MedDiet alone resulted in less GWG at 24-28wk, particularly among women with prepregnancy $\mathrm{BMI}<25$. There were no statistically signficant differences in GWG between groups at 36-38wk. 
Study and Participant

Characteristics $^{\mathrm{xi}}$

Intervention/Exposure and Outcomes

Results

Confounding, Study Limitations, and

Summary of Findings

Women with prepregnancy

$\mathrm{BMI} \geq 30, \mathrm{P}=\mathrm{NS}$

\section{Assaf-Balut, 2019²3; Spain}

RCT

Baseline N=1000 Analytic N=697

- Age: $~ 33.0 y$

- Race/Ethnicity:

- Caucasian: $67.5 \%$

o Hispanic: $29.7 \%$

○ Other: $2.8 \%$

- SES:

- Education:

- University degree: $50.8 \%$

- Employed: $77.3 \%$

- Physical Activity Score $>0$ (Walking $>5 \mathrm{~d} / \mathrm{wk}$ : Score 0: $\geq 30 \mathrm{~min} / \mathrm{d},+1$ : >60 $\mathrm{min} / \mathrm{d},-1$ : $<30 \mathrm{~min} / \mathrm{d}$; Climbing stairs $>5 \mathrm{~d} /$ wk: Score 0: 4-16 floors/d, +1 : $>16$ floors/d, -1 : $<4$ floors/d): $11.6 \%$

- Anthropometry: Prepregnancy BMI: 22.6

- Smoking status: Current: $7.9 \%$

- GDM:

○ GDM history: $2.2 \%$

o Family history of Type 2 DM: $4.2 \%$ - $0 \%$ GDM

- Parity:

o Primiparous: $43.2 \%$

○ Second pregnancy: $32.3 \%$

o $>2: 24.5 \%$

\section{Dietary Pattern(s):}

- MedDiet: Recommended diet (servings) (Ref, $n=337$ ):

$\circ \geq 2 / d$ vegetables, $\geq 3 / d$ fruit (avoiding juices), $3 /$ d skimmed dairy products, $3 / d$ wholegrain cereals, and 2-3/wk legumes

- Moderate to high consumption of fish

- Low consumption of red and processed meat, avoidance of refined grains, processed baked goods, pre-sliced bread, soft drinks and fresh juices, fast foods, and precooked meals

- Recommended to walk $\geq 30 \mathrm{~min} / \mathrm{d}$ for both groups

- Advised to restrict consumption of dietary fat, including EVOO and nuts

- MedDiet + EVOO and Pistachios ( $n=360$ ): o In addition to MedDiet, recommended 40 $\mathrm{mL} / \mathrm{d}$ EVOO and 25-30g/d pistachios; study provided $1 \mathrm{~L} /$ wk EVOO and $150 \mathrm{~g} / \mathrm{wk}$ roasted pistachios throughout the pregnancy.

at $12-14 \mathrm{wks}$

\section{Dietary assessment methods:}

Participants randomized to either MedDiet or MedDiet + EVOO and Pistachios at 8-12wk gestation

\section{Outcome \& assessment methods:}

Prepregnancy weight self-reported at baseline (12-14wk gestation). Weight

\section{Significant:}

\section{Non-significant:}

GWG (kg) 12wk to 36-38wk gestation, $\mathrm{P}=\mathrm{NS}$

- MedDiet $(n=337): 10.29 \pm$ 3.97

- MedDiet + EVOO and Pistachios $(n=360): 10.68 \pm$ 4.63

GWG adequacy, $\mathrm{P}=\mathrm{NS}$

- Excessive

o MedDiet $(n=337): 26.7 \%$

$\circ$ MedDiet + EVOO and

Pistachios ( $n=360): 27.5 \%$

- Adequate

○ MedDiet ( $\mathrm{n}=337$ ): $70.9 \%$

$\circ$ MedDiet + EVOO and

Pistachios ( $n=360): 69.2 \%$

- Inadequate

o MedDiet $(n=337): 2.4 \%$

$\circ$ MedDiet + EVOO and

Pistachios $(n=360): 3.3 \%$

\section{Key confounders accounted for:}

Age, Race/ethnicity, SES, PA, Prepregnancy BMI, Smoking, GDM, Parity

\section{Limitations:}

- Women in both groups received individualized recommendations based on their first trimester BMI to reduce calorie content, when GWG goal is exceeded.

- Risk of selective reporting

\section{Summary:}

Consuming a MedDiet with EVOO and Pistachios vs MedDiet alone resulted in no difference in GWG or GWG adequacy, particularly among normoglycemic women. 
last weight recorded before delivery). GWG

reported at 36-38wk in relation to baseline.

Adequate GWG defined according to

prepregnancy BMl: $<25: 9 \mathrm{~kg}, 25-29: 9.6 \mathrm{~kg}$,

$30-34.9: 3 \mathrm{~kg}$, and $\geq 35: 0 \mathrm{~kg}$. Weight gain 3

$\mathrm{kg}$ below designated target categorized as

insufficient. Weight gain $3 \mathrm{~kg}$ above

designated target categorized as excessive.

\section{Van Horn, 2018 25 ; U.S. RCT, MOMFIT}

Baseline $\mathrm{N}=281$ Analytic $\mathrm{N}=280$

(Attrition: 0\%)

- Age: 33.6y

- Race/Ethnicity:

o Hispanic: 21.4\%

o White: $63.3 \%$

- Black/African American: 19.2\%

o Other: 17.4\%

- SES:

- Education:

- sHigh school diploma/GED: $4.6 \%$

- 1-3 years' college: $\sim 15.7 \%$

- College degree: $~ 39.1 \%$

- Postgraduate work: $~ 40.2 \%$

- Marital status:

- Married: 83.3\%

- Living with significant other: 9.6\%

- Not married: $~ 7.1 \%$

- Total family income:

- $<\$ 20,000: \sim 4.6 \%$

- $\geq \$ 200,000: \sim 17.4 \%$

- Physical Activity (Median time/wk):

\section{Dietary Pattern(s):}

- Control: Received usual-care, including biweekly newsletters and publicly available maternity website links via email (Ref, $\mathrm{n}=140$ )

- Intervention: Mama-DASH diet

o Encouraged low-fat dairy products, fish, skinless poultry, lean meat and vegetable protein, unsaturated fats, fiber-rich whole grains, fruits, vegetables, and legumes

- Discouraged sugar-sweetened beverages, sweets, and non-nutrient-dense snack foods

- Calorically suited to restricted GWG recommendations and followed nutrition guidelines for pregnant women, including avoidance of fish considered higher in mercury, inclusion of calcium-rich, vitamin D-enriched dairy, or calcium-fortified nondairy products

- Received 3 individual and 6 group diet and physical activity counseling sessions by phone and webinar $(n=140)$

at $15 \mathrm{wk}$ gestation

Dietary assessment methods:

\section{Significant:}

GWG (kg), $P=0.02$

- Control: $12 \pm 6$

- Intervention: $10 \pm 6$

Exceeded GWG guidelines, $\mathrm{P}=0.004$

- Control: $84.4 \%$

- Intervention: $68.6 \%$

GWG (kg), P=0.01

- Prepregnancy BMI >30

o Control: $26 \pm 14$

o Intervention: $20 \pm 12$

Exceeded GWG guidelines, $\mathrm{P}=0.001$

- Prepregnancy BMI >30

- Control: 83\%

o Intervention: $60 \%$

\section{Key confounders accounted for:}

Age, Race/ethnicity, Prepregnancy BM

\section{Limitations:}

- Self-reported diet and exercise

- Lower adherence to intervention than expected

- Analyses differ between protocol and publication

\section{Summary:}

The MOMFIT intervention, including adherence to the Mama-DASH DP, significantly reduced GWG in obese women.

\section{Non-significant:}




\section{Study and Participant}

Characteristics ${ }^{\mathrm{xi}}$

o Moderate activities (3-6 METs):

$$
\sim 4.5 \mathrm{hr}
$$

$\circ$ Vigorous activities (7-12 METs): $\sim 0.55 \mathrm{hr}$

- Anthropometry:

o Prepregnancy BMI: 31

o Prepregnancy obesity: $54.8 \%$

- Smoking status: $0 \%$

- Parity: Nulliparous: $47.0 \%$

- Sleep duration $>7$ hrs: $~ 50.5 \%$

\section{Intervention/Exposure and Outcomes}

Two self-reported 24-hour recalls collected prior to randomization and at $35 \mathrm{wks}$ using the ASA24 system. Nutrient and food group intakes averaged at each time point with and without vitamin/mineral supplements, and then further averaged for group means by randomization status. Adherence to DASH diet scored 0-9 and 8-40 using different methodologies. Diet quality also assessed using the $\mathrm{HEI}-2010$, with scores ranging from 0-100.

All participants received the 2008 Physical Activity Guidelines for Americans and recommendations from the ACOG.

\section{Outcome \& assessment methods:}

GWG calculated as difference between selfreported prepregnancy weight and weight measured by blinded, trained staff at 35wk (weight closest to this time extracted from clinic charts or taken from previous study visits if no 35wk measure available). GWG adequcy determined by IOM 2009 recommendations.
Confounding, Study Limitations, and Summary of Findings
GWG (kg), stratified by

prepregnancy BMI, Median (IQR), $\mathrm{P}=0.49$

- Prepregnancy BMI >25-30,

$$
\mathrm{P}=\mathrm{NS}
$$

Exceeded GWG guidelines, Median (IQR), $\mathrm{P}=0.33$

Prepregnancy BMI

Overweight, $\mathrm{P}=\mathrm{NS}$

\section{Prospective Cohort Studies}

\section{Factor Analysis and PCA}

\section{Maugeri, 2019'19; Italy}

\section{PCS, Mamma \& Bambino}

Baseline $\mathrm{N}=232$ Analytic $\mathrm{N}=232$ (Attrition: 0\%)

\section{Dietary Pattern(s)}

- Prudent dietary pattern characterized by a high intake of boiled potatoes, cooked vegetables, legumes, pizza and soup

- Western dietary pattern characterized by high intake of red meat, fries, dipping sauces, salty snacks and alcoholic drinks.

\section{Significant:}

GWG $(\mathrm{kg})$, All women:

- Western Diet

oT1: Ref

o Trend T1 to T3: $\beta=1.217$,

$\mathrm{SE}=0.487, \mathrm{P}=0.013$

\section{Key confounders accounted for:}

Age, SES, Smoking, GDM, HTN, Parity

\section{Limitations:}

- Accounted for total energy intake 


\section{Study and Participant}

Characteristics $^{\mathrm{xi}}$

- Median Age: 37.3y

- Race/Ethnicity: NR

- SES:

○ $\leq 8 \mathrm{y}$ of school: $\sim 19.4 \%$

○ Working: 58.3\%

- Anthropometry: Prepregnancy BMI: $\sim 22.6$

- Smoking status: $18.1 \%$

- GDM: 0\%

- Gestational HTN: 0\%

\section{Intervention/Exposure and Outcomes}

at $\sim 16$ wk gestation

\section{Dietary assessment methods:}

Dietary assessment performed at $\sim 16 \mathrm{wk}$ gestation using a validated 95 -item semiquantitative FFQ, which referred to the previous month. Items from the FFQ were classified into 39 predefined food groups using PCA

\section{Outcome \& assessment methods:}

GWG $(\mathrm{kg})$ calculated by subtracting selfreported prepregnancy weight from weight measured at delivery

\section{Confounding, Study Limitations, and} Summary of Findings

GWG (kg), Prepregnancy BMI Underweight

- Prudent Diet

○ Trend T1 to T3: $\beta=4.127$, $\mathrm{SE}=1.722, \mathrm{P}=0.048$

GWG (kg), Prepregnancy BMI Overweight

- Prudent Diet

$\circ$ T1: Ref

$\circ \mathrm{T} 2: \beta=-7.975, \mathrm{SE}=2.672$, $P=0.010$

$\circ \mathrm{T} 3: \beta=-9.736, \mathrm{SE}=4.302$, $\mathrm{P}=0.037$

$\circ$ Trend T1 to T3: $\beta=-4.209$, $S E=1.635, P=0.016$

GWG (kg), Prepregnancy BMI Obese

- Prudent Diet

o Trend T1 to T3: $\beta=-7.356$ $\mathrm{SE}=2.304, \mathrm{P}=0.031$

- Western Diet

$\circ$ T1: Ref

○ T3: $\beta=13.701$, SE $=0.887$, $\mathrm{P}=0.041$

- Trend T1 to T3: $\beta=7.363$, $\mathrm{SE}=1.808, \mathrm{P}=0.005$

\section{Non-significant:}

GWG (kg), All women, $\mathrm{P}=0.830$

- Prudent Diet, $\mathrm{P}=\mathrm{NS}$
- Could not investigate the effect of DP on GWG trajectories

- Limited sample size in underweight and obese groups

- PCA-derived dietary patterns only explained $15.55 \%$ of total variance among food groups

- Selection into the analysis was related to exposure and outcome and not adjusted for in the analysis; Start of follow up and exposure did not coincide and a potentially important amount of follow-up time is missing from analyses

- Dietary data reflected first trimester intake, when mothers may have been prone to morning sickness

- Outcome measure was subjective and assessed by participants

- No pre-registered data analysis plan

\section{Summary:}

Adherence to a Western DP is positively associated with GWG, especially among obese women. Adherence to a prudent DP is positively associated with GWG among underweight women and negatively associated with GWG among overweight and obese women. 


\section{Study and Participant}

Characteristics $^{\mathrm{xi}}$
Confounding, Study Limitations, and

Summary of Findings

- Western Diet, Median

(IQR), $\mathrm{P}=0.056$

OT1: 11.5 (7.2)

○ T2: $13.0(7.0)$

○ T3: $13.0(9.0)$

GWG Adequacy

- Prudent Diet, $P=0.823$

- No difference in the prevalence of inadequate, adequate, and excessive GWG among tertiles of adherence

- Western Diet, $P=0.162$

o No difference in the

prevalence of inadequate, adequate, and excessive

GWG among tertiles of adherence

GWG $(\mathrm{kg})$ among all women, and those with Prepregnancy underweight or normal weight:

- Prudent Diet

No association with T2 or T3 (vs T1) adherence and GWG, and no significant trend.

- Western Diet

- No association with T2 or T3 (vs T1) adherence and GWG, and no significant trend. 
GWG (kg), Prepregnancy BMI

Overweight

- Western Diet

- No association with T2 or

T3 (vs T1) adherence and

GWG, and no significant

trend.

GWG (kg), Prepregnancy BMI

Obese

- Prudent Diet

oT1: Ref

$\circ \mathrm{T} 2$ vs $\mathrm{T} 1, \mathrm{P}=\mathrm{NS}$

$\circ$ T3: $\beta=-10.730, S E=4.156$,

$P=0.061$

- Western Diet

oT1: Ref

$\circ \mathrm{T} 2$ vs $\mathrm{T} 1, \mathrm{P}=\mathrm{NS}$

\section{Okubo, 2012³; Japan \\ PCS, OMCHS}

Baseline $\mathrm{N}=803$ Analytic $\mathrm{N}=803$

(Attrition: 0\%)

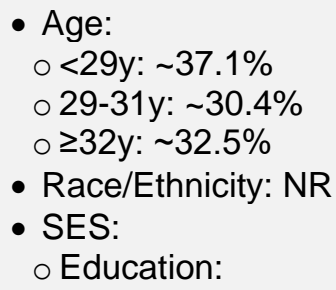

- Race/Ethnicity: NR

- SES:

o Education:

\section{Dietary Pattern(s):}

- Meat and eggs pattern: high intake of beef and pork, processed meat, chicken, eggs, butter and dairy products $(n=326)$

-Wheat products pattern: high intake of bread, confectioneries, fruit and vegetable juice, and soft drinks $(n=303)$

- Rice, fish, and vegetables pattern: high intake of rice, potatoes, nuts, pulses, fruits, green and yellow vegetables, white vegetables, mushrooms, seaweeds, Japanese and Chinese tea, fish, shellfish, sea products, miso soup and saltcontaining seasoning $(n=174)$

\section{Significant:}

GWG $(\mathrm{kg})$, Mean $(95 \% \mathrm{Cl})$, $\mathrm{P}=0.01$

- Meat and eggs: 10.0 (9.7, 10.3)

- Wheat products: $10.2(9.8$, 10.6)

- Rice, fish and vegetables: $9.3(8.8,9.7)$

$\circ$ Meat and eggs vs. wheat products: NS

$\circ$ Meat and eggs vs. rice, fish and veg.: NS

\section{Key confounders accounted for:}

None

\section{Limitations:}

- Questionnaire not validated in pregnant women and may not reflect total food and nutrient intake

- Self-administered questionnaire used to assess potential confounders were developed for this study not validated

- Self-reported outcome data collected 2-9 mo postpartum

- Supplement intake: Sig higher in rice, fish and vegetables ( $29 \%$ vs. $\sim 19 \%$ in other groups) 


\begin{tabular}{|c|c|c|c|}
\hline $\begin{array}{l}\text { Study and Participant } \\
\text { Characteristics }^{\mathrm{xi}}\end{array}$ & Intervention/Exposure and Outcomes & Results & $\begin{array}{l}\text { Confounding, Study Limitations, and } \\
\text { Summary of Findings }\end{array}$ \\
\hline 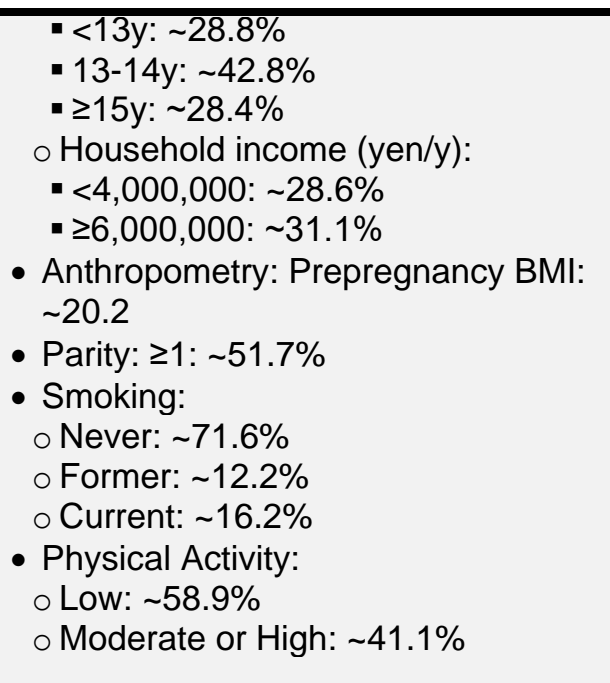 & $\begin{array}{l}\text { at } 18.0 w k \text {, Range: } 5 \text { to 39wk gestation } \\
\text { Dietary assessment methods: } \\
\text { Self-administered, comprehensive, } 150 \text {-item } \\
\text { diet history questionnaire (DHQ), validated in } \\
\text { Japanese men and women (not specifically } \\
\text { pregnant women) (see Sasaki, } 2000 \text { and } \\
\text { Sasaki, 1998) } \\
\text { Patterns identified by K-means cluster } \\
\text { analysis } \\
\text { Outcome \& assessment methods: } \\
\text { Maternal weight gain was obtained using a } \\
\text { survey collected 2-9 mo postpartum }\end{array}$ & $\begin{array}{l}\text { Wheat vs. rice, fish and } \\
\text { veg: } P<0.05\end{array}$ & $\begin{array}{l}\text { - Follow-up time varied (potentially widely) } \\
\text { across participants } \\
\text { - Important co-exposures imbalanced across } \\
\text { groups and could have likely to impacted the } \\
\text { outcome } \\
\text { - Missingness by exposure NR } \\
\text { - No pre-registered data analysis plan } \\
\text { Summary: } \\
\text { Adherence to a wheat products dietary patterns } \\
\text { was associated with significantly greater weight } \\
\text { gain when compared to rice, fish, and } \\
\text { vegetables DPs }\end{array}$ \\
\hline Tielemans, $2015^{8}$; Netherlands & Dietary Pattern(s): & Significant: & Key confounders accounted for: \\
\hline $\begin{array}{l}\text { Baseline } N=3374 \text { Analytic } N=2748 \\
\text { (Attrition: } 33 \%) \\
\text { Women excluded due to missing diet } \\
\text { data }(n=538) \text {, weight data }(n=5) \text {, } \\
\text { multiple pregnancy }(n=53) \text {, induced } \\
\text { abortion }(n=8) \text {, intrauterine fetal death } \\
\text { ( } n=16) \text {, loss-to-follow up }(n=3) \text {, } \\
\text { prepregnancy underweight }(n=100) \text {, } \\
\text { leaving } 3374 \text { women for the current } \\
\text { analysis. }\end{array}$ & $\begin{array}{l}\text { - Vegetable, Oil and Fish Pattern: higher } \\
\text { intake of vegetables, high fat dairy } \\
\text { products, cereals (both low and high fiber), } \\
\text { fish and shellfish, eggs and egg products, } \\
\text { vegetable oils, coffee and tea, alcoholic } \\
\text { beverages and legumes } \\
\text { - Nuts, High-Fiber Cereals and Soy Pattern: } \\
\text { higher intake of potatoes and other tubers, } \\
\text { fruits, high and low fat dairy products, high } \\
\text { fiber cereals, meat and meat products, fish } \\
\text { and shellfish, coffee and tea, sugar- } \\
\text { containing beverages, light soft drinks, nuts, } \\
\text { seeds and olives, and soy products } \\
\text { Margarine, Sugar and Snacks Pattern: } \\
\text { higher intake of potatoes and other tubers, } \\
\text { high fat dairy products, low and high fiber } \\
\text { cereals, meat and meat products, }\end{array}$ & $\begin{array}{l}\text { Weekly GWG adequacy } \\
\text { - Inadequate }(\mathrm{n}=437, \text { Ref: } \\
\text { Adequate, } \mathrm{n}=753) \\
\text { O Margarine, Sugar, and } \\
\text { Snacks Pattern } \mathrm{Q} 4 \text { vs } \mathrm{Q} 1 \text { : } \\
\text { OR=1.49, 95\% } \mathrm{Cl}=(1.05 \text {, } \\
2.11) \\
\text { - Excessive }(\mathrm{n}=1555 \text {, Ref: } \\
\text { Adequate, } \mathrm{n}=753) \\
\text { Margarine, Sugar, and } \\
\text { Snacks Pattern } \mathrm{Q} 4 \text { vs } \mathrm{Q} 1 \text { : } \\
\text { OR=1.32, 95\% } \mathrm{Cl}=(1.01 \text {, } \\
1.720\end{array}$ & $\begin{array}{l}\text { Age, Race/ethnicity, SES, Prepregnancy BMI, } \\
\text { Smoking, Parity } \\
\text { Limitations: } \\
\text { - Accounted for total energy intake } \\
\text { - Start of follow up and start of exposure did not } \\
\text { coincide } \\
\text { - Self-reported diet, prepregnancy weight, and } \\
\text { maximum pregnancy weight } \\
\text { - No pre-registered data analysis plan } \\
\text { Summary: } \\
\text { None of the a posteriori-derived DPs were } \\
\text { associated with GWG. }\end{array}$ \\
\hline
\end{tabular}




\section{Study and Participant}

Characteristics $^{\mathrm{xi}}$

- Race/Ethnicity: $100 \%$ of Dutch ancestry

- SES:

o Education:

- Low and midlow: 18.2\%

- Midhigh: 51.5\%

- High: $30.4 \%$

o Household income <2200 Euro/mo: 28.3\%

- Anthropometry: Prepregnancy BMI: $\sim 24.7$

- Smoking status:

o Never in pregnancy: $74.4 \%$

o Until pregnancy known: $8.3 \%$

o During pregnancy: $\sim 17.4 \%$

- Parity:

o 0: $59.8 \%$

$\circ \geq 1: \sim 40.2 \%$

- Alcohol:

o Never in pregnancy: $36.6 \%$

o Until pregnancy known: 16.5\%

o During pregnancy: $46.9 \%$

\section{Intervention/Exposure and Outcomes}

margarine and butter, sugar and

confectionary and cakes, snacks, sugar-

containing beverages, condiments and

sauces, nuts, seeds and olives

at $\sim 13.4$ wk gestation

Higher adherence to each pattern indicates a diet characterized by higher intake of those food groups.

\section{Dietary assessment methods:}

Dietary intake assessed at $\sim 13.4$ wk gestation using a validated, 293-item FFQ that covered dietary intake over the previous 3 months. A posteriori-derived dietary patterns identified using PCA with Varimax rotation. Adherence scores for each participant and each pattern calculated by individual sum of the intake of the 23 food groups, weighted with their factor loadings and standardizing those weighted sums to have mean zero and standard deviation one.

\section{Outcome \& assessment methods:}

Self-reported prepregnancy weight collected at enrollment. Staff measured weight at 3 study visits: 12.9wk (first visit), 20.4wk (second visit), and $30.2 \mathrm{wk}$ (third visit). At 6wk postpartum, women self-reported maximum weight during pregnancy.

GWG in different phases of pregnancy was calculated mid-pregnancy GWG (weight at the second visit minus weight the first visit, divided by follow-up duration (g/week), $n=$ 2748), late-pregnancy GWG (weight at the third visit minus weight at the second visit,
Results

Confounding, Study Limitations, and

Summary of Findings
Mid-Pregnancy Weight Gain

(g/wk)

- Prepregnancy Normal

Weight $(\mathrm{n}=2079)$

- Vegetable, Oil, and Fish Pattern, $P=N S$ per SD increase in DP score

- Nuts, High-Fiber Cereals and Soy Pattern, $\mathrm{P}=\mathrm{NS}$

- Margarine, Sugar and Snacks Pattern, $\mathrm{P}=\mathrm{NS}$

- Prepregnancy Overweight Women $(n=669)$

- Vegetable, Oil, and Fish Pattern, $\mathrm{P}=\mathrm{NS}$ per SD increase in DP score

- Nuts, High-Fiber Cereals and Soy Pattern, $\mathrm{P}=\mathrm{NS}$

- Margarine, Sugar and Snacks Pattern, $\mathrm{P}=\mathrm{NS}$

Late-Pregnancy Weight Gain (g/wk)

- Prepregnancy Normal Weight Women $(n=2384)$

- Vegetable, Oil, and Fish Pattern, $P=N S$ per SD increase in DP score

- Nuts, High-Fiber Cereals and Soy, $\mathrm{P}=\mathrm{NS}$

- Margarine, Sugar and Snacks Pattern, $\mathrm{P}=\mathrm{NS}$

- Prepregnancy Overweight Women $(n=774)$ 


\section{Study and Participant}

Characteristics $^{\mathrm{xi}}$

\section{Intervention/Exposure and Outcomes \\ Results}

divided by follow-up duration ( $\mathrm{g} /$ week), $\mathrm{n}=$ 3158), and GWG until early-third trimester (weight at the third visit minus prepregnancy weight, divided by follow up duration (g/week), $\mathrm{n}=2815$ ).

Women's total GWG (maximum pregnancy weight minus prepregnancy weight, $n=1917$ ) and GWG rate between visits 1 and 3 was used to classify their GWG into inadequate, adequate, or excessive GWG according to IOM (2009) recommendations. $\circ$ Vegetable, Oil, and Fish Pattern, $\mathrm{P}=\mathrm{NS}$ per SD increase in DP score

- Nuts, High-Fiber Cereals and Soy, $\mathrm{P}=\mathrm{NS}$

- Margarine, Sugar and Snacks Pattern, $\mathrm{P}=\mathrm{NS}$

GWG adequacy:

- Inadequate GWG ( $n=459$, Ref: Adequate, $\mathrm{n}=632$ )

- Vegetable, Oil and Fish Pattern, $\mathrm{P}=\mathrm{NS}$ per SD increase in DP score - Nuts, High-Fiber Cereals and Soy Pattern, $\mathrm{P}=\mathrm{NS}$ - Margarine, Sugar and Snacks Pattern, $\mathrm{P}=\mathrm{NS}$

- Excessive GWG ( $\mathrm{n}=826$, Ref: Adequate, $n=632$ ) - Vegetable, Oil and Fish Pattern, $P=N S$ per SD increase in DP score

○ Nuts, High-Fiber Cereals and Soy Pattern, $\mathrm{P}=\mathrm{NS}$

- Margarine, Sugar and Snacks Pattern, $P=N S$

Weekly GWG adequacy

- Inadequate $(n=437$, Ref: Adequate, $n=753$ )

- Vegetable, Oil, and Fish Pattern, $P=N S$ per SD increase in DP score
Confounding, Study Limitations, and Summary of Findings 


\section{Study and Participant}

Characteristics $^{\mathrm{xi}}$
Intervention/Exposure and Outcomes

Results

o Nuts, High-Fiber Cereals

and Soy Pattern, $\mathrm{P}=\mathrm{NS}$

- Margarine, Sugar, and

Snacks Pattern, $\mathrm{P}=\mathrm{NS}$

- Excessive ( $n=1555$, Ref:

Adequate. $\mathrm{N}=753$ )

- Vegetable, Oil, and Fish

Pattern, $P=N S$ per SD

increase in DP score

- Nuts, High-Fiber Cereals

and Soy Pattern, $\mathrm{P}=\mathrm{NS}$

Margarine, Sugar, and

Snacks Pattern, $\mathrm{P}=\mathrm{NS}$

Tielemans, 2015 continued

\section{Significant:}

Sensitivity Analysis (excludes

women with GDM or

hypertensive disorders of pregnancy)

GWG until early-third

trimester ( $\mathrm{g} / \mathrm{wk}$ )

- Prepregnancy Normal

Weight Women $(n=1937)$

- Vegetable, Oil and Fish

Pattern, $P<0.01$ per SD

increase in DP score

- Q1: Ref

- Q2: $\beta=20,95 \%$ Cl: (3,

36), $P<0.05$

- Q3: $\beta=7,95 \% \mathrm{Cl}:(-10$

- Q4: $\beta=27,95 \% \mathrm{Cl}:(11$,

44), $P<0.05$
Confounding, Study Limitations, and

Summary of Findings

\section{Non-significant:}

\section{Key confounders accounted for:}

Age, Race/ethnicity, SES, Prepregnancy BMI, Smoking, GDM, HTN, Parity

\section{Summary:}

Among women with normal prepregnancy weight, higher adherence to the Vegetable, Oi and Fish Pattern was associated with greater GWG. 


\section{Study and Participant}

Characteristics $^{\mathbf{x}}$
Results

Sensitivity Analysis (excludes

women with GDM or

hypertensive disorders of

pregnancy)

GWG until early-third

trimester $(\mathrm{g} / \mathrm{wk})$

- Prepregnancy Norma

Weight Women $(n=1937)$

- Nuts, High-Fiber Cereals

and Soy, $\mathrm{P}=\mathrm{NS}$ per SD

increase in DP score

- Margarine, Sugar, and

Snacks, $\mathrm{P}=\mathrm{NS}$

- Prepregnancy Overweight Women $(n=532)$

- Vegetable, Oil and Fish

Pattern, $\mathrm{P}=\mathrm{NS}$ per SD

increase in DP score

- Nuts, High-Fiber Cereals

and Soy Pattern, $\mathrm{P}=\mathrm{NS}$

- Margarine, Sugar and

Snacks Pattern, $\mathrm{P}=\mathrm{NS}$
Confounding, Study Limitations, and

Summary of Findings

\section{Wei, 201915; China} PCS, BIGCS

Baseline $\mathrm{N}=5,733$ Analytic $\mathrm{N}=5,733$

- Age: 29.1y

- Race/Ethnicity: NR

- SES:

o Educational level:

\section{Dietary Pattern(s)}

Six dietary patterns were generated according to the food groups predominant in each cluster.

- Cereals $(n=872)$

- Vegetables $(n=1147)$

- Meats $(n=927)$

- Fruits $(n=640)$

- Fish, beans, nuts, and yogurt ( $n=1130)$

- Milk and milk powder $(n=817)$

\section{Significant:}

GWG (kg), $P=0.007$

- Cereals: Ref

- Fruits: $\beta=0.592,95 \% \mathrm{Cl}$ : $(0.166,1.018)$

GWG rate $(\mathrm{kg} / \mathrm{wk}), \mathrm{P}=0.007$

- Cereals: Ref

\section{Key confounders accounted for:}

Age, SES, Prepregnancy BMI, Parity

Limitations:

- Food quantity and portion size not documented in the FFQ

- Adjusted for post-exposure variables

- Selection into analysis related to outcome 


\section{Study and Participant}

Characteristics $^{\mathrm{x}}$

- High school or below: 8.0\%

- Vocational/technical college: $23.6 \%$

- Undergraduate: $55.5 \%$

- Postgraduate: $12.9 \%$

o Income (Yuan/mo; $n=5605)$ :

- $\leq 1500: 9.3 \%$

- $\geq 9001: 16.9 \%$

- Anthropometry: Prepregnancy BMI:

$20.4 \pm 2.6$

- Smoker: $29.7 \%$

- Parity: Primiparous: $88.2 \%$

\section{Intervention/Exposure and Outcomes}

at 24-27wk gestation

\section{Dietary assessment methods:}

A validated, 64-item FFQ used to assess diet Individual food items were combined into 30 groups by similar nutrient profile or culinary use. Contribution (\%) of every food group was calculated for each participant. Cluster analysis with $\mathrm{K}$-means method was conducted to generate six dietary patterns based on foods highly consumed and distribution of foods within clusters.

\section{Outcome \& assessment methods:}

Prepregnancy weight self-reported, while weight during pregnancy extracted from medical records. Total GWG $(\mathrm{kg})$ calculated as difference in weight between prepregnancy and delivery. Total GWG rate ( $\mathrm{kg} / \mathrm{wk}$ ) calculated by dividing total GWG by total gestational wks. Second trimester GWG rate $(\mathrm{kg} / \mathrm{wk})$ calculated as difference in weight before $28 \mathrm{wk}$ and weight after $13 \mathrm{wk}$, divided by wks between the two measures. Third trimester GWG rate $(\mathrm{kg} / \mathrm{wk})$ calculated as difference between pre-delivery weight and weight at 28wk, divided by wks between the two measures.

\section{Results}

- Fruits: $\beta=0.015,95 \% \mathrm{Cl}$ :

$(0.004,0.026)$

Second Trimester GWG rate (kg/wk), $\mathrm{P}<0.05$

- Cereals: Ref, $n=821$

- Fish, beans, nuts, and yogurt: $\beta=0.024,95 \% \mathrm{Cl}$ : (0.001, 0.048), $\mathrm{n}=658$

\section{GWG adequacy}

- Inadequate GWG

o Cereals: Ref

- Fish, beans, nuts, and yogurt: $\mathrm{OR}=0.797,95 \% \mathrm{Cl}$ $(0.638,0.997)$

- Excessive GWG

o Cereals: Ref

o Fruits: OR=1.393, 95\% Cl: $(1.101,1.763)$

\section{Non-significant:}

GWG (kg), P=NS

- Cereals: Ref

- Vegetables

- Meats

- Fish, beans, nuts, and yogurt: $\beta=0.341,95 \% \mathrm{Cl}$ : ($0.017,0.699), P=0.062$

- Milk and milk powder

\section{Confounding, Study Limitations, and} Summary of Findings

- Start of follow up and exposure did not coincide and a potentially important amount of follow-up time is missing from analyses

- Proportions of missing participants differed substantially across exposure groups and was not accounted for in the analyses

- No pre-registered data analysis plan

- High risk of selective reporting from among multiple analyses

\section{Summary:}

Among Chinese pregnant women with healthy $\mathrm{BMI}$, the dietary pattern richer in fruits was positively associated with total GWG, GWG rate, and an increased risk for excessive GWG.

The richer in fish, beans, nuts and yogurt pattern was positively associated with GWG rate in the second trimester and related to a reduced risk for inadequate GWG. 


\section{Study and Participant}

Characteristics $^{\mathrm{xi}}$
Results

Confounding, Study Limitations, and

Summary of Findings

- Cereals: Ref

- Vegetables:

- Meats:

- Fish, beans, nuts, and yogurt: $\beta=0.009,95 \% \mathrm{Cl}$ :

(0.000, 0.018), $P=0.060$

- Milk and milk powder

Second Trimester GWG rate (kg/wk), $\mathrm{P}=\mathrm{NS}$

- Cereals: Ref, $n=821$

- Vegetables: $\mathrm{n}=682$

- Meats: $n=493$

- Fruits: $n=968$

- Milk and milk powder: $\mathrm{n}=645$

Third Trimester GWG rate (kg/wk) , P=NS

- Cereals: Ref, $n=830$

- Vegetables: $n=708$

- Meats: $n=517$

- Fruits: $n=993$

- Fish, beans, nuts, and yogurt: $n=679$

- Milk and milk powder: $\mathrm{n}=669$

GWG adequacy (Ref: Adequate GWG, Cereals DP)

, $\mathrm{P}=\mathrm{NS}$

- Inadequate

- Excessive 


\section{Study and Participant}

Characteristics $^{\mathrm{xi}}$

Wei, 2019 continued
Intervention/Exposure and Outcomes
Results

Significant

GWG (kg), $P<0.01$

- Cereals: $14.5 \pm 4.3$

- Vegetables: $14.5 \pm 4.4$

- Meats: $14.3 \pm 4.3$

- Fruits: $15.1 \pm 4.4$

- Fish, beans, nuts, and yogurt: $14.7 \pm 4.1$

- Milk and milk powder: 14.6 $\pm 4.4$

GWG rate $(\mathrm{kg} / \mathrm{wk}), P=0.01$

- Cereals: $0.37 \pm 0.11$

- Vegetables: $0.37 \pm 0.1$

- Meats: $0.39 \pm 0.11$

- Fruits: $0.38 \pm 0.10$

- Fish, beans, nuts, and yogurt: $0.38 \pm 0.11$

- Milk and milk powder: $0.37 \pm 0.11$

GWG Adequacy, $\mathrm{P}<0.01$

- Cereals:

o Inadequate: $23.5 \%$

- Adequate: $47.6 \%$

o Excessive: $28.9 \%$

- Vegetables:

- Inadequate: $23.6 \%$

o Adequate: $44.9 \%$

o Excessive: $31.5 \%$

- Meats:

o Inadequate: $22.4 \%$
Confounding, Study Limitations, and Summary of Findings

Key confounders accounted for:

None

\section{Summary:}

Among Chinese pregnant women with healthy $\mathrm{BMI}$, the dietary pattern richer in fruits was positively associated with total GWG, GWG rate, and an increased risk for excessive GWG. The richer in fish, beans, nuts and yogurt pattern was positively associated with GWG rate in the second trimester and related to a reduced risk for inadequate GWG. 


\section{Study and Participant}

Characteristics $^{\mathbf{x}}$
Intervention/Exposure and Outcomes

Results

o Adequate: $49.9 \%$

o Excessive: $27.6 \%$

- Fruits:

o Inadequate: $19.2 \%$

- Adequate: $43.6 \%$

o Excessive: $37.2 \%$

- Fish, beans, nuts, and yogurt:

o Inadequate: $19.6 \%$

o Adequate: $48.9 \%$

o Excessive: $31.5 \%$

- Milk and milk powder:

o Inadequate: $20.2 \%$

○ Adequate: $46.9 \%$

o Excessive: $32.9 \%$
Confounding, Study Limitations, and Summary of Findings

\section{Wesolowska, 201917; Poland}

\section{PCS}

Baseline $\mathrm{N}=1306$ Analytic $\mathrm{N}=1158$

(Attrition: 34\%)

$\mathrm{N}=1306$ for participant characteristics

- Age: 17-30y: 65.2\%, >30y: 34.8\%

- Race/Ethnicity: NR

- SES:

o Marital Status: Married: $~ 80.0 \%$

o Education:

- $\leq 9 \mathrm{y}: 2.3 \%$

- 10-12y: $27.9 \%$

- >12y: $69.8 \%$

oSES:

\section{Dietary Pattern(s):}

- Western: High intake of refined grains, processed meat, potatoes, and low intake of whole grains

- Mixed: Intakes in between Western and Prudent

- Prudent: High consumption of fruits, vegetables, legumes, whole grains, poultry, and low-fat and high-fat dairy products

at 20-24wk gestation

\section{Dietary assessment methods:}

Modified version of validated FFQ administered by trained personnel at 2024 wks gestation. Food items grouped into 14

\section{Significant:}

\section{Non-significant:}

GWG Adequacy (Pearson's chi squared test across all GWG and diet categories), $\mathrm{P}=\mathrm{NS}$

\section{Key confounders accounted for:}

None

\section{Limitations:}

- Selection into the study related to exposure and outcome

- Some missing outcome data, reasons NR

- Unclear if outcome was self-reported or assessed objectively. Start of follow up and exposure did not coincide and a potentially important amount of follow-up time was missing from analyses

- Important co-exposures not balanced across groups that were likely to impact the outcome, 


\section{Study and Participant}

Characteristics ${ }^{\mathrm{xi}}$

\section{- Low: 7.4\%}

- Middle: $\sim 67.9 \%$

- High: $24.7 \%$

- Physical Activity: Yes: $68.8 \%$

- Anthropometry: Prepregnancy BMI:

o <18.5: 8.9\%

○ 18.5-24.99: 72.6\%

o $\geq 25: \sim 18.6 \%$

- Smoking status: Cotinine level >10 $\mathrm{ng} / \mathrm{mL}: \sim 11.1 \%$

- Parity:

o 0: $52.0 \%$

$\circ \geq 1: \sim 48.0 \%$

- Alcohol consumption: $~ 6.4 \%$

- Perceived Stress Scale (0-38 pts):

$\geq 17$ points (higher stress): $\sim 52.6 \%$

\section{Intervention/Exposure and Outcomes Results}

predefined food groups: Refined grains,

Whole grains, Low-fat dairy, High-fat dairy,

Butter, Red meat, Poultry, Processed meat,

Fish/Seafood, Fruits, Vegetables, Potatoes,

Legumes, Sweets. 3 dietary patterns derived using exploratory factor analysis.

\section{Outcome \& assessment methods:}

GWG calculated as difference between latest weight before delivery and prepregnancy weight. GWG adequacy determined by IOM 2009 recommendations.
Confounding, Study Limitations, and Summary of Findings

and no adjustment techniques were used to

correct for the issues

- No information on how weight was measured

- No pre-registered data analysis plan

\section{Summary:}

No association was found between GWG and Western, Mixed, or Prudent dietary patterns during pregnancy.

\section{Index/Score}

\section{Ancira-Moreno, 2019 ${ }^{18}$; Mexico PCS, PRINCESA}

Baseline $\mathrm{N}=660$ Analytic $\mathrm{N}=660$

(Attrition: 0\%)

- Age: 24.9y

- Race/Ethnicity: NR

- SES:

○ Married/partnered: 73.9\%

o >9y education: $~ 14.2 \%$

- Anthropometry: Prepregnancy BMI: $\sim 25.7$

- GDM: 0\%

- Gestational HTN: 0\%

- Parity:

o Nulliparous: $48.0 \%$

\section{Dietary Pattern(s)}

Maternal Diet Quality Score (MDQS) based on Mexican Dietary Guidelines and international recommendations for specific foods and nutrients.

- Low adherence (Score 0-2)

- Medium adherence (Score 3-4)

- High adherence (Score $\geq 5$ )

Higher adherence characterized by:

- PUFAS $>6 \%$ of energy intake

- Added sugars $<10 \%$ of energy intake

- Fruits and vegetables $>400 \mathrm{~g} / \mathrm{d}$

- Red meat $<500 \mathrm{~g} / \mathrm{wk}$

- 2 servings/d low fat dairy products

- 2 servings/d legumes

- Foods high in SFAs or added sugar $<10 \%$ of energy intake

\section{Significant:}

GWG rate (kg/wk)

- Middle pregnancy ( $\geq 20$ and <30wk)

o Low: Ref

o Medium: $\beta=-0.0266,95 \%$ Cl: (-0.0496, -0.0037), $\mathrm{P}=0.023$

- Late pregnancy (30-40wk)

o Low: Ref

o Medium: $\beta=0.0256,95 \%$ Cl: $(0.0077,0.0436)$, $\mathrm{P}=0.005$

o High: $\beta=0.0472,95 \% \mathrm{Cl}$ $(0.0222,0.0723), P<0.001$

\section{Key confounders accounted for:}

Age, SES, PA, Prepregnancy BMI, GDM, HTN, Parity

\section{Limitations:}

- Accounted for total energy intake

- Lack of weight measurement at each visit

- Recall bias and other biases related to a priori DP; could not capture day-to-day variability in dietary intake

- Participants who developed HTN or GDM excluded from final analysis

- Selection into analysis related to exposure and outcome and not adjusted for

- Start of follow up and exposure do not coincide and a potentially important amount of follow-up time missing from analyses 


\section{Study and Participant}

Characteristics $^{\mathbf{x i}}$

० 1-2: $28.3 \%$

$\circ \geq 3: \sim 23.3 \%$

- Term birth: $~ 88.0 \%$

\section{Intervention/Exposure and Outcomes}

early-mid pregnancy (0-20 wk), middle

pregnancy ( $\geq 20$ and $<30 \mathrm{wk}$ ), late pregnancy

(30-40wk), and prolonged pregnancy ( $\geq 40 \mathrm{wk}$ )

\section{Dietary assessment methods:}

Dietitian collected data on maternal diet via a multiple-step 24-h dietary recall at each prenatal visit.

Value of 1 assigned if recommendation met and 0 if recommendation not met. Values summed with maximum score of 7 and minimum score of 0 . Three categories of adherence defined: low (0-2 points), medium (3-4 points), and high ( $\geq 5$ points).

\section{Outcome \& assessment methods:}

Weight measured at first and consecutive visits by trained staff using standardized methods. Prepregnancy weight self-reported. Rate of GWG (kg/wk) calculated as weight at current visit minus weight from previous visit, divided by follow-up duration. First GWG rate estimated using prepregnancy weight. Adequacy of GWG rate based on IOM (2009) recommendations.
Results

- Prolonged pregnancy ( $\geq 40 w k$ )

- Low: Ref

- High: $\beta=-0.182,95 \% \mathrm{Cl}$ : $(-$ $0.360,-0.00450), P=0.044$

GWG rate adequacy

\section{- Inadequate}

- Low: Ref

o Medium: OR $=0.742,95 \%$ Cl: $(0.555,0.991)$, $\mathrm{P}=0.044$

○ High: $\mathrm{OR}=0.630,95 \% \mathrm{Cl}$ : $(0.417,0.953), P=0.031$

- Excessive

o Low: Ref

o High: OR=0.623, 95\% Cl: $(0.411,0.942), P=0.025$

GWG rate adequacy,

Prepregnancy $\mathrm{BMI}<25$

- Inadequate

○ Low: Ref

○ Medium: OR=0.426, 95\% Cl: $(0.184,0.985)$, $\mathrm{P}=0.046$

o High: OR=0.295, 95\% Cl: (0.092, 0.946), $\mathrm{P}=0.040$

- Excessive

o Low: Ref

o Medium: OR=0.347, 95\%

Cl: $(0.126,0.952)$,

$\mathrm{P}=0.040$

\section{Confounding, Study Limitations, and} Summary of Findings

- Multiple 24HR recalls collected, but unclear if analysis was cross-sectional

- No pre-registered data analysis plan

- High risk of selective reporting from among multiple analyses

- Baseline differences in maternal age and education

\section{Summary:}

Higher adherence to maternal dietary quality recommendations was protective against inadequate and excessive GWG throughout pregnancy, associated with slower GWG in middle and prolonged late pregnancy, and associated with a faster GWG in early and late pregnancy. 


\section{Study and Participant}

Characteristics ${ }^{\mathrm{xi}}$
Confounding, Study Limitations, and

Summary of Findings o High: OR=0.242, 95\% Cl:

$(0.059,0.989), P=0.048$

GWG rate adequacy,

Prepregnancy BMI $\geq 25$

- Inadequate

o Low: Ref

○ Medium: OR=0.095, 95\%

Cl: $(0.010,0.906)$,

$\mathrm{P}=0.044$

o High: OR= $0.033,95 \% \mathrm{Cl}$ :

(0.002, 0.442), $\mathrm{P}=0.010$

- Excessive

o Low: Ref

- Medium: $\mathrm{OR}=0.084,95 \%$

Cl: $(0.126,0.918)$,

$\mathrm{P}=0.042$

o High: $O R=0.077,95 \% \mathrm{Cl}$ :

$(0.059,0.999), P=0.05$

\section{Non-significant:}

GWG rate (kg/wk)

- Early-mid pregnancy (0-

20wk)

o Low: Ref

o Medium: $\beta=0.0162,95 \%$

$\mathrm{Cl}:(-0.0005,0.0333)$

$\mathrm{P}=0.058$

$\circ$ High, $\mathrm{P}=\mathrm{NS}$

- Middle pregnancy ( $\geq 20$ and $<30 w k$ ) 


\section{Study and Participant}

Characteristics ${ }^{\mathrm{xi}}$

Intervention/Exposure and Outcomes

Results

oHigh: $\beta=-0.0363,95 \%$ CI:

$(-0.076,0.0037), P=0.076$

- Prolonged pregnancy

( $\geq 40 w k$ )

o Low: Re

o Medium, $\mathrm{P}=\mathrm{NS}$

GWG rate adequacy:

- Excessive

o Low: Ref

o Medium: OR=0.773, 95\%

Cl: $(0.572,1.044)$,

$\mathrm{P}=0.094$

\section{Emond, 2018 ${ }^{13}$; U.S.}

\section{PCS, NHBCS}

Baseline $\mathrm{N}=1140$ Analytic $\mathrm{N}=862$

Participants excluded due to unrealistic/missing dietary data $(n=64)$, missing key covariates $(n=89)$, underweight prepregnancy BMI $(n=20)$, incomplete/ unrealistic infant birth data $(n=62)$, or missing urinary arsenic data $(n=43)$

\section{$\mathrm{N}=862$ for all participant} characteristics

- Age: $31.2 y$

- Race/Ethnicity: White, non-Hispanic: $96.8 \%$

- SES:

High school graduate or less: $26.8 \%$

\section{Dietary Pattern(s)}

Adherence to AHEI-2010 diet, by quartile

- Q1: $\mathrm{n}=215$

- Q2: $n=215$

- Q3: $n=216$

- Q4: Highest intake of fruits, vegetables, whole grains, nuts and legumes, long-chain n-3 FAs, and PUFAs; Lowest intake of sugar-sweetened beverages and fruit juice, red and processed meats, trans fatty acids, and sodium $(\mathrm{n}=216)$

at 24-28wk gestation

\section{Dietary assessment methods:}

Women completed a validated FFQ at 2428wk gestation), reflecting usual dietary intake during pregnancy.

Diet quality assessed as adherence to a modified AHEI-2010 for pregnant women, including 10 dietary components: 6 healthful
Confounding, Study Limitations, and

Summary of Findings

\section{Significant:}

\section{Non-significant:}

- $\mathrm{GWG}$ Adequacy, $\mathrm{P}=\mathrm{NS}$

o Insufficient GWG

- Adequate GWG

o Excessive GWG

\section{Key confounders accounted for:}

None

\section{Limitations:}

- Power analysis NR for this secondary analysis

- Selection into study may have been related to exposure and outcome, without adjustment

- Self-reported exposure \& prepregnancy BMI

- Unclear sample size at enrollment

- No preregistered data analysis plan

- Baseline imbalances in multiple key confounders

- No correction for multiple comparisons

\section{Summary:}

Adherence to the AHEI-2010 diet at 24-28wk gestation was not associated with GWG. 


\section{Study and Participant}

Characteristics ${ }^{\mathrm{xi}}$

o Some college: $24.1 \%$

- College graduate or more: $49.1 \%$

- Physical Activity: Regular exercise during pregnancy: $60.6 \%$

- Anthropometry: Prepregnancy BMI:

o 18.5-24.9: $54.5 \%$

○ 25.0-29.9: $26.3 \%$

o $\geq 30: 19.3 \%$

- Smoking status: Smoker: $5.5 \%$

- GDM: $7.1 \%$

o History of DM: $5.9 \%$

- Gestational HTN: PE: 6.7\%

o History of HTN: $1.9 \%$

- Parity: Nulliparous: $41.0 \%$

\section{Intervention/Exposure and Outcomes}

Results

Confounding, Study Limitations, and

Summary of Findings components to encourage (vegetables, fruits,

whole grains, nuts/legumes, long-chain n-3

FAs from foods and supplements,

polyunsaturated fats) and 4 components to

reduce (sugary beverages, red and

processed meats, trans fatty acids, and

sodium). All components are scored from 0 to

10 for a total score of $0-100$ such that a

higher score indicates a healthier intake.

\section{Outcome \& assessment methods:}

GWG computed using self-reported

prepregnancy weight and the last recorded prenatal weight from prenatal medical records. Adequacy of GWG defined

according to IOM 2009 recommendations.

\section{Fernández-Barrés, 2019²; Spain PCS, INMA}

Baseline $\mathrm{N}=2195$ Analytic $\mathrm{N}=2127$

Participants excluded from analysis if no dietary data at 3rd trimester $(\mathrm{n}=$ $319)$ or no BMI measured $(n=248)$; Those lost to follow-up were younger smoked more, and had lower socioeconomic and education levels.

- Age: $30.9 y$

- Race/Ethnicity: NR

- SES:

Social class:

- I+II: 23.2\%

\section{Dietary Pattern(s)}

- Adherence to Relative Mediterranean Diet (rMED) score: consumption of vegetables, fruits and nuts, cereals, legumes, fish, olive oil, meat, and dairy products. Alcohol not scored.

- T1: low rMED (Score 0-7), $\mathrm{n}=925$

- T2: medium rMED (Score 8-9), $\mathrm{n}=631$

- T3: high rMED (Score 10-15), n=639

- *highest score differs btw 15 and 16 throughout the paper

at $12 \mathrm{wk}$ and $32 \mathrm{wk}$ gestation

\section{Dietary assessment methods:}

101-item validated FFQ at 12 and 32wk gestation measured diet in the first trimester

\section{Significant:}

GWG (kg/wk), P=0.017

- T1: $0.35 \pm 0.13$

- T2: $0.35 \pm 0.12$

- T3: $0.34 \pm 0.13$

\section{Non-significant:}

\section{Key confounders accounted for:}

None

\section{Limitations:}

- Start of follow up and exposure did not coincide and potentially important amount of follow-up time was missing from analyses

- Important co-exposures were not balanced across groups that were likely to impact the outcome, and no adjustment techniques were used to correct for the issues

- No pre-registered data analysis plan

\section{Summary:}




\section{Study and Participant}

Characteristics $^{\mathrm{xi}}$

- III: $27.1 \%$

- IV+V: $49.7 \%$

o Education level:

- Primary or less: $22.1 \%$

- Secondary: $41.5 \%$

- University: 36.4\%

- Physical Activity: METs(hr/d): 37.4

- Anthropometry: Prepregnancy BMI: $\sim 23.5$

- Smoking status: During pregnancy: $\sim 16.6 \%$

- GDM: $4.1 \%$

o History of GDM: 0.3\%

- Parity:

o Primiparous: $~ 57.0 \%$

o Multiparous: $\sim 43.0 \%$

\section{Intervention/Exposure and Outcomes}

Results

and 12-32wk. Adherence to the

Mediterranean diet assessed using the rMED.

Values of 0,1 , and 2 assigned to intake tertiles, positively scoring higher intakes for the 6 components that fit into the

Mediterranean diet (vegetables, fruits and

nuts, cereals, legumes, fish, olive oil). Scoring

reversed for meat and dairy. Scores summed for each component, for a total score ranging from 0 to 16 .

\section{Outcome \& assessment methods:}

GWG extracted from prenatal visit records; timing NR
Confounding, Study Limitations, and Summary of Findings

Higher adherence to the Mediterranean diet during pregnancy is associated with lower GWG.

\section{Fulay, 2018'2; U.S.}

PCS, Project Viva

Baseline $\mathrm{N}=2128$ Analytic $\mathrm{N}=1756$

(Attrition: 17\%)

Participants excluded due to Type 1 or 2 Diabetes Mellitus $(n=16)$, missing outcomes data $(n=7)$ or dietary data $(n=345)$

$\mathrm{n}=\sim 1760$ for all participant characteristics
- Age:
o 15-24y: $7.4 \%$
○ 25-34y: $63.9 \%$
० 35-44y: $28.8 \%$
- Race/Ethnicity:

\section{Dietary Pattern(s)}

- Adherence to DASH diet

- Higher adherence characterized by higher intakes of fruits, vegetables, whole grains, nuts/legumes, and low-fat dairy, and lower intakes of sodium, sugar-sweetened beverages, and red and/or processed meats.

- Adherence to DASH OMNI diet at 11 wk gestation.

- Higher adherence characterized by higher intakes of fruits, vegetables, whole grains, nuts/legumes, low-fat dairy, and MUFAs and PUFAs, and lower intakes of sodium, sugar-sweetened beverages, and red and/or processed meats.

at $11 \mathrm{wk}$ gestation

\section{Significant:}

Subsequent GWG (kg) per unit 1st trimester DASH score

- Prepregnancy obese

$$
(n=244)
$$

$\circ \beta=0.31,95 \% \mathrm{Cl}: 0.08,0.53$

Subsequent GWG $(\mathrm{kg})$ per unit 1st trimester DASH OMNI score

\footnotetext{
- Prepregnancy obese $(\mathrm{n}=244)$

$\circ \beta=0.34,95 \% \mathrm{Cl}:(0.09$, $0.58)$
}

\section{Non-significant}

\section{Key confounders accounted for:}

Age, Race/ethnicity, SES, Pre-preg BMI, Smoking, Parity

\section{Limitations:}

- Accounted for total energy intake

- Power analysis NR for this exploratory study based on secondary analysis of existing data

- Adjusted for potential mediator (early GWG)

- Start of follow up and start of exposure did not coincide

- Unclear proportions/reasons for missing exposure data

- Self-reported diet and prepregnancy weight

- No pre-registered data analysis plan

Summary: 


\section{Study and Participant}

Characteristics ${ }^{\mathrm{xi}}$

\section{o Black: $12.3 \%$}

o Hispanic: $6.5 \%$

o White: $72 \%$

- Asian: $5.6 \%$

o Other: $3.6 \%$

\section{- SES:}

- Annual household income

- <\$20,000: $2.9 \%$

- > \$70,000: $59.6 \%$

- Married/cohabiting: $93.3 \%$

- Education

- Primary: $9.4 \%$

- Secondary: $58.6 \%$

- $\geq$ College: $32.0 \%$

- Anthropometry: Prepregnancy BMI: o <18.5: $3.9 \%$

- 18.5-24.9: $60.7 \%$

○ 25.0-29.9: $21.4 \%$

o $\geq 30: 12.7 \%$

- Smoking status: During pregnancy: $10.9 \%$

- GDM: $5.2 \%$

- Gestational HTN: History before pregnancy: $4.5 \%$

- Parity:

○ $0: 49.3 \%$

$\circ 1: 35.3 \%$

$\circ \geq 2: 15.4 \%$

\section{Intervention/Exposure and Outcomes}

\section{Dietary assessment methods:}

Semi-quantitative, validated, 140-item FFQ based on NHS, administered at enrollment (11.1wk gestation).DASH score calculated as weighted sum of frequency of intake/day for the following components, with increasingly postitive scores for higher quintiles of intake for fruits, vegetables, whole grains,

nuts/legumes, and low-fat dairy, and for lowe quintiles of intake for sodium, sugarsweetened beverages, and red and/or processed meats. Total score range: 840.The DASH diet is rich in fruits, vegetables, legumes, whole grains, and healthy fats, with limited amounts of poultry, red meat, and dairy. The DASH diet also focuses on intake of foods high in macro- and micronutrients that have been specifically demonstrated to be effective in reducing risk of hypertension: reduced amounts of saturated fat, total fat, and cholesterol; and high levels of potassium, magnesium, calcium, fiber, and protein, with $\leq 3 \mathrm{~g} / \mathrm{d}$ sodium.

DASH OMNI score calculated similarly to DASH score, with the additional component of increasingly postitive scores for higher quintiles of intake of MUFAs and PUFAs. Total score range: 9-45.

\section{Outcome \& assessment methods:}

GWG from time of FFQ in first trimester to delivery. Derived via linear interpolation between the two measures of weight assessed in closest temporal proximity to the date of FFQ administration and calculated the
Results

Subsequent GWG (kg) per unit 1st trimester DASH score, $\mathrm{P}=\mathrm{NS}$

- Prepregnancy underweight $(n=68)$

- Prepregnancy normal weight $(n=1068)$

- Prepregnancy overweight $(n=376)$

Subsequent GWG $(\mathrm{kg})$ per unit 1 st trimester DASH OMNI score, $\mathrm{P}=\mathrm{NS}$

- Prepregnancy underweight $(n=68)$

- Prepregnancy normal weight $(n=1068)$

- Prepregnancy overweight $(n=376)$

\section{Confounding, Study Limitations, and} Summary of Findings

Among women with prepregnancy obesity, higher adherence to a DASH or DASH OMNI diet is associated with higher GWG from 1st trimester through delivery. This association was not statistically significant among women with prepregnancy $\mathrm{BMI}<30$. 


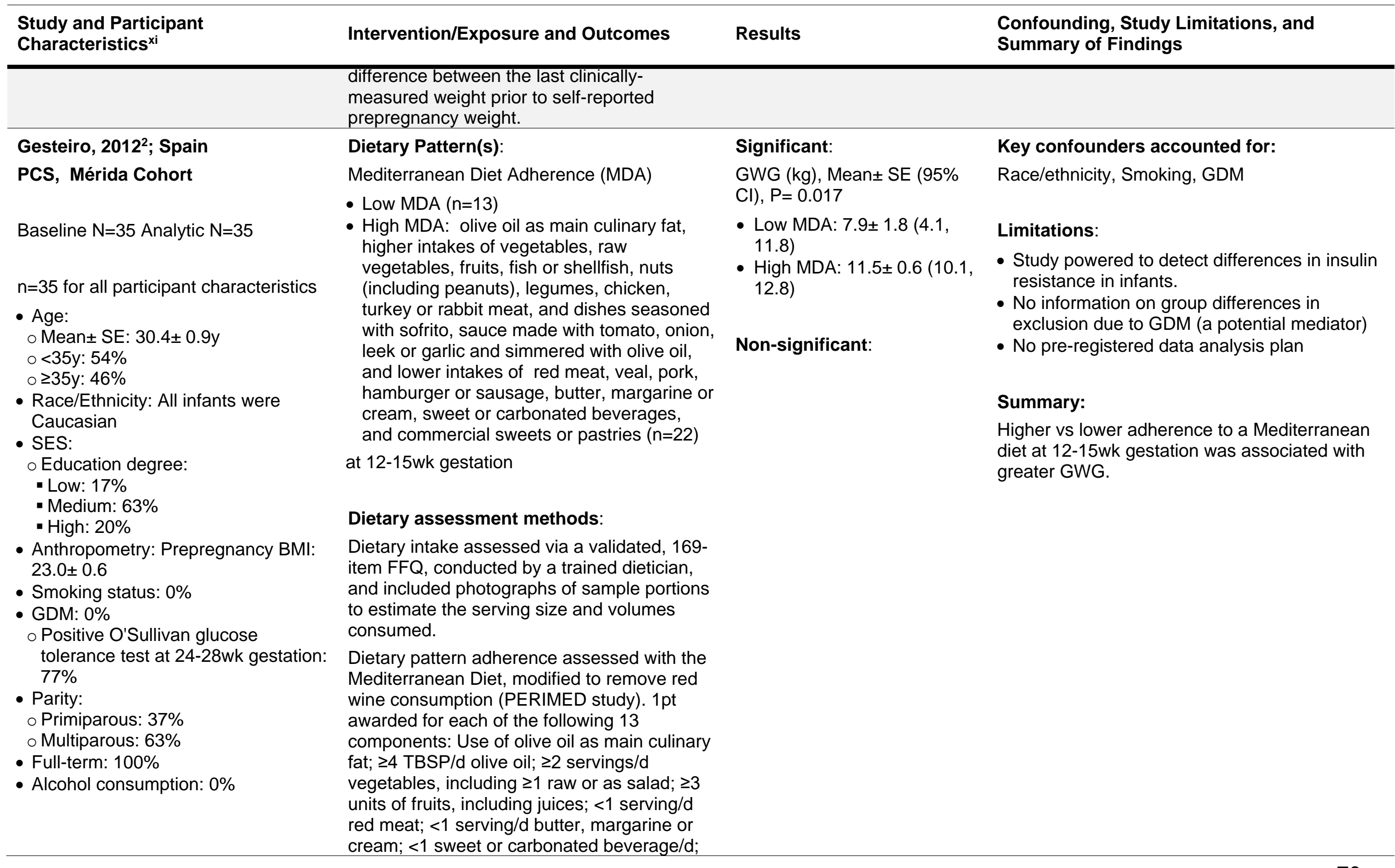




\section{Study and Participant}

Characteristics ${ }^{\mathrm{xi}}$
Intervention/Exposure and Outcomes

Results

Confounding, Study Limitations, and

Summary of Findings

$\geq 3$ servings/wk legumes; $\geq 3$ servings/wk fish

or shellfish; $<3$ commercial (not home-made)

sweets or pastries; $\geq 3$ servings/wk nuts

(including peanuts); Consumption of chicken,

turkey or rabbit meat instead of veal, pork,

hamburger or sausage; 2 servings/wk

vegetables, pasta, rice or dishes seasoned

with sofrito, sauce made with tomato, onion,

leek or garlic and simmered with olive oil

Low Mediterranean Diet adherence (MDA)

defined as score $<7$, and high MDA defined

as scores $\geq 7$.

\section{Outcome \& assessment methods:}

Anthropometrical measurements were taken

by trained personnel following hospital's

standard procedures. GWG data obtained

from hospital records

Gesteiro, 2012 continued

\section{Dietary Pattern(s):}

Adherence to Healthy Eating Index

- Inadequate HEI $(n=19)$

- Adequate HEI $(n=16)$

- Perfect HEI adherence encourages 6-10 servings/d Cereals, grains, and legumes; 3 -

5 servings/d Vegetables; $2-4$ servings/d

Fruits; $2-3$ serving/d Milk and dairy

products; $2-3$ servings/d Meat, eggs and

fish; $\leq 30 \%$ FAT; $\leq 10 \%$ SFA; $<300 \mathrm{mg} / \mathrm{d}$

Cholesterol; <2,400 mg/d Sodium; 16

different food items diet variety over $3 d$

at $12-15$ wk gestation

\section{Significant:}

\section{Non-significant}

GWG (kg), Mean, $P=N S$

\section{Key confounders accounted for:}

Race/ethnicity, Smoking, GDM

\section{Summary:}

Adequate vs inadequate adherence to a Healthy Eating Index was not associated with differential GWG.

\section{Dietary assessment methods}




\section{Study and Participant}

Dietary pattern assessed with the Healthy

Eating Index (HEI), modified for the Spanish population (Kennedy et al), based on a 10-

component, 100-point scale, taking into account recommended energy intakes of for 1600,2200 and $2800 \mathrm{kcal}$ and the required servings. Diets with $\mathrm{HEl}$ scores of $\leq 70$ were labelled 'inadequate', vs 'adequate' (scores $>70$ )

Dietary components included 10 groups (by 1600,2200 and $2800 \mathrm{kcal} / \mathrm{d}$

recommendations): Cereals, grains, and

legumes $(0=0$ servings, $10=6,8,10$ servings) Vegetables $(0=0$ servings, $10=3,4,5$ servings); Fruits ( $0=0$ servings, $10=2,3,4$ servings); Milk and dairy products $(0=0$ servings, $10=2-3$ servings) ; Meat, eggs and fish ( $0=0$ servings, $10=2-3$ servings); $\%$ FAT $(0=\geq 45 \%, 10=\leq 30 \%) ; \%$ SFA $(0=\geq 15 \%$,

$10=\leq 10 \%)$; Cholesterol $(0=\geq 450 \mathrm{mg} / \mathrm{d}, 10=$ $<300 \mathrm{mg} / \mathrm{d})$; Sodium $(0=\geq 4,800 \mathrm{mg} / \mathrm{d}$,

$10=<2,400 \mathrm{mg} / \mathrm{d})$; Diet variety over $3 \mathrm{~d}(0=\leq 6$ different food items, $10=16$ different food items)

\section{Hillesund, 2018 ${ }^{21}$; Norway}

\section{PCS, NFFD}

Baseline $\mathrm{N}=606$ Analytic $\mathrm{N}=524$ Twelve women withdrew due to miscarriage $(n=6)$, twin pregnancy $(n=2)$, and moving away $(n=4)$, very low BMI $(n=1)$, and personal reasons $(n=2)$, leaving 591 eligible for the present analyses.

\section{Dietary Pattern(s):}

- Adherence to the Norwegian Fit for Delivery (NFFD) diet,

- Low (Score 0-3), $n=151$

- Medium (Score 4-5), $n=204$

- High (Score 6-10), n=236

- Higher scores characterized by eating/drinking: $\geq 24$ main meals/wk, water for $\geq 44 \%$ of drinking events, vegetables with dinner $\geq 5 x / w k$, fruits or vegetables as snacks $\geq 3 \mathrm{x} / \mathrm{wk},<1 \mathrm{x} / \mathrm{d}$ sugar-rich food items, $<1 \mathrm{x} / \mathrm{d}$ fast-foods, snacks, or other

\section{Significant:}

GWG Adequacy, per 1pt NFFD score

- Excessive (Ref: Optimal GWG), $n=528, P=0.024$ ○ $\mathrm{aOR}=0.90,95 \% \mathrm{Cl}:(0.83$, 0.99)

\section{Subanalysis additionally} adjusting for PA

\section{Key confounders accounted for:}

Age, SES, PA (subanalyses), Pre-preg BMI, Smoking, Parity

\section{Limitations:}

- Models adjusted for randomization assignment, but residual confounding could remain

- The study was restricted to nulliparous women, so the findings may not be generalizable 


\section{Study and Participant}

Characteristics $^{\mathrm{xi}}$

$\mathrm{n}=\sim 591$ for participant characteristics

- Age: $28.0 \pm 4.4$ y (range 18-44y) ० <25y: $25.2 \%$,

० $25-29 y: 46.2 \%$

○ $30-34 \mathrm{y}: 21.8 \%$

o $\geq 35 \mathrm{y}: 6.8 \%$

- Race/Ethnicity: "predominantly

White, European"

- SES: Education:

o $\leq 12 \mathrm{y}: 31.8 \%$

o $13-15 y: 32.7 \%$

o $\geq 16 y: 35.5 \%$

- Occupation:

- Work outside home: $84.2 \%$

○ Student: $8.7 \%$

o Unempoyed: $3.9 \%$

o Sick leave/disabled: $1.9 \%$

o Homemaker: $1.4 \%$

- Income (NOK):

o $\leq 400,000: 31.2 \%$

o >700,000: $34.4 \%$

o Refrain from response: $6.6 \%$

- Marital Status:

○ Married/boyfriend/partner: $96.2 \%$

- PA: The intervention also included supervised exercise classes (2/wk), including strength training and cardiovascular exercise at moderate intensity

- PA level in early pregnancy:

o Low activity: $26.4 \%$

- Medium activity: $58.2 \%$

- High activity: $15.4 \%$

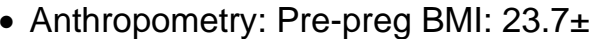
3.9

$0<19: 0 \%$

\section{Intervention/Exposure and Outcomes}

salty food, never eating sweets and snacks without appreciation, buying small portion size of $\geq 1$ unhealthy food items, eating beyond satiety $<1 / \mathrm{wk}$, and reading nutrition labels on foods sometimes or often.

at $15 \mathrm{wk}$ gestation

\section{Dietary assessment methods:}

43-item FFQ, including questions about selected aspects of current diet and dietary behavior, mainly those targeted in NFFD.

The subscales could be single variables or sum scores constructed from relevant questionnaire responses. Each subscale was dichotomized with the sample median as cutoff, and participants with the healthier behavior were assigned ' 1 ' in each subscale, whereas the other half of the sample was assigned ' 0 '. Individual diet scoring ranged from 0 to 10 , with higher score indicating healthier behavior.

\section{Outcome \& assessment methods:}

GWG calculated as final measured weight within $2 w k$ of delivery among women at term ( $\geq 37$ wk gestation) minus self-reported prepregnancy weight. GWG adequacy defined by 2009 IOM recommendations.
Results

GWG Adequacy, per 1pt

NFFD score

- Excessive (Ref: Optima GWG, Low adherence), $\mathrm{n}=418, \mathrm{P}=0.009$

- $\mathrm{aOR}=0.88,95 \% \mathrm{Cl}:(0.79$ 0.97)

\section{Non-significant}

GWG Adequacy, per 1pt NFFD score

- Inadequate (Ref: Optimal GWG, Low adherence), $\mathrm{n}=524, \mathrm{P}=\mathrm{NS}$

Sub-analysis additionally adjusting for PA

GWG Adequacy, per 1pt NFFD score

Inadequate (Ref: Optimal GWG, Low adherence), $(n=414), P=N S$

\section{Confounding, Study Limitations, and} Summary of Findings

- Diet score showed test-retest reproducibility but this was not validated against other methods of operationalizing dietary behavior

- Not all participants who would have been eligible for the target trial were included in the study

- Single FFQ did not address full diet

- Smoking status varied by diet score, but included in models

- May not have accounted for all effects of coexposures across groups

- Total missingness similar across groups, but unclear proportions for different reasons for missingness

- The outcome measure may be influenced by knowledge of the exposure received by study participants

- No pre-registered data analysis plan

\section{Summary:}

Higher adherence to NFFD diet at $15 \mathrm{wk}$ gestation is associated with lower odds of excessive GWG at term, regardless of dietary and exercise intervention. 


\section{Study and Participant}

Characteristics $^{\mathrm{xi}}$

$<25: 72.2 \%$

$\circ$ 25-29.9: $20.2 \%$

$\circ \geq 30: 7.6 \%$

- Smoking status: Current: $3.9 \%$

- GDM: 9.1\%

- Gestational HTN: PE: 4.3\%

- Parity: Nulliparous: $100 \%$

\section{Hillesund, 20145; Norway \\ PCS, MoBa}

Baseline $\mathrm{N}=66,597$ Analytic $\mathrm{N}=56629$

(Attrition: 15\%)

- Age: $30.1 \pm 4.6 y$

- Race/Ethnicity: NR

- SES:

- Education

- $\leq 12 \mathrm{y}: ~ 33.3 \%$

-13-16y: 33.3\%

- $\geq 17 \mathrm{y}:-33.3 \%$

- Anthropometry: Prepregnancy BMI: $24.0 \pm 4.2$

- Smoking: During pregnancy: 7.2\%

- Physical Activity: Exercise:

o Rarely: $\sim 37.4 \%$

o $1-2$ times/wk: $~ 32.1 \%$

$\circ \geq 3$ times/wk: $~ 30.5 \%$

- GDM: History of DM: $0 \%$

- Parity:

० 0: $\sim 52.7 \%$

० 1: $30.2 \%$

○ 2: 13.4\%

$\circ \geq 3: \sim 3.7 \%$

\section{Dietary Pattern(s):}

Adherence to New Nordic Diet (NND)

- Low, $\mathrm{n}=17802$

- Medium, $n=23558$

- High, $n=25237$

- Higher scores characterized by eating/drinking: $\geq 24$ main meals/wk, Nordic fruits $\geq 5 / w k$, root vegetables $\geq 5 / w k$, cabbage $\geq 2 / w k$, potatoes at least one-third of total occasions of eating potatoes, rice or pasta, whole grain bread more often than refined bread, oatmeal $\geq 1 / \mathrm{mo}$, fish/game/berries $\sim 2 / \mathrm{wk}$, milk more often than juice, $\geq 6$ times as much water as sugar-sweetened beverages

at 22wk gestation

\section{Dietary assessment methods:}

Self-administered, semi-quantitative FFQ validated for use in MoBa (see Brantsaeter, 2008)

\section{Outcome \& assessment methods:}

GWG, difference between prepregnancy body weight and body weight at birth, as

\section{Significant:}

GWG (kg)

- Overall: $15.0 \pm 5.9$

- Inadequate: $6.8 \pm 3.9$

- Optimal: $12.8 \pm 2.5$

- Excessive: $19.3 \pm 4.7$

GWG adequacy (\%)

- All Women, $\mathrm{P}<0.001$

o Low:

- Inadequate: 18.7

- Optimal: 33.0

- Excessive: 48.3

- Medium:

- Inadequate: 18.4

- Optimal: 34.6

- Excessive: 47.1

o High:

- Inadequate: 18.6

- Optimal: 35.9

- Excessive: 45.4

- Prepregnancy BMI <25, $\mathrm{P}=0.008$

o Low:

- Inadequate: 21.8

- Optimal: 39.1

\section{Key confounders accounted for:}

None

\section{Limitations:}

- Limited generalizability due to homogenous sample

- Potential selection bias because of excluding preterm and post-term infants

- Start of follow up and start of exposure did not coincide

- Self-reported outcome

- Unclear why the sample size in table 4 was smaller than other tables

- Energy intake was potentially on the causal pathway, but was still adjusted for in the multivariable regression model

\section{Summary:}

Greater adherence to the NND during pregnancy may facilitate optimal GWG, especially among with healthy prepregnancy $\mathrm{BMI}$ 


\section{Study and Participant}

Characteristics $^{\mathrm{xi}}$

\section{Intervention/Exposure and Outcomes}

reported in questionnaires at 17wk gestation and 6 mo postpartum.

GWG categorized into inadequate, optimal and excessive (2009 IOM recommendations)

Results

- Excessive: 39.1

o Medium:

- Inadequate: 21.7

- Optimal: 40.2

- Excessive: 38.1

o High:

- Inadequate: 21.8

- Optimal: 41.6

- Excessive: 36.6

\section{Non-significant:}

GWG adequacy (\%)

- Prepregnancy BMI $\geq 25$,

$\mathrm{P}=0.07$

o Low:

- Inadequate: 12.7

- Optimal: 21.4

- Excessive: 65.9

o Medium:

- Inadequate: 10.8

- Optimal: 21.9

- Excessive: 67.2

o High:

- Inadequate: 10.2

- Optimal: 20.8

- Excessive: 69.0

\section{Significant:}

Inadequate GWG (Ref: Optimal GWG, Low NND), aOR $(95 \% \mathrm{Cl})$

- Prepregnancy BMI $\geq 25$

○ Medium: 0.86 (0.74, 0.99), $\mathrm{P}=0.038$
Confounding, Study Limitations, and Summary of Findings

pregnancy may facilitate optimal GWG,




\section{Study and Participant}

Characteristics ${ }^{\mathrm{xi}}$
Intervention/Exposure and Outcomes

Results

Excessive GWG (Ref:

Optimal GWG, Low NND), aOR $(95 \% \mathrm{Cl})$

- Prepregnancy BMI <25

o High: 0.93 (0.87, 0.99), $P=0.024$

\section{Non-significant:}

Inadequate GWG (Ref: Optimal GWG, Low NND), aOR $(95 \% \mathrm{Cl})$

- Prepregnancy BMI <25 - Medium, $\mathrm{P}=\mathrm{NS}$

o High, $\mathrm{P}=\mathrm{NS}$

- Prepregnancy $\mathrm{BMI} \geq 25$

o High, $\mathrm{P}=\mathrm{NS}$

Excessive GWG (Ref: Optimal GWG, Low NND), aOR $(95 \% \mathrm{Cl})$

- Prepregnancy BMI <25

- Medium, $\mathrm{P}=\mathrm{NS}$

- Prepregnancy $\mathrm{BMI} \geq 25$

Medium, $\mathrm{P}=\mathrm{NS}$

\section{Hrolfsdottir, 2019 ${ }^{14}$; Iceland} PCS, PREWICE

Baseline $\mathrm{N}=1326$ Analytic $\mathrm{N}=1326$

$n=1326$ for all participant

characteristics

\section{Dietary Pattern(s):}

- Low risk diet score ( $\leq 2 \mathrm{pt}), \mathrm{n}=305$

- Medium risk diet score (3 pt), $n=632$

- High risk diet score ( $\geq 4 \mathrm{pt}), \mathrm{n}=389$

- Higher risk score (out of 5) characterized by: Not eating a varied diet, $<5 / d$ fruits/vegetable , <2/d dairy, $<2 / d$ whole

\section{Significant:}

Excessive GWG

- Per unit increase in score: aRR: $1.10(1.01,1.19)$

- Per SD increase in score: aRR: $1.08,95 \% \mathrm{Cl}:(1.01$ 1.15), $P<0.05$

\section{Confounding, Study Limitations, and}

Summary of Findings

especially among with healthy prepregnancy

$\mathrm{BMI}$

\author{
-
}




\section{Study and Participant}

Characteristics $^{\mathbf{x i}}$

- Age: $30.2 \pm 5.2 y$

- Race/Ethnicity: NR

- SES:

o Married status: Single: $6 \%$

o Education:

- Elementary: 13\%

- High \& technical school: $29 \%$

- University: 35\%

- Higher academic: 24\%

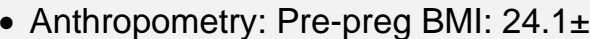
6.5

$0<18.5: 4 \%$

○ 18.5-24.9: $55 \%$

o $\geq 25.0-30.0: 24 \%$

$\circ \geq 30.0: 18 \%$

- Smoking status:

o Before preg: $16 \%$

o During preg: $7 \%$

- GDM: 19.9\%

- Parity: Nulliparous: 39\%

\section{Intervention/Exposure and Outcomes}

grain products, $\geq 5 /$ wk sugar/artificially sweetened beverages, and $\geq 5 / d$ dairy.

at 11-14wk gestation

\section{Dietary assessment methods:}

Risk factors for inadequate diet assessed from 40-item FFQ. Women reported diet in the previous $4 \mathrm{wk}$, corresponding to the first trimester (enrolled in 11-14 week of pregnancy). This information was converted to frequency/wk for all food groups, which was then transformed into 13 predefined dietary risk factors for inadequate diet, based on the Icelandic Food-Based Dietary

Recommendations, which are based on the Nordic Nutrition Recommendations (2014). If the women excluded/avoided any of the main food groups (cereal, vegetables/ fruits, fish, meat, eggs, high-fat foods, or dairy), they were categorized to the group not eating a varied diet.

After logistic regression modeling to predict excessive GWG, the six dietary risk factors (predictors) were included in the final model. To construct a total dietary risk score, each participant got 1 for fulfilling the risk criteria and 0 for not fulfilling the risk criteria. The scores of the six dietary risk factors were then summed up, ranging from scores of 0 to 5 as it was not possible to be in both milk risk groups (too low/too high).

\section{Outcome \& assessment methods:}

Excessive GWG: $>18 \mathrm{~kg}$ in women with normal pre-pregnant weight and $>12 \mathrm{~kg}$ in

\section{Confounding, Study Limitations, and}

Summary of Findings

- Low risk diet score: Cases=99 $(32 \%)$

- High risk diet score: Cases $\mathrm{n}=160(41 \%)$

o aRR vs Low risk scores: $1.23,95 \% \mathrm{Cl}$ : $(1.002$

1.50), $\mathrm{P}<0.05$

\section{Non-significant:}

Excessive GWG

- Low risk diet score: Cases $\mathrm{n}=99(32 \%)$

- Medium risk diet score:

Cases $n=217$ (34\%)

o aRR vs Low scores: 1.04 , $95 \% \mathrm{Cl}:(0.86,1.26)$, $\mathrm{P}=\mathrm{NS}$

Analyses excluding 264 GDM cases:

Excessive GWG

- Per one unit increase in score: aRR: $1.09,95 \% \mathrm{Cl}$ : $(1.00,1.19), \mathrm{P}=\mathrm{NS}$

- Low risk diet score: Cases $\mathrm{n}=89 / 266(34 \%)$

- Medium risk diet score: Cases $n=184 / 533$ (35\%) o aRR vs Low scores: 1.01, 95\% Cl: $(0.82,1.23)$, $\mathrm{P}=\mathrm{NS}$
- Potential differential exposure time between groups

- Unclear whether analyses accounted for group differences in missing data

- Unclear systematic errors in outcome measurement by exposure

- No pre-registered data analyses

\section{Summary:}

A higher dietary risk score, including a nonvaried diet, a nonadequate intake of fruits/vegetables, dairy, and whole grain, as well as an excessive intake of sugar/artificially sweetened beverages and dairy, was associated with a higher risk of excessive GWG, but the association disappeared after removing GDM cases. 


\begin{tabular}{|c|c|c|c|}
\hline $\begin{array}{l}\text { Study and Participant } \\
\text { Characteristics }^{\mathrm{xi}}\end{array}$ & Intervention/Exposure and Outcomes & Results & $\begin{array}{l}\text { Confounding, Study Limitations, and } \\
\text { Summary of Findings }\end{array}$ \\
\hline & $\begin{array}{l}\text { women with pre-pregnant overweight and } \\
\text { obesity } \\
\text { GWG retrieved from the maternal hospital } \\
\text { records as women were weighed in antenatal } \\
\text { visits. Total GWG calculated as the difference } \\
\text { between the highest recorded weight ( } \geq 36 w k) \\
\text { and prepregnancy weight. }\end{array}$ & $\begin{array}{l}\text { - High risk diet score: Cases } \\
\text { n=136/326 (42\%) } \\
\text { o aRR vs Low scores: } 1.19 \\
\text { 95\% Cl: }(0.96,1.47) \\
\text { P=NS }\end{array}$ & \\
\hline Hrolfsdottir, 2019 continued & $\begin{array}{l}\text { Dietary Pattern(s): } \\
\text { - Score based on three dietary risk factors } \\
\text { most strongly associated with excessive } \\
\text { GWG in the multivariable model. Higher } \\
\text { dietary risk scores characterized by } \geq 5 / \text { wk } \\
\text { sugar- and artificially sweetened beverages, } \\
<2 / d \text { whole grain products, and } \geq 5 / d \text { dairy } \\
\text { at } 11-14 \text { wk gestation }\end{array}$ & $\begin{array}{l}\text { Significant: } \\
\text { Excessive GWG } \\
\text { Per SD increase in score } \\
\text { aRR: } 1.08,95 \% \mathrm{Cl}:(1.002, \\
1.15), \mathrm{P}<0.05 \\
\text { Non-significant: }\end{array}$ & $\begin{array}{l}\text { Key confounders accounted for: } \\
\text { Pre-preg BMI, Smoking, GDM (in subanalyses), } \\
\text { Parity } \\
\text { Summary: } \\
\text { A higher dietary risk score, including excessive } \\
\text { intake of sugar- and artificially sweetened } \\
\text { beverages, inadequate intake of whole grains, } \\
\text { and nonadequate intake of dairy was } \\
\text { associated with a higher risk of excessive } \\
\text { GWG. }\end{array}$ \\
\hline Hrolfsdottir, 2019 continued & $\begin{array}{l}\text { Dietary Pattern(s): } \\
\text { Diet score based on } 13 \text { dietary risk factors. } \\
\text { Higher dietary risk scores characterized by: } \\
\text { not eating a varied diet, }<5 / \mathrm{d} \text { vegetables } \\
\text { and fruits, }<2 / \mathrm{d} \text { fish, }<2 / \mathrm{d} \text { dairy, }<2 / \mathrm{d} \text { whole } \\
\text { grain products, }<3.5 / \text { wk beans, nuts, seeds, } \\
<5 / \text { wk } \mathrm{D} \text {-vitamin, using butter rather than oil } \\
(\geq 50 \%), \geq 1 / \text { wk french fries and fried } \\
\text { potatoes, } \geq 2.5 / \text { wk sweets, ice cream, } \\
\text { cakes, cookies, } \geq 5 / \text { wk sugar- and artificially } \\
\text { sweetened beverages, } \geq 5 / \mathrm{d} \text { dairy intake, } \\
\geq 1 / \text { wk processed meat products } \\
\text { at } 11-14 \text { wk gestation }\end{array}$ & $\begin{array}{l}\text { Significant: } \\
\text { Non-significant: } \\
\text { Excessive GWG: } \\
\text { Per SD increase in score, } \\
P=N S\end{array}$ & $\begin{array}{l}\text { Key confounders accounted for: } \\
\text { Pre-preg BMI, Smoking, GDM (in subanalyses), } \\
\text { Parity } \\
\text { Summary: } \\
\text { A higher dietary risk score including } 13 \text { dietary } \\
\text { risk factors (as described in the exposure) was } \\
\text { not associated with the risk of excessive GWG. }\end{array}$ \\
\hline $\begin{array}{l}\text { Meinila, } 2017^{27} \text {; Finland } \\
\text { PCS, Control arm of RADIEL trial }\end{array}$ & $\begin{array}{l}\text { Dietary Pattern(s): } \\
\text { Healthy Food Intake Index (HFII) adherence }\end{array}$ & Significant: & $\begin{array}{l}\text { Key confounders accounted for: } \\
\text { None }\end{array}$ \\
\hline
\end{tabular}




\section{Study and Participant}

Characteristics $^{\mathbf{x}}$

\section{Intervention/Exposure and Outcomes}

- HFII score 0-7 $(n=24)$

Baseline $\mathrm{N}=137$ Analytic $\mathrm{N}=137$

- Age: 32y

- Race/Ethnicity: NR

- SES: Education: 14.3y

- PA: Leisure-time physical activity: $\sim 78.3 \mathrm{~min} / \mathrm{wk}$

- Anthropometry: BMI at 12.0-14.6wk: $\sim 31.7$

- $100 \% \geq 30 \mathrm{BMI}$ or with history of GDM

- GDM: 40.1\%

- Parity: Nulliparous: $37.2 \%$

- HFII score 8-12 $(n=84)$

- HFIl score 13-17 ( $n=29)$

- Higher scores are indicative of higher adherence to the Nordic Nutrition whole-grains, and fish and poultry, and and refined grains.

at first trimester ( 13wk)

\section{Dietary assessment methods:}

Recommendations (NNR), characterized by higher consumption of fruits, vegetables, lower intakes of red and processed meat,

Diet assessed during the first trimester via FFQ. The HFII comprised the following components: snacks, low-fat cheese, fish, low-fat milk, vegetables, fruits and berries, sugar-sweetened beverages, high-fiber grains, fast food, fat spread, and cooking fat.

The HFIl scores were divided into three categories by setting $z$ cut-off limits at \pm 1 SD from the mean.

\section{Outcome \& assessment methods:}

Weight measured by a study nurse. GWG from 12.0-14.6wk through 22.4-24.1wk gestation.

\section{Poon, 20137; U.S.}

PCS, IFPSII

Baseline $\mathrm{N}=893$ Analytic $\mathrm{N}=893$

(Attrition: 0\%)

\section{Dietary Pattern(s):}

Alternative Healthy Eating Index for Pregnancy (AHEI-P):

- T1: $(n=285)$

- T2: $(n=286)$

- T3: $(n=282)$

\section{Results}

\section{Non-significant:}

GWG from first to second trimester $(\mathrm{kg}), \mathrm{P}=\mathrm{NS}$

Significant:

Non-significant:

GWG $(\mathrm{kg}), \mathrm{P}=\mathrm{NS}$

$\mathrm{T} 1: 13.4 \pm 6.4$
Confounding, Study Limitations, and Summary of Findings

\section{Limitations:}

- The study enrolled only those women who were at risk of GDM (i.e. women with a history of GDM and/or prepregnancy BMI $\geq 30$ ). The findings, therefore, may not be generalizable

- Potential selection bias due to exclusion after first trimester OGTT

- Women diagnosed with GDM may have altered their diet in an unbalanced way

- Potential differential exposure time between groups

- Reasons for/characteristics of women with missing outcome data NR

- Total GWG not measured

- No pre-registered data analysis plan.

\section{Summary:}

Adherence to the Healthy Food Intake Index during the first trimester was not associated with GWG from first to second trimester. 


\section{Study and Participant}

Characteristics $^{\mathrm{xi}}$

- Age: $29.1 \pm 5.4 y$

- Race/Ethnicity: White: $87.4 \%$

- SES:

- Education:

- High school or less: $18.0 \%$

- Some college: $39.3 \%$

- Associate or BA: $31.7 \%$

- Master or more: $10.9 \%$

- Poverty index ratio:

- <185\%: $37.5 \%$

- $\geq 350 \%: 23.7 \%$

- Anthropometry: Prepregnancy BMI:

$26.1 \pm 6.4$

- Smoking Status: Yes: 8.3\%

Rifas-Shiman, $2009^{6}$; U.S.

PCS, Project Viva

Baseline $\mathrm{N}=1777$ Analytic $\mathrm{N}=1666$

2,670 enrolled, then excluded 329 due to subsequent ineligibility. Additional women withdrew $(n=195)$, or were lost to follow-up $(n=18) .1777$ completed first FFQ, 1666 completed second FFQ

\section{Intervention/Exposure and Outcomes}

Based on a 130-point scale with $0-10$ points awarded for optimal intake of 13 types of

foods and nutrients

- Positively-scored components: vegetables, whole fruit, whole grains, nuts and legumes, long-chain ( $\mathrm{n}-3)$ fats, PUFAs, folate, calcium, and iron

- Negatively-scored components: sugarsweetened beverages, red and processed meat, trans fat, and sodium

at 28-36wk gestation

\section{Dietary assessment methods:}

Modified version of the Diet History

Questionnaire (DHQ) previously developed and validated by the National Cancer Institute (see Subar, 2001a; Subar, 2001b;

Thompson, 2002a; Thompson, 2002b)

\section{Outcome \& assessment methods:}

GWG at postpartum using neonatal questionnaire

\section{Dietary Pattern(s):}

Adherence to Alternate Healthy Eating Index for Pregnancy (AHEI-P)

- Based on 9 components: vegetables; fruit; ratio of white to red meat; fiber; trans fat; ratio of polyunsaturated to saturated fatty acids; and folate, calcium, and iron from foods (each 10 points, with a total of 90 points)
Results

T2: $14 \pm 6.1$

T3: $14.4 \pm 5.8$

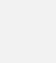

\section{Confounding, Study Limitations, and Summary of Findings}

- Baseline differences in BMI between groups and it is possible that the rate of weight gain might have differed based on their baseline

BMl, but not discussed or accounted for

- GWG is self-reported

\section{Summary:}

There was no association between adherence to the AHEI-P and GWG

\section{Significant:}

\section{Non-significant:}

$50 \%$ experienced excessive weight gain

GWG Adequacy for each 5 point increase in AHEI-P score (Ref: Adequate)

- First trimester $(n=1,777)$

\section{Key confounders accounted for:}

Age, Race/Ethnicity, SES, Pre-preg BMI

\section{Limitations:}

- Unclear whether those who did not complete FFQ in round 2 were different from those that completed first FFQ.

- Follow-up time varied across participants

- Reasons for exposure missingness NR

- Pre-registered data analysis plan NR 


\section{Study and Participant}

Characteristics $^{\mathrm{xi}}$

- Age: $32.4 \pm 4.9 y$

- Race/Ethnicity:

o White: $72 \%$

- Black/African American: 12\%

- Other, > 1 race: $16 \%$

- SES:

o Education:

- sHigh school diploma: 9\%

- College graduate: $69 \%$

- Household income <\$40,000/y: $13 \%$ $24.6 \pm 5.3$

- Parity

○ Nulliparous: $49 \%$

o $1: 36 \%$

$\circ \geq 2: 15 \%$
- Some college/tech school: $21 \%$

- Anthropometry: Prepregnancy BMI:

\section{Intervention/Exposure and Outcomes}

- Changes made to original AHEl: Alcohol, nuts excluded; folate, calcium and iron included

at first trimester ( 11wk) and second trimester ( 26-28wk)

\section{Dietary assessment methods:}

166-item validated semiquantitative $F F Q$, slightly modified for use in pregnancy

(Based on extensively validated Willett FFQ used in the Nurses' Health Study and calibrated against blood levels) (see Fawzi, 2004)

\section{Outcome \& assessment methods:}

Self-reported prepregnancy weight at baseline.

Last clinical prenatal weight recorded - selfreported prepregnancy weight

GWG adequacy based on IOM 1990 recommendations

\section{Sen, $2016^{9}$; U.S.}

PCS, Project Viva

Baseline $\mathrm{N}=1808$ Analytic $\mathrm{N}=1808$

$\mathrm{n}=\sim 1808$ for most participant characteristics

- Age: $32.2 \pm 5.0 y$

- Race/Ethnicity:

o Black: $13.9 \%$

o Hispanic: $6.8 \%$

\section{Dietary Pattern(s):}

Dietary inflammatory index (DII) score:

- Lower scores indicate lower dietary inflammation, characterized by higher intakes of vegetables, fruit, whole-grain lower intakes of red or processed meats and sugar-sweetened soda.

mean score from $\sim 9.9 \mathrm{wk}$ and $\sim 27.9 \mathrm{wk}$ gestation foods, fish/seafood, and whole eggs, and

\section{Results}

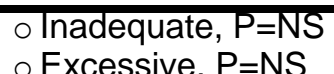

- Second trimester $(n=1,666)$

o Inadequate, $\mathrm{P}=\mathrm{NS}$

Excessive, $\mathrm{P}=\mathrm{NS}$

\section{Confounding, Study Limitations, and} Summary of Findings

\section{Summary:}

AHEI-P is not associated with pregnancy weight gain

\section{Significant:}

\section{Non-significant:}

GWG adequacy per unit of DII (Ref: Adequate):

- All women $(n=1808)$ - Inadequate, $\mathrm{P}=\mathrm{NS}$

- Excessive, $\mathrm{P}=\mathrm{NS}$

- Prepregnancy BMI 18.5$<25(n=1141)$ o Inadequate, $P=N S$ Excessive, $\mathrm{P}=\mathrm{NS}$

\section{Key confounders accounted for:}

Age, Race/ethnicity, SES, Pre-preg BMI, Smoking, Parity

\section{Limitations:}

- Data collected at the end of $2^{\text {nd }}$ trimester may be influenced by GDM knowledge

- Start of follow up and start of exposure may not coincide

- Self-reported diet and prepregnancy weight 


\begin{tabular}{|c|c|c|c|}
\hline $\begin{array}{l}\text { Study and Participant } \\
\text { Characteristics }\end{array}$ & Intervention/Exposure and Outcomes & Results & $\begin{array}{l}\text { Confounding, Study Limitations, and } \\
\text { Summary of Findings }\end{array}$ \\
\hline 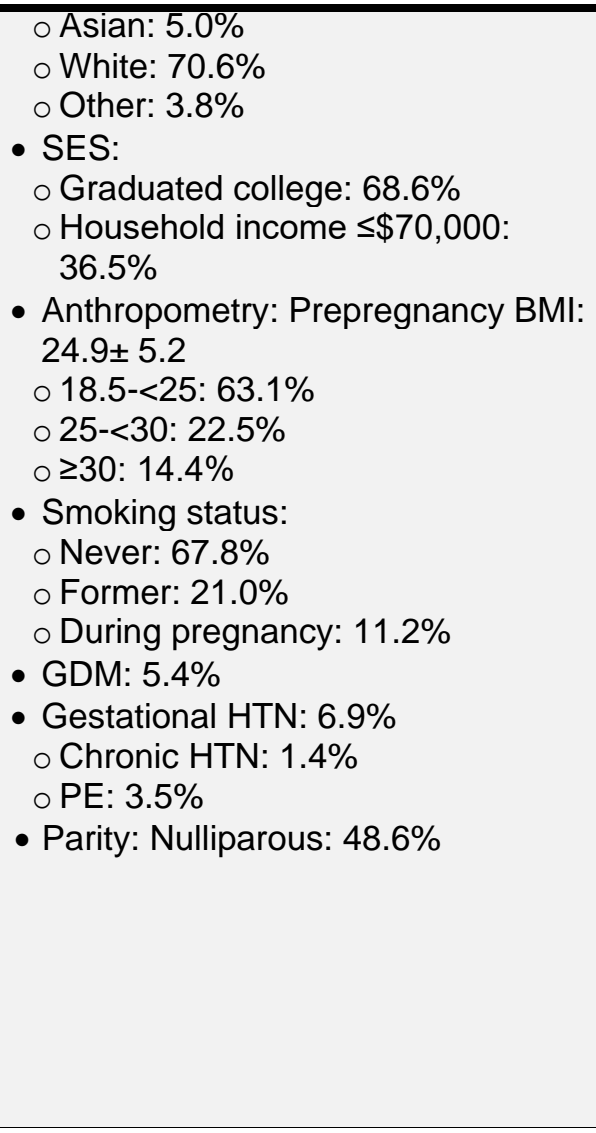 & $\begin{array}{l}\text { Dietary assessment methods: } \\
\text { Mothers completed self-administered FFQs at } \\
\text { median } 9.9 \text { wk and } 27.9 \text { wk gestation., } \\
\text { assessing diet intake during the first and } \\
\text { second trimesters. } \\
\text { Dietary data used to estimate a standard } \\
\text { global mean for the } 28 \text { food parameters } \\
\text { included in the DII, then converted to a } \\
\text { centered percentile score, multiplied by the } \\
\text { literature-derived respective food parameter } \\
\text { effect score to obtain a food parameter- } \\
\text { specific DII score, which were all summed to } \\
\text { create the overall DII score for each } \\
\text { participant. A higher DII score indicates a } \\
\text { more proinflammatory diet, whereas a more } \\
\text { negative score represents a more anti- } \\
\text { inflammatory diet. } \\
\text { Outcome \& assessment methods: } \\
\text { GWG (kg) calculated as the difference } \\
\text { between the last recorded clinical weight } \\
\text { before delivery and self-reported } \\
\text { prepregnancy weight reported at the first } \\
\text { study visit (9,9wk gestation). GWG adequacy } \\
\text { categorized by IOM (2009) } \\
\text { recommendations. }\end{array}$ & $\begin{array}{l}\text { - } \text { Prepregnancy BMI 25-<30 } \\
(\mathrm{n}=406) \\
\circ \text { Inadequate, } \mathrm{P}=\mathrm{NS} \\
\circ \text { Excessive, } \mathrm{P}=\mathrm{NS} \\
\text { - } \text { Prepregnancy } \mathrm{BMI} \geq 30 \\
(\mathrm{n}=261) \\
\text { - Inadequate, } \mathrm{P}=\mathrm{NS} \\
\circ \text { Excessive, } \mathrm{P}=\mathrm{NS}\end{array}$ & $\begin{array}{l}\text { - Important co-exposures were not balanced } \\
\text { across groups, and adjustment techniques } \\
\text { were used to correct for the issues. } \\
\text { - No information on reasons for missingness or } \\
\text { proportions across groups } \\
\text { - Any error in measuring the outcome is only } \\
\text { minimally related to exposure status } \\
\text { - No pre-registered data analysis plan } \\
\text { Summary: } \\
\text { Consumption of a low DII diet during the first } \\
\text { and second trimesters was not associated with } \\
\text { GWG. }\end{array}$ \\
\hline Tielemans, $2015^{8}$; Netherlands & Dietary Pattern(s): & Significant: & Key confounders accounted for: \\
\hline $\begin{array}{l}\text { Baseline } N=4097 \text { Analytic N=2748 } \\
\text { (Attrition: } 33 \% \text { ) }\end{array}$ & $\begin{array}{l}\text { - Adherence to standardized Dutch Healthy } \\
\text { Diet Index. Higher scores indicate better } \\
\text { adherence and higher intake of vegetables, } \\
\text { fruits, fiber, and fish, and lower intake of } \\
\text { saturated fat and sodium. } \\
\text { at } \sim 13.4 \mathrm{wk}\end{array}$ & $\begin{array}{l}\text { Non-significant: } \\
\text { Sensitivity Analysis (excludes } \\
\text { women with GDM or } \\
\text { hypertensive disorders of } \\
\text { pregnancy) }\end{array}$ & $\begin{array}{l}\text { Limitations: } \\
\text { - Self-reported diet, prepregnancy weight, and } \\
\text { maximum pregnancy weight }\end{array}$ \\
\hline
\end{tabular}




\section{Study and Participant}

Characteristics $^{\mathbf{x}}$

- Race/Ethnicity: $100 \%$ of Dutch ancestry

- SES:

o Education:

- Low and midlow: 18.2

- Midhigh: 51.5

- High: 30.4

o Household income <2200 Euro/mo 28.3

- Anthropometry: Prepregnancy BMI: 24.7

- Smoking status:

o Never in pregnancy: 74.4

o Until pregnancy known: 8.3

o During pregnancy: $\sim 17.4$

- Alcohol:

o Never in pregnancy: 36.6

o Until pregnancy known: 16.5

o During pregnancy: $\sim 46.9$

\section{Intervention/Exposure and Outcomes}

\section{Dietary assessment methods:}

The Dutch HDI was modified to align with pregnancy recommendations and available data, and consisted of 6 components: vegetable, fruit, dietary fiber, fish, saturated fatty acids, and sodium. The score of each component ranged between 0 and 10 points, resulting in a total score ranging from 0 to 60 points.

\section{Outcome \& assessment methods:}

Self-reported prepregnancy weight collected at enrollment. Staff measured weight at 3 study visits: $\sim 12.9 \mathrm{wk}$ (first visit), 20.4wk (second visit), and $\sim 30.2 w k$ (third visit). At 6wk postpartum, women self-reported maximum weight during pregnancy.

GWG in different phases of pregnancy was calculated mid-pregnancy GWG (weight at the second visit minus weight the first visit, divided by follow-up duration ( $\mathrm{g} /$ week), $\mathrm{n}=$ 2748), late-pregnancy GWG (weight at the third visit minus weight at the second visit, divided by follow-up duration ( $\mathrm{g} /$ week), $\mathrm{n}=$ 3158), and GWG until early-third trimester (weight at the third visit minus prepregnancy weight, divided by follow up duration (g/week), $n=2815$ ).

Women's total GWG (maximum pregnancy weight minus prepregnancy weight, $n=1917$ ) was used to classify their GWG into inadequate, adequate, or excessive GWG according to IOM (2009) recommendations.

\section{Results}

GWG until early-third

trimester (g/wk)

- Prepregnancy Normal

Weight Women $(n=1937)$

o Dutch HDI Pattern, $\mathrm{P}=0.06$

per SD increase in DP

score

- Q1: Ref

- Q2: $\beta=-13,95 \% \mathrm{Cl}:(-29$,

3)

- Q3: $\beta=-4,95 \% \mathrm{Cl}:(-20$,

12)

- Q4: $\beta=-16,95 \% \mathrm{Cl}:(-33$, 1)

- Prepregnancy Overweight Women $(n=532)$

Dutch HDI Pattern, $\mathrm{P}=\mathrm{NS}$ per $\mathrm{SD}$ increase in DP score
Confounding, Study Limitations, and Summary of Findings

\section{Summary:}

Adherence to Dutch HDI the was not associated with GWG. 


\section{Study and Participant}

Characteristics ${ }^{\mathrm{xi}}$

Intervention/Exposure and Outcomes

\section{Yong, 201920; Malaysia} PCS, SECOST

\section{Dietary Pattern(s)}

- Modified HEI for Malaysians comprised of 9 components, each with max score of 10
Results

\section{Non-significant}

Mid-Pregnancy Weight Gain

(g/wk)

- Prepregnancy Normal Weight Women $(n=2079)$

○ Dutch HDI Pattern, $P=0.76$ per SD

- Prepregnancy Overweight Women $(\mathrm{n}=669)$

o Dutch HDI Pattern, $P=0.88$ per SD

Late-Pregnancy Weight Gain (g/wk)

- Prepregnancy Normal Weight Women $(n=2384)$

○ Dutch HDI Pattern, $P=0.57$ per SD

- Prepregnancy Overweight Women $(n=774)$

o Dutch HDI Pattern, $\mathrm{P}=0.58$ per SD

GWG adequacy:

- Inadequate $\mathrm{GWG}(\mathrm{n}=459$, Ref: Adequate intake,

$\mathrm{n}=632$ )

Excessive GWG ( $\mathrm{n}=826$, Ref: Adequate intake, $\mathrm{n}=632$ )

\section{Confounding, Study Limitations, and} Summary of Findings

Age, Race/ethnicity, SES, Pre-preg BMI, Smoking, Parity

\section{Summary:}

Adherence to Dutch HDI the was not associated with GWG.

\section{Significant:}

Key confounders accounted for:

GWG adequacy by $\mathrm{HEl}$ in each trimester
Age, SES, PA, Pre-preg BMI, Parity 


\section{Study and Participant}

Characteristics $^{\mathbf{x i}}$

Baseline $\mathrm{N}=480$ Analytic $\mathrm{N}=480$

(Attrition: 0\%)

- Age: $30.16 \pm 4.51 \mathrm{y}$

- Race/Ethnicity:

o Malay: 89.0\%

o Non-Malay: $11.0 \%$

- SES:

○ Education level: $12.95 \pm 2.41 y$

- Secondary and lower: $46.0 \%$

- STPM/matric/diploma/certificate: $32.7 \%$

- Tertiary and above: $21.3 \%$

- Occupation status: Employed: $69.2 \%$

- Monthly household income: RM $3698.30 \pm 2034.20$

- Low: $63.5 \%$

- Middle: $33.5 \%$

- High: $2.9 \%$

o Household size: $3.78 \pm 1.63$

- $\leq 2: 24.2 \%$

- 3-4: $50.0 \%$

- $\geq 5: 25.8 \%$

- Physical Activity: MET hrs/week: o 2nd trimester 264.58 118.06 o 3rd trimester: 249.56 107.36

- Anthropometry:

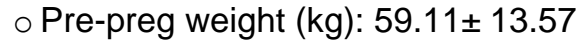

o Pre-preg BMI: 24.10 5.06

o Underweight: $10.2 \%$

o Normal: $53.1 \%$

o Overweight: $22.3 \%$

o Obese: $14.4 \%$

- GDM: History: $7.5 \%$

- Parity: $1.22 \pm 1.29$

\section{Intervention/Exposure and Outcomes}

and min score of 0 . Higher score indicates intake closer to recommended range.

o Adherence with the 7 major food groups recommended by Malaysian Dietary

Guidelines 2010 (MDG):

- Cereals and grains

- Vegetables

- Fruits

- Milk and milk products

- Poultry, meat and egg

- Fish and seafood

- Legumes

- Adherence with MDG recommendations for \%E from fat and sodium intake.

once during each trimester

\section{Dietary assessment methods:}

24-hr recall conducted each trimester. Dietary data analyzed using Nutritionist Pro Diet

Analysis software: Version 1.5.

Score for each $\mathrm{HEl}$ component calculated using formula: (Actual serving consumed based on respondent's diet recall/recommended serving size based on MDG) and multiplied by 10 . If an individual consumed less than the recommended amount of servings, score was calculated with the formula: $10 \times$ (the consumed amount of servings)/ (the lower limit of the recommended serving). If an individual consumed more than the recommended amount of servings, the score was calculated with the formula: 10-10 $\times$ [(the consumed servings) - (the upper limit of the recommended servings)]/(the upper limit of the recommended serving). Each score

Confounding, Study Limitations, and Summary of Findings

- All women

o 2nd trimester:

- Inadequate: $\mathrm{OR}=0.98$ $95 \% \mathrm{Cl}=(0.96,0.98)$, $\mathrm{P}=0.03$

o 3rd trimester:

- Excessive: OR=1.04, $95 \% \mathrm{Cl}=(1.01,1.06)$, $\mathrm{P}=0.01$

- Non-oveweight/obese $(\mathrm{n}=304)$ :

o 2nd trimester:

- Inadequate: OR=0.97 $95 \% \mathrm{Cl}=(0.95,0.99)$, $\mathrm{P}=0.01$

o 3rd trimester:

- Excessive: $\mathrm{OR}=1.04$, $95 \% \mathrm{Cl}=(1.01,1.07)$ $\mathrm{P}=0.03$

- Overweight/obese $(n=176)$ :

o 2nd trimester:

- Excessive: $\mathrm{OR}=1.04$, $95 \% \mathrm{Cl}=(1.01,1.07)$, $\mathrm{P}=0.02$

○ 3rd trimester:

- Excessive: OR=1.04, $95 \% \mathrm{Cl}=(1.01-1.08)$, $\mathrm{P}=0.02$

\section{Non-significant}

GWG adequacy by $\mathrm{HEI}$ in each trimester

- All women

o 1st trimester:

\section{Limitations:}

- Respondents were not representative of the general population of pregnant women in Malaysia.

- Self-reported dietary data; single 24-h dietary recall might not represent the usual intake

- Third trimester results may be crosssectional-unclear timing of third FFQ

- Start of follow up and start of exposure did not coincide and a potentially important amount of follow-up time is missing from analyses

- Co-exposures and reasons for missingness between groups NR

- High risk of selective reporting from among multiple analyses

- Information on missingness NR

- No pre-registered data analysis plan

\section{Summary:}

Women who were not overweight or obese prepregnancy with a higher total HEI score in the second trimester were at a lower risk for inadequate GWG, while women who were overweight or obese prepregnancy with a higher total $\mathrm{HEl}$ score in the second trimester were at a higher risk for excessive GWG. Women with a higher total $\mathrm{HEI}$ score in the third trimester were at higher risk for excessive GWG, regardless of prepregnancy BMI. 


\section{Study and Participant}

Characteristics $^{\mathrm{xi}}$

o $0: 36.7 \%$

○ $1-2: 47.7 \%$

$\circ \geq 3: 15.6 \%$

\section{Intervention/Exposure and Outcomes}

Results

calculation produced a negative score

because of excess servings, score converted to 0 .

\section{Outcome \& assessment methods:}

Prepregnancy weight self-reported. Weight measured at 10-13wks, 24-32wks, and 3438wks using standard instrument and procedures. GWG calculated as difference between measured weight at last visit and prepregnancy weight. rounded off to nearest whole number. When

- Inadequate, $P=N S$

- Excessive, $\mathrm{P}=\mathrm{NS}$

$\circ$ 2nd trimester:

- Excessive, $\mathrm{P}=\mathrm{NS}$

- 3rd trimester:

- Inadequate, $\mathrm{P}=\mathrm{NS}$

- Non-oveweight/obese $(n=304)$ :

o 1st trimester:

- Inadequate, $P=N S$

- Excessive, $\mathrm{P}=\mathrm{NS}$

$\circ$ 2nd trimester:

- Excessive, $\mathrm{P}=\mathrm{NS}$

o 3rd trimester:

- Inadequate, $\mathrm{P}=\mathrm{NS}$

- Overweight/obese $(n=176)$ :

o 1st trimester:

- Inadequate, $\mathrm{P}=\mathrm{NS}$

- Excessive, $\mathrm{P}=\mathrm{NS}$

$\circ$ 2nd trimester:

- Inadequate, $\mathrm{P}=\mathrm{NS}$

- 3rd trimester:

Inadequate, $\mathrm{P}=\mathrm{NS}$

\section{Zhu, 201916; U.S.}

\section{PCS, PETALS}

Baseline $\mathrm{N}=2269$ Analytic $\mathrm{N}=2269$

Of 2525 enrolled, loss to follow-up due to pregnancy loss ( $n=37$ ), moved $(n=7)$ or excluded due to missing data on child BW $(n=94), F F Q(n=87)$, $>6000 \mathrm{kcal} / \mathrm{d}$ reported $(n=23)$, or due to FFQ after GDM diagnosis ( $n=8)$

\section{Dietary Pattern(s):}

- Healthy Eating Index (HEI) index measures adherence to the USDA Dietary Guidelines for Americans. The HEI-2010 consists of 12 components (total fruit, whole fruit, total vegetables, greens and beans, whole grains, dairy, total protein foods, seafood and plant proteins, fatty acids, refined grains, sodium and empty calories from solid fats, alcohol and added sugars), with a maximum possible score of 100 . Alcohol

\section{Significant:}

\section{Non-significant:}

GWG (kg), $P=0.69$

- Q1: $13.2 \pm 6.7$

- Q3: $12.9 \pm 6.2$

- Q4: $13.1 \pm 6.0$
- Q2: $13.2 \pm 6.2$
Confounding, Study Limitations, and Summary of Findings

\section{Key confounders accounted for:} None

\section{Limitations:}

- Participant characteristics not balanced across groups

- Selection into the study related to exposure and outcome and could not be adjusted for in the analyses 


\section{Study and Participant}

Characteristics ${ }^{\mathrm{xi}}$

$\mathrm{n}=2269$ for all participants

characteristics

- Age:

- 18-24: 16.1\%

○ 25-29: 26.5\%

○ 30-34: $35.8 \%$

$\circ \geq 35: \sim 21.6 \%$

- Race/Ethnicity:

Non-Hispanic White: 22.3\%

Hispanic: 41.3\%

- African American: 9.4\%

- Asian/Pacific Islander: 23.6\%

o Other: $~ 3.5 \%$

- SES:

o Education:

- High School or less: $13.8 \%$

- Some college: $38.4 \%$

- College graduate or above: $\sim 47.9 \%$

o Household income (\$):

- <50,000: 32.7\%

- $\geq 150,000: \sim 15.9 \%$

- Physical Activity: 152.1 METs/wk

- Anthropometry: Pre-preg BMI:

$0<18.5:$ 2.7\%

- 18.5-24.9: $40.3 \%$

- 25.0-29.9: 28.9\%

o $\geq 30.0: \sim 28.1 \%$

- Smoking status: $0.5 \%$

- GDM: 10.8\%

- Gestational HTN: 10.8\%

- Parity: Nulliparity: $44.1 \%$

- Alcohol in pregnancy: 15.0\%

\section{Confounding, Study Limitations, and}

Summary of Findings

intake was excluded from the empty

calories component.

- Q1: Score of 37.5-64.4 ( $n=567)$

- Q2: Score of 64.5-71.7 $(n=567)$

- Q3: Score of 71.8-78.6 $(n=568)$

- Q4: Score of 78.7-94.2 ( $n=567)$

at $10-13 w k$ gestation

\section{Dietary assessment methods:}

Validated, 147 item Block FFQ administered at visit 1 (gestational wks 10-13). Nutrient intakes adjusted for total energy intake using the residual method. HEI-2010 score was calculated to assess diet quality.

\section{Outcome \& assessment methods:}

NR
- Start of follow up and exposure did not coincide and a potentially important amount of follow-up time was missing from analyses

- Dietary intake self-reported from one FFQ

- Important co-exposures not balanced across groups that were likely to impact the outcome and no adjustment techniques were used to correct for the issues

- Outcome assessment methods NR

- No pre-registered data analysis plan

\section{Summary:}

Maternal diet quality as measured by the HEI2010 in early pregnancy was not associated with GWG. 


\section{Study and Participant}

Characteristics $^{\mathrm{xi}}$

\section{Reduced Rank Regression}

\section{Starling, 2017'11; U.S.}

PCS

Baseline $\mathrm{N}=764$ Analytic $\mathrm{N}=764$

(Attrition: 0\%)

- Age: 28.6y

- Race/Ethnicity:

o Non-Hispanic White: 60\%

Hispanic: $21 \%$

Non-Hispanic Black: 12\%

o Other: $6 \%$

- SES:

- More than high school education: $75.3 \%$

- Physical Activity: METs/wk: 183

- Anthropometry: Prepregnancy BMI: $25.4 \pm 5.9$

- Smoking status: : 5.5\%

Parity: Multiparous: $62.8 \%$

Intervention/Exposure and Outcomes

Results

Confounding, Study Limitations, and

Summary of Findings

\section{Dietary Pattern(s):}

- Pattern 1: Higher consumption of healthy protein sources (poultry, nuts, and seeds) whole grains, cheese, citrus fruits, melons and berries, and other fruits, as well as added sugars and discretionary solid fat

- Pattern 2: Higher consumption of eggs, potatoes, other starchy vegetables, discretionary solid fat, citrus, melons and berries, and non-whole grains and yogurt, added sugars, soy products (tofu and meat analogs), dark-green vegetables, and whole grains

\section{at $17 \mathrm{wk}$ gestation}

\section{Dietary assessment methods:}

Dietary intake during pregnancy was assessed using the National Cancer Institute's Automated Self-Administered (ASA) 24h Recall. Analysis was restricted to participants who completed $\geq 2$ ASA24s

\section{Outcome \& assessment methods:}

Maternal weight during pregnancy was measured at each in-person study visit, and all weights measured at clinic visits were abstracted from the prenatal medical record by study personnel. Maternal weight before pregnancy was obtained either from medical records ( $90 \%)$ or from participant self-report at the first study visit $(\sim 10 \%)$. GWG was calculated by subtracting the prepregnancy weight from the last recorded maternal weight

\section{Significant:}

GWG (kg)

- Pattern 1, Ptrend<0.001

$\circ \mathrm{T} 1: 12.4 \pm 6.6$

○ T2: $14.7 \pm 5.6$

○ T3: $15.7 \pm 5.7$

- Pattern 2, Ptrend=0.03

o T1: $13.5 \pm 6.1$

○ T2: $14.7 \pm 5.6$

T3: $14.7 \pm 6.5$

- Pattern 1 correlated with greater GWG, $P<0.01$ $\circ r=0.22$

- Pattern 2 correlated with greater GWG, $P=0.02$ $\circ r=0.09$

\section{Non-significant}

\section{Key confounders accounted for:}

None

\section{Limitations:}

- GWG was not stratified by prepregnancy BM and the analysis did not stratify weight gain by inadequate, adequate and excess

- Co-exposures unbalanced between groups and likely to impact the outcome

- Missingness by exposure NR

- Unclear GDM prevalence

- No pre-registered data analysis plan

\section{Summary:}

Women with greater adherence to pattern 1 (tertile 3) had significantly greater GWG (ptrend $<0.001$ ). Similarly, women with greater adherence to pattern 2 (tertile 2 and tertile 3) had significantly greater GWG (ptrend=0.03) 
Study and Participant

Characteristics ${ }^{\mathrm{xi}}$
Intervention/Exposure and Outcomes

during pregnancy. Median number of days

between the last recorded weight and

delivery was $5 \mathrm{~d}$ (IQR 2-8 d).
Confounding, Study Limitations, and

Summary of Findings
Results

ays

ins

gs


Table 4. Description of evidence on the relationship between diets based on macronutrient distribution during pregnancy and gestational weight gain ${ }^{x i i}$

Study and Participant Characteristics ${ }^{\text {xiii }}$

\section{Poston, 201324; U.K.} RCT, UPBEAT

Baseline $\mathrm{N}=183$ Analytic $\mathrm{N}=140$ (Attrition: 23\%)

- Age: $30.5 y$

- Race/Ethnicity:

○ White: $~ 56 \%$

o Black: 38\%

- Asian: 2\%

o Other: $4 \%$

\section{- SES:}

o Most deprived: $~ 56 \%$

o Single: $\sim 46 \%$

- Living arrangements (\%):

o With partner: 74

- With parents: $~ 11$

- Housing (\%):

o Owned: 26

- Physical Activity ( $n=140)$ :

- Sedentary (min/d): 1169,

Active: 221, Light: 181,

MVPA: 41

- Anthropometry: BMI at $~ 17 \mathrm{wk}$ : $\sim 36.3$
Intervention/Exposure and Outcomes

Results

Significant:

Macronutrient Proportions:

- Control: $35.9 \%$ FAT, $48.2 \%$ CHO, $15.5 \%$ PRO $(n=69)$

- Intervention: $32.5 \%$ FAT, $50.0 \% \mathrm{CHO}$ $17.1 \%$ PRO $(n=71)$, also received PA intervention, to no effect

from $17 w k$ to $28 w k$ gestation

\section{Dietary assessment methods:}

Women randomized to intervention of dietary and physical activity advice delivered by health trainers vs standard care.

Diet assessed via repeated, triple pass, interviewer-led $24 \mathrm{hr}$ recall data obtained at baseline (randomization) and 28wk gestation. Diet evaluated twice, one week apart in both the intervention and control group.

\section{Outcomes \& assessment methods:}

Non-significant:

- Weight at $17 \mathrm{wk}(\mathrm{kg})$, Mean \pm SD

o Control: 96.8 16.2

o Intervention: $97.8 \pm 12.7$

- GWG at 28wk between groups, $\mathrm{P}=\mathrm{NS}$

o Data NR

GWG: Weight measured at baseline and 28wk
Confounding, Study Limitations, and Summary of findings

Key confounders accounted for: Age, Race/ethnicity, SES, PA, GDM, Parity

\section{Limitations:}

- Exploratory study, no power calculation for GWG

- Deviations from intended intervention unbalanced between groups and likely to have affected the outcome

- Unclear how weight was assessed and GWG was calculated

- No pre-registered data analysis plan

\section{Summary:}

Consuming a diet with $>35 \%$ FAT versus a diet with $<35 \%$ FAT during send trimester in obese women did not affect GWG.

xii BMI: body mass index, CHO: carbohydrate, d: day, GDM: gestational diabetes mellitus, g: gram, GWG: gestational weight gain, hr: hour, kg: kilogram(s), kJ: kilojoule(s), min: minute(s), MVPA: moderate to vigorous physical activity, PA: physical activity, PRO: protein, SES: socioeconomic status, UPBEAT: U.K. Pregnancies Better Eating and Activity Trial, wk: week(s), y: year(s)

xiii \pm indicates values of Mean \pm SD unless otherwise noted 
- GDM: at 28wk: $30 \%$

- Parity: 0: $44 \%, 1: 36 \%, \geq 2$ : $21 \%$

\section{Tajima, 2017 ${ }^{4}$; Japan PCS}

Baseline $\mathrm{N}=325$ Analytic $\mathrm{N}=325$

- Age: $~ 33.5 y$

- Race/Ethnicity: $100 \%$

Japanese

- SES: NR

- Anthropometry: BMI at first prenatal visit: $\sim 19.7$ $0<18.5: 28.9 \%$ $\circ \geq 23: 5.5 \%$

- GDM: $4 \%(n=540)$, Prepregnancy diabetes: 0\%

- Parity: Primiparous: $~ 67.1 \%$

\section{Macronutrient Proportions:}

- Bottom: $48.9 \%$ CHO, 35.1\% FAT, $16.0 \%$ PRO $(n=108)$

- Middle: $54.9 \%$ CHO, $30.3 \%$ FAT, $14.8 \%$ PRO $(n=109)$

- Top: $61.5 \%$ CHO, $24.6 \%$ FAT, $13.9 \%$ PRO $(\mathrm{n}=108)$

at $<16$ wk gestation

\section{Dietary assessment methods:}

Data grouped by tertile of $\mathrm{CHO}$ intake (bottom, middle, and top).

3 -d weighed dietary record on 2 weekdays and 1 weekend day, consecutive or nonconsecutive. Calories from each macronutrient calculated by multiplying $\mathrm{g}$ of macronutrients by calories $/ \mathrm{g}(16.7 \mathrm{~kJ} / \mathrm{g}$ for protein; $37.7 \mathrm{~kJ} / \mathrm{g}$ for fat). Percentage of carbohydrate intake calculated by subtracting percentages of protein and fat intake from $100 \%$.

\section{Outcomes \& assessment methods:}

GWG: Weight measured by a registered nurse at the first prenatal visit and at 28wk. Rate of GWG/wk calculated from $1^{\text {st }}$ prenatal visit to 28 wks of gestation.

\section{Significant:}

\section{Non-significant:}

- GWG per week (kg), P=0.22

o Bottom: $0.3 \pm 0.1$

o Middle: $0.3 \pm 0.1$

o Top: $0.3 \pm 0.1$
Key confounders accounted for:

Race/ethnicity

\section{Limitations:}

- Self-reported dietary assessments during first trimester, when women may be prone to nausea

- Start of follow up and exposure did not coincide and a potentially important amount of follow-up time is missing from analyses

- Methods used to define exposure status are not clearly described and likely to result in some degree of random misclassification

- GWG not stratified by prepregnancy $\mathrm{BMI}$ and unclear if it varied among underweight vs. healthy vs. overweight participants

- GWG available for a small window ( 728 wks) and weight gain during first trimester may have been minimal

- Generalizability to U.S. population may be questionable given that $\sim 1 / 3$ of the participants were underwight

- No information on whether the proportion of participants and reasons for missing data are similar across exposure groups 
Study and Participant

Characteristics ${ }^{\mathrm{xiii}}$

Intervention/Exposure and Outcomes

Results

Confounding, Study Limitations, and Summary of findings

Summary:

GWG per week is not significantly

different between tertiles of \%CHO among Japanese pregnant women. 
Table 5. Risk of bias for randomized controlled trials examining dietary patterns during pregnancy and gestational weight gain ${ }^{\text {xiv xv }}$

\begin{tabular}{|l|c|c|c|c|c|}
\hline & Randomization & $\begin{array}{c}\text { Deviations from } \\
\text { intended } \\
\text { interventions }\end{array}$ & $\begin{array}{c}\text { Missing } \\
\text { outcome data }\end{array}$ & $\begin{array}{c}\text { Outcome } \\
\text { measurement }\end{array}$ & $\begin{array}{c}\text { Selection of the } \\
\text { reported result }\end{array}$ \\
\hline Al Wattar, 201922 & Low & Low & Low & Some concerns & Low \\
\hline Assaf-Balut, 201726 & Low & High & Low & Low & Low \\
\hline Van Horn, 201825 & Low & High & Low & Low & Low \\
\hline Poston, 201324 & Low & High & Low & Some concerns & Some concerns \\
\hline Assaf-Balut, 201923 & Low & High & Low & Low & High \\
\hline
\end{tabular}

Table 6. Risk of bias for observational studies examining dietary patterns during pregnancy and gestational weight gain ${ }^{x v i}$

\begin{tabular}{|c|c|c|c|c|c|c|c|}
\hline & Confounding & $\begin{array}{l}\text { Selection of } \\
\text { participants }\end{array}$ & $\begin{array}{l}\text { Classification } \\
\text { of exposures }\end{array}$ & $\begin{array}{l}\text { Deviations } \\
\text { from intended } \\
\text { exposures }\end{array}$ & Missing data & $\begin{array}{c}\text { Outcome } \\
\text { measurement }\end{array}$ & $\begin{array}{c}\text { Selection of } \\
\text { the reported } \\
\text { result }\end{array}$ \\
\hline Maugeri, $2019^{19}$ & Serious & Serious & Low & Low & Low & Moderate & Serious \\
\hline Okubo, $2012^{3}$ & Serious & Serious & Serious & Serious & Moderate & Serious & Serious \\
\hline Tielemans, $2015^{8}$ & Serious & Moderate & Moderate & Low & Low & Serious & Serious \\
\hline Wei, 2019 & Serious & Serious & Serious & No information & Serious & Moderate & Serious \\
\hline Wesolowska, 2019'17 & Critical & Serious & Moderate & Serious & Moderate & No information & Serious \\
\hline Ancira-Moreno, $2019^{18}$ & Serious & Serious & Serious & Low & Moderate & Moderate & Serious \\
\hline Emond, $2018^{13}$ & Serious & Serious & Moderate & Low & No information & Low & Serious \\
\hline
\end{tabular}

${ }^{\text {xiv }}$ A detailed description of the methodology used for assessing risk of bias is available on the NESR website: https://nesr.usda.gov/2020-dietary-guidelinesadvisory-committee-systematic-reviews and in Part C of the following reference: Dietary Guidelines Advisory Committee. 2020. Scientific Report of the 2020 Dietary Guidelines Advisory Committee: Advisory Report to the Secretary of Agriculture and the Secretary of Health and Human Services. U.S. Department of Agriculture, Agricultural Research Service, Washington, DC.

${ }_{x v}$ Possible ratings of low, some concerns, or high determined using the "Cochrane Risk-of-bias 2.0" (RoB 2.0) (August 2016 version)" (Higgins JPT, Sterne JAC, Savović J, Page MJ, Hróbjartsson A, Boutron I, Reeves B, Eldridge S. A revised tool for assessing risk of bias in randomized trials In: Chandler J, McKenzie J, Boutron I, Welch V (editors). Cochrane Methods. Cochrane Database of Systematic Reviews 2016, Issue 10 (Suppl 1). dx.doi.org/10.1002/14651858.CD201601.)

xvi Possible ratings of low, moderate, serious, critical, or no information determined using the "Risk of Bias for Nutrition Observational Studies" tool (RoBNObs) (Dietary Guidelines Advisory Committee. 2020. Scientific Report of the 2020 Dietary Guidelines Advisory Committee: Advisory Report to the Secretary of Agriculture and the Secretary of Health and Human Services. U.S. Department of Agriculture, Agricultural Research Service, Washington, DC.) 


\begin{tabular}{|c|c|c|c|c|c|c|c|}
\hline & Confounding & $\begin{array}{l}\text { Selection of } \\
\text { participants }\end{array}$ & $\begin{array}{l}\text { Classification } \\
\text { of exposures }\end{array}$ & $\begin{array}{l}\text { Deviations } \\
\text { from intended } \\
\text { exposures }\end{array}$ & Missing data & $\begin{array}{c}\text { Outcome } \\
\text { measurement }\end{array}$ & $\begin{array}{c}\text { Selection of } \\
\text { the reported } \\
\text { result }\end{array}$ \\
\hline $\begin{array}{l}\text { Fernández-Barrés, } \\
2019^{1}\end{array}$ & Critical & Serious & Serious & Serious & Moderate & Moderate & Serious \\
\hline Fulay, $2018^{12}$ & Serious & Moderate & Moderate & Low & Moderate & Moderate & Serious \\
\hline Gesteiro, 2012² & Serious & Serious & Serious & Low & Moderate & Low & Serious \\
\hline Hillesund, $2018^{21}$ & Serious & Moderate & Serious & Moderate & Serious & Moderate & Serious \\
\hline Hillesund, 20145 & Serious & Serious & Moderate & Moderate & Moderate & Serious & Serious \\
\hline Hrolfsdottir, 201914 & Serious & Moderate & Moderate & Low & Moderate & Moderate & Serious \\
\hline Meinila, $2017^{27}$ & Critical & Serious & Moderate & Moderate & No information & Moderate & Serious \\
\hline Poon, 20137 & Critical & Serious & Serious & Serious & Moderate & Serious & Serious \\
\hline Rifas-Shiman, $2009^{6}$ & Serious & Serious & Moderate & Moderate & Moderate & Moderate & Serious \\
\hline Sen, $2016^{9}$ & Serious & Moderate & Moderate & Moderate & Moderate & Moderate & Serious \\
\hline Yong, 201920 & Serious & Serious & Moderate & No information & No information & Moderate & Serious \\
\hline Zhu, 201916 & Critical & Moderate & Moderate & Low & Low & No information & Serious \\
\hline Starling, $2017^{11}$ & Critical & Moderate & Moderate & Moderate & Moderate & Moderate & Serious \\
\hline Tajima, $2017^{4}$ & Critical & Moderate & Serious & Moderate & No information & Low & Serious \\
\hline
\end{tabular}




\section{METHODOLOGY}

The NESR team used its rigorous, protocol-driven methodology to support the 2020 Dietary Guidelines Advisory Committee in conducting this systematic review.

NESR's systematic review methodology involves:

- Developing a protocol,

- Searching for and selecting studies,

- Extracting data from and assessing the risk of bias of each included study,

- Synthesizing the evidence,

- Developing conclusion statements,

- Grading the evidence underlying the conclusion statements, and

- Recommending future research.

A detailed description of the methodology used in conducting this systematic review is available on the NESR website: https://nesr.usda.gov/2020-dietary-guidelines-advisory-committee-systematicreviews, and can be found in the 2020 Dietary Guidelines Advisory Committee Report, Part C: Methodology.xvii Additional information about this systematic review, including a description of and rationale for any modifications made to the protocol can be found in the 2020 Dietary Guidelines Advisory Committee Report, Chapter 2. Food, Beverage, and Nutrient Consumption During Pregnancy.

Below are details of the final protocol for the systematic review described herein, including the:

- Analytic framework

- Literature search and screening plan

- Literature search and screening results

\section{ANALYTIC FRAMEWORK}

The analytic framework (Figure 1) illustrates the overall scope of the systematic review, including the population, the interventions and/or exposures, comparators, and outcomes of interest. It also includes definitions of key terms and identifies key confounders considered in the systematic review. The inclusion and exclusion criteria that follow provide additional information about how parts of the analytic framework were defined and operationalized for the review.

xvii Dietary Guidelines Advisory Committee. 2020. Scientific Report of the 2020 Dietary Guidelines Advisory Committee: Advisory Report to the Secretary of Agriculture and the Secretary of Health and Human Services. U.S. Department of Agriculture, Agricultural Research Service, Washington, DC. 


\section{Figure 1: Analytic framework}

\section{Systematic review question: What is the relationship between dietary patterns consumed during}

\section{pregnancy and gestational weight gain (GWG)?}

\begin{tabular}{|c|c|c|}
\hline Intervention/exposure & vs & Comparator \\
\hline $\begin{array}{l}\text { Consumption of and/or } \\
\text { adherence to a dietary pattern. }\end{array}$ & & $\begin{array}{l}\text { - Consumption of and/or } \\
\text { adherence to a different } \\
\text { dietary pattern } \\
\text { - Different levels of } \\
\text { consumption of and/or } \\
\text { adherence to a dietary } \\
\text { pattern }\end{array}$ \\
\hline
\end{tabular}

Population: Women during pregnancy; healthy and/or at risk for chronic disease

$\longrightarrow$
Outcomes
- Gestational weight gain: Change in maternal body weight from baseline (before or during pregnancy) to a later time point during pregnancy and/or right before delivery
- Weight gain in relationship to weight gain recommendations, based on pre- pregnancy BMl

Population: Women during pregnancy; healthy and/or at risk for chronic disease

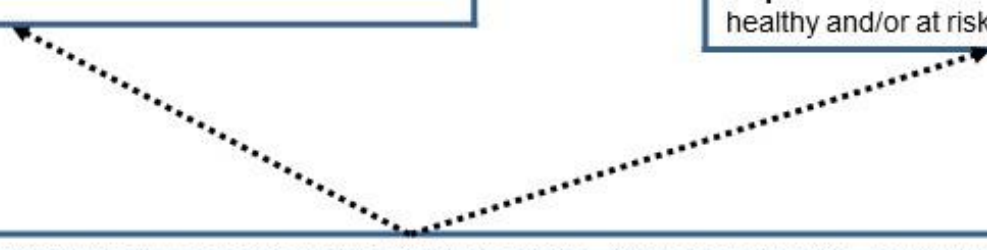

Key Confounders: Age, Race/ethnicity, Socioeconomic status, Physical activity, Anthropometry (Pre-pregnancy BMI), Smoking, History/diagnosis of gestational diabetes and gestational hypertension, Parity

\section{Key definitions}

Dietary Pattern - The quantities, proportions, variety, or combination of different foods, drinks, and nutrients (when available) in diets, and the frequency with which they are habitually consumed

\section{Legend}

$\longrightarrow$ The relationship of interest in the systematic review

....... Factors that may impact the relationship of interest in the systematic review 


\section{LITERATURE SEARCH AND SCREENING PLAN}

\section{Inclusion and exclusion criteria}

This table provides the inclusion and exclusion criteria for the systematic review. The inclusion and exclusion criteria are a set of characteristics used to determine which articles identified in the literature search were included in or excluded from the systematic review.

\section{Table 7. Inclusion and exclusion criteria}

\begin{tabular}{|c|c|c|}
\hline Category & Inclusion Criteria & Exclusion Criteria \\
\hline $\begin{array}{l}\text { Study } \\
\text { design }\end{array}$ & $\begin{array}{l}\text { - Randomized controlled trials } \\
\text { - Non-randomized controlled trials } \\
\text { including quasi-experimental and } \\
\text { controlled before-and-after studies } \\
\text { - Prospective cohort studies } \\
\text { - } \quad \text { Retrospective cohort studies } \\
\text { - Nested case-control studies }\end{array}$ & $\begin{array}{l}\text { - Uncontrolled trials } \\
\text { - } \quad \text { Case-control studies } \\
\text { - } \text { Cross-sectional studies } \\
\text { - Uncontrolled before-and-after } \\
\text { studies } \\
\text { - Narrative reviews } \\
\text { - Systematic reviews } \\
\text { - } \quad \text { Meta-analyses }\end{array}$ \\
\hline $\begin{array}{l}\text { Intervention/ } \\
\text { exposure }\end{array}$ & $\begin{array}{l}\text { - Studies that examine consumption of } \\
\text { and/or adherence to a } \\
\text { 1. Dietary pattern [i.e., the } \\
\text { quantities, proportions, variety, or } \\
\text { combination of different foods, } \\
\text { drinks, and nutrients (when } \\
\text { available) in diets, and the } \\
\text { frequency with which they are } \\
\text { habitually consumed] including, at } \\
\text { a minimum, a description of the } \\
\text { foods and beverages in the } \\
\text { pattern }\end{array}$ & $\begin{array}{l}\text { Studies that } \\
\text { 1a. Do not provide a description } \\
\text { of the dietary pattern, which at } \\
\text { minimum, must include the foods } \\
\text { and beverages in the pattern } \\
\text { (i.e., studies that examine a } \\
\text { labeled dietary pattern, but do } \\
\text { not describe the foods and } \\
\text { beverages consumed) }\end{array}$ \\
\hline & \multicolumn{2}{|l|}{ and/or } \\
\hline & $\begin{array}{l}\text { 2. Diet based on macronutrient } \\
\text { distribution outside of the AMDR } \\
\text { and } \\
\text { - Include the macronutrient } \\
\text { distribution of carbohydrate, } \\
\text { fat, and protein of the diet, and } \\
\text { - Include at least one } \\
\text { macronutrient outside of the }\end{array}$ & $\begin{array}{l}\text { 2a. Examine consumption of } \\
\text { and/or adherence to a diet based } \\
\text { on macronutrient proportion in } \\
\text { which all macronutrients are } \\
\text { within the AMDR } \\
\text { 2b. Do not describe the entire } \\
\text { macronutrient distribution of the } \\
\text { diet (i.e., studies that only } \\
\text { examine a single macronutrient } \\
\text { in relation to outcomes) }\end{array}$ \\
\hline
\end{tabular}




\begin{tabular}{|c|c|c|}
\hline Category & Inclusion Criteria & Exclusion Criteria \\
\hline \multicolumn{3}{|c|}{$\begin{array}{l}\text { acceptable macronutrient } \\
\left.\text { distribution range (AMDR }{ }^{\text {xviii }}\right)\end{array}$} \\
\hline Comparator & $\begin{array}{l}\text { Dietary patterns described by foods and } \\
\text { beverages consumed: } \\
\text { - Consumption of and/or adherence to } \\
\text { a different dietary pattern } \\
\text { - Different levels of consumption of } \\
\text { and/or adherence to a dietary pattern } \\
\text { Diets described by macronutrient } \\
\text { distribution: } \\
\text { - Different macronutrient distribution of } \\
\text { carbohydrate, fat, and protein }\end{array}$ & $\begin{array}{l}\text { - } \quad \text { No comparator } \\
\text { - Macronutrient proportion(s) of } \\
\text { interest also outside the AMDR }\end{array}$ \\
\hline Outcomes & $\begin{array}{l}\text { - Change in maternal body weight } \\
\text { from baseline (before or during } \\
\text { pregnancy) to a later time point } \\
\text { during pregnancy and/or right before } \\
\text { delivery } \\
\text { - Weight gain in relationship to weight } \\
\text { gain recommendations, based on } \\
\text { pre-pregnancy BMI }\end{array}$ & $\begin{array}{l}\text { - Changes in weight from any point } \\
\text { during pre-pregnancy or } \\
\text { pregnancy to postpartum period }\end{array}$ \\
\hline $\begin{array}{l}\text { Date of } \\
\text { publication }\end{array}$ & - January 2000-November 2019 & $\begin{array}{l}\text { - Articles published before January } \\
2000 \text { or after November } 2019\end{array}$ \\
\hline $\begin{array}{l}\text { Publication } \\
\text { status }\end{array}$ & $\begin{array}{l}\text { - Articles that have been peer- } \\
\text { reviewed }\end{array}$ & $\begin{array}{l}\text { Articles that have not been peer- } \\
\text { reviewed and are not published } \\
\text { in peer-reviewed journals, } \\
\text { including unpublished data, } \\
\text { manuscripts, reports, abstracts, } \\
\text { and conference proceedings }\end{array}$ \\
\hline $\begin{array}{l}\text { Language of } \\
\text { publication }\end{array}$ & - Articles published in English & $\begin{array}{l}\text { - Articles published in languages } \\
\text { other than English }\end{array}$ \\
\hline
\end{tabular}

xviii Macronutrient percent of energy outside of the AMDR are as follows:

- Carbohydrate for all age groups: $<45$ or $>65$ percent of energy;

- Protein (age 19 years and older): $<10$ or $>35$ percent of energy;

- Fat (age 19 years and older): $<20$ or $>35$ percent of energy.

Institute of Medicine. Dietary Reference Intakes for Energy, Carbohydrate, Fiber, Fat, Fatty Acids, Cholesterol, Protein, and Amino Acids. Washington, DC: The National Academies Press; 2002. 


\section{$\begin{array}{lll}\text { Category Inclusion Criteria } & \text { Exclusion Criteria }\end{array}$}

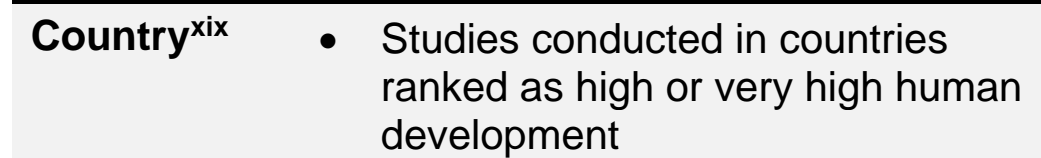

\begin{tabular}{ll}
\hline Study \\
participants
\end{tabular}

- Studies conducted in countries ranked as medium or lower human development

- Animal and in vitro models

- Pregnancies conceived ONLY using Assisted Reproductive Technologies

- Studies that exclusively enroll multiple gestation pregnancies

- Studies that enroll both singleton and multiple pregnancies and do not account for singleton and multiple gestation in the design or analyses and only present aggregate findings

Health
status of
study
participants

- Studies that enroll mothers who are healthy and/or at risk for chronic disease

- Studies that enroll some mothers diagnosed with a disease

- Studies that enroll some mothers who were severely undernourished prior to pregnancy

- Studies that enroll some or all mothers classified as underweight, or obese prior to pregnancy
- Studies that ONLY enroll mothers who gave birth to preterm ( $<37$ weeks)

- Studies that ONLY enroll mothers diagnosed with a disease, including severe undernutrition or hospitalized with an illness or injury
Studies that assess exposure prior to outcome
- Studies that assess outcome prior to exposure

xix The Human Development classification was based on the Human Development Index (HDI) ranking from the year the study intervention occurred or data were collected (UN Development Program. HDI 1990-2017 HDRO calculations based on data from UNDESA (2017a), UNESCO Institute for Statistics (2018), United Nations Statistics Division (2018b), World Bank (2018b), Barro and Lee (2016) and IMF (2018). Available from:

http://hdr.undp.org/en/data). If the study did not report the year in which the intervention occurred or data were collected, the HDI classification for the year of publication was applied. HDI values are available from 1980, and then from 1990 to present. If a study was conducted prior to 1990, the HDI classification from 1990 was applied. When a country was not included in the HDI ranking, the current country classification from the World Bank was used instead (The World Bank. World Bank country and lending groups. Available from:

https://datahelpdesk.worldbank.org/knowledgebase/articles/906519-world- country-and-lending-groups). 


\section{Electronic databases and search terms}

\section{PubMed}

- Provider: U.S. National Library of Medicine

- Date(s) searched: June 26, 2019, Update: November 7, 2019

- Date range searched: January 1, 2000 - June 26, 2019; Update: January 1, 2000 November 7, 2019

- Search terms:

\#1 - dietary pattern* OR diet pattern* OR eating pattern* OR food pattern* OR diet quality[tiab] OR eating habit*[tiab] OR dietary habit* OR diet habit* OR food habit* OR "Feeding Behavior"[Mesh] OR feeding behavior*[tiab] OR beverage consumption[tiab] OR beverage habit*[tiab] OR beverage intake*[tiab] OR dietary profile* OR food profile[tiab] OR diet profile* OR eating profile* OR dietary guideline* OR dietary recommendation* OR dietary intake[tiab] OR food intake[tiab] OR food consumption[tiab] OR dietary consumption[tiab] OR eating frequenc* OR food frequenc*[tiab] OR eating style*[tiab] OR dietary change ${ }^{\star}\left[\right.$ tiab] OR dietary choice ${ }^{\star}[$ tiab] OR food choice*[tiab] OR "Diet, Mediterranean"[Mesh] OR Mediterranean Diet*[tiab] OR "Dietary Approaches To Stop Hypertension"[Mesh] OR Dietary Approaches To Stop Hypertension Diet* OR DASH diet $^{\star}$ OR "Diet, Gluten-Free"[Mesh] OR Gluten Free $\operatorname{diet}^{\star}$ OR prudent diet* OR "Diet, Paleolithic"[Mesh] OR Paleolithic Diet* OR "Diet, Vegetarian"[Mesh] OR vegetarian diet*[tiab] OR vegan diet* OR "Healthy Diet"[Mesh] OR plant based diet* OR "Diet, Western"[Mesh] OR western $\operatorname{diet}^{*}$ OR "Diet, Carbohydrate-Restricted"[Mesh] OR low-carbohydrate diet* OR high carbohydrate diet $^{\star}$ OR Ketogenic Diet* OR Nordic Diet* OR "Diet, Fat-Restricted"[Mesh] OR "Diet, High-Fat"[Mesh] OR "Diet, High-Protein"[Mesh] OR high protein diet*[tiab] OR high-fat $\operatorname{diet}^{*}$ [tiab] OR low fat diet*[tiab] OR "Diet, Protein-Restricted"[Mesh] OR low protein $\operatorname{diet}^{\star}$ OR "Diet, Sodium-Restricted"[Mesh] OR low-sodium diet* OR low salt diet* OR (("Dietary Proteins"[Mesh] OR dietary protein*[tiab] OR "Dietary Carbohydrates"[Mesh] OR dietary carbohydrate*[tiab] OR "Dietary Fats"[Mesh] OR dietary fat"[tiab] OR hypocaloric OR hypocaloric) AND (diet[tiab] OR diets[tiab] OR consumption[tiab] OR intake[tiab] OR supplement*[tiab])) OR ("Guideline Adherence"[Mesh] AND (diet[tiab] OR dietary[tiab] OR food[tiab] OR beverage[tiab])) OR (diet score* OR diet quality score* OR diet quality index* OR dietary habits score* OR kidmed OR diet index* OR dietary index* OR Food-based Index* OR diet quality index* OR food index* OR food score* OR Mediterranean diet score* OR MedDietScore OR healthy eating index[tiab] OR food frequency questionnaire* OR food frequency survey* OR "Nutrition Surveys"[Mesh] OR nutrition survey*[tiab] OR diet survey*[tiab] OR food survey* OR dietary questionnaire[tiab]) OR ((pattern[tiab] OR patterns[tiab] OR consumption[tiab] OR habit*[tiab]) AND ("Diet"[Mesh:NoExp] OR diet[tiab] OR diets[tiab] OR dietary[tiab] OR "Food"[Mesh] OR food[tiab] OR foods[tiab] OR "Beverages"[Mesh] OR beverage[tiab] OR beverages[tiab]))

\#2 - "Pregnancy"[Mesh] OR "Pregnancy Complications"[Mesh] OR "Prenatal Exposure Delayed Effects"[Mesh] OR "Maternal Exposure"[Mesh] OR "pregnant women"[Mesh] OR pregnan*[tiab] OR pre-pregnancy[tiab] OR prenatal[tiab] OR pre-natal[tiab] OR maternal[tiab] OR mother[tiab] OR mothers[tiab] OR "Mothers"[Mesh] OR postpartum[tiab] OR perinatal[tiab] OR peri-natal[tiab] OR pre-conception[tiab] OR preconception[tiab] OR peri-conception[tiab] OR periconception[tiab] OR "Peripartum Period"[Mesh] OR peripartum[tiab] OR peri-partum[tiab] OR gestation*[tiab] OR natal[tiab] OR antenatal[tiab] OR ante-natal[tiab] OR puerperium[tiab] OR 
"Maternal Nutritional Physiological Phenomena"[Mesh] OR "Postpartum Period"[Mesh] OR postpartum[tiab] OR post-partum[tiab] OR perinatal OR peri-natal OR puerperium[tiab] OR postpartal OR post-partal OR postnatal OR post delivery[tiab] OR after birth[tiab] OR "Lactation"[Mesh] OR lactation[tiab] OR lactating[tiab] OR "Breast Feeding"[Mesh] OR breastfeeding[tiab] OR breast-feeding[tiab] OR breast feed ${ }^{*}$ OR breast-feed*[tiab] OR breastfed[tiab] OR breast-fed[tiab] OR breastfeed* OR "Milk, Human"[Mesh] OR human milk[tiab] OR nursing women[tiab]

\#3 - "Gestational Weight Gain"[Mesh] OR gestational weight gain[tiab] OR "Weight Gain"[Mesh:NoExp] OR weight gain[tiab] OR "Obesity"[Mesh] OR obesity[tiab] OR obese[tiab] OR overweight[tiab] OR "body size"[tiab] OR "Body Size"[Mesh] OR overnutrition[tiab] OR "Overnutrition"[Mesh:NoExp] OR adipos*[tiab] OR anthropometry[tiab] OR anthropometric*[tiab] OR "Adiposity"[Mesh] OR adipose[tiab] OR body weight[tiab] OR "Body Weight"[Mesh] OR "Body Composition"[Mesh] OR body fat[tiab] OR weight[ti] OR "Body Mass Index"[Mesh] OR body mass index[tiab] OR BMI[tiab] OR weight status[tiab] OR "Adipose Tissue"[Mesh] OR healthy weight[tiab] OR body fat mass[tiab] OR weight change[tiab] OR weight changes[tiab] OR "Weight Loss"[Mesh] OR weight loss*[tiab] OR weight reduc*[tiab] OR body weight[tiab] OR "Weight Reduction Programs"[Mesh] OR "Body-Weight Trajectory"[Mesh] OR weight maint* OR "Diet, Reducing"[Mesh] OR diet reduc*[tiab] OR weight cycling[tiab] OR weight decreas*[tiab] OR weight watch*[tiab] OR weight control*[tiab] OR weight retention[tiab] OR (weight[tiab] AND (reduction OR reduced OR reducing OR loss OR losses OR maintenanc* OR maintain*[tiab] OR decreas*$^{*}[$ tiab] OR watch OR control*[tiab] OR change*[tiab] OR gain[tiab]))

\section{\#4 - \#1 AND \#2 AND \#3}

\#5 - \#4 NOT ("Animals"[Mesh] NOT ("Animals"[Mesh] AND "Humans"[Mesh])) NOT (editorial[ptyp] OR comment[ptyp] OR news[ptyp] OR letter[ptyp] OR review[ptyp] OR systematic review[ptyp] OR systematic review[ti] OR meta-analysis[ptyp] OR meta-analysis[ti] OR metaanalyses[ti] OR retracted publication[ptyp] OR retraction of publication[ptyp] OR retraction of publication[tiab] OR retraction notice[ti]) Filters: Publication date from 2000/01/01 to 2019/06/26; English, Update: Filters: Publication date from 2000/01/01 to 2019/11/07; English

\section{Cochrane Central Register of Controlled Trials (CENTRAL)}

- Provider: John Wiley \& Sons

- Date(s) searched: June 26, 2019; Update: November 7, 2019

- Date range searched: January 1, 2000 - June 26, 2019; Update: January 1, 2000 November 7, 2019

- Search terms:

\#1 - "dietary pattern*" OR "diet pattern*" OR "eating pattern*" OR "food pattern*" OR "diet quality" OR "eating habit" OR "dietary habit" OR "diet habit" OR "food habit" OR [mh "Feeding Behavior"] OR "feeding behavior" OR "beverage consumption" OR "beverage habit" OR "beverage intake*" OR "dietary profile*" OR "food profile" OR "diet profile*" OR "eating profile*" OR "dietary guideline" OR "dietary recommendation" OR "dietary intake" OR "food intake" OR "food consumption" OR "dietary consumption" OR "eating frequenc" OR "food frequenc" OR "eating style*" OR "dietary change *" OR "dietary choice" OR "food choice *" OR [mh "Diet, Mediterranean"] OR "Mediterranean Diet*" OR [mh "Dietary Approaches To Stop Hypertension"] OR "Dietary Approaches To Stop Hypertension Diet"” OR "DASH diet"” OR [mh "Diet, Gluten-Free"] OR "Gluten Free diet*" OR "prudent diet*” OR [mh "Diet, Paleolithic"] OR 
"Paleolithic Diet"” OR [mh "Diet, Vegetarian"] OR "vegetarian diet" OR "vegan diet" OR [mh "Healthy Diet"] OR "plant based diet"” OR [mh "Diet, Western"] OR "western diet"” OR [mh "Diet, Carbohydrate-Restricted"] OR "low-carbohydrate diet*" OR "high carbohydrate diet"” OR "Ketogenic Diet*” OR "Nordic Diet" OR [mh "Diet, Fat-Restricted"] OR [mh "Diet, High-Fat"] OR [mh "Diet, High-Protein"] OR "high protein diet*" OR "high-fat diet*" OR "low fat diet"” OR [mh "Diet, Protein-Restricted"] OR "low protein diet*" OR [mh "Diet, Sodium-Restricted"] OR "low-sodium diet*" OR "low salt diet*"OR (([mh "Dietary Proteins"] OR "dietary protein*” OR [mh "Dietary Carbohydrates"] OR "dietary carbohydrate*" OR [mh "Dietary Fats"] OR "dietary fat"” OR hypocaloric OR hypo-caloric) NEAR (diet OR diets OR consumption OR intake OR supplement*)) OR ("guideline adherence") NEAR (diet OR dietary OR food OR beverage)) OR ("diet score" OR "diet scores" OR "diet quality score" OR "diet quality scores" OR "diet quality index" OR "dietary habits score" OR kidmed OR "diet index" OR "dietary index" OR "Foodbased Index" OR "diet quality index" OR "food index" OR "food score" OR "food scores" OR "Mediterranean diet score" OR MedDietScore OR "healthy eating index" OR "food frequency questionnaire" OR "food frequency questionnaires" OR "food frequency survey" OR "food frequency surveys" OR [mh "Nutrition Surveys"] OR "nutrition survey" OR "nutrition surveys" OR "diet survey" OR "diet surveys" OR "food survey" OR "food surveys" OR "dietary questionnaire"):ti,ab,kw OR ((pattern OR patterns OR consumption OR habit*) NEAR ([mh ${ }^{\wedge}$ Diet] OR diet OR diets OR dietary OR [mh Food] OR food OR foods OR [mh Beverages] OR beverage OR beverages)):ti,ab,kw

\#2 - [mh "Pregnancy"] OR [mh "Pregnancy Complications"] OR [mh "Prenatal Exposure Delayed Effects"] OR [mh "Maternal Exposure"] OR [mh "Pregnant Women"] OR [mh "Mothers"] OR [mh "Peripartum Period"] OR [mh "Maternal Nutritional Physiological Phenomena"] OR [mh "Postpartum Period"] OR [mh Lactation] OR [mh "Breast Feeding"] OR [mh "Milk, Human"] OR (pregnancy OR pre-pregnancy OR prenatal OR pre-natal OR maternal OR mother OR mothers OR postpartum OR perinatal OR peri-natal OR pre-conception OR preconception OR peri-conception OR periconception OR peripartum OR peri-partum OR gestation* OR natal OR antenatal OR ante-natal OR puerperium OR postpartum OR postpartum OR perinatal OR peri-natal OR puerperium OR postpartal OR post-partal OR postnatal OR "post delivery" OR "after birth" OR lactation OR lactating OR breastfeeding OR breastfeeding OR breast feed* OR breast-feed* OR breastfed OR breast-fed OR breastfeed OR "human milk" OR "nursing women"):ti,ab,kw

\#3 - (weight NEAR/4 (reduction OR reduced OR reducing OR loss OR losses OR maintenanc* OR maintain* OR decreas ${ }^{*}$ OR watch OR control ${ }^{*}$ OR change* OR gain)):ti,ab,kw OR ("gestational weight gain" OR "weight gain" OR obesity OR obese OR overweight OR "body size" OR overnutrition OR adipos* OR anthropometry OR anthropometric* OR adipose OR "body weight" OR "body fat" OR weight OR "body mass index" OR BMI OR "weight status" OR "healthy weight" OR "body fat mass" OR "weight change" OR "weight changes" OR "weight loss*" OR "weight reduct" OR "body weight" OR "weight maint" OR "diet reduc" OR "weight cycling" OR "weight decreas" OR "weight watch*" OR "weight control*" OR "weight retention"):ti,ab,kw OR [mh "Gestational Weight Gain"] OR [mh ^"Weight Gain"] OR [mh Obesity] OR [mh "Body Size"] OR [mh ^Overnutrition] OR [mh Adiposity] OR [mh "body weight"] OR [mh "Body Composition"] OR [mh "Body Mass Index"] OR [mh "Adipose Tissue"] OR [mh "Weight Loss"] OR [mh "Weight Reduction Programs"] OR [mh "Body-Weight Trajectory"] OR [mh "Diet, Reducing"]

\#4 - \#1 AND \#2 AND \#3

Filters: publication year from 2000 to 2019 , Trials 


\section{Embase}

- Provider: Elsevier

- Date(s) searched: June 26, 2019, Update: November 7, 2019

- Date range searched: January 1, 2000 - June 26, 2019; Update: January 1, 2000 November 7, 2019

- Search terms:

\#1 - 'feeding behavior'/exp OR 'mediterranean diet'/de OR 'dash diet'/de OR 'gluten free diet'/exp OR 'paleolithic diet'/de OR 'vegetarian diet'/exp OR 'healthy diet'/de OR 'western diet'/de OR 'low carbohydrate diet'/exp OR 'low fat diet'/de OR 'lipid diet'/exp OR 'protein diet'/exp OR 'protein restriction'/de OR 'sodium restriction'/de OR 'dietary pattern*':ab,ti OR 'diet pattern*':ab,ti OR 'eating pattern': ab,ti OR 'food pattern': ab,ti OR 'diet quality':ab,ti OR 'eating habit': ab,ti OR 'dietary habit': ab,ti OR 'diet habit': ab,ti OR 'food habit"':ab,ti OR 'feeding behavior"':ab,ti OR 'beverage consumption':ab,ti OR 'beverage habit': ab,ti OR 'beverage intake $e^{* \prime}: a b, t i$ OR 'dietary profile ${ }^{\star \prime}: a b, t i$ OR 'food profile':ab,ti OR 'diet profile*':ab,ti OR 'eating profile ${ }^{* \prime}: a b, t i$ OR 'dietary guideline ${ }^{* \prime}: a b$, ti OR 'dietary recommendation':ab,ti OR 'dietary intake':ab,ti OR 'food intake':ab,ti OR 'food consumption':ab,ti OR 'dietary consumption':ab,ti OR 'eating frequenc ${ }^{*}: a b, t i$ OR 'food frequenc ${ }^{\star \prime}: a b$, ti OR 'eating style ${ }^{\star \prime}: a b, t i$ OR 'dietary change ${ }^{\star \prime}: a b, t i$ OR 'dietary choice ${ }^{* \prime}: a b, t i$ OR 'food choice ${ }^{\star \prime}: a b$, ti OR 'mediterranean $\operatorname{diet}^{\star \prime}:$ ab,ti OR 'dietary approaches to stop hypertension diet $^{\star \prime}: a b, t i$ OR 'dash diet ${ }^{\star \prime}: a b, t i$ OR

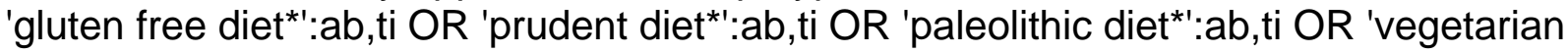

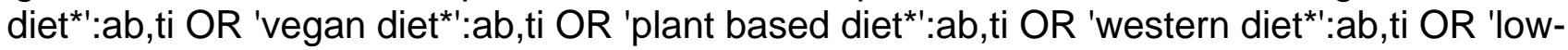
carbohydrate diet $^{* \prime}: a b, t i$ OR 'high carbohydrate diet $^{* \prime}: a b, t i$ OR 'ketogenic diet $^{* \prime}: a b, t i$ OR 'nordic diet $^{\star \prime}: a b, t i$ OR 'high protein diet*':ab,ti OR 'high-fat diet"':ab,ti OR 'low fat diet"':ab,ti OR 'low

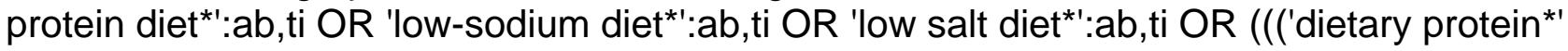
OR 'dietary carbohydrate*' OR 'dietary fat*' OR hypocaloric OR 'hypo caloric') NEAR/6 (diet OR diets OR consumption OR intake OR supplement $\left.\left.{ }^{\star}\right)\right)$ :ab,ti) ${ }^{*}$ OR (('guideline adherence' NEAR/6 (diet OR dietary OR food OR beverage)):ab,ti) OR 'diet score*':ab,ti OR 'diet quality score*':ab,ti OR 'dietary habits score*':ab,ti OR kidmed:ab,ti OR 'diet index':ab,ti OR 'dietary index*':ab,ti OR 'food-based index*':ab,ti OR 'diet quality index':ab,ti OR 'food index"':ab,ti OR 'food score ${ }^{* \prime}: a b, t i$ OR 'mediterranean diet score ${ }^{* 1}: a b, t i$ OR meddietscore:ab,ti OR 'healthy eating index':ab,ti OR 'food frequency questionnaire ${ }^{* 1}: a b, t i$ OR 'food frequency survey"':ab,ti OR 'nutrition survey*':ab,ti OR 'diet survey*':ab,ti OR 'food survey': ab,ti OR 'dietary questionnaire':ab,ti OR ((pattern OR patterns OR consumption OR habit $\left.{ }^{\star}\right)$ NEAR/6 (diet OR diets OR dietary OR food OR foods OR beverage OR beverages)):ab,ti)

\#2 - pregnancy:ab,ti OR 'pre pregnancy':ab,ti OR prenatal:ab,ti OR 'pre natal':ab,ti OR maternal:ab,ti OR mother:ab,ti OR mothers:ab,ti OR 'pre conception':ab,ti OR preconception:ab,ti OR 'peri conception':ab,ti OR periconception:ab,ti OR peripartum:ab,ti OR 'peri partum':ab,ti OR gestation*:ab,ti OR natal:ab,ti OR antenatal:ab,ti OR 'ante natal':ab,ti OR postpartum:ab,ti OR post-partum:ab,ti OR perinatal:ab,ti OR 'peri natal':ab,ti OR puerperium:ab,ti OR postpartal:ab,ti OR post-partal:ab,ti OR postnatal:ab,ti OR 'post delivery':ab,ti OR 'after birth':ab,ti OR lactation:ab,ti OR lactating:ab,ti OR breastfeeding:ab,ti OR breast-feeding:ab,ti OR 'breast feed"':ab,ti OR breastfed:ab,ti OR 'breast fed':ab,ti OR breastfeed:ab,ti OR 'human milk':ab,ti OR 'nursing women':ab,ti OR 'pregnancy'/exp/mj OR 'pregnancy complication'/exp/mj OR 'prenatal exposure'/mj OR 'maternal exposure'/mj OR 'pregnant woman'/mj OR 'mother'/mj OR 'puerperium'/exp/mj OR 'maternal nutrition'/mj OR 'lactation'/mj OR 'breast feeding'/exp/mj OR 'breast milk'/exp/mj 
\#3 - ((weight NEAR/4 (reduction OR reduced OR reducing OR loss OR losses OR maintenanc ${ }^{*}$ OR maintain* OR decreas* OR watch OR control* OR change* OR gain)):ab,ti) OR 'gestational weight gain':ab,ti OR 'weight gain':ab,ti OR obesity:ab,ti OR obese:ab,ti OR overweight:ab,ti OR 'body size':ab,ti OR overnutrition:ab,ti OR adipos*:ab,ti OR anthropometry:ab,ti OR anthropometric*:ab,ti OR adipose:ab,ti OR 'body fat':ab,ti OR weight:ab,ti OR 'body mass index':ab,ti OR bmi:ab,ti OR 'weight status':ab,ti OR 'healthy weight':ab,ti OR 'body fat mass':ab,ti OR 'weight change':ab,ti OR 'weight changes':ab,ti OR 'weight loss"':ab,ti OR 'weight reduct"':ab,ti OR 'body weight':ab,ti OR 'weight maint"':ab,ti OR 'diet reduc*':ab,ti OR 'weight cycling':ab,ti OR 'weight decreas ${ }^{* \prime}: a b, t i$ OR 'weight watch':ab,ti OR 'weight control*':ab,ti OR 'weight retention':ab,ti OR 'gestational weight gain'/mj OR 'body weight gain'/de OR 'obesity'/exp/mj OR 'body size'/mj OR 'overnutrition'/mj OR 'body weight'/exp/mj OR 'body composition'/exp/mj OR 'body mass'/de OR 'adipose tissue'/exp/mj OR 'body weight loss'/exp/mj OR 'weight loss program'/mj OR 'weight trajectory (body weight)'/mj OR 'low calorie diet'/exp/mj

\section{\#4 - \#1 AND \#2 AND \#3}

\#5 - \#4 AND ([article]/lim OR [article in press]/lim) AND [humans]/lim AND [english]/lim AND [2000-2019]/py NOT ([conference abstract]/lim OR [conference paper]/lim OR [editorial]/lim OR [erratum]/lim OR [letter]/lim OR [note]/lim OR [review]/lim OR [systematic review]/lim OR [meta analysis]/lim)

\section{Cumulative Index of Nursing and Allied Health Literature (CINAHL Plus)}

- Provider: EBSCOhost

- Date(s) searched: June 26, 2019; Update: November 7, 2019

- Date range searched: January 1, 2000 - June 26, 2019; Update: January 1, 2000 November 7, 2019

- Search terms:

\#1 - "dietary pattern"” OR "diet pattern" OR "eating pattern*" OR "food pattern" OR "diet quality" OR "eating habit" OR "dietary habit" OR "diet habit" OR "food habit" OR MH "Eating Behavior+" OR "feeding behavior" OR "beverage consumption" OR "beverage habit" OR "beverage intake*" OR "dietary profile*" OR "food profile*" OR "diet profile*" OR "eating profile" OR "dietary guideline*" OR "dietary recommendation*" OR "dietary intake*" OR "food intake" " OR "food consumption" OR "dietary consumption" OR "eating frequenc" OR "food frequenc" OR "eating style " OR "dietary change*" OR "dietary choice *" OR food choice" OR MH "Diet, Mediterranean" OR "Mediterranean Diet" OR MH "Dietary Approaches To Stop Hypertension" OR "Dietary Approaches To Stop Hypertension Diet"” OR "DASH diet"” OR MH "Diet, Gluten-Free" OR "Gluten Free diet"” OR "prudent diet"” OR MH "Diet, Paleolithic" OR "Paleolithic Diet"” OR MH "Diet, Vegetarian" OR "vegetarian diet" OR "vegan diet" OR MH "Healthy Diet" OR "plant based diet"” OR MH "Diet, Western" OR "western diet*” OR MH "Diet, Carbohydrate-Restricted" OR "low-carbohydrate diet" OR "high carbohydrate diet" OR "Ketogenic Diet"” OR "Nordic Diet"” OR MH "Diet, Fat-Restricted" OR MH "Diet, High-Fat" OR MH "Diet, High-Protein" OR "high protein diet" OR "high-fat diet"” OR "low fat diet" OR MH "Diet, Protein-Restricted" OR "low protein diet"” OR MH "Diet, Sodium-Restricted" OR "lowsodium diet*" OR "low salt diet"” OR ((MH "Dietary Proteins" OR "dietary protein"” OR MH "Dietary Carbohydrates" OR "dietary carbohydrate" OR MH "Dietary Fats" OR "dietary fat*" OR hypocaloric OR hypo-caloric) AND (diet OR diets OR consumption OR intake OR 
supplementation)) OR (MH "Guideline Adherence" AND (diet OR dietary OR food OR beverage)) OR ("diet score*" OR "diet quality score" OR "diet quality index" OR "dietary habits score *" OR kidmed OR "diet index" OR "dietary index" OR "Food-based Index" OR "diet quality index" OR "food index" OR "food score*" OR "Mediterranean diet score*" OR MedDietScore OR "healthy eating index" OR "food frequency questionnaire*" OR "food frequency survey" OR MH "Nutrition Surveys" OR "nutrition survey" OR "diet survey*" OR "food survey"” OR "dietary questionnaire ") OR ((pattern OR patterns OR consumption OR habit*) AND (MH "Diet" OR diet OR diets OR dietary OR MH "Food" OR food OR foods OR $\mathrm{MH}$ "Beverages" OR beverage OR beverages))

\#2 - postpartum OR post-partum OR MH "Postpartum Period" OR postpartal OR post-partal OR postnatal OR post-natal OR "post deliver" OR "after birth" OR MH pregnancy OR MH "pregnancy complications" OR MH "Prenatal Exposure Delayed Effects" OR MH "Maternal Exposure" OR MH "pregnant women" OR pregnan* OR pre-pregnancy OR prepregnancy OR prenatal OR antenatal OR maternal OR mother OR mothers OR perinatal OR peri-natal OR peri-conception OR periconception OR MH "Peripartum Period" OR peripartum OR peripartum OR gestation* OR natal OR puerperium OR MH "Maternal Nutritional Physiological Phenomena" OR MH "Breast Feeding"OR breastfeeding OR breast-feeding OR MH "Milk, Human" OR "human milk" OR MH Lactation OR lactation OR lactating OR breastfeeding OR "breast feed"” OR breast-feed* OR breastfed OR breast-fed OR breastfeed* OR "nursing women" OR "nursing mother"

\#3 - MH "Gestational Weight Gain" OR MH "Weight Gain" OR MH Obesity OR MH "Body Size" OR MH Overnutrition OR MH Adiposity OR MH "Body Weight" OR MH "Body Composition" OR MH "Body Mass Index" OR MH "Adipose Tissue" OR MH "Weight Loss" OR $\mathrm{MH}$ "Weight Reduction Programs" OR MH "Body-Weight Trajectory" OR MH "Diet, Reducing" OR gestational weight gain OR weight gain OR obesity OR obese OR overweight OR "body size" OR overnutrition OR adipos* OR anthropometry OR anthropometric* OR adipose OR "body weight" OR "body fat" OR weight OR "body mass index" OR BMI OR "weight status" OR "healthy weight" OR "body fat mass" OR "weight change" OR "weight changes" OR "weight loss*" OR "weight reduc*" OR "body weight" OR MH "Body-Weight Trajectory" OR "weight change" OR "weight maint" OR "diet reduc" OR "weight cycling" OR "weight decreas" OR "weight watch"” OR "weight control*" OR "weight retention" OR (weight N4 (reduction OR reduced OR reducing OR loss OR losses OR maintenanc* OR maintain* OR decreas* OR watch OR control* OR change* OR gain ))

\#4 - \#1 AND \#2 AND \#3

\#5 - \#4 NOT (MH "Literature Review" OR MH "Meta Analysis" OR MH "Systematic Review" OR MH "News" OR MH "Retracted Publication" OR MH "Retraction of Publication)

Filters: Published Date: 20000101 - 20190626; Update: Published Date: 20000101 20191107

\section{LITERATURE SEARCH AND SCREENING RESULTS}

The flow chart (Figure 2) below illustrates the literature search and screening results for articles examining the systematic review questions on dietary patterns and gestational weight gain (GWG), as well as dietary patterns and postpartum weight loss (PPWL). Articles on dietary patterns and GWG and dietary patterns and PPWL were searched for and screened 
together. This was done to leverage the overlap in topical areas and to improve efficiency. After the initial electronic database search (January 2000-June 2019), an updated search was conducted to also capture macronutrient distribution articles (January 2000-November 2019). The results of both electronic database searches, after removal of duplicates, were screened independently by two NESR analysts using a step-wise process by reviewing titles, abstracts, and full-texts to determine which articles met the inclusion criteria for each systematic review question depicted in the flow chart. Refer to Table 8 for the rationale for exclusion for each excluded full-text article. A manual search was done to find articles that were not identified when searching the electronic databases; all manually identified articles are also screened to determine whether they meet criteria for inclusion.

\section{Figure 2: Flow chart of literature search and screening results}

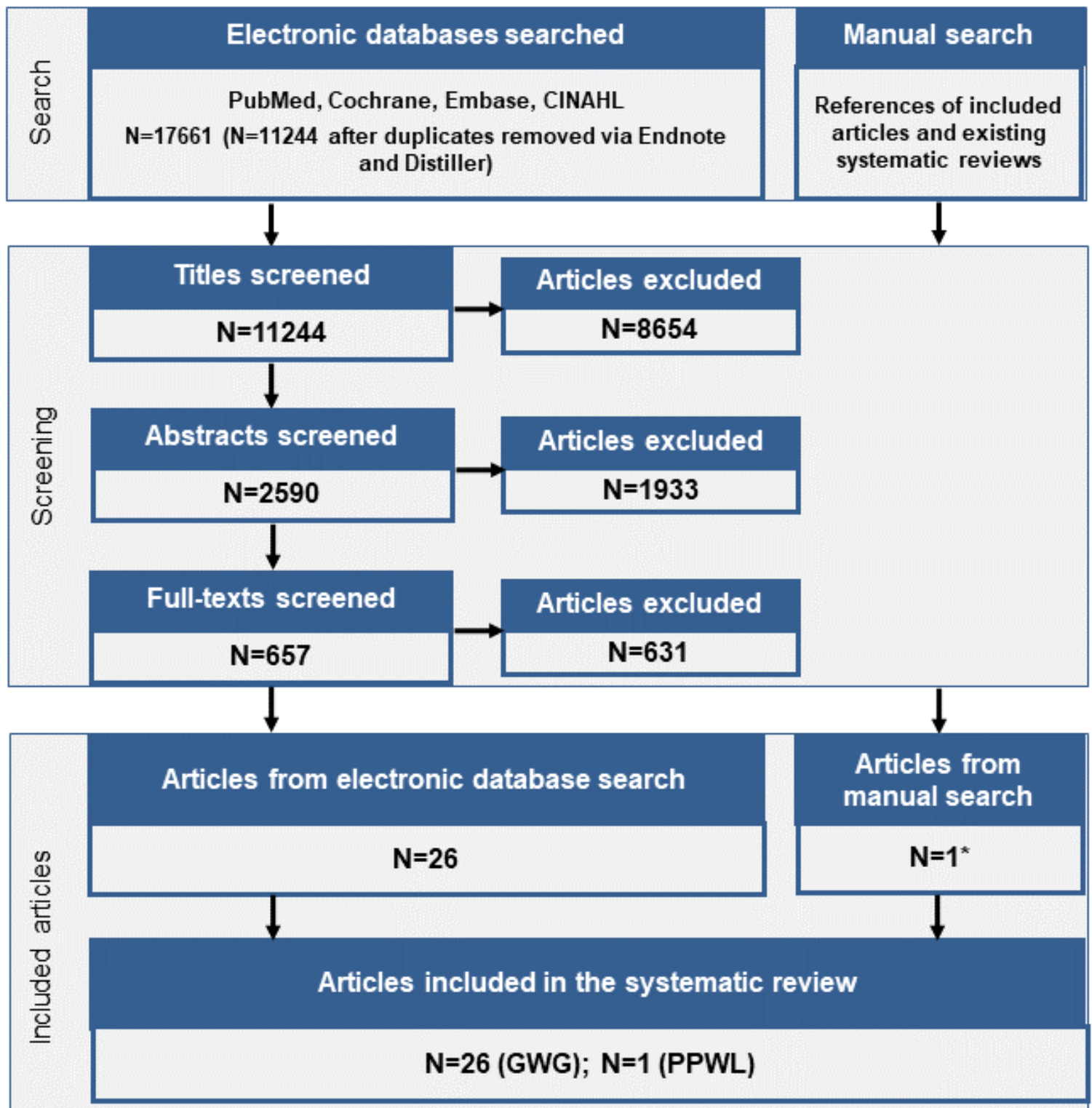

*The article identified from the manual search was included in the GWG systematic review 


\section{Excluded articles}

The table below lists the articles excluded after full-text screening, and includes columns for the categories of inclusion and exclusion criteria (see Table 7) that studies were excluded based on. At least one reason for exclusion is provided for each article, though this may not reflect all possible reasons for exclusion. Information about articles excluded after title and abstract screening is available upon request.

\section{Table 8. Articles excluded after full text screening with rationale for exclusion}

\section{Citation}

1. Aaltonen, J, Ojala, T, Laitinen, K, Poussa, T, Ozanne, S, Isolauri, E. Impact of maternal diet during pregnancy and breastfeeding on infant metabolic programming: a prospective randomized controlled study. Eur J Clin Nutr. 2011. 65:10-9. doi:10.1038/ejcn.2010.225.

2. Abdel-Aziz, SB, Hegazy, IS, Mohamed, DA, Abu El Kasem, MMA, Hagag, SS. Effect of dietary counseling on preventing excessive weight gain during pregnancy. Public Health. 2018. 154:172-181. doi:10.1016/j.puhe.2017.10.014.

3. Abreu, S, Santos, PC, Montenegro, N, Mota, J. Relationship between dairy product intake during pregnancy and neonatal and maternal outcomes among Portuguese women. Obes Res Clin Pract. 2017. 11:276-286. doi:10.1016/j.orcp.2016.07.001.

4. Adair, LS, Kuzawa, CW, Borja, J. Maternal energy stores and diet composition during pregnancy program adolescent blood pressure. Circulation. 2001. 104:1034-9. doi:10.1161/hc3401.095037.

5. Adherence to Canada's Food Guide Recommendations during Pregnancy: Nutritional Epidemiology and Public Health. Curr Dev Nutr. 2017. 1:e000356. doi:10.3945/cdn.116.000356.

6. Ainscough, K, Kennelly, MA, O'Sullivan, EJ, Lindsay, KL, Gibney, ER, McCarthy, M, McAuliffe, FM. Impact of a smartphone app supporting a lifestyle intervention in overweight and obese pregnancy on on maternal health and lifestyle outcomes. American journal of obstetrics and gynecology. 2018. 218:S598-S599.

7. Akbari, Z, Mansourian, M, Kelishadi, R. Relationship of the intake of different food groups by pregnant mothers with the birth weight and gestational age: Need for public and individual educational programs. J Educ Health Promot. 2015. 4:23. doi:10.4103/22779531.154109.

8. Allman, BR, Diaz Fuentes, E, Williams, DK, Turner, DE, Andres, A, Borsheim, E. Obesity Status Affects the Relationship Between Protein Intake and Insulin Sensitivity in Late Pregnancy. Nutrients. 2019. 11. doi:10.3390/nu11092190.

\section{Rationale}

Intervention/Exposure; Outcome

Intervention/Exposure

Intervention/Exposure

Outcome

Outcome

Abstract

Intervention/Exposure; Outcome

Intervention/Exposure; Outcome 
9. Althuizen, E, van Poppel, MN, Seidell, JC, van Mechelen, W. Correlates of absolute and excessive weight gain during pregnancy. J Womens Health (Larchmt). 2009. 18:1559-66. doi:10.1089/jwh.2008.1275.

10. Alves-Santos, NH, Cocate, PG, Benaim, C, Farias, DR, Emmett, PM, Kac, G. Prepregnancy Dietary Patterns and Their Association with Perinatal Outcomes: A Prospective Cohort Study. J Acad Nutr Diet. 2019. doi:10.1016/j.jand.2019.02.016.

\section{Outcome}

Alves-Santos, NH, Cocate, PG, Eshriqui, I, Benaim, C, Barros, EG, Emmett, PM, Kac, G. Dietary patterns and their association with adiponectin and leptin concentrations throughout pregnancy: a prospective cohort. Br J Nutr. 2018. 119:320-329. doi:10.1017/s0007114517003580.

12. Alves-Santos, NH, Eshriqui, I, Franco-Sena, AB, Cocate, PG, Freitas-Vilela, AA, Benaim, C, Vaz Jdos, S, Castro, MB, Kac, G. Dietary intake variations from pre-conception to gestational period according to the degree of industrial processing: A Brazilian cohort. Appetite. 2016. 105:164-71. doi:10.1016/j.appet.2016.05.027.

13. Amezcua-Prieto, C, Martínez-Galiano, JM, Cano-Ibáñez, N, Olmedo-Requena, R, BuenoCavanillas, A, Delgado-Rodríguez, M. Types of carbohydrates intake during pregnancy and frequency of a small for gestational age newborn: A case-control study. Nutrients. 2019. 11. doi:10.3390/nu11030523.

14. Anand, SS, Gupta, M, Teo, KK, Schulze, KM, Desai, D, Abdalla, N, Zulyniak, M, de Souza, Intervention/Exposure; Outcome R, Wahi, G, Shaikh, M, Beyene, J, de Villa, E, Morrison, K, McDonald, SD, Gerstein, H. Causes and consequences of gestational diabetes in South Asians living in Canada: results from a prospective cohort study. CMAJ Open. 2017. 5:E604-e611. doi:10.9778/cmajo.20170027.

15. Anleu, E, Reyes, M, Araya, BM, Flores, M, Uauy, R, Garmendia, ML. Effectiveness of an Intervention/Exposure Intervention of Dietary Counseling for Overweight and Obese Pregnant Women in the Consumption of Sugars and Energy. Nutrients. 2019. 11. doi:10.3390/nu11020385.

16. Antal, M. Nutritional status of Hungarian pregnant women. Forum Nutr. 2003. 56:229-31. Intervention/Exposure; Outcome

17. Antonakou, A, Papoutsis, D, Panou, I, Chiou, A, Matalas, AL. Role of exclusive Intervention/Exposure breastfeeding in energy balance and weight loss during the first six months postpartum. Clin Exp Obstet Gynecol. 2013. 40:485-8.

18. Apolzan, JW, Myers, CA, Cowley, AD, Brady, H, Hsia, DS, Stewart, TM, Redman, LM, Martin, CK. Examination of the reliability and validity of the Mindful Eating Questionnaire in pregnant women. Appetite. 2016. 100:142-51. doi:10.1016/j.appet.2016.02.025.

19. Arredondo, A, Torres, C, Orozco, E, Pacheco, S, Huang, F, Zambrano, E, BolanosJimenez, F. Socio-economic indicators, dietary patterns, and physical activity as determinants of maternal obesity in middle-income countries: Evidences from a cohort study in Mexico. Int J Health Plann Manage. 2019. 34:e713-e725. doi:10.1002/hpm.2684. 
20. Artal, R, Catanzaro, RB, Gavard, JA, Mostello, DJ, Friganza, JC. A lifestyle intervention of weight-gain restriction: diet and exercise in obese women with gestational diabetes mellitus. Appl Physiol Nutr Metab. 2007. 32:596-601. doi:10.1139/h07-024.

21. Arvizu, M, Afeiche, MC, Hansen, S, Halldorsson, TF, Olsen, SF, Chavarro, JE. Fat intake

Outcome

during pregnancy and risk of preeclampsia: a prospective cohort study in Denmark. European Journal of Clinical Nutrition. 2019. 73:1040-1048. doi:10.1038/s41430-0180290-z.

22. Arvizu, M, Stuart, J, Rich-Edwards, J, Gaskins, A, Rosner, B, Chavarro, J. Adherence to Pre-pregnancy DASH Dietary Pattern and Diet Recommendations from the American Heart Association and the Risk of Preeclampsia (OR35-06-19). Curr Dev Nutr. 2019. 3. doi:10.1093/cdn/nzz048.OR35-06-19.

23. Asbjornsdottir, B, Ronneby, H, Vestgaard, M, Ringholm, L, Nichum, VL, Jensen, DM, Raben, A, Damm, P, Mathiesen, ER. Lower daily carbohydrate consumption than recommended by the Institute of Medicine is common among women with type 2 diabetes in early pregnancy in Denmark. Diabetes Res Clin Pract. 2019. 152:88-95. doi:10.1016/j.diabres.2019.05.012.

24. Asci, O, Rathfisch, G. Effect of lifestyle interventions of pregnant women on their dietary habits, lifestyle behaviors, and weight gain: a randomized controlled trial. J Health Popul Nutr. 2016. 35:7. doi:10.1186/s41043-016-0044-2.

25. Asemi, Z, Samimi, M, Tabassi, Z, Esmaillzadeh, A. The effect of DASH diet on pregnancy Health Status outcomes in gestational diabetes: a randomized controlled clinical trial. Eur J Clin Nutr. 2014. 68:490-5. doi:10.1038/ejcn.2013.296.

26. Asemi, Z, Samimi, M, Tabassi, Z, Sabihi, S, Esmaillzadeh, A. A randomized controlled clinical trial investigating the effect of DASH diet on insulin resistance, inflammation, and oxidative stress in gestational diabetes. Nutrition. 2013. 29:619-624. doi:10.1016/j.nut.2012.11.020.

27. Ashman, AM, Collins, CE, Hure, AJ, Jensen, M, Oldmeadow, C. Maternal diet during early childhood, but not pregnancy, predicts diet quality and fruit and vegetable acceptance in offspring. Matern Child Nutr. 2016. 12:579-90. doi:10.1111/mcn.12151.

28. Ashman, AM, Collins, CE, Weatherall, L, Brown, LJ, Rollo, ME, Clausen, D, Blackwell, CC, Pringle, KG, Attia, J, Smith, R, Lumbers, ER, Rae, KM. A cohort of Indigenous Australian women and their children through pregnancy and beyond: the Gomeroi gaaynggal study. J Dev Orig Health Dis. 2016. 7:357-68. doi:10.1017/s204017441600009x.

\section{Abstract}

Intervention/Exposure; Health Status

Intervention/Exposure

Health Status

Outcome

Intervention/Exposure 
29. Assaf-Balut, C, de la Torre, NG, Fuentes, M, Durán, A, Bordiú, E, Del Valle, L, Valerio, J, Jiménez, I, Herraiz, MA, Izquierdo, N, Torrejón, MJ, de Miguel, MP, Barabash, A, Cuesta, M, Rubio, MA, Calle-Pascual, AL. A high adherence to six food targets of the mediterranean diet in the late first trimester is associated with a reduction in the risk of materno-foetal outcomes: The st. carlos gestational diabetes mellitus prevention study. Nutrients. 2019. 11. doi:10.3390/nu11010066.

30. Assaf-Balut, C, Garcia de la Torre, N, Duran, A, Fuentes, M, Bordiu, E, Del Valle, L, Valerio, J, Familiar, C, Jimenez, I, Herraiz, MA, Izquierdo, N, Torrejon, MJ, Runkle, I, de Miguel, MP, Moraga, I, Montanez, MC, Barabash, A, Cuesta, M, Rubio, MA, CallePascual, AL. Medical nutrition therapy for gestational diabetes mellitus based on Mediterranean Diet principles: a subanalysis of the St Carlos GDM Prevention Study. BMJ Open Diabetes Res Care. 2018. 6:e000550. doi:10.1136/bmjdrc-2018-000550.

31. Aydin, EK, Ozturk, S. Assessment of the Diets and Weights of Primiparous and Multiparous Pregnant Women in the Last Trimester. International Journal of Caring Sciences. 2016. 9:1033-1039.

32. Babaei, M, Banaem, LM. Nutritional status of pregnant women and urine calcium-tocreatinine ratio during 24th-28th weeks of pregnancy and their relationship with the incidence of hypertensive disorders during pregnancy. Journal of Kermanshah University of Medical Sciences. 2018. 22. doi:10.5812/jkums.69638.

33. Badon, SE, Miller, RS, Qu, C, Sorensen, TK, Williams, MA, Enquobahrie, DA. Maternal healthy lifestyle during early pregnancy and offspring birthweight: differences by offspring sex. J Matern Fetal Neonatal Med. 2018. 31:1111-1117. doi:10.1080/14767058.2017.1309383.

34. Bao, W, Bowers, K, Tobias, DK, Olsen, SF, Chavarro, J, Vaag, A, Kiely, M, Zhang, C. Prepregnancy low-carbohydrate dietary pattern and risk of gestational diabetes mellitus: a prospective cohort study. Am J Clin Nutr. 2014. 99:1378-84. doi:10.3945/ajcn.113.082966.

35. Bao, W. Comment on Koivusalo et al. Gestational diabetes mellitus can be prevented by lifestyle intervention: the finnish gestational diabetes prevention study (RADIEL): a randomized controlled Trial. Diabetes Care 2016; 39: 24-30. Diabetes Care. 39 (8) (pp e125), 2016. doi:10.2337/dc16-0665.

36. Bao, W, Tobias, DK, Hu, FB, Chavarro, J, Zhang, C. Pre-pregnancy potato consumption and risk of gestational diabetes mellitus. BMJ: British Medical Journal. 2016. 352:h6898h6898.

37. Barbieri, MA, Portella, AK, Silveira, PP, Bettiol, H, Agranonik, M, Silva, AA, Goldani, MZ. Severe intrauterine growth restriction is associated with higher spontaneous carbohydrate intake in young women. Pediatr Res. 2009. 65:215-20. doi:10.1203/PDR.0b013e31818d6850. 
38. Barebring, L, Brembeck, P, Lof, M, Brekke, HK, Winkvist, A, Augustin, H. Food intake and gestational weight gain in Swedish women. Springerplus. 2016. 5:377. doi:10.1186/s40064-016-2015-x.

39. Barnes, RA, Edghill, N, Mackenzie, J, Holters, G, Ross, GP, Jalaludin, BB, Flack, JR. Predictors of large and small for gestational age birthweight in offspring of women with Intervention/Exposure; Outcome gestational diabetes mellitus. Diabetic Medicine. 2013. 30:1040-1046. doi:10.1111/dme.12207.

40. Bawadi, HA, Al-Kuran, O, Al-Bastoni, LA, Tayyem, RF, Jaradat, A, Tuuri, G, Al-Beitawi, SN, Al-Mehaisen, LM. Gestational nutrition improves outcomes of vaginal deliveries in Jordan: an epidemiologic screening. Nutr Res. 2010. 30:110-7. doi:10.1016/j.nutres.2010.01.005.

41. Baykan, A, Yalcin, SS, Yurdakok, K. Does maternal iron supplementation during the lactation period affect iron status of exclusively breast-fed infants? Turk J Pediatr. 2006. 48:301-7.

42. Beardsall, A, Perreault, M, Farncombe, T, Vanniyasingam, T, Thabane, L, Teo, KK, Atkinson, SA. Maternal and child factors associated with bone length traits in children at 3years of age. Bone. 2019. 127:1-8. doi:10.1016/j.bone.2019.05.025.

43. Bechtel-Blackwell, DA. Computer-assisted self-interview and nutrition education in pregnant teens. Clin Nurs Res. 2002. 11:450-62. doi:10.1177/105477302237456.

44. Belan, M, Carranza-Mamane, B, AinMelk, Y, Pesant, MH, Duval, K, Jean-Denis, F, Langlois, MF, Lavoie, H, Waddell, G, Baillargeon, JP. A lifestyle intervention targeting women with obesity and infertility improves their fertility outcomes, especially in women with PCOS: a randomized controlled trial. Fertility and sterility. 2019. 112:e40-. doi:10.1016/j.fertnstert.2019.07.234.

45. Ben Naftali, Y, Chermesh, I, Solt, I, Friedrich, Y, Lowenstein, L. Achieving the Recommended Gestational Weight Gain in High-Risk Versus Low-Risk Pregnancies. Isr Med Assoc J. 2018. 20:411-414.

46. Benaim, C, Freitas-Vilela, AA, Pinto, TJP, Lepsch, J, Farias, DR, Dos Santos Vaz, J, EIBacha, T, Kac, G. Early pregnancy body mass index modifies the association of prepregnancy dietary patterns with serum polyunsaturated fatty acid concentrations throughout pregnancy in Brazilian women. Matern Child Nutr. 2018. 14. doi: $10.1111 / \mathrm{mcn} .12480$.

47. Bennett, WL, Liu, SH, Yeh, HC, Nicholson, WK, Gunderson, EP, Lewis, CE, Clark, JM. Changes in weight and health behaviors after pregnancies complicated by gestational diabetes mellitus: the CARDIA study. Obesity (Silver Spring). 2013. 21:1269-75. doi:10.1002/oby.20133. 
48. Berkey, CS, Tamimi, RM, Willett, WC, Rosner, B, Hickey, M, Toriola, AT, Lindsay Frazier, A, Colditz, GA. Dietary intake from birth through adolescence in relation to risk of benign breast disease in young women. Breast Cancer Res Treat. 2019. doi:10.1007/s10549019-05323-8.

49. Bertolotto, A, Volpe, L, Calianno, A, Pugliese, MC, Lencioni, C, Resi, V, Ghio, A, Corfini, M, Benzi, L, Del Prato, S, Di Cianni, G. Physical activity and dietary habits during pregnancy: effects on glucose tolerance. J Matern Fetal Neonatal Med. 2010. 23:1310-4. doi:10.3109/14767051003678150.

50. Bertz, F, Winkvist, A, Brekke, HK. Sustainable weight loss among overweight and obese lactating women is achieved with an energy-reduced diet in line with dietary recommendations: results from the LEVA randomized controlled trial. J Acad Nutr Diet. 2015. 115:78-86. doi:10.1016/j.jand.2014.05.017.

51. Black, MM, Papas, MA, Bentley, ME, Cureton, P, Saunders, A, Le, K, Anliker, J, Robinson, $\mathrm{N}$. Overweight adolescent African-American mothers gain weight in spite of intentions to lose weight. J Am Diet Assoc. 2006. 106:80-7. doi:10.1016/j.jada.2005.09.049.

52. Blumfield, ML, Hure, AJ, MacDonald-Wicks, LK, Smith, R, Simpson, SJ, Giles, WB, Raubenheimer, D, Collins, CE. Dietary balance during pregnancy is associated with fetal adiposity and fat distribution. Am J Clin Nutr. 2012. 96:1032-41. doi:10.3945/ajcn.111.033241.

53. Blumfield, ML, Schreurs, M, Rollo, ME, MacDonald-Wicks, LK, Kokavec, A, Collins, CE. The association between portion size, nutrient intake and gestational weight gain: a secondary analysis in the WATCH study 2006/7. J Hum Nutr Diet. 2016. 29:271-80. doi:10.1111/jhn.12330.

54. Bo, S, Menato, G, Lezo, A, Signorile, A, Bardelli, C, De Michieli, F, Massobrio, M, Pagano, Intervention/Exposure; Outcome G. Dietary fat and gestational hyperglycaemia. Diabetologia. 2001. 44:972-8. doi:10.1007/s001250100590.

55. Bobinski, R, Mikulska, M, Mojska, H, Ulman-Wodarz, I, Sodowska, P. Assessment of the diet components of pregnant women as predictors of risk of preterm birth and born baby with low birth weight. Ginekol Pol. 2015. 86:292-9.

56. Boghossian, NS, Yeung, EH, Lipsky, LM, Poon, AK, Albert, PS. Dietary patterns in association with postpartum weight retention. Am J Clin Nutr. 2013. 97:1338-45. doi:10.3945/ajcn.112.048702.

57. Bonakdar, SA, Dorosty Motlagh, AR, Bagherniya, M, Ranjbar, G, DaryabeygiKhotbehsara, R, Mohajeri, SAR, Safarian, M. Pre-pregnancy Body Mass Index and Maternal Nutrition in Relation to Infant Birth Size. Clin Nutr Res. 2019. 8:129-137. doi:10.7762/cnr.2019.8.2.129.

Population; Outcome

Intervention/Exposure; No association between diet and GWG examined

\section{Outcome}

Population

Outcome; Comparator

Intervention/Exposure; Outcome

Intervention/Exposure; Outcome

Population

Study Design; Outcome 
58. Bouwland-Both, MI, Steegers-Theunissen, RP, Vujkovic, M, Lesaffre, EM, Mook-

Kanamori, DO, Hofman, A, Lindemans, J, Russcher, H, Jaddoe, VW, Steegers, EA. A periconceptional energy-rich dietary pattern is associated with early fetal growth: the Generation R study. Bjog. 2013. 120:435-45. doi:10.1111/1471-0528.12086.

59. Brandhagen, M, Lissner, L, Brantsaeter, AL, Meltzer, HM, Haggkvist, AP, Haugen, M, Intervention/Exposure

Winkvist, A. Breast-feeding in relation to weight retention up to 36 months postpartum in the Norwegian Mother and Child Cohort Study: modification by socio-economic status? Public Health Nutr. 2014. 17:1514-23. doi:10.1017/s1368980013001869.

60. Brantsæter, AL, Haugen, M, Myhre, R, Sengpiel, V, Englund-Ögge, L, Nilsen, RM, Borgen, Study Design

I, Duarte-Salles, T, Papadopoulou, E, Vejrup, K, Von Ruesten, A, Hillesund, ER,

Birgisdottir, BE, Magnus, P, Trogstad, L, Jacobsson, B, Bacelis, J, Myking, S, Knutsen, HK, Kvalem, HE, Alexander, J, Mendez, M, Meltzer, HM. Diet matters, particularly in pregnancy - Results from MoBa studies of maternal diet and pregnancy outcomes. Norsk Epidemiologi. 2014. 24:63-77.

61. Brekke, HK, Bertz, F, Rasmussen, KM, Bosaeus, I, Ellegård, L, Winkvist, A. Diet and exercise interventions among overweight and obese lactating women: Randomized trial of effects on cardiovascular risk factors. PLoS ONE. 2014. 9. doi:10.1371/journal.pone.0088250.

62. Briley, C, Flanagan, NL, Lewis, NM. In-home prenatal nutrition intervention increased dietary iron intakes and reduced low birthweight in low-income African-American women. Journal of the American Dietetic Association. 2002. 102:984-987.

63. Broekhuizen, K, Simmons, D, Devlieger, R, van Assche, A, Jans, G, Galjaard, S, Corcoy, R, Adelantado, JM, Dunne, F, Desoye, G, Harreiter, J, Kautzky-Willer, A, Damm, P, Mathiesen, ER, Jensen, DM, Andersen, LL, Lapolla, A, Dalfra, MG, Bertolotto, A, WenderOzegowska, E, Zawiejska, A, Hill, D, Snoek, FJ, Jelsma, JGM, Bosmans, JE, van Poppel, MNM, van Dongen, JM. Cost-effectiveness of healthy eating and/or physical activity promotion in pregnant women at increased risk of gestational diabetes mellitus: economic evaluation alongside the DALI study, a European multicenter randomized controlled trial. Int J Behav Nutr Phys Act. 2018. 15:23. doi:10.1186/s12966-018-0643-y.

64. Brustman, LE, Langer, O, Bimson, B, Scarpelli, S, El Daouk, M. Weight gain in gestational diabetes: the effect of treatment modality. J Matern Fetal Neonatal Med. 2016. 29:1025-9. doi:10.3109/14767058.2015.1034101.

65. Buckingham-Schutt, LM, Ellingson, LD, Vazou, S, Campbell, CG. The Behavioral Wellness in Pregnancy study: a randomized controlled trial of a multi-component intervention to promote appropriate weight gain. The American journal of clinical nutrition. 2019. 109:1071-1079. doi:10.1093/ajcn/nqy359. 
66. Buhling, KJ, Elsner, E, Wolf, C, Harder, T, Engel, B, Wascher, C, Siebert, G,

Dudenhausen, JW. No influence of high- and low-carbohydrate diet on the oral glucose tolerance test in pregnancy. Clin Biochem. 2004. 37:323-7. doi:10.1016/j.clinbiochem.2003.11.008.

67. Bzikowska-Jura, A, Czerwonogrodzka-Senczyna, A, Oledzka, G, Szostak-Wegierek, D, Intervention/Exposure Weker, $\mathrm{H}$, Wesolowska, A. Maternal Nutrition and Body Composition During Breastfeeding: Association with Human Milk Composition. Nutrients. 2018. 10. doi:10.3390/nu10101379.

68. Cahill, JM, Freeland-Graves, JH, Shah, BS, Lu, H, Pepper, MR. Determinants of weight loss after an intervention in low-income women in early postpartum. J Am Coll Nutr. 2012. 31:133-43.

69. Caire-Juvera, G, Casanueva, E, Bolanos-Villar, AV, de Regil, LM, Calderon de la Barca, AM. No changes in weight and body fat in lactating adolescent and adult women from Mexico. Am J Hum Biol. 2012. 24:425-31. doi:10.1002/ajhb.22234.

70. Cano Ibanez, N, Martinez Galiano, JM, Amezcua Prieto, C, Olmedo Requena, R, Bueno Cavanillas, A, Delgado Rodriguez, M. Meat and meat products intake in pregnancy and risk of small for gestational age infants. A case-control study. Nutr Hosp. 2019. 36:405411. doi:10.20960/nh.2366.

71. Cao, LL, Yan, CH, Yu, XD, Tian, Y, Zhao, L, Liu, JX, Shen, XM. Relationship between serum concentrations of polychlorinated biphenyls and organochlorine pesticides and dietary habits of pregnant women in Shanghai. Sci Total Environ. 2011. 409:2997-3002. doi:10.1016/j.scitotenv.2011.04.040.

72. Carmichael, SL, Ma, C, Feldkamp, ML, Shaw, GM. Comparing Usual Dietary Intakes Among Subgroups of Mothers in the Year Before Pregnancy. Public Health Rep. 2019. 134:155-163. doi:10.1177/0033354918821078.

73. Carmichael, SL, Yang, W, Gilboa, S, Ailes, E, Correa, A, Botto, LD, Feldkamp, ML, Shaw, GM. Elevated body mass index and decreased diet quality among women and risk of birth defects in their offspring. Birth Defects Res A Clin Mol Teratol. 2016. 106:164-71. doi:10.1002/bdra.23471.

74. Castro, MB, Kac, G, Sichieri, R. Assessment of protein intake during pregnancy using a food frequency questionnaire and the effect on postpartum body weight variation. Cad Saude Publica. 2010. 26:2112-20.

75. Castro, MBT, Cunha, DB, Araujo, MC, Bezerra, IN, Adegboye, ARA, Kac, G, Sichieri, R. High protein diet promotes body weight loss among Brazilian postpartum women. Matern Child Nutr. 2018. :e12746. doi:10.1111/mcn.12746.

\section{Population}

Intervention/Exposure

Study Design

Outcome

Study Design; Outcome

Study Design; Outcome

Intervention/Exposure; Country

Population 
76. Castro, PS, de Castro, MBT, Kac, G. Adherence to dietary recommendations by the Institute of Medicine and the effect on body weight during pregnancy. Cadernos de Saude Publica. 2013. 29:1311-1321. doi:10.1590/S0102-311X2013000700006.

77. Centofanti, SF, Francisco, RPV, Phillippi, ST, Galletta, MAK, Sousa, AMS, Rodrigues, AS Curi, R, Brizot, ML. Maternal nutrient intake and fetal gastroschisis: A case-control study. Am J Med Genet A. 2019. doi:10.1002/ajmg.a.61265.

78. Chan, GM, McElligott, K, McNaught, T, Gill, G. Effects of dietary calcium intervention on adolescent mothers and newborns: a randomized controlled trial. Obstetrics and gynecology. 2006. 108:565-571. doi:10.1097/01.AOG.0000231721.42823.9e.

79. Chan, KK, Ho, LF, Lao, TT. Nutritional intake and placental size in gestational diabetic pregnancies--a preliminary observation. Placenta. 2003. 24:985-8.

80. Chandler-Laney, PC, Schneider, CR, Gower, BA, Granger, WM, Mancuso, MS, Biggio, JR. Association of late-night carbohydrate intake with glucose tolerance among pregnant African American women. Matern Child Nutr. 2016. 12:688-98. doi:10.1111/mcn.12181.

81. Chang, MW, Brown, R, Nitzke, S. Fast Food Intake in Relation to Employment Status, Stress, Depression, and Dietary Behaviors in Low-Income Overweight and Obese Pregnant Women. Matern Child Health J. 2016. 20:1506-17. doi:10.1007/s10995-0161949-5.

82. Chang, MW, Nitzke, S, Brown, R. Design and outcomes of a Mothers In Motion behavioral Intervention/Exposure; Population intervention pilot study. J Nutr Educ Behav. 2010. 42:S11-21. doi:10.1016/j.jneb.2010.01.010.

83. Chang, MW, Schaffir, J, Brown, R, Wegener, DT. Mediation by self-efficacy in the relation Intervention/Exposure between social support and dietary intake in low-income postpartum women who were overweight or obese. Appetite. 2019. 140:248-254. doi:10.1016/j.appet.2019.05.031.

84. Chang, MW, Tan, A, Schaffir, J. Relationships between stress, demographics and dietary intake behaviours among low-income pregnant women with overweight or obesity. Public Health Nutr. 2019. 22:1066-1074. doi:10.1017/s1368980018003385.

85. Charo, L, Lacoursiere, DY. Introduction: obesity and lifestyle issues in women. Clin Obstet Gynecol. 2014. 57:433-45. doi:10.1097/grf.0000000000000040.

86. Chatzi, Leda, Garcia, Raquel, Roumeliotaki, Theano, Basterrechea, Mikel, Begiristain, Haizea, Iñiguez, Carmen, Vioque, Jesus, Kogevinas, Manolis, Sunyer, Jordi. Mediterranean diet adherence during pregnancy and risk of wheeze and eczema in the first year of life: INMA (Spain) and RHEA (Greece) mother-child cohort studies. British Journal of Nutrition. 2013. 110:2058-2068. doi:10.1017/S0007114513001426.

87. Chen, GW, Ding, WH, Ku, HY, Chao, HR, Chen, HY, Huang, MC, Wang, SL. Alkylphenols in human milk and their relations to dietary habits in central Taiwan. Food Chem Toxicol. 2010. 48:1939-44. doi:10.1016/j.fct.2010.04.038. weight 
88. Chen, H, Wang, P, Han, Y, Ma, J, Troy, FA, 2nd, Wang, B. Evaluation of dietary intake of lactating women in China and its potential impact on the health of mothers and infants. BMC Womens Health. 2012. 12:18. doi:10.1186/1472-6874-12-18.

89. Chen, LW, Aris, IM, Bernard, JY, Tint, MT, Chia, A, Colega, M, Gluckman, PD, Shek, LP, Saw, SM, Chong, YS, Yap, F, Godfrey, KM, van Dam, RM, Chong, MF, Lee, YS. Associations of Maternal Dietary Patterns during Pregnancy with Offspring Adiposity from Birth Until 54 Months of Age. Nutrients. 2016. 9. doi:10.3390/nu9010002.

90. Chen, LW, Murrin, CM, Mehegan, J, Kelleher, CC, Phillips, CM. Maternal, but not paternal or grandparental, caffeine intake is associated with childhood obesity and adiposity: The Lifeways Cross-Generation Cohort Study. Am J Clin Nutr. 2019. 109:1648-1655. doi:10.1093/ajcn/nqz019.

91. Chen, LW, Navarro, P, Murrin, CM, Mehegan, J, Kelleher, CC, Phillips, CM. Maternal Dietary Glycemic and Insulinemic Indexes Are Not Associated with Birth Outcomes or Childhood Adiposity at 5 Years of Age in an Irish Cohort Study. J Nutr. 2019. 149:10371046. doi:10.1093/jn/nxz025.

92. Chen, LW, Tint, MT, Fortier, MV, Aris, IM, Bernard, JY, Colega, M, Gluckman, PD, Saw, SM, Chong, YS, Yap, F, Godfrey, KM, Kramer, MS, van Dam, RM, Chong, MFF, Lee, YS. Maternal macronutrient intake during pregnancy is associated with neonatal abdominal adiposity: The growing up in singapore towards healthy outcomes (GUSTO) study. Journal of Nutrition. 2016. 146:1571-1579. doi:10.3945/jn.116.230730.

93. Chen, Q, Feng, Y, Yang, H, Wu, W, Zhang, P, Wang, K, Wang, Y, Ko, J, Shen, J, Guo, L, Zhao, F, Du, W, Ru, S, Wang, S, Zhang, Y. A Vitamin Pattern Diet Is Associated with Decreased Risk of Gestational Diabetes Mellitus in Chinese Women: Results from a Case Control Study in Taiyuan, China. J Diabetes Res. 2019. 2019:5232308. doi:10.1155/2019/5232308.

94. Chen, X, Scholl, TO, Leskiw, M, Savaille, J, Stein, TP. Differences in maternal circulating fatty acid composition and dietary fat intake in women with gestational diabetes mellitus or mild gestational hyperglycemia. Diabetes Care. 2010. 33:2049-54. doi:10.2337/dc100693.

95. Cheng, YW, Chung, JH, Kurbisch-Block, I, Inturrisi, M, Shafer, S, Caughey, AB. Gestational weight gain and gestational diabetes mellitus: perinatal outcomes. Obstetrics \& Gynecology. 2008. 112:1015-1022.

96. Chia, AR, de Seymour, JV, Colega, M, Chen, LW, Chan, YH, Aris, IM, Tint, MT, Quah, PL, Godfrey, KM, Yap, F, Saw, SM, Baker, PN, Chong, YS, van Dam, RM, Lee, YS, Chong, MF. A vegetable, fruit, and white rice dietary pattern during pregnancy is associated with a lower risk of preterm birth and larger birth size in a multiethnic Asian cohort: the Growing Up in Singapore Towards healthy Outcomes (GUSTO) cohort study. Am J Clin Nutr. 2016. 104:1416-1423. doi:10.3945/ajcn.116.133892.

Study Design

\section{Intervention/Exposure}

\section{Outcome}

Study Design; Outcome

Study Design; Intervention/Exposure

Outcome; Comparator

Intervention/Exposure

Study Design 
97. Chia, AR, Tint, MT, Han, CY, Chen, LW, Colega, M, Aris, IM, Chua, MC, Tan, KH, Yap, F, Shek, LP, Chong, YS, Godfrey, KM, Fortier, MV, Lee, YS, Chong, MF. Adherence to a healthy eating index for pregnant women is associated with lower neonatal adiposity in a multiethnic Asian cohort: the Growing Up in Singapore Towards healthy Outcomes (GUSTO) Study. Am J Clin Nutr. 2018. 107:71-79. doi:10.1093/ajcn/nqx003.

98. Chiu, YH, Williams, PL, Gillman, MW, Hauser, R, Rifas-Shiman, SL, Bellavia, A, Fleisch, $A F$, Oken, $E$, Chavarro, JE. Maternal intake of pesticide residues from fruits and vegetables in relation to fetal growth. Environ Int. 2018. 119:421-428. doi:10.1016/j.envint.2018.07.014.

99. Chong, MF, Chia, AR, Colega, M, Tint, MT, Aris, IM, Chong, YS, Gluckman, P, Godfrey, KM, Kwek, K, Saw, SM, Yap, F, van Dam, RM, Lee, YS. Maternal Protein Intake during Pregnancy Is Not Associated with Offspring Birth Weight in a Multiethnic Asian Population. J Nutr. 2015. 145:1303-10. doi:10.3945/jn.114.205948.

100. Chortatos, A, Haugen, M, Iversen, PO, Vikanes, A, Magnus, P, Veierod, MB. Nausea and vomiting in pregnancy: associations with maternal gestational diet and lifestyle factors in the Norwegian Mother and Child Cohort Study. Bjog. 2013. 120:1642-53. doi:10.1111/1471-0528.12406.

101. Clerget-Froidevaux, MS, Sachs, LM. High-fat diet and pregnancy: Are you ready to take risks for your offspring? Endocrinology. 2017. 158:2716-2718. doi:10.1210/en.201700611.

102. Coathup, V, Northstone, K, Izadi, H, Wheeler, S, Smith, L. Do Maternal Dietary Antioxidants Modify the Relationship Between Binge Drinking and Small for Gestational Age? Findings from a Longitudinal Cohort Study. Alcoholism: Clinical and Experimental Research. 2018. 42:2196-2204. doi:10.1111/acer.13864.

103. Coelho Nde, L, Cunha, DB, Esteves, AP, Lacerda, EM, Theme Filha, MM. Dietary patterns in pregnancy and birth weight. Rev Saude Publica. 2015. 49:62. doi:10.1590/s0034-8910.2015049005403.

104. Cohen, TR, Koski, KG. Limiting excess weight gain in healthy pregnant women: importance of energy intakes, physical activity, and adherence to gestational weight gain guidelines. J Pregnancy. 2013. 2013:787032. doi:10.1155/2013/787032.

105. Cohen, TR, Plourde, H, Koski, KG. Are Canadian women achieving a fit pregnancy? A pilot study. Can J Public Health. 2010. 101:87-91.

106. Colatrella, A, Framarino, M, Toscano, V, Bongiovanni, M, Festa, C, Mattei, L, Merola, G, Bitterman, O, Maravalle, M, Napoli, A. Continuous glucose monitoring during breastfeeding in women with recent gestational diabetes mellitus. Diabetes Technol Ther 2012. 14:576-82. doi:10.1089/dia.2011.0266.

\section{Study Design}

\section{Outcome}


107. Colleran, HL, Lovelady, CA. Use of MyPyramid Menu Planner for Moms in a weight-loss intervention during lactation. J Acad Nutr Diet. 2012. 112:553-8. doi:10.1016/j.jand.2011.12.004.

108. Collins, CT, Chua, MC, Rajadurai, VS, McPhee, AJ, Miller, LN, Gibson, RA, Makrides, M. Higher protein and energy intake is associated with increased weight gain in pre-term infants. J Paediatr Child Health. 2010. 46:96-102. doi:10.1111/j.1440-1754.2009.01645.x.

109. Colon-Ramos, U, Racette, SB, Ganiban, J, Nguyen, TG, Kocak, M, Carroll, KN, Volgyi, E, Tylavsky, FA. Association between dietary patterns during pregnancy and birth size measures in a diverse population in Southern US. Nutrients. 2015. 7:1318-32. doi:10.3390/nu7021318.

110. Conway, MC, Mulhern, MS, McSorley, EM, van Wijngaarden, E, Strain, JJ, Myers, GJ, Davidson, PW, Shamlaye, CF, Yeates, AJ. Dietary determinants of polyunsaturated fatty acid (PUFA) status in a high fish-eating cohort during pregnancy. Nutrients. 2018. 10. doi:10.3390/nu10070927.

111. Cormick, G, Betran, AP, Harbron, J, Dannemann Purnat, T, Parker, C, Hall, D, Seuc, AH, Roberts, JM, Belizan, JM, Hofmeyr, GJ. Are women with history of pre-eclampsia starting a new pregnancy in good nutritional status in South Africa and Zimbabwe? BMC Pregnancy Childbirth. 2018. 18:236. doi:10.1186/s12884-018-1885-z.

112. Courtney, A, O'Brien, E, McAuliffe, F. The DASH (Dietary Approaches to Stop Hypertension) dietary pattern and blood pressure in pregnancy. BJOG. 2019. 126:42doi:10.1111/1471-0528.15633.

113. Courville, $A B$, Harel, $O$, Lammi-Keefe, $C J$. Consumption of a DHA-containing functional food during pregnancy is associated with lower infant ponderal index and cord plasma insulin concentration. Br J Nutr. 2011. 106:208-12. doi:10.1017/s0007114511000961.

114. Crume, TL, Brinton, JT, Shapiro, A, Kaar, J, Glueck, DH, Siega-Riz, AM, Dabelea, D. Maternal dietary intake during pregnancy and offspring body composition: The Healthy Start Study. Am J Obstet Gynecol. 2016. 215:609.e1-609.e8. doi:10.1016/j.ajog.2016.06.035

115. Cuco, G, Arija, V, Iranzo, R, Vila, J, Prieto, MT, Fernandez-Ballart, J. Association of maternal protein intake before conception and throughout pregnancy with birth weight. Acta Obstet Gynecol Scand. 2006. 85:413-21. doi:10.1080/00016340600572228.

116. Cuco, G, Fernandez-Ballart, J, Sala, J, Viladrich, C, Iranzo, R, Vila, J, Arija, V. Dietary patterns and associated lifestyles in preconception, pregnancy and postpartum. Eur $\mathrm{J}$ Clin Nutr. 2006. 60:364-71. doi:10.1038/sj.ejcn.1602324.

117. Cunha, MPL, Marques, RC, Dorea, JG. Influence of Maternal Fish Intake on the Anthropometric Indices of Children in the Western Amazon. Nutrients. 2018. 10. doi:10.3390/nu10091146.

Outcome

\section{Abstract}

\section{Outcome}

Intervention/Exposure; Outcome

Outcome

Outcome; Comparator

Intervention/Exposure; Outcome 
118. D. Association of food choices during pregnancy with gestational diabetes mellitus. Clinical Diabetology. 2017. 6:131-135. doi:10.5603/DK.2017.0022.

119. Dammann, KW, Smith, C. Factors affecting low-income women's food choices and the perceived impact of dietary intake and socioeconomic status on their health and weight. Journal of Nutrition Education \& Behavior. 2009. 41:242-253. doi:10.1016/j.jneb.2008.07.003.

120. Daundasekara, SS, Beasley, AD, O'Connor, DP, Sampson, M, Hernandez, D, Ledoux, T. Validation of the intuitive Eating Scale for pregnant women. Appetite. 2017. 112:201-209. doi:10.1016/j.appet.2017.02.001.

121. Davis, JN, Shearrer, GE, Tao, W, Hurston, SR, Gunderson, EP. Dietary variables associated with substantial postpartum weight retention at 1-year among women with GDM pregnancy. BMC Obes. 2017. 4:31. doi:10.1186/s40608-017-0166-0.

122. de Castro, MB, Freitas Vilela, AA, de Oliveira, AS, Cabral, M, de Souza, RA, Kac, G, Sichieri, R. Sociodemographic characteristics determine dietary pattern adherence during pregnancy. Public Health Nutr. 2016. 19:1245-51. doi:10.1017/s1368980015002700.

123. de Castro, MB, Kac, G, de Leon, AP, Sichieri, R. High-protein diet promotes a moderate postpartum weight loss in a prospective cohort of Brazilian women. Nutrition. 2009. 25:1120-8. doi:10.1016/j.nut.2009.02.006.

124. de Castro, MB, Sichieri, R, Barbosa Brito Fdos, S, Nascimento, S, Kac, G. Mixed dietary pattern is associated with a slower decline of body weight change during postpartum in a cohort of Brazilian women. Nutr Hosp. 2014. 29:519-25. doi:10.3305/nh.2014.29.3.7155.

125. De Jersey, SusanJ, Ross, LyndaJ, Himstedt, Kellie, Mclntyre, David H, Callaway, LeonieK. Weight gain and nutritional intake in obese pregnant women: Some clues for intervention. Nutrition \& Dietetics. 2011. 68:53-59. doi:10.1111/j.1747-0080.2010.01470.x.

126. de la Torre, NG, Assaf-Balut, C, Jimenez Varas, I, Del Valle, L, Duran, A, Fuentes, M, Del Prado, N, Bordiu, E, Valerio, JJ, Herraiz, MA, Izquierdo, N, Torrejon, MJ, Cuadrado, MA, de Miguel, P, Familiar, C, Runkle, I, Barabash, A, Rubio, MA, Calle-Pascual, AL. Effectiveness of Following Mediterranean Diet Recommendations in the Real World in the Incidence of Gestational Diabetes Mellitus (GDM) and Adverse Maternal-Foetal Outcomes: A Prospective, Universal, Interventional Study with a Single Group. The St Carlos Study. Nutrients. 2019. 11. doi:10.3390/nu11061210.

127. Deierlein, AL, Siega-Riz, AM, Herring, A. Dietary energy density but not glycemic load is associated with gestational weight gain. Am J Clin Nutr. 2008. 88:693-9. doi:10.1093/ajcn/88.3.693.

128. Del Prado, M, Villalpando, $\mathrm{S}$, Lance, A, Alfonso, E, Demmelmair, H, Koletzko, B. Contribution of dietary and newly formed arachidonic acid to milk secretion in women on low fat diets. Adv Exp Med Biol. 2000. 478:407-8. doi:10.1007/0-306-46830-1_50.
Study Design; Intervention/Exposure

Intervention/Exposure; Population

Intervention/Exposure

Intervention/Exposure

Study Design

Intervention/Exposure; Population

Population; Country

Comparator

Study Design; Intervention/Exposure

Intervention/Exposure

Book chapter 
129. Della Libera, $B$, Ribeiro Baiao, $M$, de Souza Santos, MM, Padilha, $P$, Dutra Alves, $P$, Saunders, C. Adherence of pregnant women to dietary counseling and adequacy of total gestational weight gain. Nutr Hosp. 2011. 26:79-85.

130. Denguezli, W, Faleh, R, Fessi, A, Yassine, A, Hajjaji, A, Laajili, H, Sakouhi, M. Risk factors of fetal macrosomia: role of maternal nutrition. Tunis Med. 2009. 87:564-8.

131. Deniz Ç, D, Özler, S, Sayın, FK, Eryılmaz, MA. Associations between night eating syndrome and metabolic parameters in pregnant women. Turk Jinekoloji ve Obstetrik Dernegi Dergisi. 2019. 16:107-111. doi:10.4274/tjod.galenos.2019.77864.

132. Derbyshire, E, Davies, J, Costarelli, V, Dettmar, P. Prepregnancy body mass index and dietary intake in the first trimester of pregnancy. J Hum Nutr Diet. 2006. 19:267-73. doi:10.1111/j.1365-277X.2006.00705.x.

133. deRosset, L, Berry, DC, Sanchez-Lugo, L, Ritter, K, Purdum, C, Santolim, V, Gilliland, R, Pender, L. Mama Sana .. Usted Sana: Lessons Learned From a Postpartum Weight Loss Intervention for Hispanic Women With Infants Six Months or Less. Hispanic Health Care International. 2013. 11:78-86. doi:10.1891/1540-4153.11.2.78.

134. DeSantiago, S, Alonso, L, Ramírez, I, Ortíz, N, Tovar, AR, Torres, N, Bourges, H. Metabolizable energy from a predominantly vegetable diet consumed by Mexican rural lactating women. Nutrition Research. 2000. 20:215-224. doi:10.1016/S0271. 5317(99)00154-2.

135. Dhana, K, Haines, J, Liu, G, Zhang, C, Wang, X, Field, AE, Chavarro, JE, Sun, Q. Association between maternal adherence to healthy lifestyle practices and risk of obesity in offspring: results from two prospective cohort studies of mother-child pairs in the United States. Bmj. 2018. 362:k2486. doi:10.1136/bmj.k2486.

136. Dharod, JM, Croom, JE, Sady, CG. Food Insecurity: Its Relationship to Dietary Intake and Body Weight among Somali Refugee Women in the United States. Journal of Nutrition Education \& Behavior. 2013. 45:47-53. doi:10.1016/j.jneb.2012.03.006.

137. Di Carlo, C, Iannotti, G, Sparice, S, Chiacchio, MP, Greco, E, Tommaselli, GA, Nappi, C. Intervention/Exposure; Comparator The role of a personalized dietary intervention in managing gestational weight gain: a prospective, controlled study in a low-risk antenatal population. Archives of gynecology and obstetrics. 2014. 289:765-770. doi:10.1007/s00404-013-3054-y.

138. Diemert, A, Lezius, S, Pagenkemper, M, Hansen, G, Drozdowska, A, Hecher, K, Arck, $P$, Zyriax, BC. Maternal nutrition, inadequate gestational weight gain and birth weight: results from a prospective birth cohort. BMC Pregnancy Childbirth. 2016. 16:224. doi:10.1186/s12884-016-1012-y.

139. Dieting okay for breastfeeding mothers. J Natl Med Assoc. 2000. 92:A14. 
140. Dikensoy, E, Balat, O, Cebesoy, B, Ozkur, A, Cicek, H, Can, G. Effect of fasting during Ramadan on fetal development and maternal health. J Obstet Gynaecol Res. 2008. 34:494-8. doi:10.1111/j.1447-0756.2008.00814.x.

141. Dodd, JM, Cramp, C, Sui, Z, Yelland, LN, Deussen, AR, Grivell, RM, Moran, LJ, Crowther, CA, Turnbull, D, McPhee, AJ, Wittert, G, Owens, JA, Robinson, JS. The effects of antenatal dietary and lifestyle advice for women who are overweight or obese on maternal diet and physical activity: the LIMIT randomised trial. BMC Med. 2014. 12:161. doi:10.1186/s12916-014-0161-y.

142. Dodd, JM, Deussen, AR, Mohamad, I, Rifas-Shiman, SL, Yelland, LN, Louise, J, McPhee, AJ, Grivell, RM, Owens, JA, Gillman, MW, Robinson, JS. The effect of antenatal lifestyle advice for women who are overweight or obese on secondary measures of neonatal body composition: the LIMIT randomised trial. Bjog. 2016. 123:244-53. doi:10.1111/14710528.13796.

143. Dodd, JM, Grivell, RM, Owens, JA. Antenatal Dietary and Lifestyle Interventions for Women Who are Overweight or Obese: outcomes from the LIMIT Randomized Trial. Current nutrition reports. 2014. 3:392-399. doi:10.1007/s13668-014-0101-7.

144. Dodd, JM, Kannieappan, LM, Grivell, RM, Deussen, AR, Moran, LJ, Yelland, LN, Owens, JA. Effects of an antenatal dietary intervention on maternal anthropometric measures in pregnant women with obesity. Obesity (Silver Spring). 2015. 23:1555-62. doi:10.1002/oby.21145.

145. Dodd, JM, Newman, A, Moran, LJ, Deussen, AR, Grivell, RM, Yelland, LN, Crowther, CA, McPhee, AJ, Wittert, G, Owens, JA, Turnbull, D, Robinson, JS. The effect of antenatal dietary and lifestyle advice for women who are overweight or obese on emotional wellbeing: the LIMIT randomized trial. Acta Obstet Gynecol Scand. 2016. 95:309-18. doi:10.1111/aogs.12832.

146. Dodd, JM. Dietary and lifestyle advice for pregnant women who are overweight or obese: the LIMIT randomized trial. Annals of nutrition \& metabolism. 2014. 64:197-202. doi:10.1159/000365018.

147. Dominguez, LJ, Martinez-Gonzalez, MA, Basterra-Gortari, FJ, Gea, A, Barbagallo, M, BesRastrollo, M. Fast food consumption and gestational diabetes incidence in the SUN project. PLoS One. 2014. 9:e106627. doi:10.1371/journal.pone.0106627.

148. Donazar-Ezcurra, M, Lopez-Del Burgo, C, Martinez-Gonzalez, MA, Basterra-Gortari, FJ, de Irala, J, Bes-Rastrollo, M. Pre-pregnancy adherences to empirically derived dietary patterns and gestational diabetes risk in a Mediterranean cohort: the Seguimiento Universidad de Navarra (SUN) project. Br J Nutr. 2017. 118:715-721. doi:10.1017/s0007114517002537. 
149. Donazar-Ezcurra, M, Lopez-Del Burgo, C, Martinez-Gonzalez, MA, Basterra-Gortari, FJ, de Irala, J, Bes-Rastrollo, M. Soft drink consumption and gestational diabetes risk in the SUN project. Clin Nutr. 2018. 37:638-645. doi:10.1016/j.clnu.2017.02.005.

150. Donnelly, JM, Walsh, JM, Byrne, J, Molloy, EJ, McAuliffe, FM. Impact of maternal diet on neonatal anthropometry: a randomized controlled trial. Pediatr Obes. 2015. 10:52-6. doi:10.1111/j.2047-6310.2013.00216.x.

151. Dos Santos, $K$, Tavares Patricio, $P$, Santana Vieira Lima, T, Cavalcante de Barros, $D$ Saunders, C. A pilot intervention to reduce postpartum weight retention at primary health care in Brazil. Nutr Hosp. 2019. doi:10.20960/nh.02508.

152. Drehmer, M, Camey, SA, Nunes, MA, Duncan, BB, Lacerda, M, Pinheiro, AP, Schmidt, MI. Fibre intake and evolution of BMl: from pre-pregnancy to postpartum. Public Health Nutr. 2013. 16:1403-13. doi:10.1017/s1368980012003849.

153. Drouillet, $P$, Forhan, $A$, De Lauzon-Guillain, $B$, Thiébaugeorges, $O$, Goua, $V$, Magnin, G, Schweitzer, $M$, Kaminski, $M$, Ducimetière, $P$, Charles, $M$. Maternal fatty acid intake and fetal growth: evidence for an association in overweight women. The 'EDEN mother-child' cohort (study of pre- and early postnatal determinants of the child's development and health). British Journal of Nutrition. 2009. 101:583-591. doi:10.1017/S0007114508025038.

154. Drouillet, $P$, Kaminski, $M$, De Lauzon-Guillain, $B$, Forhan, $A$, Ducimetiere, $P$, Schweitzer, M, Magnin, G, Goua, V, Thiebaugeorges, O, Charles, MA. Association between maternal seafood consumption before pregnancy and fetal growth: evidence for an association in overweight women. The EDEN mother-child cohort. Paediatr Perinat Epidemiol. 2009. 23:76-86. doi:10.1111/j.1365-3016.2008.00982.x.

155. Dubois, L, Diasparra, M, Bédard, B, Colapinto, CK, Fontaine-Bisson, B, Tremblay, RE, Fraser, WD. Adequacy of nutritional intake during pregnancy in relation to prepregnancy BMI: results from the 3D Cohort Study. British Journal of Nutrition. 2018. 120:335-344. doi:10.1017/S0007114518001393.

156. Dujmovic, M, Kresic, G, Mandic, ML, Kenjeric, D, Cvijanovic, O. Changes in dietary intake and body weight in lactating and non-lactating women: prospective study in northern coastal Croatia. Coll Antropol. 2014. 38:179-87.

157. Durham, HA, Lovelady, CA, Brouwer, RJ, Krause, KM, Ostbye, T. Comparison of dietary intake of overweight postpartum mothers practicing breastfeeding or formula feeding. J Am Diet Assoc. 2011. 111:67-74. doi:10.1016/j.jada.2010.10.001.

158. Easter, A, Naumann, U, Northstone, K, Schmidt, U, Treasure, J, Micali, N. A longitudinal investigation of nutrition and dietary patterns in children of mothers with eating disorders. $J$ Pediatr. 2013. 163:173-8.e1. doi:10.1016/j.jpeds.2012.11.092. 
159. Ebrahimi, F, Shariff, ZM, Tabatabaei, SZ, Fathollahi, MS, Mun, CY, Nazari, M. Relationship between sociodemographics, dietary intake, and physical activity with gestational weight gain among pregnant women in Rafsanjan City, Iran. J Health Popul Nutr. 2015. 33:168-76.

160. Eichler, J, Schmidt, R, Hiemisch, A, Kiess, W, Hilbert, A. Gestational weight gain, physical activity, sleep problems, substance use, and food intake as proximal risk factors of stress and depressive symptoms during pregnancy. BMC Pregnancy Childbirth. 2019. 19:175. doi:10.1186/s12884-019-2328-1.

161. Ellery, THP, Sampaio, HAC, Carioca, AAF, Silva, Bydc, Alves, JAG, Da Silva Costa, F, Araujo Junior, E, Melo, MLP. Association between Dietary Glycemic Index and Excess Weight in Pregnant Women in the First Trimester of Pregnancy. Rev Bras Ginecol Obstet. 2019. 41:4-10. doi:10.1055/s-0038-1676096.

162. Elliott, SA, Pereira, LCR, McCargar, LJ, Prado, CM, Bell, RC. Trajectory and determinants of change in lean soft tissue over the postpartum period. Br J Nutr. 2019. 121:1137-1145. doi:10.1017/s0007114518002015.

163. Elvebakk, T, Mostad, IL, Morkved, S, Salvesen, KA, Stafne, SN. Dietary Intakes and Dietary Quality during Pregnancy in Women with and without Gestational Diabetes Mellitus-A Norwegian Longitudinal Study. Nutrients. 2018. 10. doi:10.3390/nu10111811.

164. Englund-Ogge, L, Brantsaeter, AL, Juodakis, J, Haugen, M, Meltzer, HM, Jacobsson, B, Sengpiel, V. Associations between maternal dietary patterns and infant birth weight, small and large for gestational age in the Norwegian Mother and Child Cohort Study. Eur J Clin Nutr. 2018. doi:10.1038/s41430-018-0356-y.

165. Eshriqui, I, Vilela, AA, Rebelo, F, Farias, DR, Castro, MB, Kac, G. Gestational dietary patterns are not associated with blood pressure changes during pregnancy and early postpartum in a Brazilian prospective cohort. Eur J Nutr. 2016. 55:21-32. doi:10.1007/s00394-014-0819-4.

166. Estimated dietary vitamin $D$ intake during pregnancy. Proceedings of the nutrition society. 2017. Conference: Nutrition Society Irish Section Conference 2017: What Governs What We Eat? United Kingdom. 76:E62. doi:10.1017/S0029665117001355.

167. Facchinetti, F, Gambigliani Zoccoli, S, Petrella, E, Bertarini, V, Di Cerbo, L, Neri, I. 160: counseling lifestyle changes in overweight/obese pregnant women: a randomized controlled trial. American journal of obstetrics and gynecology. 2019. 220:S120-S121. doi:10.1016/j.ajog.2018.11.181.

168. Facchinetti, F, Vijay, V, Petrella, E, Gambigliani Zoccoli, S, Bertarini, V, Di Cerbo, L, Neri, I. 78: impact of glycemic-index(Gl) reduction on birthweight in overweight/obese pregnant women enrolled in a lifestyle program. American journal of obstetrics and gynecology. 2019. 220:S62-. doi:10.1016/j.ajog.2018.11.086.

Study Design; Intervention/Exposure

Intervention/Exposure

Study Design

Intervention/Exposure; Population

Intervention/Exposure; Outcome

Outcome

Outcome

Abstract

Abstract

Abstract 
169. Fadakar, SK, Ghavi, A, Niknami, M, Kazemnejad, LE. Relationship between mothers' gt $<$ GT $>$ nutritional status and weight gain during pregnancy with low birth weight. Journal of Guilan University of Medical Sciences. 2012. 21:27-35.

170. Fadzil, F, Shamsuddin, K, Sharifa, EWP, Tamil, AM, Ahmad, S, Hayi, NSA, Samad, AA, Ismail, R, Shauki, NIA. Predictors of postpartum weight retention among urban Malaysian mothers: A prospective cohort study. Obesity Research \& Clinical Practice. 2018. 12:493499. doi:10.1016/j.orcp.2018.06.003.

171. Fahey, CA, Chevrier, J, Crause, M, Obida, M, Bornman, R, Eskenazi, B. Seasonality of antenatal care attendance, maternal dietary intake, and fetal growth in the VHEMBE birth cohort, South Africa. PLoS ONE. 2019. 14. doi:10.1371/journal.pone.0222888.

172. Fahey, MC, Wayne Talcott, G, Cox Bauer, CM, Bursac, Z, Gladney, L, Hare, ME, Harvey, J, Little, M, McCullough, D, Hryshko-Mullen, AS, Klesges, RC, Kocak, M, Waters, TM, Krukowski, RA. Moms fit 2 fight: Rationale, design, and analysis plan of a behavioral weight management intervention for pregnant and postpartum women in the U.S. military. Contemporary Clinical Trials. 2018. 74:46-54. doi:10.1016/j.cct.2018.09.012.

173. Fereidooni, B, Jenabi, E. The use of omega 3 on pregnancy outcomes: a single-center study. J Pak Med Assoc. 2014. 64:1363-5.

174. Ferland, S, O'Brien, HT. Maternal dietary intake and pregnancy outcome. J Reprod Med. 2003. 48:86-94.

175. Ferranti, EP, Hartman, TJ, Elliott, AJ, Mitchell, DC, Angal, J, Nickleach, D, Bellissimo, M, Breslow, R. Diet Quality of Pregnant American Indian Women in the Northern Plains. Prev Chronic Dis. 2019. 16:E53. doi:10.5888/pcd16.180536.

176. Ferrara, P, Sandullo, F, Di Ruscio, F, Franceschini, G, Peronti, B, Blasi, V, Bietolini, S, Ruggiero, A. The impact of lacto-ovo-/lacto-vegetarian and vegan diets during pregnancy on the birth anthropometric parameters of the newborn. J Matern Fetal Neonatal Med. 2019. :1-7. doi:10.1080/14767058.2019.1590330.

177. Fiese, BH, Musaad, S, Bost, KK, McBride, BA, Lee, SY, Teran-Garcia, M, Donovan, SM. The STRONG Kids 2 Birth Cohort Study: A Cell-to-Society Approach to Dietary Habits and Weight Trajectories across the First 5 Years of Life. Curr Dev Nutr. 2019. 3:nzz007. doi:10.1093/cdn/nzz007.

178. Florencio, TM, Bueno, NB, Clemente, AP, Albuquerque, FC, Britto, RP, Ferriolli, E, Sawaya, AL. Weight gain and reduced energy expenditure in low-income Brazilian women living in slums: a 4-year follow-up study. Br J Nutr. 2015. 114:462-71. doi:10.1017/s0007114515001816.

Intervention/Exposure

\section{Outcome; Country}

Intervention/Exposure; Outcome

Intervention/Exposure; Outcome

Outcome

\section{Outcome}

Intervention/Exposure; Outcome

Intervention/Exposure; Population

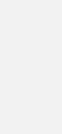


179. Flynn, A, Poston, L, Goff, L. The UK pregnancies better eating and activity trial (UPBEAT) intervention in women with obesity; nutritional responses according to ethnic and BMI subgroups. BJOG: an international journal of obstetrics and gynaecology. Conference: 19th annual conference of the british maternal and fetal medicine society, BMFMS 2017. Netherlands. 2017. 124:98. doi:10.1111/1471-0528.14588.

180. Flynn, AC, Schneeberger, C, Seed, PT, Barr, S, Poston, L, Goff, LM. The Effects of the UK Pregnancies Better Eating and Activity Trial Intervention on Dietary Patterns in Obese Pregnant Women Participating in a Pilot Randomized Controlled Trial. Nutr Metab Insights. 2015. 8:79-86. doi:10.4137/nmi.S29529.

181. Flynn, AC, Seed, PT, Patel, N, Barr, S, Bell, R, Briley, AL, Godfrey, KM, Nelson, SM, Oteng-Ntim, E, Robinson, SM, Sanders, TA, Sattar, N, Wardle, J, Poston, L, Goff, LM. Dietary patterns in obese pregnant women; influence of a behavioral intervention of diet and physical activity in the UPBEAT randomized controlled trial. Int J Behav Nutr Phys Act. 2016. 13:124. doi:10.1186/s12966-016-0450-2.

182. Forbes, LE, Graham, JE, Berglund, C, Bell, RC. Dietary change during pregnancy and women's reasons for change. Nutrients. 2018. 10. doi:10.3390/nu10081032.

183. Fowler, JK, Evers, SE, Campbell, MK. Inadequate dietary intakes among pregnant women. Can J Diet Pract Res. 2012. 73:72-7. doi:10.3148/73.2.2012.72.

184. Fowles, ER, Gabrielson, M. First trimester predictors of diet and birth outcomes in lowincome pregnant women. J Community Health Nurs. 2005. 22:117-30. doi:10.1207/s15327655jchn2202_5.

185. Fowles, ER, Timmerman, GM, Bryant, M, Kim, S. Eating at fast-food restaurants and dietary quality in low-income pregnant women. West J Nurs Res. 2011. 33:630-51. doi:10.1177/0193945910389083.

186. Fowles, ER, Walker, LO. Correlates of dietary quality and weight retention in postpartum women. J Community Health Nurs. 2006. 23:183-97. doi:10.1207/s15327655jchn2303_5.

187. Fukuda, Y, Yamamoto, S, Nishida, K, Takaoka, Y, Kameda, M. A study on the effect of nutrient intake on the body mass index of mothers of children with food allergies. Asian Pac J Allergy Immunol. 2019. doi:10.12932/ap-260718-0377.

188. Gallagher, D, Rosenn, B, Toro-Ramos, T, Paley, C, Gidwani, S, Horowitz, M, Crane, J, Lin, S, Thornton, JC, Pi-Sunyer, X, Toro-Ramos, T, Pi-Sunyer, X. Greater Neonatal FatFree Mass and Similar Fat Mass Following a Randomized Trial to Control Excess Gestational Weight Gain. Obesity (19307381). 2018. 26:578-587. doi:10.1002/oby.22079.

189. Garay, SM, Savory, KA, Sumption, L, Penketh, R, Janssen, AB, John, RM. The Grown in Wales Study: Examining dietary patterns, custom birthweight centiles and the risk of delivering a small-for-gestational age (SGA) infant. PLoS One. 2019. 14:e0213412. doi:10.1371/journal.pone.0213412.

\section{Outcome}

\section{Outcome}

Population

Population; Outcome

Intervention/Exposure; Outcome

\section{Study Design}


190. Garg, A, Kashyap, S. Effect of counseling on nutritional status during pregnancy. Indian J Pediatr. 2006. 73:687-92.

191. Gazquez, A, Uhl, O, Ruiz-Palacios, M, Gill, C, Patel, N, Koletzko, B, Poston, L, Larque, E. Placental lipid droplet composition: Effect of a lifestyle intervention (UPBEAT) in obese pregnant women. Biochim Biophys Acta Mol Cell Biol Lipids. 2018. 1863:998-1005. doi:10.1016/j.bbalip.2018.04.020.

192. Gennaro, S, Fehder, W. Health behaviors in postpartum women. Family \& Community Health. 2000. 22:16-26.

193. George, GC, Hanss-Nuss, H, Milani, TJ, Freeland-Graves, JH. Food choices of lowincome women during pregnancy and postpartum. J Am Diet Assoc. 2005. 105:899-907. doi:10.1016/j.jada.2005.03.028.

194. George, GC, Milani, TJ, Hanss-Nuss, H, Freeland-Graves, JH. Compliance with dietary guidelines and relationship to psychosocial factors in low-income women in late postpartum. J Am Diet Assoc. 2005. 105:916-26. doi:10.1016/j.jada.2005.03.009.

195. Geraghty, AA, O'Brien, EC, Alberdi, G, Horan, MK, Donnelly, J, Larkin, E, Segurado, R, Mehegan, J, Molloy, EJ, McAuliffe, FM. Maternal protein intake during pregnancy is associated with child growth up to 5 years of age, but not through insulin-like growth factor-1: findings from the ROLO study. Br J Nutr. 2018. 120:1252-1261. doi:10.1017/s0007114518002611.

196. Gesteiro, E, Bastida, S, Sanchez Muniz, FJ. Effects of maternal glucose tolerance, pregnancy diet quality and neonatal insulinemia upon insulin resistance/sensitivity biomarkers in normoweight neonates. Nutr Hosp. 2011. 26:1447-55. doi:10.1590/s021216112011000600036.

197. Ghani, RA, Shyam, S, Arshad, F, Wahab, NA, Chinna, K, Safii, NS, Nisak, MY, Kamaruddin, NA. The influence of fasting insulin level in post-gestational diabetes mellitus women receiving low-glycaemic-index diets. Nutr Diabetes. 2014. 4:e107. doi:10.1038/nutd.2014.5.

198. Gicevic, S, Gaskins, AJ, Fung, TT, Rosner, B, Tobias, DK, Isanaka, S, Willett, WC Evaluating pre-pregnancy dietary diversity vs. dietary quality scores as predictors of gestational diabetes and hypertensive disorders of pregnancy. PLoS ONE. 2018. 13. doi:10.1371/journal.pone.0195103.

199. Giesta, JM, Zoche, E, Correa, RDS, Bosa, VL. Associated factors with early introduction of ultra-processed foods in feeding of children under two years old. Cien Saude Colet. 2019. 24:2387-2397. doi:10.1590/1413-81232018247.24162017.

\section{Outcome}

\section{Outcome}

\section{Study Design}


200. Gignac, F, Romaguera, D, Fernandez-Barres, S, Phillipat, C, Garcia Esteban, R, LopezVicente, M, Vioque, J, Fernandez-Somoano, A, Tardon, A, Iniguez, C, Lopez-Espinosa, MJ, Garcia de la Hera, M, Amiano, P, Ibarluzea, J, Guxens, M, Sunyer, J, Julvez, J. Maternal nut intake in pregnancy and child neuropsychological development up to 8 years old: a population-based cohort study in Spain. Eur J Epidemiol. 2019. 34:661-673. doi:10.1007/s10654-019-00521-6.

201. Gingras, V, Paradis, AM, Tchernof, A, Weisnagel, SJ, Robitaille, J. Relationship between the adoption of preventive practices and the metabolic profile of women with prior gestational diabetes mellitus. Appl Physiol Nutr Metab. 2012. 37:1232-8. doi:10.1139/h2012-114.

202. Giroux, I, Inglis, SD, Lander, S, Gerrie, S, Mottola, MF. Dietary intake, weight gain, and birth outcomes of physically active pregnant women: a pilot study. Appl Physiol Nutr Metab. 2006. 31:483-9. doi:10.1139/h06-024.

203. Goletzke, J, Buyken, AE, Louie, JC, Moses, RG, Brand-Miller, JC. Dietary micronutrient intake during pregnancy is a function of carbohydrate quality. Am J Clin Nutr. 2015. 102:626-32. doi:10.3945/ajcn.114.104836.

204. Gonzalez, HF, Malpeli, A, Mansur, JL, De Santiago, S, Etchegoyen, GS. Changes in body composition in lactating adolescent mothers. Arch Latinoam Nutr. 2005. 55:252-6.

205. Gonzalez-Clemente, JM, Carro, $O$, Gallach, I, Vioque, J, Humanes, A, Sauret, C, Abella, M, Gimenez-Perez, G, Mauricio, D. Increased cholesterol intake in women with gestational diabetes mellitus. Diabetes Metab. 2007. 33:25-9. doi:10.1016/j.diabet.2006.07.002.

206. Gonzalez-Nahm, S, Mendez, M, Robinson, W, Murphy, SK, Hoyo, C, Hogan, V, Rowley, D. Low maternal adherence to a Mediterranean diet is associated with increase in methylation at the MEG3-IG differentially methylated region in female infants. Environ Epigenet. 2017. 3:dvx007. doi:10.1093/eep/dvx007.

207. Goodarzi-Khoigani, M, Mahmoodabad, SSM, Moghadam, MHB, Nadjarzadeh, A, Mardanian, F, Fallahzadeh, $\mathrm{H}$, Dadkhah-Tirani, A. Prevention of insulin resistance by dietary intervention among pregnant mothers: A randomized controlled trial. International Journal of Preventive Medicine. 2017. 8. doi:10.4103/ijpvm.IJPVM_405_16.

208. Goodman, M, Thomson, J, Landry, A. Nutrition Environment Scores of Local Food Retailers and Proximity to Study Participants' Residence in Rural South (P04-157-19). Curr Dev Nutr. 2019. 3. doi:10.1093/cdn/nzz051.P04-157-19.

209. Grandy, M, Snowden, JM, Boone-Heinonen, J, Purnell, JQ, Thornburg, KL, Marshall, NE. Poorer maternal diet quality and increased birth weight(). J Matern Fetal Neonatal Med. 2018. 31:1613-1619. doi:10.1080/14767058.2017.1322949.
Intervention/Exposure

Population; Outcome

Intervention/Exposure

Intervention/Exposure; Outcome

Intervention/Exposure

Intervention/Exposure; Outcome

Population

Intervention/Exposure

Abstract

Study Design 
210. Gray-Donald, K, Robinson, E, Collier, A, David, K, Renaud, L, Rodrigues, S. Intervening to reduce weight gain in pregnancy and gestational diabetes mellitus in Cree communities: an evaluation. Cmaj. 2000. 163:1247-51.

211. Gresham, E, Collins, CE, Mishra, GD, Byles, JE, Hure, AJ. Diet quality before or during Outcome pregnancy and the relationship with pregnancy and birth outcomes: the Australian Longitudinal Study on Women's Health. Public health nutrition. 2016. 19:2975-2983.

212. Guelinckx, I, Devlieger, R, Mullie, $P$, Vansant, G. Effect of lifestyle intervention on dietary habits, physical activity, and gestational weight gain in obese pregnant women: a randomized controlled trial. Am J Clin Nutr. 2010. 91:373-80. doi:10.3945/ajcn.2009.28166.

213. Guilloty, NI, Soto, R, Anzalota, L, Rosario, Z, Cordero, JF, Palacios, C. Diet, Prepregnancy BMI, and Gestational Weight Gain in Puerto Rican Women. Matern Child Health J. 2015. 19:2453-61. doi:10.1007/s10995-015-1764-4.

214. Gunther, J, Hoffmann, J, Kunath, J, Spies, M, Meyer, D, Stecher, L, Rosenfeld, E, Kick, L, Rauh, K, Hauner, H. Effects of a Lifestyle Intervention in Routine Care on Prenatal Dietary Behavior-Findings from the Cluster-Randomized GeliS Trial. J Clin Med. 2019. 8. doi:10.3390/jcm8070960.

215. Gunther, J, Hoffmann, J, Rauh, K, Kunath, J, Rosenfeld, E, Kick, L, Stecher, L, Hauner, H. Abstract The effect of lifestyle counselling on dietary behavior of pregnant women - Secondary results of the randomised controlled GeliS trial. Obesity facts. 2019. 12:171-. doi:10.1159/000489691.

216. Gunther, J, Hoffmann, J, Spies, M, Meyer, D, Kunath, J, Stecher, L, Rosenfeld, E, Kick, L, Rauh, K, Hauner, H. Associations between the Prenatal Diet and Neonatal Outcomes-A Secondary Analysis of the Cluster-Randomised GeliS Trial. Nutrients. 2019. 11. doi:10.3390/nu11081889.

217. Gur, EB, Turan, GA, Ince, O, Karadeniz, M, Tatar, S, Kasap, E, Sahin, N, Guclu, S. Effect of Ramadan fasting on metabolic markers, dietary intake and abdominal fat distribution in pregnancy. Hippokratia. 2015. 19:298-303.

218. Guzel, Al, Cinar, M, Erkilinc, S, Aksoy, RT, Yumusak, OH, Celik, F, Celik, Y. Association between adverse perinatal outcomes and amino acid levels measured with nutrient questionnaire in adolescent pregnancies. J Chin Med Assoc. 2016. 79:335-9. doi:10.1016/j.jcma.2015.12.008.

219. Haakstad, LAH, Voldner, N, Bø, K. Pregnancy and advanced maternal age-The associations between regular exercise and maternal and newborn health variables. Acta Obstetricia et Gynecologica Scandinavica. 2019. doi:10.1111/aogs.13738. 
220. Hagberg, LA, Brekke, HK, Bertz, F, Winkvist, A. Cost-utility analysis of a randomized controlled weight loss trial among lactating overweight/obese women. BMC Public Health. 2014. 14:38. doi:10.1186/1471-2458-14-38.

221. Haire-Joshu, DL, Schwarz, CD, Peskoe, SB, Budd, EL, Brownson, RC, Joshu, CE. A Intervention/Exposure; Population group randomized controlled trial integrating obesity prevention and control for postpartum adolescents in a home visiting program. Int J Behav Nutr Phys Act. 2015. 12:88. doi:10.1186/s12966-015-0247-8.

222. Hajianfar, H, Esmaillzadeh, A, Feizi, A, Shahshahan, Z, Azadbakht, L. Major Maternal Dietary Patterns during Early Pregnancy and Their Association with Neonatal Anthropometric Measurement. Biomed Res Int. 2018. 2018:4692193. doi:10.1155/2018/4692193.

223. Hajianfar, H, Esmaillzadeh, A, Feizi, A, Shahshahan, Z, Azadbakht, L. The association between major dietary patterns and pregnancy-related complications. Archives of Iranian Medicine. 2018. 21:443-451.

224. Halldorsson, TI, Meltzer, HM, Thorsdottir, I, Knudsen, V, Olsen, SF. Is high consumption of fatty fish during pregnancy a risk factor for fetal growth retardation? A study of 44,824 Danish pregnant women. Am J Epidemiol. 2007. 166:687-96. doi:10.1093/aje/kwm133.

225. Halldorsson, TI, Thorsdottir, I, Meltzer, HM, Strom, M, Olsen, SF. Dioxin-like activity in plasma among Danish pregnant women: dietary predictors, birth weight and infant development. Environ Res. 2009. 109:22-8. doi:10.1016/j.envres.2008.08.011.

226. Hamad, R, Collin, DF, Baer, RJ, Jelliffe-Pawlowski, LL. Association of Revised WIC Food Intervention/Exposure Package With Perinatal and Birth Outcomes: A Quasi-Experimental Study. JAMA Pediatrics. 2019. 173:845-852. doi:10.1001/jamapediatrics.2019.1706.

227. Han, SY, Brewis, AA. Influence of weight concerns on breastfeeding: Evidence from the Norwegian mother and child cohort study. Am J Hum Biol. 2018. 30:23-28. doi:10.1002/ajhb.23086.

228. Harley, K, Eskenazi, B, Block, G. The association of time in the US and diet during pregnancy in low-income women of Mexican descent. Paediatr Perinat Epidemiol. 2005. 19:125-34. doi:10.1111/j.1365-3016.2005.00640.x.

229. Harmon, KA, Gerard, L, Jensen, DR, Kealey, EH, Hernandez, TL, Reece, MS, Barbour, LA, Bessesen, DH. Continuous glucose profiles in obese and normal-weight pregnant women on a controlled diet: metabolic determinants of fetal growth. Diabetes Care. 2011. 34:2198-204. doi:10.2337/dc11-0723.

\section{Outcome}

\section{Outcome}

Outcome

Intervention/Exposure; Outcome

Comparator; Association btw DP and GWG not analyzed

\section{Intervention/Exposure}


230. Harreiter, J, Simmons, D, Desoye, G, Corcoy, R, Adelantado, JM, Devlieger, R, Galjaard, S, Damm, P, Mathiesen, ER, Jensen, DM, Andersen, LLT, Dunne, F, Lapolla, A, Dalfra, MG, Bertolotto, A, Wender-Ozegowska, E, Zawiejska, A, Mantaj, U, Hill, D, Jelsma, JGM, Snoek, FJ, Leutner, M, Lackinger, C, Worda, C, Bancher-Todesca, D, Scharnagl, H, van Poppel, MNM, Kautzky-Willer, A. Nutritional Lifestyle Intervention in Obese Pregnant Women, Including Lower Carbohydrate Intake, Is Associated With Increased Maternal Free Fatty Acids, 3-beta-Hydroxybutyrate, and Fasting Glucose Concentrations: A Secondary Factorial Analysis of the European Multicenter, Randomized Controlled DALI Lifestyle Intervention Trial. Diabetes Care. 2019. doi:10.2337/dc19-0418.

231. Haruna, M, Shiraishi, M, Matsuzaki, M, Yatsuki, Y, Yeo, S. Effect of tailored dietary guidance for pregnant women on nutritional status: A double-cohort study. Matern Child Nutr. 2017. 13. doi:10.1111/mcn.12391.

232. Hassiotou, F, Geddes, DT. Programming of appetite control during breastfeeding as a preventative strategy against the obesity epidemic. J Hum Lact. 2014. 30:136-42. doi:10.1177/0890334414526950.

233. Hauner, H, Much, D, Vollhardt, C, Brunner, S, Schmid, D, Sedlmeier, EM, Heimberg, E, Schuster, T, Zimmermann, A, Schneider, KT, Bader, BL, Amann-Gassner, U. Effect of reducing the n-6:n-3 long-chain PUFA ratio during pregnancy and lactation on infant adipose tissue growth within the first year of life: an open-label randomized controlled trial. Am J Clin Nutr. 2012. 95:383-94. doi:10.3945/ajen.111.022590.

234. Hauner, H, Vollhardt, C, Schneider, KT, Zimmermann, A, Schuster, T, Amann-Gassner, U.

Outcome; No Results The impact of nutritional fatty acids during pregnancy and lactation on early human adipose tissue development. Rationale and design of the INFAT study. Ann Nutr Metab. 2009. 54:97-103. doi:10.1159/000209267.

235. Hawkins, M, Hosker, M, Marcus, BH, Rosal, MC, Braun, B, Stanek, EJ, 3rd, Markenson, G, Chasan-Taber, L. A pregnancy lifestyle intervention to prevent gestational diabetes risk factors in overweight Hispanic women: a feasibility randomized controlled trial. Diabet Med. 2015. 32:108-15. doi:10.1111/dme.12601.

236. Heery, E, Kelleher, CC, Wall, PG, McAuliffe, FM. Prediction of gestational weight gain - a biopsychosocial model. Public Health Nutr. 2015. 18:1488-98. doi:10.1017/s1368980014001815.

237. Heery, E, Wall, PG, Kelleher, CC, McAuliffe, FM. Effects of dietary restraint and weight gain attitudes on gestational weight gain. Appetite. 2016. 107:501-510. doi:10.1016/j.appet.2016.08.103.

Intervention/Exposure

Intervention/Exposure

Intervention/Exposure 
238. Hernandez, TL, Van Pelt, RE, Anderson, MA, Reece, MS, Reynolds, RM, de la Houssaye, BA, Heerwagen, M, Donahoo, WT, Daniels, LJ, Chartier-Logan, C, Janssen, RC, Friedman, JE, Barbour, LA. Women With Gestational Diabetes Mellitus Randomized to a Higher-Complex Carbohydrate/Low-Fat Diet Manifest Lower Adipose Tissue Insulin Resistance, Inflammation, Glucose, and Free Fatty Acids: A Pilot Study. Diabetes Care. 2016. 39:39-42. doi:10.2337/dc15-0515.

239. Herrick, K, Phillips, DI, Haselden, S, Shiell, AW, Campbell-Brown, M, Godfrey, KM. Maternal consumption of a high-meat, low-carbohydrate diet in late pregnancy: relation to adult cortisol concentrations in the offspring. J Clin Endocrinol Metab. 2003. 88:3554-60. doi:10.1210/jc.2003-030287.

240. Hill, AJ, Cairnduff, V, McCance, DR. Nutritional and clinical associations of food cravings in pregnancy. J Hum Nutr Diet. 2016. 29:281-9. doi:10.1111/jhn.12333.

241. Hill, AJ, McCance, DR. Anthropometric and nutritional associations of food cravings in pregnancy. Pregnancy Hypertens. 2014. 4:235. doi:10.1016/j.preghy.2014.03.018.

242. Hinkle, S, Li, M, Grewal, J, Yisahak, S, Grantz, K, Ajjarapu, A, Zhang, C. Beverage Intake in U.S. Women Across Pregnancy and Gestational Diabetes Risk (P11-010-19). Curr Dev Nutr. 2019. 3. doi:10.1093/cdn/nzz048.P11-010-19.

243. Hinkle, SN, Rawal, S, Bjerregaard, AA, Halldorsson, TI, Li, M, Ley, SH, Wu, J, Zhu, Y, Chen, L, Liu, A, Grunnet, LG, Rahman, ML, Kampmann, FB, Mills, JL, Olsen, SF, Zhang, C. A prospective study of artificially sweetened beverage intake and cardiometabolic health among women at high risk. Am J Clin Nutr. 2019. doi:10.1093/ajcn/nqz094.

244. Hinton, PS, Olson, CM. Postpartum exercise and food intake: the importance of behaviorspecific self-efficacy. J Am Diet Assoc. 2001. 101:1430-7. doi:10.1016/s00028223(01)00345-5.

245. Horan, M, Donnelly, J, McGowan, C, Gibney, E, McAuliffe, F. The association between maternal nutrition and lifestyle during pregnancy and 2-year-old offspring adiposity: analysis from the ROLO study. Journal of Public Health (09431853). 2016. 24:427-436. doi:10.1007/s10389-016-0740-9.

246. Horan, MK, McGowan, CA, Gibney, ER, Donnelly, JM, McAuliffe, FM. Maternal low glycaemic index diet, fat intake and postprandial glucose influences neonatal adiposity-secondary analysis from the ROLO study. Nutr J. 2014. 13:78. doi:10.1186/1475-289113-78.

247. Horan, MK, McGowan, CA, Gibney, ER, Donnelly, JM, McAuliffe, FM. Maternal diet and weight at 3 months postpartum following a pregnancy intervention with a low glycaemic index diet: results from the ROLO randomised control trial. Nutrients. 2014. 6:2946-55. doi:10.3390/nu6072946.

Intervention/Exposure; Outcome

Intervention/Exposure; Outcome

Study Design

\section{Abstract}

Intervention/Exposure

Intervention/Exposure

Intervention/Exposure; Population

Intervention/Exposure; Comparator

Intervention/Exposure 
248. Hronek, M, Doubkova, P, Hrnciarikova, D, Zadak, Z. Dietary intake of energy and nutrients in relation to resting energy expenditure and anthropometric parameters of Czech pregnant women. Eur J Nutr. 2013. 52:117-25. doi:10.1007/s00394-011-0293-1.

249. Hronek, M, Doubkova, $P$, Tosner, J, Zadak, Z. Prediction of nutritive intake energy and substrates of Czech pregnant women. Nutrition. 2011. 27:1118-24. doi:10.1016/j.nut.2010.12.008.

250. Hsu, WY, Wu, CH, Hsieh, CT, Lo, HC, Lin, JS, Kao, MD. Low body weight gain, low white blood cell count and high serum ferritin as markers of poor nutrition and increased risk for preterm delivery. Asia Pac J Clin Nutr. 2013. 22:90-9. doi:10.6133/apjcn.2013.22.1.05.

251. Hu, G, Tian, H, Zhang, F, Liu, H, Zhang, C, Zhang, S, Wang, L, Liu, G, Yu, Z, Yang, X, Qi, L, Zhang, C, Wang, H, Li, M, Leng, J, Li, Y, Dong, L, Tuomilehto, J. Tianjin Gestational Diabetes Mellitus Prevention Program: study design, methods, and 1-year interim report on the feasibility of lifestyle intervention program. Diabetes Res Clin Pract. 2012. 98:50817. doi:10.1016/j.diabres.2012.09.015.

252. Huang, RC, Silva, D, Beilin, L, Neppe, C, Mackie, KE, Roffey, E, Gibson, LY, D'Vaz, N, Christian, H, Reid, CM, Prescott, SL. Feasibility of conducting an early pregnancy diet and lifestyle e-health intervention: the Pregnancy Lifestyle Activity Nutrition (PLAN) project. J Dev Orig Health Dis. 2019. :1-13. doi:10.1017/s2040174419000400.

253. Huang, Z, Li, N, Hu, YM. Dietary patterns and their effects on postpartum weight retention Study Design of lactating women in south central China. Nutrition. 2019. 67-68:110555. doi:10.1016/j.nut.2019.110555.

254. Huddy, RL, Torres, SJ, Milte, CM, McNaughton, SA, Teychenne, M, Campbell, KJ. Higher Adherence to the Australian Dietary Guidelines Is Associated with Better Mental Health Status among Australian Adult First-Time Mothers. J Acad Nutr Diet. 2016. 116:14061412. doi:10.1016/j.jand.2016.01.010.

255. Huh, SY, Rifas-Shiman, SL, Kleinman, KP, Rich-Edwards, JW, Lipshultz, SE, Gillman, MW. Maternal protein intake is not associated with infant blood pressure. Int J Epidemiol. 2005. 34:378-84. doi:10.1093/ije/dyh373.

256. Hui, A, Back, L, Ludwig, S, Gardiner, P, Sevenhuysen, G, Dean, H, Sellers, E, McGavock, J, Morris, M, Bruce, S, Murray, R, Shen, GX. Lifestyle intervention on diet and exercise reduced excessive gestational weight gain in pregnant women under a randomised controlled trial. Bjog. 2012. 119:70-7. doi:10.1111/j.1471-0528.2011.03184.x.

257. Hui, A, Back, L, Ludwig, S, Gardiner, P, Sevenhuysen, G, Dean, H, Sellers, E, McGavock J, Morris, M, Bruce, S, etal, . Exercise and dietary intervention increases physical activity, promotes healthy diet and reduces excessive gestational weight gain in pregnant women: a randomized controlled trial in Urban community. Diabetes. 2011. 60:A351-. doi:10.2337/db11-868-1281. 
258. Hui, A, Back, L, Ludwig, S, Gardiner, P, Sevenhuysen, G, Dean, H, Sellers, E, McGavock, J, Morris, M, Bruce, S, etal, . Lifestyle intervention on diet and exercise reduced excessive gestational weight gain in pregnant women under a randomized controlled trial. Obstetrical \& gynecological survey. 2012. 67:263-264. doi:10.1097/OGX.0b013e3182561861.

259. Hui, AL, Back, L, Ludwig, S, Gardiner, P, Sevenhuysen, G, Dean, HJ, Sellers, E, McGavock, J, Morris, M, Jiang, D, Shen, GX. Effects of lifestyle intervention on dietary intake, physical activity level, and gestational weight gain in pregnant women with different pre-pregnancy Body Mass Index in a randomized control trial. BMC Pregnancy Childbirth. 2014. 14:331. doi:10.1186/1471-2393-14-331.

260. Hui, AL, Back, L, Reid, A, Sevenhuysen, G, Ludwig, S, Dean, H, Sellers, E, McGavock, J, Morris, M, Shen, G. Effects of physical activity and dietary intakes on weight gain of pregnant women with normal and above normal prepregnancy weight. Canadian journal of diabetes. 2012. 36:S8-

261. Huseinovic, E, Bertz, F, Brekke, HK, Winkvist, A. Two-year follow-up of a postpartum weight loss intervention: Results from a randomized controlled trial. Maternal and Child Nutrition. 2018. 14. doi:10.1111/mcn.12539.

262. Huseinovic, E, Bertz, F, Leu Agelii, M, Hellebo Johansson, E, Winkvist, A, Brekke, HK Effectiveness of a weight loss intervention in postpartum women: results from a randomized controlled trial in primary health care. Am J Clin Nutr. 2016. 104:362-70. doi:10.3945/ajcn.116.135673.

263. Huseinovic, E, Ohlin, M, Winkvist, A, Bertz, F, Sonesson, U, Brekke, HK. Does diet intervention in line with nutrition recommendations affect dietary carbon footprint? Results from a weight loss trial among lactating women. Eur J Clin Nutr. 2017. 71:1241-1245. doi:10.1038/ejcn.2017.63.

264. Huseinovic, E, Winkvist, A, Bertz, F, Berteus Forslund, H, Brekke, HK. Eating frequency, energy intake and body weight during a successful weight loss trial in overweight and obese postpartum women. Eur J Clin Nutr. 2014. 68:71-6. doi:10.1038/ejcn.2013.200.

265. Huseinovic, E, Winkvist, A, Bertz, F, Brekke, HK. Changes in food choice during a successful weight loss trial in overweight and obese postpartum women. Obesity (Silver Spring). 2014. 22:2517-23. doi:10.1002/oby.20895.

266. Huseinovic, E, Winkvist, A, Bertz, F, Hellebo Johansson, E, Brekke, HK. Dietary assessment among women with overweight and obesity in early postpartum. J Hum Nutr Diet. 2016. 29:411-7. doi:10.1111/jhn.12350.

267. Hutchison, SM, Masse, LC, Glier, MB, Brain, U, Devlin, AM, Oberlander, TF. Impact of Prenatal Selective Serotonin Reuptake Inhibitor Antidepressant Exposure and Maternal Mood on Physical Activity, Dietary Intake, and Markers of Adiposity at Age 6 Years. J Dev Behav Pediatr. 2019. 40:266-274. doi:10.1097/dbp.0000000000000658.

\section{Editorial comment}

Intervention/Exposure; Exercise co-intervention

Intervention/Exposure; Population

Intervention/Exposure

Intervention/Exposure; Population

Intervention/Exposure

Outcome

Study Design; Outcome

Intervention/Exposure 
268. Huvinen, E, Koivusalo, SB, Meinila, J, Valkama, A, Tiitinen, A, Rono, K, Stach-Lempinen, B, Eriksson, JG. Effects of a Lifestyle Intervention During Pregnancy and First Postpartum Year: Findings From the RADIEL Study. J Clin Endocrinol Metab. 2018. 103:1669-1677. doi:10.1210/jc.2017-02477.

269. IImonen, J, Isolauri, E, Poussa, T, Laitinen, K. Impact of dietary counselling and probiotic intervention on maternal anthropometric measurements during and after pregnancy: a randomized placebo-controlled trial. Clin Nutr. 2011. 30:156-64. doi:10.1016/j.clnu.2010.09.009.

270. Jaakkola, J, Hakala, $P$, Isolauri, E, Poussa, T, Laitinen, K. Eating behavior influences diet, weight, and central obesity in women after pregnancy. Nutrition. 2013. 29:1209-13. doi:10.1016/j.nut.2013.03.008.

271. Jaakkola, J, Isolauri, E, Poussa, T, Laitinen, K. Benefits of repeated individual dietary counselling in long-term weight control in women after delivery. Matern Child Nutr. 2015. 11:1041-8. doi:10.1111/mcn.12115.

272. Jackson, RA, Stotland, NE, Caughey, $A B$, Gerbert, B. Improving diet and exercise in pregnancy with Video Doctor counseling: a randomized trial. Patient Educ Couns. 2011. 83:203-9. doi:10.1016/j.pec.2010.05.019.

273. Janmohamed, R, Montgomery-Fajic, E, Sia, W, Germaine, D, Wilkie, J, Khurana, R, Nerenberg, KA. Cardiovascular risk reduction and weight management at a hospitalbased postpartum preeclampsia clinic. J Obstet Gynaecol Can. 2015. 37:330-337. doi:10.1016/s1701-2163(15)30283-8.

274. Jansson, N, Nilsfelt, A, Gellerstedt, M, Wennergren, M, Rossander-Hulthen, L, Powell, TL, Jansson, T. Maternal hormones linking maternal body mass index and dietary intake to birth weight. Am J Clin Nutr. 2008. 87:1743-9. doi:10.1093/ajcn/87.6.1743.

275. Jardí, C, Aparicio, E, Bedmar, C, Aranda, N, Abajo, S, March, G, Basora, J, Arija, V. Food consumption during pregnancy and post-partum. ECLIPSES study. Nutrients. 2019. 11. doi:10.3390/nu11102447.

276. Jaruratanasirikul, $S$, Sangsupawanich, $P$, Koranantakul, $O$, Chanvitan, $P$, Sriplung, $H$, Patanasin, T. Influence of maternal nutrient intake and weight gain on neonatal birth weight: a prospective cohort study in southern Thailand. J Matern Fetal Neonatal Med. 2009. 22:1045-50. doi:10.3109/14767050903019668.

277. Jedrychowski, W, Perera, FP, Tang, D, Stigter, L, Mroz, E, Flak, E, Spengler, J, BudzynMrozek, D, Kaim, I, Jacek, R. Impact of barbecued meat consumed in pregnancy on birth outcomes accounting for personal prenatal exposure to airborne polycyclic aromatic hydrocarbons: Birth cohort study in Poland. Nutrition. 2012. 28:372-7. doi:10.1016/j.nut.2011.07.020.
Outcome; Comparator

Population; Outcome

Intervention/Exposure; Outcome

Intervention/Exposure; Population

Intervention/Exposure; Comparator

Intervention/Exposure

Outcome; Comparator

Outcome

Intervention/Exposure; Outcome

Intervention/Exposure; Outcome 
278. Jiang, F, Li, Y, Xu, P, Li, J, Chen, X, Yu, H, Gao, B, Xu, B, Li, X, Chen, W. The efficacy of the Dietary Approaches to Stop Hypertension diet with respect to improving pregnancy outcomes in women with hypertensive disorders. J Hum Nutr Diet. 2019. doi:10.1111/jhn.12654.

279. Jing, W, Huang, $Y$, Liu, $X$, Luo, B, Yang, Y, Liao, S. The effect of a personalized Comparator; Exercise co-intervention intervention on weight gain and physical activity among pregnant women in China. Int $\mathrm{J}$ Gynaecol Obstet. 2015. 129:138-41. doi:10.1016/j.jgo.2014.11.014.

280. Juhl, M, Madsen, M, Andersen, AMN, Andersen, PK, Olsen, J. Distribution and predictors of exercise habits among pregnant women in the Danish National Birth Cohort. Scandinavian Journal of Medicine \& Science in Sports. 2012. 22:128-138. doi:10.1111/j.1600-0838.2010.01125.x.

281. Jung, YM, Choi, MJ. Nutrient Intake according to Weight Gain during Pregnancy, Job Status, and Household Income. Clin Nutr Res. 2017. 6:27-37. doi:10.7762/cnr.2017.6.1.27.

282. Kac, G, Benicio, MH, Velasquez-Melendez, G, Valente, JG, Struchiner, CJ. Breastfeeding and postpartum weight retention in a cohort of Brazilian women. Am J Clin Nutr. 2004. 79:487-93. doi:10.1093/ajcn/79.3.487.

283. Karamanos, B, Thanopoulou, A, Anastasiou, E, Assaad-Khalil, S, Albache, N, Bachaoui, M, Slama, CB, El Ghomari, H, Jotic, A, Lalic, N, Lapolla, A, Saab, C, Marre, M, Vassallo, J, Savona-Ventura, C. Relation of the Mediterranean diet with the incidence of gestational diabetes. Eur J Clin Nutr. 2014. 68:8-13. doi:10.1038/ejcn.2013.177.

284. Karayiannis, D, Kontogianni, MD, Mendorou, C, Mastrominas, M, Yiannakouris, N. Adherence to the Mediterranean diet and IVF success rate among non-obese women attempting fertility. Hum Reprod. 2018. 33:494-502. doi:10.1093/humrep/dey003.

285. Kay, MC, Wasser, H, Adair, LS, Thompson, AL, Siega-Riz, AM, Suchindran, CM, Bentley, ME. Consumption of key food groups during the postpartum period in low-income, nonHispanic black mothers. Appetite. 2017. 117:161-167. doi:10.1016/j.appet.2017.06.023.

286. Keller, C, Todd, M, Ainsworth, B, Records, K, Vega-Lopez, S, Permana, P, Coonrod, D, Williams, AN. Overweight, Obesity, and Neighborhood Characteristics among Postpartum Latinas. Journal of Obesity. 2013. 2013:1-8. doi:2013/916468.

287. Kennedy, RAK, Turner, MJ. Development of a novel Periconceptual Nutrition Score (PENS) to examine the relationship between maternal dietary quality and fetal growth. Early Human Development. 2019. 132:6-12. doi:10.1016/j.earlhumdev.2019.03.004.

288. Khoury, J, Henriksen, T, Christophersen, B, Tonstad, S. Effect of a cholesterol-lowering diet on maternal, cord, and neonatal lipids, and pregnancy outcome: a randomized clinical trial. American journal of obstetrics and gynecology. 2005. 193:1292-1301. doi:10.1016/j.ajog.2005.05.016.

Outcome

Study Design; Intervention/Exposure

Intervention/Exposure

Comparator; Country

Population; Outcome

Intervention/Exposure; Outcome

Intervention/Exposure; Outcome

\section{Outcome}

Intervention/Exposure 
289. Kim, JH, Lee, SJ, Kim, SY, Choi, G, Lee, JJ, Kim, HJ, Kim, S, Park, J, Moon, HB, Choi, K, $\mathrm{Kim}, \mathrm{S}$, Choi, SR. Association of food consumption during pregnancy with mercury and lead levels in cord blood. Sci Total Environ. 2016. 563-564:118-24. doi:10.1016/j.scitotenv.2016.04.082.

290. Kim, MJ, Kim, TH, Park, Y, Lee, HH, Kim, JM, Lim, H, Hwang, SY. A study of the dietary Outcome; Comparator intakes by the pre-pregnancy body mass index in pregnant women. Clin Exp Obstet Gynecol. 2017. 44:27-29.

291. Kim, O, Ahn, Y, Lee, HY, Jang, HJ, Kim, S, Lee, JE, Jung, H, Cho, E, Lim, JY, Kim, MJ, Willett, WC, Chavarro, JE, Park, HY. The Korea Nurses' Health Study: A Prospective Cohort Study. J Womens Health (Larchmt). 2017. 26:892-899. doi:10.1089/jwh.2016.6048.

292. Kim, SH, Kim, MY, Yang, JH, Park, SY, Yim, CH, Han, KO, Yoon, HK, Park, S. Nutritional risk factors of early development of postpartum prediabetes and diabetes in women with gestational diabetes mellitus. Nutrition. 2011. 27:782-8. doi:10.1016/j.nut.2010.08.019.

293. Kinnunen, T, Puhkala, J, Raitanen, J, Ahonen, S, Aittasalo, M, Virtanen, SM, Luoto, R. Effects of dietary counselling on food habits and dietary intake of Finnish pregnant women at increased risk for gestational diabetes - a secondary analysis of a cluster-randomized controlled trial. Maternal \& Child Nutrition. 2014. 10:184-197. doi:10.1111/j.17408709.2012.00426.x.

294. Kinnunen, TI, Pasanen, M, Aittasalo, M, Fogelholm, M, Weiderpass, E, Luoto, R. Reducing postpartum weight retention--a pilot trial in primary health care. Nutr J. 2007. 6:21. doi:10.1186/1475-2891-6-21.

295. Kiyak Caglayan, E, Engin-Ustun, Y, Sari, N, Gocmen, AY, Polat, MF. The effects of prolonged fasting on the levels of adiponectin, leptin, apelin, and omentin in pregnant women. J Obstet Gynaecol. 2016. 36:555-8. doi:10.3109/01443615.2015.1103716.

296. Kiziltan, G, Karabudak, E, Tuncay, G, Avsar, F, Tuncay, P, Mungan, O, Meral, P. Dietary intake and nutritional status of Turkish pregnant women during Ramadan. Saudi Med J. 2005. 26:1782-7.

297. Kizirian, N, Garnett, S, Markovic, T, Ross, G, Muirhead, R, Brodie, S, Petocz, P, BrandMiller, J. Maternal diet and infant body composition in women at risk of gestational diabetes mellitus. Obesity research \& clinical practice. 2014. 8:55doi:10.1016/j.orcp.2014.10.102.

298. Kizirian, NV, Markovic, TP, Muirhead, R, Brodie, S, Garnett, SP, Louie, JC, Petocz, P, Ross, GP, Brand-Miller, JC. Macronutrient Balance and Dietary Glycemic Index in Pregnancy Predict Neonatal Body Composition. Nutrients. 2016. 8. doi:10.3390/nu8050270. 
299. Knudsen, VK, Heitmann, BL, Halldorsson, TI, Sorensen, TI, Olsen, SF. Maternal dietary glycaemic load during pregnancy and gestational weight gain, birth weight and postpartum weight retention: a study within the Danish National Birth Cohort. Br J Nutr. 2013. 109:1471-8. doi:10.1017/s0007114512003443.

300. Kombol, P. ILCA's Inside Track: a resource for breastfeeding mothers. Breastfeeding after weight loss surgery. J Hum Lact. 2008. 24:341-2. doi:10.1177/08903344080240031801.

301. Kominiarek, MA. A Survey of Health Behaviors in Minority Women in Pregnancy: The Influence of Body Mass Index. Women's Health Issues. 2014. 24:e291-5. doi:10.1016/j.whi.2014.02.007.

302. Kong, A, Odoms-Young, AM, Schiffer, LA, Berbaum, ML, Porter, SJ, Blumstein, L, Fitzgibbon, ML. Racial/Ethnic Differences in Dietary Intake among WIC Families Prior to Food Package Revisions. Journal of Nutrition Education \& Behavior. 2013. 45:39-46. doi:10.1016/j.jneb.2012.04.014.

303. Korpi-Hyovalti, E, Schwab, U, Laaksonen, DE, Linjama, H, Heinonen, S, Niskanen, L. Effect of intensive counselling on the quality of dietary fats in pregnant women at high risk of gestational diabetes mellitus. Br J Nutr. 2012. 108:910-7. doi:10.1017/s0007114511006118.

304. Koutelidakis, AE, Alexatou, O, Kousaiti, S, Gkretsi, E, Vasios, G, Sampani, A, Tolia, M, Kiortsis, DN, Giaginis, C. Higher adherence to Mediterranean diet prior to pregnancy is associated with decreased risk for deviation from the maternal recommended gestational weight gain. Int J Food Sci Nutr. 2018. 69:84-92. doi:10.1080/09637486.2017.1330403.

305. Krawczyk, P, Sioma-Markowska, U, Nowak-Brzezińska, A, Skrzypulec-Plinta, V, Kubiak, E. Relationship of eating habits of pregnant women and daily delivery of nutrients as well as their influence on the course of pregnancy and condition of the neonate. Ginekologia i Poloznictwo. 2016. 42:33-42.

306. Kubota, K, Itoh, H, Tasaka, M, Naito, H, Fukuoka, Y, Muramatsu Kato, K, Kohmura, YK Sugihara, K, Kanayama, N. Changes of maternal dietary intake, bodyweight and fetal growth throughout pregnancy in pregnant Japanese women. J Obstet Gynaecol Res. 2013. 39:1383-90. doi:10.1111/jog.12070.

307. Kunath, J, Günther, J, Rauh, K, Hoffmann, J, Stecher, L, Rosenfeld, E, Kick, L, Ulm, K, Hauner, $\mathrm{H}$. Effects of a lifestyle intervention during pregnancy to prevent excessive gestational weight gain in routine care - the cluster-randomised GeliS trial. BMC Medicine. 2019. 17. doi:10.1186/s12916-018-1235-z.

308. Lagiou, P, Lagiou, A, Samoli, E, Hsieh, CC, Adami, HO, Trichopoulos, D. Diet during pregnancy and levels of maternal pregnancy hormones in relation to the risk of breast cancer in the offspring. Eur J Cancer Prev. 2006. 15:20-6.

\section{Study Design}

Study Design; Intervention/Exposure

Study Design; Outcome

Intervention/Exposure

Study Design

\section{Outcome}

\section{Intervention/Exposure}

\section{Intervention/Exposure}


309. Lagiou, P, Samoli, E, Lipworth, L, Lagiou, A, Fang, F, Rossi, M, Xu, B, Yu, GP, Adami, $\mathrm{HO}$, Hsieh, CC, Trichopoulos, D. Energy intake during pregnancy in relation to offspring gender by maternal height. Eur J Epidemiol. 2011. 26:39-44. doi:10.1007/s10654-0109528-3.

310. Lagiou, $\mathrm{P}$, Tamimi, RM, Mucci, LA, Adami, HO, Hsieh, CC, Trichopoulos, D. Diet during Intervention/Exposure; Comparator pregnancy in relation to maternal weight gain and birth size. Eur J Clin Nutr. 2004. 58:231-7. doi:10.1038/sj.ejcn.1601771.

311. Lai, JS, Soh, SE, Loy, SL, Colega, M, Kramer, MS, Chan, JKY, Tan, TC, Shek, LPC, Yap, FKP, Tan, KH, Godfrey, KM, Chong, YS, Chong, MFF. Macronutrient composition and food groups associated with gestational weight gain: the GUSTO study. Eur J Nutr. 2019 58:1081-1094. doi:10.1007/s00394-018-1623-3.

312. Lamichhane, DK, Leem, JH, Kim, HC, Lee, JY, Park, MS, Jung, DY, Ko, JK, Ha, M, Kim, $\mathrm{Y}$, Hong, YC, Ha, EH. Impact of prenatal exposure to polycyclic aromatic hydrocarbons from maternal diet on birth outcomes: a birth cohort study in Korea. Public Health Nutr. 2016. 19:2562-71. doi:10.1017/s1368980016000550.

313. Lamyian, M, Hosseinpour-Niazi, $S$, Mirmiran, $P$, Moghaddam Banaem, L, Goshtasebi, $A$, Azizi, F. Pre-Pregnancy Fast Food Consumption Is Associated with Gestational Diabetes Mellitus among Tehranian Women. Nutrients. 2017. 9. doi:10.3390/nu9030216.

314. Laraia, B, Vinikoor-Imler, LC, Siega-Riz, AM. Food insecurity during pregnancy leads to Intervention/Exposure stress, disordered eating, and greater postpartum weight among overweight women. Obesity. 2015. 23:1303-11. doi:10.1002/oby.21075.

315. Laraia, BA, Adler, NE, Coleman-Phox, K, Vieten, C, Mellin, L, Kristeller, JL, Thomas, M, Stotland, NE, Lustig, RH, Dallman, MF, Hecht, FM, Bush, NR, de Groat, CL, Epel, E. Novel Interventions to Reduce Stress and Overeating in Overweight Pregnant Women: A Feasibility Study. Matern Child Health J. 2018. 22:670-678. doi:10.1007/s10995-0182435-z.

316. Laraia, BA, Bodnar, LM, Siega-Riz, AM. Pregravid body mass index is negatively associated with diet quality during pregnancy. Public Health Nutr. 2007. 10:920-6. doi:10.1017/s1368980007657991.

317. Laraia, BA, Siega-Riz, AM, Dole, N, London, E. Pregravid weight is associated with prior dietary restraint and psychosocial factors during pregnancy. Obesity (Silver Spring). 2009. 17:550-8. doi:10.1038/oby.2008.585.

318. Larranaga, I, Santa-Marina, L, Begiristain, H, Machon, M, Vrijheid, M, Casas, M, Tardon, A, Fernandez-Somoano, A, Llop, S, Rodriguez-Bernal, CL, Fernandez, MF. Socioeconomic inequalities in health, habits and self-care during pregnancy in Spain. Matern Child Health J. 2013. 17:1315-24. doi:10.1007/s10995-012-1134-4.

\section{Intervention/Exposure}

Intervention/Exposure

Intervention/Exposure

Intervention/Exposure

Outcome

Intervention/Exposure; Outcome

Intervention/Exposure; Outcome 
319. Larsen, SC, Angquist, L, Laurin, C, Morgen, CS, Jakobsen, MU, Paternoster, L, Smith, GD, Olsen, SF, Sorensen, TI, Nohr, EA. Association between Maternal Fish Consumption and Gestational Weight Gain: Influence of Molecular Genetic Predisposition to Obesity. PLoS One. 2016. 11:e0150105. doi:10.1371/journal.pone.0150105.

320. Latva-Pukkila, $U$, Isolauri, E, Laitinen, K. Dietary and clinical impacts of nausea and vomiting during pregnancy. J Hum Nutr Diet. 2010. 23:69-77. doi:10.1111/j.1365277X.2009.01019.x.

321. Lavie, M, Lavie, I, Maslovitz, S. Paleolithic diet during pregnancy-A potential beneficial effect on metabolic indices and birth weight. Eur J Obstet Gynecol Reprod Biol. 2019. 242:7-11. doi:10.1016/j.ejogrb.2019.08.013.

322. Leahy, K, Berlin, KS, Banks, GG, Bachman, J. The Relationship Between Intuitive Eating and Postpartum Weight Loss. Matern Child Health J. 2017. 21:1591-1597. doi:10.1007/s10995-017-2281-4.

323. Ledoux, T, Robinson, J, Sampson, M, Beasley, A. Effect of Intuitive Eating on Gestational Conference abstract Weight Gain. Journal of the Academy of Nutrition \& Dietetics. 2016. 116:A16-A16. doi:10.1016/j.jand.2016.06.041.

324. Lee, JI, Lee, JA, Lim, HS. Morning sickness reduces dietary diversity, nutrient intakes, and infant outcome of pregnant women. Nutrition Research. 2004. 24:531-540. doi:10.1016/j.nutres.2003.10.011.

325. Leslie, DA, Hesketh, KD, Campbell, KJ. Breastfeeding mothers consume more vegetables and a greater variety of fruits and vegetables than non-breastfeeding peers: The influence of socioeconomic position. Nutrition \& Dietetics. 2012. 69:84-90. doi:10.1111/j.17470080.2012.01584.x.

326. Lesser, MNR, Mauldin, K, Sawrey-Kubicek, L, Gildengorin, V, King, JC. The Type of Dietary Fat in an Isocaloric Breakfast Meal Does Not Modify Postprandial Metabolism in Overweight/Obese Pregnant Women. Nutrients. 2019. 11. doi:10.3390/nu11030490.

327. Li, S, Zhu, Y, Chavarro, JE, Bao, W, Tobias, DK, Ley, SH, Forman, JP, Liu, A, Mills, J, Bowers, K, Strom, M, Hansen, S, Hu, FB, Zhang, C. Healthful Dietary Patterns and the Risk of Hypertension Among Women With a History of Gestational Diabetes Mellitus: A Prospective Cohort Study. Hypertension. 2016. 67:1157-65. doi:10.1161/hypertensionaha.115.06747.

328. Li, YM, Shen, YD, Li, YJ, Xun, GL, Liu, H, Wu, RR, Xia, K, Zhao, JP, Ou, JJ. Maternal dietary patterns, supplements intake and autism spectrum disorders: A preliminary casecontrol study. Medicine (Baltimore). 2018. 97:e13902. doi:10.1097/md.0000000000013902.

\section{Study Design}

Study Design; Intervention/Exposure

Intervention/Exposure

Study Design; Outcome

\section{Intervention/Exposure; Outcome}

Population; Outcome

Study Design; Outcome 
329. Lignell, S, Winkvist, A, Bertz, F, Rasmussen, KM, Glynn, A, Aune, M, Brekke, HK.

Environmental organic pollutants in human milk before and after weight loss.

Chemosphere. 2016. 159:96-102. doi:10.1016/j.chemosphere.2016.05.077.

330. Lindsay, K, Buss, C, Entringer, S, Wadhwa, P. The Interplay Between Diet Quality and

Abstract

Pre-pregnancy Body Mass Index on Glycemic Parameters in Pregnancy: A Comparison of Various Diet Quality Scores (OR35-02-19). Curr Dev Nutr. 2019. 3. doi:10.1093/cdn/nzz048.OR35-02-19.

331. Lindsay, KL, Heneghan, C, McNulty, B, Brennan, L, McAuliffe, FM. Lifestyle and dietary

Population habits of an obese pregnant cohort. Matern Child Health J. 2015. 19:25-32. doi:10.1007/s10995-014-1491-2.

332. Linné, Y, Rössner, S. Interrelationships between weight development and weight retention in subsequent pregnancies: The SPAWN study. Acta Obstetricia et Gynecologica Scandinavica. 2003. 82:318-325. doi:10.1034/j.1600-0412.2003.00150.x.

333. Lioret, S, Cameron, AJ, McNaughton, SA, Crawford, D, Spence, AC, Hesketh, K,

Campbell, KJ. Association between maternal education and diet of children at 9 months is partially explained by mothers' diet. Matern Child Nutr. 2015. 11:936-47. doi:10.1111/mcn.12031.

334. Lipsmeyer, M, Diaz, E, Sims, C, Cleves, M, Shankar, K, Andres, A. Antenatal and Postnatal Factors Associated with Offspring Adiposity During the First Two Years of Life (FS18-08-19). Curr Dev Nutr. 2019. 3. doi:10.1093/cdn/nzz041.FS18-08-19.

335. Liu, K, Ye, K, Han, Y, Sheng, J, Jin, Z, Bo, Q, Hu, C, Hu, C, Li, L. Maternal and cord blood fatty acid patterns with excessive gestational weight gain and neonatal macrosomia. Asia Pac J Clin Nutr. 2017. 26:291-297. doi:10.6133/apjcn.012016.11.

336. Liu, MJ, Li, HT, Yu, LX, Xu, GS, Ge, H, Wang, LL, Zhang, YL, Zhou, YB, Li, Y, Bai, MX, Liu, JM. A correlation study of DHA dietary intake and plasma, erythrocyte and breast milk DHA concentrations in lactatingwomen from Coastland, Lakeland, and Inland areas of China. Nutrients. 2016. 8. doi:10.3390/nu8050312.

337. Lof, M, Hilakivi-Clarke, L, Sandin, SS, de Assis, S, Yu, W, Weiderpass, E. Dietary fat intake and gestational weight gain in relation to estradiol and progesterone plasma levels during pregnancy: a longitudinal study in Swedish women. BMC Womens Health. 2009. 9:10. doi:10.1186/1472-6874-9-10.

338. Lombard, CB, Deeks, AA, Ball, K, Jolley, D, Teede, HJ. Weight, physical activity and dietary behavior change in young mothers: short term results of the HeLP-her cluster randomized controlled trial. Nutr J. 2009. 8:17. doi:10.1186/1475-2891-8-17.

339. Long, VA, Martin, T, Janson-Sand, C. The Great Beginnings program: impact of a nutrition curriculum on nutrition knowledge, diet quality, and birth outcomes in pregnant and parenting teens. Journal of the American Dietetic Association. 2002. 102:S86-9. 
340. Lopez-Cepero, A, Nobel, L, Moore-Simas, T, Rosal, M. Maternal Diet Quality and Infant Growth Trajectories During the First Year of Life (OR35-07-19). Curr Dev Nutr. 2019. 3. doi:10.1093/cdn/nzz048.OR35-07-19.

341. Lopez-Olmedo, N, Hernandez-Cordero, S, Neufeld, LM, Garcia-Guerra, A, MejiaRodriguez, F, Mendez Gomez-Humaran, I. The Associations of Maternal Weight Change with Breastfeeding, Diet and Physical Activity During the Postpartum Period. Matern Child Health J. 2016. 20:270-80. doi:10.1007/s10995-015-1826-7.

342. Lotfi, MH, Fallahzadeh, $\mathrm{H}$, Rahmanian, $M$, Hosseinzadeh, M, Lashkardoost, $H$, Doaei, S, Gholamalizadeh, M, Hamedi, A. Association of food groups intake and physical activity with gestational diabetes mellitus in Iranian women. J Matern Fetal Neonatal Med. 2019. :1-6. doi:10.1080/14767058.2019.1579189.

343. Louie, JC, Markovic, TP, Perera, N, Foote, D, Petocz, P, Ross, GP, Brand-Miller, JC. A randomized controlled trial investigating the effects of a low-glycemic index diet on pregnancy outcomes in gestational diabetes mellitus. Diabetes Care. 2011. 34:2341-6. doi:10.2337/dc11-0985.

344. Louie, JCY, Markovic, TP, Ross, GP, Foote, D, Brand-Miller, JC. Effect of a low glycaemic index diet in gestational diabetes mellitus on post-natal outcomes after 3 months of birth: a pilot follow-up study. Maternal \& Child Nutrition. 2015. 11:409-414. doi:10.1111/mcn.12039.

345. Lovelady, CA, Stephenson, KG, Kuppler, KM, Williams, JP. The effects of dieting on food and nutrient intake of lactating women. J Am Diet Assoc. 2006. 106:908-12. doi:10.1016/j.jada.2006.03.007.

346. Lu, MS, Chen, QZ, He, JR, Wei, XL, Lu, JH, Li, SH, Wen, XX, Chan, FF, Chen, NN, Qiu, L, Outcome Mai, WB, Zhang, RF, Hu, CY, Xia, HM, Qiu, X. Maternal Dietary Patterns and Fetal Growth: A Large Prospective Cohort Study in China. Nutrients. 2016. 8. doi:10.3390/nu8050257.

347. Lundeen, E, Park, S, Baidal, JAW, Sharma, A, Blanck, HM. Prevalence of and Factors Associated with Sugar-sweetened Beverage Intake Among Women of Reproductive Age12 States and District of Columbia, 2017 (P16-022-19). Curr Dev Nutr. 2019. 3. doi:10.1093/cdn/nzz050.P16-022-19.

348. Luoto, R, Kharazmi, E, Saarinen, NM, Smeds, Al, Mäkelä, S, Fallah, M, Raitanen, J, Hilakivi-Clarke, L. Effect of dietary intervention on serum lignan levels in pregnant women - a controlled trial. Reproductive Health. 2010. 7:6p-6p. doi:10.1186/1742-4755-7-26.

349. Luoto, RM, Kinnunen, TI, Aittasalo, M, Ojala, K, Mansikkamaki, K, Toropainen, E, Kolu, P, Vasankari, T. Prevention of gestational diabetes: design of a cluster-randomized controlled trial and one-year follow-up. BMC Pregnancy Childbirth. 2010. 10:39. doi:10.1186/1471-2393-10-39.

Intervention/Exposure

Study Design; Intervention/Exposure

Health Status

Intervention/Exposure; Population

Intervention/Exposure; Outcome

Abstract

Intervention/Exposure; Outcome

Study Design; Intervention/Exposure 
350. Lyu, LC, Lo, CC, Chen, HF, Wang, CY, Liu, DM. A prospective study of dietary intakes and influential factors from pregnancy to postpartum on maternal weight retention in Taipei, Taiwan. Br J Nutr. 2009. 102:1828-37. doi:10.1017/s0007114509991243.

351. Ma, C, Shaw, GM, Scheuerle, AE, Canfield, MA, Carmichael, SL. Association of microtia

Outcome with maternal nutrition. Birth Defects Res A Clin Mol Teratol. 2012. 94:1026-32. doi:10.1002/bdra.23053.

352. Ma, L, Lu, Q, Ouyang, J, Huang, J, Huang, S, Jiao, C, Zhang, Z, Mao, L. How are maternal dietary patterns and maternal/fetal cytokines associated with birth weight? A path analysis. Br J Nutr. 2019. 121:1178-1187. doi:10.1017/s0007114519000382.

353. Ma, WJ, Huang, ZH, Huang, BX, Qi, BH, Zhang, YJ, Xiao, BX, Li, YH, Chen, L, Zhu, HL. Intensive low-glycaemic-load dietary intervention for the management of glycaemia and serum lipids among women with gestational diabetes: a randomized control trial. Public Health Nutr. 2015. 18:1506-13. doi:10.1017/s1368980014001992.

354. Mak, JKL, Pham, NM, Lee, AH, Tang, L, Pan, XF, Binns, CW, Sun, X. Dietary patterns during pregnancy and risk of gestational diabetes: a prospective cohort study in Western China. Nutr J. 2018. 17:107. doi:10.1186/s12937-018-0413-3.

355. Makela, J, Lagstrom, H, Kaljonen, A, Simell, O, Niinikoski, H. Hyperglycemia and lower diet quality in pregnant overweight women and increased infant size at birth and at 13 months of age--STEPS study. Early Hum Dev. 2013. 89:439-44. doi:10.1016/j.earlhumdev.2013.01.007.

356. Malek, L, Makrides, M. 2.8 Nutrition in pregnancy and lactation. World Rev Nutr Diet. 2015. 113:127-33. doi:10.1159/000367872.

357. Man, YB, Chow, KL, Xing, GH, Chan, JKY, Wu, SC, Wong, MH. A pilot study on health risk assessment based on body loadings of PCBs of lactating mothers at Taizhou, China, the world's major site for recycling transformers. Environ Pollut. 2017. 227:364-371. doi:10.1016/j.envpol.2017.04.069.

358. Mannion, CA, Gray-Donald, K, Johnson-Down, L, Koski, KG. Lactating women restricting milk are low on select nutrients. J Am Coll Nutr. 2007. 26:149-55.

359. Markhus, MW, Graff, IE, Dahl, L, Seldal, CF, Skotheim, S, Braarud, HC, Stormark, KM, Malde, MK. Establishment of a seafood index to assess the seafood consumption in pregnant women. Food \& Nutrition Research. 2013. 57:1-11. doi:10.3402/fnr.v57i0.19272.

360. Markovic, TP, Muirhead, R, Overs, S, Kizirian, N, Louie, J, Sweeting, A, Petocz, P, Denyer, G, Hyett, J, Ross, GP, et al. Predictors of birthweight in women at high risk of gestational diabetes mellitus. Obesity research and clinical practice. 2013. 7:e3-e4.

Outcome

Intervention/Exposure doi:10.1016/j.orcp.2013.12.505. 
361. Markovic, TP, Muirhead, R, Overs, S, Ross, GP, Louie, JC, Kizirian, N, Denyer, G, Petocz, P, Hyett, J, Brand-Miller, JC. Randomized Controlled Trial Investigating the Effects of a Low-Glycemic Index Diet on Pregnancy Outcomes in Women at High Risk of Gestational Diabetes Mellitus: The GI Baby 3 Study. Diabetes Care. 2016. 39:31-8. doi:10.2337/dc150572.

362. Marshburn, MK. Helping Obese Pregnant Women Achieve Healthy Weight Gain: Is Provider Intervention Feasible? Helping Obese Pregnant Women Achieve Healthy Weight Gain: Is Provider Intervention Feasible? 2017. :1-1.

363. Martin, CL, Siega-Riz, AM, Sotres-Alvarez, D, Robinson, WR, Daniels, JL, Perrin, EM, Stuebe, AM. Maternal Dietary Patterns during Pregnancy Are Associated with Child Growth in the First 3 Years of Life. J Nutr. 2016. 146:2281-2288. doi:10.3945/jn.116.234336.

364. Martin, CL, Siega-Riz, AM, Sotres-Alvarez, D, Robinson, WR, Daniels, JL, Perrin, EM, Stuebe, AM. Maternal Dietary Patterns are Associated with Lower Levels of Cardiometabolic Markers during Pregnancy. Paediatr Perinat Epidemiol. 2016. 30:246-55. doi:10.1111/ppe.12279.

365. Martin, J, MacDonald-Wicks, L, Hure, A, Smith, R, Collins, CE. Reducing postpartum weight retention and improving breastfeeding outcomes in overweight women: a pilot randomised controlled trial. Nutrients. 2015. 7:1464-79. doi:10.3390/nu7031464.

366. Martinez-Galiano, JM, Olmedo-Requena, R, Barrios-Rodriguez, R, Amezcua-Prieto, C, Bueno-Cavanillas, A, Salcedo-Bellido, I, Jimenez-Moleon, JJ, Delgado-Rodriguez, M. Effect of Adherence to a Mediterranean Diet and Olive Oil Intake during Pregnancy on Risk of Small for Gestational Age Infants. Nutrients. 2018. 10. doi:10.3390/nu10091234.

367. Martins, AP, Benicio, MH. Influence of dietary intake during gestation on postpartum weight retention. Rev Saude Publica. 2011. 45:870-7. doi:10.1590/s003489102011005000056.

368. Martins, MC, Trujillo, J, Freitas-Vilela, AA, Farias, DR, Rosado, EL, Struchiner, CJ, Kac, G. Outcome Associations between obesity candidate gene polymorphisms (fat mass and obesityassociated (FTO), melanocortin-4 receptor (MC4R), leptin (LEP) and leptin receptor (LEPR)) and dietary intake in pregnant women. Br J Nutr. 2018. 120:454-463. doi:10.1017/s0007114518001423.

369. Maslova, E, Halldorsson, TI, Astrup, A, Olsen, SF. Dietary protein-to-carbohydrate ratio and added sugar as determinants of excessive gestational weight gain: a prospective cohort study. BMJ Open. 2015. 5:e005839. doi:10.1136/bmjopen-2014-005839.

370. Maslova, E, Rytter, D, Bech, BH, Henriksen, TB, Olsen, SF, Halldorsson, TI. Maternal intake of fat in pregnancy and offspring metabolic health - A prospective study with 20 years of follow-up. Clin Nutr. 2016. 35:475-483. doi:10.1016/j.clnu.2015.03.018.

\section{Dissertation}

Intervention/Exposure; Outcome

\section{Outcome}

Intervention/Exposure

Study Design; Outcome

Population

Inte

Intervention/Exposure

Intervention/Exposure; Outcome 
371. Maugeri, A, Barchitta, M, Agrifoglio, O, Favara, G, La Mastra, C, La Rosa, MC, Magnano San Lio, R, Panella, M, Cianci, A, Agodi, A. The impact of social determinants and lifestyles on dietary patterns during pregnancy: evidence from the "Mamma \& Bambino" study. Ann Ig. 2019. 31:81-89. doi:10.7416/ai.2019.2280.

372. McGiveron, A, Foster, S, Pearce, J, Taylor, MA, McMullen, S, Langley-Evans, SC. Limiting antenatal weight gain improves maternal health outcomes in severely obese pregnant women: findings of a pragmatic evaluation of a midwife-led intervention. J Hum Nutr Diet. 2015. 28 Suppl 1:29-37. doi:10.1111/jhn.12240.

373. McGowan, CA, Curran, S, McAuliffe, FM. Relative validity of a food frequency questionnaire to assess nutrient intake in pregnant women. Journal of Human Nutrition \& Dietetics. 2014. :167-174. doi:10.1111/jhn.12120.

374. McGowan, CA, McAuliffe, FM. Maternal dietary patterns and associated nutrient intakes during each trimester of pregnancy. Public Health Nutr. 2013. 16:97-107. doi:10.1017/s1368980012000997.

375. McGowan, CA, Walsh, JM, Byrne, J, Curran, S, McAuliffe, FM. The influence of a low glycemic index dietary intervention on maternal dietary intake, glycemic index and gestational weight gain during pregnancy: a randomized controlled trial. Nutr J. 2013. 12:140. doi:10.1186/1475-2891-12-140.

376. McGuire, E. Breastfeeding and high maternal body mass index. Breastfeed Rev. 2013. 21:7-14.

377. McGurk, P, Hill, AJ, McCance, DR. An investigation of dietary intake of pregnant women in the third trimester in Northern Ireland. Journal of Human Nutrition \& Dietetics. 2011. 24:293-294. doi:10.1111/j.1365-277X.2011.01175_22.x.

378. Mcllvride, S, Nikolova, V, Fan, HM, McDonald, JAK, Wahlstrom, A, Bellafante, E, Jansen, E, Adorini, L, Shapiro, D, Jones, P, Marchesi, JR, Marschall, HU, Williamson, C. Obeticholic acid ameliorates dyslipidemia but not glucose tolerance in mouse model of gestational diabetes. Am J Physiol Endocrinol Metab. 2019. 317:E399-e410. doi:10.1152/ajpendo.00407.2018.

379. McKenzie, KM, Dissanayake, HU, McMullan, R, Caterson, ID, Celermajer, DS, Gordon, A, Hyett, J, Meroni, A, Phang, M, Raynes-Greenow, C, Polson, JW, Skilton, MR. Quantity and Quality of Carbohydrate Intake during Pregnancy, Newborn Body Fatness and Cardiac Autonomic Control: Conferred Cardiovascular Risk? Nutrients. 2017. 9. doi:10.3390/nu9121375.

380. Meinila, J, Koivusalo, SB, Valkama, A, Rono, K, Erkkola, M, Kautiainen, H, StachLempinen, B, Eriksson, JG. Nutrient intake of pregnant women at high risk of gestational diabetes. Food \& nutrition research. 2015. 59. doi:10.3402/fnr.v59.26676.

\section{Study Design; Outcome}

Intervention/Exposure

Outcome

\section{Outcome}

Intervention/Exposure

Study Design

Conference abstract

Intervention/Exposure; Human

Intervention/Exposure; Outcome 
381. Meinila, J, Valkama, A, Koivusalo, SB, Stach-Lempinen, B, Lindstrom, J, Kautiainen, $\mathrm{H}$,

Eriksson, JG, Erkkola, M. Healthy Food Intake Index (HFII) - Validity and reproducibility in a gestational-diabetes-risk population. BMC Public Health. 2016. 16:680. doi:10.1186/s12889-016-3303-7.

382. Meinila, J, Valkama, A, Koivusalo, SB, Stach-Lempinen, B, Rono, K, Lindstrom, J,

Population; Outcome

Kautiainen, H, Eriksson, JG, Erkkola, M. Is improvement in the Healthy Food Intake Index (HFII) related to a lower risk for gestational diabetes? Br J Nutr. 2017. 117:1103-1109. doi:10.1017/s0007114517001015.

383. Meltzer, HM, Brantsaeter, AL, Nilsen, RM, Magnus, P, Alexander, J, Haugen, M. Effect of

Study Design; Outcome dietary factors in pregnancy on risk of pregnancy complications: results from the Norwegian Mother and Child Cohort Study. Am J Clin Nutr. 2011. 94:1970s-1974s. doi:10.3945/ajcn.110.001248.

384. Meng, Y, Groth, SW, Li, D. The Association between Obesity-Risk Genes and Gestational Intervention/Exposure Weight Gain Is Modified by Dietary Intake in African American Women. J Nutr Metab. 2018. 2018:5080492. doi: $10.1155 / 2018 / 5080492$.

385. Meng, Y, Groth, SW, Stewart, P, Smith, JA. An Exploration of the Determinants of Gestational Weight Gain in African American Women: Genetic Factors and Energy Expenditure. Biol Res Nurs. 2018. 20:118-125. doi:10.1177/1099800417743326.

386. Merkx, A, Ausems, M, Bude, L, de Vries, R, Nieuwenhuijze, MJ. Weight gain in healthy pregnant women in relation to pre-pregnancy $\mathrm{BMI}$, diet and physical activity. Midwifery. 2015. 31:693-701. doi:10.1016/j.midw.2015.04.008.

387. Micali, N, Al Essimii, H, Field, AE, Treasure, J. Pregnancy loss of control over eating: A longitudinal study of maternal and child outcomes. American Journal of Clinical Nutrition. 2018. 108:101-107. doi:10.1093/ajcn/nqy040.

388. Mielke, RT. Determinants of excessive gestational weight gain in Mexican American women in Los Angeles. 2010. :174 p-174 p.

389. Mikkelsen, TB, Osler, M, Orozova-Bekkevold, I, Knudsen, VK, Olsen, SF. Association between fruit and vegetable consumption and birth weight: a prospective study among 43,585 Danish women. Scand J Public Health. 2006. 34:616-22. doi:10.1080/14034940600717688.

390. Milajerdi, A, Tehrani, H, Haghighatdoost, F, Larijani, B, Surkan, PJ, Azadbakht, L. Associations between higher egg consumption during pregnancy with lowered risks of high blood pressure and gestational diabetes mellitus. Int J Vitam Nutr Res. 2018. 88:166-175. doi:10.1024/0300-9831/a000505.

Intervention/Exposure

\section{Dissertation}

\section{Outcome}


391. Min, Y, Djahanbakhch, O, Hutchinson, J, Bhullar, AS, Raveendran, M, Hallot, A, Eram, S, Namugere, I, Nateghian, S, Ghebremeskel, K. Effect of docosahexaenoic acid-enriched fish oil supplementation in pregnant women with Type 2 diabetes on membrane fatty acids and fetal body composition--double-blinded randomized placebo-controlled trial. Diabet Med. 2014. 31:1331-40. doi:10.1111/dme.12524.

392. Mirmiran, P, Hosseinpour-Niazi, S, Moghaddam-Banaem, L, Lamyian, M, Goshtasebi, A, Azizi, F. Inverse relation between fruit and vegetable intake and the risk of gestational diabetes mellitus. Int J Vitam Nutr Res. 2019. :1-8. doi:10.1024/0300-9831/a000475.

393. Mise, N, Ohtsu, M, Ikegami, A, Mizuno, A, Cui, X, Kobayashi, Y, Nakagi, Y, Nohara, K, Yoshida, T, Kayama, F. Hijiki seaweed consumption elevates levels of inorganic arsenic intake in Japanese children and pregnant women. Food Addit Contam Part A Chem Anal Control Expo Risk Assess. 2019. 36:84-95. doi:10.1080/19440049.2018.1562228.

394. Mitchell, EA, Robinson, E, Clark, PM, Becroft, DM, Glavish, N, Pattison, NS, Pryor, JE, Thompson, JM, Wild, CJ. Maternal nutritional risk factors for small for gestational age babies in a developed country: a case-control study. Arch Dis Child Fetal Neonatal Ed. 2004. 89:F431-5. doi:10.1136/adc.2003.036970.

395. Miyake, Y, Okubo, H, Sasaki, S, Tanaka, K, Hirota, Y. Maternal dietary patterns during pregnancy and risk of wheeze and eczema in Japanese infants aged 16-24 months: the Osaka Maternal and Child Health Study. Pediatr Allergy Immunol. 2011. 22:734-41. doi:10.1111/j.1399-3038.2011.01176.x.

396. Moller, UK, Streym, S, Heickendorff, L, Mosekilde, L, Rejnmark, L. Effects of 25OHD concentrations on chances of pregnancy and pregnancy outcomes: a cohort study in healthy Danish women. Eur J Clin Nutr. 2012. 66:862-8. doi:10.1038/ejcn.2012.18.

397. Molyneaux, E, Poston, L, Khondoker, M, Howard, LM. Obesity, antenatal depression, diet Intervention/Exposure; Outcome and gestational weight gain in a population cohort study. Arch Womens Ment Health. 2016. 19:899-907. doi:10.1007/s00737-016-0635-3.

398. Monteagudo, C, Mariscal-Arcas, M, Heras-Gonzalez, L, Ibanez-Peinado, D, Rivas, A, Olea-Serrano, $F$. Effects of maternal diet and environmental exposure to organochlorine pesticides on newborn weight in Southern Spain. Chemosphere. 2016. 156:135-142. doi:10.1016/j.chemosphere.2016.04.103.

399. Moore, VM, Davies, MJ, Willson, KJ, Worsley, A, Robinson, JS. Dietary composition of pregnant women is related to size of the baby at birth. J Nutr. 2004. 134:1820-6. doi:10.1093/jn/134.7.1820.

400. Moradi, M, Maracy, MR, Esmaillzadeh, A, Surkan, PJ, Azadbakht, L. Associations Between Dietary Energy Density in Mothers and Growth of Breastfeeding Infants During the First 4 Months of Life. J Am Coll Nutr. 2018. :1-7. doi:10.1080/07315724.2018.1465486.

\section{Outcome}

Outcome

Intervention/Exposure; Outcome

Intervention/Exposure; Outcome

Intervention/Exposure

Intervention/Exposure 
401. Moran, LJ, Flynn, AC, Louise, J, Deussen, AR, Dodd, JM. The effect of a lifestyle

intervention on pregnancy and postpartum dietary patterns determined by factor analysis. Obesity (Silver Spring). 2017. 25:1022-1032. doi:10.1002/oby.21848.

402. Moran, LJ, McNaughton, SA, Sui, Z, Cramp, C, Deussen, AR, Grivell, RM, Dodd, JM. The characterisation of overweight and obese women who are under reporting energy intake during pregnancy. BMC Pregnancy Childbirth. 2018. 18:204. doi:10.1186/s12884-0181826-x.

403. Moran, LJ, Sui, Z, Cramp, CS, Dodd, JM. A decrease in diet quality occurs during pregnancy in overweight and obese women which is maintained post-partum. Int $\mathrm{J}$ Obes (Lond). 2013. 37:704-11. doi:10.1038/ijo.2012.129.

404. Moreno, MA. Advice for patients. Breastfeeding as obesity prevention. Arch Pediatr Adolesc Med. 2011. 165:772. doi:10.1001/archpediatrics.2011.140.

405. Moreno-Castilla, $C$, Hernandez, M, Bergua, M, Alvarez, MC, Arce, MA, Rodriguez, K, Martinez-Alonso, M, Iglesias, M, Mateu, M, Santos, MD, Pacheco, LR, Blasco, Y, Martin, E, Balsells, N, Aranda, N, Mauricio, D. Low-Carbohydrate diet for the treatment of gestational diabetes mellitus: A randomized controlled trial. Diabetes Care. 2013. 36:2233-2238. doi:10.2337/dc12-2714.

406. Morisset, AS, Cote, JA, Michaud, A, Robitaille, J, Tchernof, A, Dube, MC, Veillette, J, Weisnagel, SJ. Dietary intakes in the nutritional management of gestational diabetes mellitus. Can J Diet Pract Res. 2014. 75:64-71. doi:10.3148/75.2.2014.64.

407. Moses, RG, Casey, SA, Quinn, EG, Cleary, JM, Tapsell, LC, Milosavljevic, M, Petocz, P, Brand-Miller, JC. Pregnancy and Glycemic Index Outcomes study: Effects of low glycemic index compared with conventional dietary advice on selected pregnancy outcomes. American Journal of Clinical Nutrition. 2014. 99:517-523. doi:10.3945/ajcn.113.074138.

408. Moses, RG, Luebcke, M, Davis, WS, Coleman, KJ, Tapsell, LC, Petocz, P, Brand-Miller JC. Effect of a low-glycemic-index diet during pregnancy on obstetric outcomes. Am J Clin Nutr. 2006. 84:807-12. doi:10.1093/ajcn/84.4.807.

409. Most, J, Amant, MS, Hsia, DS, Altazan, AD, Thomas, DM, Gilmore, LA, Vallo, PM, Beyl, RA, Ravussin, E, Redman, LM. Evidence-based recommendations for energy intake in pregnant women with obesity. J Clin Invest. 2019. 130:4682-4690. doi:10.1172/jci130341.

410. Most, J, Rebello, CJ, Altazan, AD, Martin, CK, Amant, MS, Redman, LM. Behavioral Determinants of Objectively Assessed Diet Quality in Obese Pregnancy. Nutrients. 2019. 11. doi:10.3390/nu11071446.

411. Mujsindi, W, Habash, D, Childs, G. Impact of nutrition education on gestational weight gain in obese pregnant women. American journal of obstetrics and gynecology. 2014. 210:S188. doi:10.1016/j.ajog.2013.10.402.

\section{Study Design}

Health Status

\section{Comparator}

Intervention/Exposure

Intervention/Exposure

Intervention/Exposure

Outcome

Abstract 
412. Muliyil, DE, Rose, A, Senthamizh, SV, Chatterjee, T, Helan, J, Kang, G, Muliyil, J.

Prevalence and Risk Factors of Vitamin A Deficiency in Children and Women of

Childbearing Age in a Southern Indian Tribal Population: A Cross-Sectional Study. Indian J Community Med. 2019. 44:162-165. doi:10.4103/ijcm.IJCM_213_18.

413. Mullaney, L, O'Higgins, AC, Cawley, S, Kennedy, R, McCartney, D, Turner, MJ. Breastfeeding and postpartum maternal weight trajectories. Public Health Nutr. 2016. 19:1397404. doi:10.1017/s1368980015002967.

414. Munda, A, Starcic Erjavec, M, Molan, K, Ambrozic Avgustin, J, Zgur-Bertok, D, Pongrac Barlovic, D. Association between pre-pregnancy body weight and dietary pattern with large-for-gestational-age infants in gestational diabetes. Diabetol Metab Syndr. 2019. 11:68. doi:10.1186/s13098-019-0463-5.

415. Murrin, CM, Heinen, MM, Kelleher, CC. Are Dietary Patterns of Mothers during Pregnancy Outcome Related to Children's Weight Status? Evidence from the Lifeways Cross- Generational Cohort Study. AIMS Public Health. 2015. 2:274-296. doi:10.3934/publichealth.2015.3.274.

416. Navarro, $P$, Mehegan, J, Murrin, CM, Kelleher, CC, Phillips, CM. Adherence to the Healthy Eating Index-2015 across Generations Is Associated with Birth Outcomes and Weight Status at Age 5 in the Lifeways Cross-Generation Cohort Study. Nutrients. 2019. 11. doi:10.3390/nu11040928.

417. Ng, SK, Cameron, CM, Hills, AP, McClure, RJ, Scuffham, PA. Socioeconomic disparities in prepregnancy BMI and impact on maternal and neonatal outcomes and postpartum weight retention: the EFHL longitudinal birth cohort study. BMC Pregnancy Childbirth. 2014. 14:314. doi:10.1186/1471-2393-14-314.

418. Nicklas, JM, Zera, CA, Seely, EW. Predictors of very early postpartum weight loss in women with recent gestational diabetes mellitus. J Matern Fetal Neonatal Med. 2018. :17. doi:10.1080/14767058.2018.1487937.

419. Nikniaz, L, Jr, Mahdavi, R, Arefhoesseini, SR, Sowti Khiabani, M. Association between fat content of breast milk and maternal nutritional status and infants' weight in tabriz, iran. Malays J Nutr. 2009. 15:37-44.

420. Nikniaz, L, Mahavi, R, Ostadrahimi, A, Nikniaz, Z, Taghipour, S. Synbiotic supplementation is not effective on breast milk selenium concentrations and growth of exclusively breast fed infants: a pilot study. Int J Vitam Nutr Res. 2019. 1-7. doi:10.1024/0300-9831/a000549.

421. Nikniaz, L, Mahdavi, R, Gargari, BP, Gayem Magami, SJ, Nikniaz, Z. Maternal body mass index, dietary intake and socioeconomic status: differential effects on breast milk zinc, copper and iron content. Health Promot Perspect. 2011. 1:140-6. doi:10.5681/hpp.2011.015.

Population

Intervention/Exposure; Outcome

Outcome

Study Design; Intervention/Exposure

Intervention/Exposure

Intervention/Exposure; Outcome

Intervention/Exposure; Outcome

Study Design; Intervention/Exposure 
422. Normia, J, Niinivirta-Joutsa, K, Isolauri, E, Jääskeläinen, SK, Laitinen, K. Perinatal nutrition impacts on the functional development of the visual tract in infants. Pediatric Research. 2019. 85:72-78. doi:10.1038/s41390-018-0161-2.

423. Northstone, $\mathrm{K}$, Emmett, $\mathrm{P}$, Rogers, I. Dietary patterns in pregnancy and associations with

Outcome socio-demographic and lifestyle factors. Eur J Clin Nutr. 2008. 62:471-9. doi:10.1038/sj.ejcn.1602741.

424. Nunes, MA, Ferri, CP, Manzolli, $P$, Soares, RM, Drehmer, M, Buss, C, Giacomello, A, Hoffmann, JF, Ozcariz, S, Melere, C, Manenti, CN, Camey, S, Duncan, BB, Schmidt, MI. Nutrition, mental health and violence: from pregnancy to postpartum Cohort of women attending primary care units in Southern Brazil--ECCAGE study. BMC Psychiatry. 2010. 10:66. doi:10.1186/1471-244x-10-66.

425. Nunes, MA, Pinheiro, AP, Camey, SA, Schmidt, MI. Binge eating during pregnancy and birth outcomes: a cohort study in a disadvantaged population in Brazil. Int $\mathrm{J}$ Eat Disord. 2012. 45:827-31. doi:10.1002/eat.22024.

426. Nykjaer, C, Higgs, C, Greenwood, DC, Simpson, NAB, Cade, JE, Alwan, NA. Maternal Fatty Fish Intake Prior to and during Pregnancy and Risks of Adverse Birth Outcomes: Findings from a British Cohort. Nutrients. 2019. 11. doi:10.3390/nu11030643.

427. O'Brien, CM, Louise, J, Deussen, A, Dodd, JM. In Overweight or Obese Pregnant Women, Maternal Dietary Factors are not Associated with Fetal Growth and Adiposity. Nutrients. 2018. 10. doi:10.3390/nu10070870.

428. O'Brien, EC, Alberdi, G, Geraghty, AA, McAuliffe, FM. Lower education predicts poor response to dietary intervention in pregnancy, regardless of neighbourhood affluence: secondary analysis from the ROLO randomised control trial. Public Health Nutr. 2017. 20:2959-2969. doi:10.1017/s1368980017001951.

429. O'Brien, EC, Geraghty, AA, O'Sullivan, EJ, Riordan, JA, Horan, MK, Larkin, E, Donnelly, J, Mehegan, J, Twomey, PJ, McAuliffe, FM. Five-year follow up of a low glycaemic index dietary randomised controlled trial in pregnancy-no long-term maternal effects of a dietary intervention. Bjog. 2019. 126:514-524. doi:10.1111/1471-0528.15500.

430. Oken, E, Ning, Y, Rifas-Shiman, SL, Rich-Edwards, JW, Olsen, SF, Gillman, MW. Diet during pregnancy and risk of preeclampsia or gestational hypertension. Ann Epidemiol. 2007. 17:663-8. doi:10.1016/j.annepidem.2007.03.003.

431. Olafsdottir, AS, Magnusardottir, AR, Thorgeirsdottir, H, Hauksson, A, Skuladottir, GV, Steingrimsdottir, L. Relationship between dietary intake of cod liver oil in early pregnancy and birthweight. Bjog. 2005. 112:424-9. doi:10.1111/j.1471-0528.2005.00477.x.

432. Olafsdottir, AS, Skuladottir, GV, Thorsdottir, I, Hauksson, A, Steingrimsdottir, L. Combined effects of maternal smoking status and dietary intake related to weight gain and birth size parameters. Bjog. 2006. 113:1296-302. doi:10.1111/j.1471-0528.2006.01077.x. 
433. Olafsdottir, AS, Skuladottir, GV, Thorsdottir, I, Hauksson, A, Steingrimsdottir, L. Maternal diet in early and late pregnancy in relation to weight gain. Int J Obes (Lond). 2006. 30:492-9. doi:10.1038/sj.ijo.0803184.

434. Olmedo-Requena, R, Gomez-Fernandez, J, Amezcua-Prieto, C, Mozas-Moreno, J, Khan, Study Design KS, Jimenez-Moleon, JJ. Pre-Pregnancy Adherence to the Mediterranean Diet and Gestational Diabetes Mellitus: A Case-Control Study. Nutrients. 2019. 11. doi:10.3390/nu11051003.

435. Olson, CM, Strawderman, MS, Hinton, PS, Pearson, TA. Gestational weight gain and postpartum behaviors associated with weight change from early pregnancy to $1 \mathrm{y}$ postpartum. Int J Obes Relat Metab Disord. 2003. 27:117-27. doi:10.1038/sj.ijo.0802156.

436. Olson, CM, Strawderman, MS. Modifiable behavioral factors in a biopsychosocial model predict inadequate and excessive gestational weight gain. J Am Diet Assoc. 2003. 103:48-54. doi:10.1053/jada.2003.50001.

437. Opie, RS, Neff, M, Tierney, AC. A behavioural nutrition intervention for obese pregnant women: Effects on diet quality, weight gain and the incidence of gestational diabetes. Aust N Z J Obstet Gynaecol. 2016. 56:364-73. doi:10.1111/ajo.12474.

438. Orloff, NC, Flammer, A, Hartnett, J, Liquorman, S, Samelson, R, Hormes, JM. Food cravings in pregnancy: Preliminary evidence for a role in excess gestational weight gain. Appetite. 2016. 105:259-65. doi:10.1016/j.appet.2016.04.040.

439. Ostadrahimi, A, Nikniaz, L, Mahdavi, R, Hejazi, MA, Nikniaz, Z. Effects of synbiotic supplementation on lactating mothers' energy intake and BMI, and infants' growth. Int $\mathrm{J}$ Food Sci Nutr. 2013. 64:711-4. doi:10.3109/09637486.2013.775229.

440. Ostbye, T, Peterson, BL, Krause, KM, Swamy, GK, Lovelady, CA. Predictors of postpartum weight change among overweight and obese women: results from the Active Mothers Postpartum study. J Womens Health (Larchmt). 2012. 21:215-22. doi:10.1089/jwh.2011.2947.

441. Overby, NC, Hillesund, ER, Sagedal, LR, Vistad, I, Bere, E. The Fit for Delivery study: rationale for the recommendations and test-retest reliability of a dietary score measuring adherence to 10 specific recommendations for prevention of excessive weight gain during pregnancy. Matern Child Nutr. 2015. 11:20-32. doi:10.1111/mcn.12026.

442. Padilha, P, Felizardo, C, Saunders, C, Cunha, L, Pinheiro, A, Belfort, G, Santos, K, Ferreira, N. Consumption of Ultraprocessed Foods by Pregnant Women with Diabetes Mellitus (P11-021-19). Curr Dev Nutr. 2019. 3. doi:10.1093/cdn/nzz048.P11-021-19.

443. Pajaujiene, S, Dabasinskiene, L, Santos-Rocha, R. Health promotion program for improving women's body composition and active lifestyle in postpartum: A pilot study. Acta Medica Mediterranea. 2018. 34:1365-1375. doi:10.19193/0393-6384_2018_5_209.

Intervention/Exposure

Intervention/Exposure

Intervention/Exposure

Study Design; Intervention/Exposure

Intervention/Exposure; Comparator

Intervention/Exposure; Population

Intervention/Exposure; Outcome Abstract Intervention/Exposure 
444. Paknahad, Z, Fallah, A, Moravejolahkami, AR. Maternal Dietary Patterns and Their Association with Pregnancy Outcomes. Clin Nutr Res. 2019. 8:64-73. doi:10.7762/cnr.2019.8.1.64.

445. Palmer, JR, Kipping-Ruane, K, Wise, LA, Yu, J, Rosenberg, L. Lactation in Relation to Long-Term Maternal Weight Gain in African-American Women. Am J Epidemiol. 2015. 181:932-9. doi:10.1093/aje/kwv027.

446. Papadopoulou, E, Kogevinas, M, Botsivali, M, Pedersen, M, Besselink, H, Mendez, MA, Fleming, S, Hardie, LJ, Knudsen, LE, Wright, J, Agramunt, S, Sunyer, J, Granum, B, Gutzkow, KB, Brunborg, G, Alexander, J, Meltzer, HM, Brantsaeter, AL, Sarri, K, Chatzi, L, Merlo, DF, Kleinjans, JC, Haugen, M. Maternal diet, prenatal exposure to dioxin-like compounds and birth outcomes in a European prospective mother-child study (NewGeneris). Sci Total Environ. 2014. 484:121-8. doi:10.1016/j.scitotenv.2014.03.047.

447. Parisi, F, Rousian, M, Huijgen, NA, Koning, AHJ, Willemsen, SP, de Vries, JHM, Cetin, I, Steegers, EAP, Steegers-Theunissen, RPM. Periconceptional maternal 'high fish and olive oil, low meat' dietary pattern is associated with increased embryonic growth: The Rotterdam Periconceptional Cohort (Predict) Study. Ultrasound Obstet Gynecol. 2017. 50:709-716. doi:10.1002/uog.17408.

448. Park, HJ, Lee, J, Kim, JM, Lee, HA, Kim, SH, Kim, Y. A study of snack consumption, night-eating habits, and nutrient intake in gestational diabetes mellitus. Clin Nutr Res. 2013. 2:42-51. doi:10.7762/cnr.2013.2.1.42.

449. Parker, H, McCurdy, K, Tovar, A, Vadiveloo, M. The Relationship Between Gestational Weight Gain, Pre-pregnancy BMI, and Prenatal Diet Quality (P18-039-19). Curr Dev Nutr. 2019. 3. doi:10.1093/cdn/nzz039.P18-039-19.

450. Parker, HW, Tovar, A, McCurdy, K, Vadiveloo, M. Associations between pre-pregnancy $\mathrm{BMI}$, gestational weight gain, and prenatal diet quality in a national sample. PLoS One. 2019. 14:e0224034. doi:10.1371/journal.pone.0224034.

451. Parlapani, E, Agakidis, C, Karagiozoglou-Lampoudi, T, Sarafidis, K, Agakidou, E, Athanasiadis, A, Diamanti, E. The Mediterranean diet adherence by pregnant women delivering prematurely: association with size at birth and complications of prematurity. $J$ Matern Fetal Neonatal Med. 2017. :1-8. doi:10.1080/14767058.2017.1399120.

452. Pathirathna, ML, Sekijima, K, Sadakata, M, Fujiwara, N, Muramatsu, Y, Wimalasiri, KMS. Impact of Second Trimester Maternal Dietary Intake on Gestational Weight Gain and Neonatal Birth Weight. Nutrients. 2017. 9. doi:10.3390/nu9060627.

453. Patwardhan, G, Soni, A, Rachwani, N, Kadam, S, Patole, S, Pandit, A. Factors Associated with Time to Full Feeds in Preterm Very Low Birth Weight Infants. J Trop Pediatr. 2018. 64:495-500. doi:10.1093/tropej/fmx102. 
454. Paul, IM, Williams, JS, Anzman-Frasca, S, Beiler, JS, Makova, KD, Marini, ME, Hess, LB, Rzucidlo, SE, Verdiglione, N, Mindell, JA, Birch, LL. The Intervention Nurses Start Infants Growing on Healthy Trajectories (INSIGHT) study. BMC Pediatr. 2014. 14:184. doi:10.1186/1471-2431-14-184.

455. Pauley, AM, Hohman, E, Savage, JS, Rivera, DE, Guo, P, Leonard, KS, Symons Downs, D. Gestational Weight Gain Intervention Impacts Determinants of Healthy Eating and Exercise in Overweight/Obese Pregnant Women. Journal of Obesity. 2018. 2018. doi:10.1155/2018/6469170.

456. Peccei, A, Blake-Lamb, T, Rahilly, D, Hatoum, I, Bryant, A. Intensive Prenatal Nutrition Counseling in a Community Health Setting. Obstetrics and Gynecology. 2017. 130:423432. doi:10.1097/AOG.0000000000002134.

457. Pellonpera, O, Koivuniemi, E, Vahlberg, T, Mokkala, K, Tertti, K, Ronnemaa, T, Laitinen, $\mathrm{K}$. Dietary quality influences body composition in overweight and obese pregnant women. Clin Nutr. 2019. 38:1613-1619. doi:10.1016/j.clnu.2018.08.029.

458. Peraita-Costa, I, Llopis-Gonzalez, A, Perales-Marin, A, Sanz, F, Llopis-Morales, A, Morales-Suarez-Varela, M. A Retrospective Cross-Sectional Population-Based Study on Prenatal Levels of Adherence to the Mediterranean Diet: Maternal Profile and Effects on the Newborn. Int J Environ Res Public Health. 2018. 15. doi:10.3390/ijerph15071530.

459. Perez-Ferre, N, Del Valle, L, Torrejon, MJ, Barca, I, Calvo, MI, Matia, P, Rubio, MA, CallePascual, AL. Diabetes mellitus and abnormal glucose tolerance development after gestational diabetes: A three-year, prospective, randomized, clinical-based, Mediterranean lifestyle interventional study with parallel groups. Clin Nutr. 2015. 34:579-85. doi:10.1016/j.clnu.2014.09.005.

460. Petersen, SB, Rasmussen, MA, Olsen, SF, Vestergaard, P, Mølgaard, C, Halldorsson, T, Strøm, M. Maternal Dietary Patterns during Pregnancy in Relation to Offspring Forearm Fractures: Prospective Study from the Danish National Birth Cohort. Nutrients. 2015. 7:2382-2400. doi:10.3390/nu7042382.

461. Petrella, E, Bruno, R, Pedrielli, G, Bertarini, V, Neri, I, Facchinetti, F. A customized low glycaemic-index (GI) diet prevents both the gestational diabetes mellitus (GDM) and the large for gestational age (LGA) babies in overweight/obese pregnant women. American journal of obstetrics and gynecology. 2016. 214:S159-

462. Petrella, E, Facchinetti, F, Bertarini, V, Pignatti, L, Neri, I, Battistini, NC. Occurrence of pregnancy complications in women with $\mathrm{BMI}>25$ submitted to a healthy lifestyle and eating habits program. American journal of obstetrics and gynecology. 2013. 208:S33S34. doi:10.1016/j.ajog.2012.10.229.

\section{Intervention/Exposure; Outcome}

Intervention/Exposure; Comparator

Intervention/Exposure

Outcome; Outcome not reported by DP adherence

Study Design

Population; Outcome

\section{Outcome}

Abstract

Abstract 
463. Petrella, E, Malavolti, M, Bertarini, V, Pignatti, L, Neri, I, Battistini, NC, Facchinetti, F. Gestational weight gain in overweight and obese women enrolled in a healthy lifestyle and eating habits program. J Matern Fetal Neonatal Med. 2014. 27:1348-52. doi:10.3109/14767058.2013.858318.

464. Phang, M, Dissanayake, HU, McMullan, RL, Hyett, J, Gordon, A, Garg, ML, Skilton, MR. Increased alpha-Linolenic Acid Intake during Pregnancy is Associated with Higher Offspring Birth Weight. Curr Dev Nutr. 2019. 3:nzy081. doi:10.1093/cdn/nzy081.

465. Phelan, S, Phipps, MG, Abrams, B, Darroch, F, Grantham, K, Schaffner, A, Wing, RR. Does behavioral intervention in pregnancy reduce postpartum weight retention? Twelvemonth outcomes of the Fit for Delivery randomized trial. Am J Clin Nutr. 2014. 99:302-11. doi:10.3945/ajen.113.070151.

466. Phelan, S, Phipps, MG, Abrams, B, Darroch, F, Schaffner, A, Wing, RR. Randomized trial of a behavioral intervention to prevent excessive gestational weight gain: the fit for delivery study. Obstetrical \& gynecological survey. 2011. 66:471-472. doi:10.1097/OGX.0b013e31823520b3.

467. Phelan, S, Wing, RR, Brannen, A, McHugh, A, Hagobian, TA, Schaffner, A, Jelalian, E, Hart, CN, Scholl, TO, Munoz-Christian, K, Yin, E, Phipps, MG, Keadle, S, Abrams, B. Randomized controlled clinical trial of behavioral lifestyle intervention with partial meal replacement to reduce excessive gestational weight gain. American Journal of Clinical Nutrition. 2018. 107:183-194. doi:10.1093/ajcn/nqx043.

468. Pinto, TJ, Farias, DR, Rebelo, F, Lepsch, J, Vaz, JS, Moreira, JD, Cunha, GM, Kac, G. Lower inter-partum interval and unhealthy life-style factors are inversely associated with $n$ 3 essential fatty acids changes during pregnancy: a prospective cohort with Brazilian women. PLoS One. 2015. 10:e0121151. doi:10.1371/journal.pone.0121151.

469. Plante, AS, Savard, C, Lemieux, S, Carbonneau, E, Robitaille, J, Provencher, V, Morisset, AS. Trimester-Specific Intuitive Eating in Association With Gestational Weight Gain and Diet Quality. J Nutr Educ Behav. 2019. 51:677-683. doi:10.1016/j.jneb.2019.01.011.

470. Pollak, KI, Alexander, SC, Bennett, G, Lyna, P, Coffman, CJ, Bilheimer, A, Farrell, D, Bodner, ME, Swamy, GK, Ostbye, T. Weight-related SMS texts promoting appropriate pregnancy weight gain: a pilot study. Patient education and counseling. 2014. 97:256-260. doi:10.1016/j.pec.2014.07.030.

471. Pomerleau, CS, Brouwer, RJ, Jones, LT. Weight concerns in women smokers during pregnancy and postpartum. Addict Behav. 2000. 25:759-67.

Intervention/Exposure; Outcome

Intervention/Exposure; Population

Editorial comment

Intervention/Exposure

Intervention/Exposure; Outcome

Intervention/Exposure; Outcome

Intervention/Exposure

Intervention/Exposure 
472. Poston, L, Bell, R, Croker, H, Flynn, AC, Godfrey, KM, Goff, L, Hayes, L, Khazaezadeh, N, Nelson, SM, Oteng-Ntim, E, Pasupathy, D, Patel, N, Robson, SC, Sandall, J, Sanders, TA,

Sattar, N, Seed, PT, Wardle, J, Whitworth, MK, Briley, AL. Effect of a behavioural intervention in obese pregnant women (the UPBEAT study): a multicentre, randomised controlled trial. Lancet Diabetes Endocrinol. 2015. 3:767-77. doi:10.1016/s22138587(15)00227-2.

473. Pullmer, R, Zaitsoff, S, Cobb, R. Body Satisfaction During Pregnancy: The Role of HealthRelated Habit Strength. Matern Child Health J. 2018. 22:391-400. doi:10.1007/s10995017-2406-9.

474. Quick, V, Martin-Biggers, J, Byrd-Bredbenner, C. Moms' Eating, Sleeping, and Physical Activity Behaviors Differ By Weight Status: Implications for Nutrition Education Interventions. Journal of the Academy of Nutrition \& Dietetics. 2016. 116:A24-A24. doi:10.1016/j.jand.2016.06.077.

475. Quinlivan, JA, Lam, LT, Fisher, J. A randomised trial of a four-step multidisciplinary approach to the antenatal care of obese pregnant women. Aust N Z J Obstet Gynaecol. 2011. 51:141-6. doi:10.1111/j.1479-828X.2010.01268.x.

476. Quinn, EA, Kuzawa, CW. A dose-response relationship between fish consumption and human milk DHA content among Filipino women in Cebu City, Philippines. Acta Paediatr. 2012. 101:e439-45. doi:10.1111/j.1651-2227.2012.02777.x.

477. Quinn, EA, Largado, F, Power, M, Kuzawa, CW. Predictors of breast milk macronutrient composition in Filipino mothers. Am J Hum Biol. 2012. 24:533-40. doi:10.1002/ajhb.22266.

478. Radesky, JS, Oken, E, Rifas-Shiman, SL, Kleinman, KP, Rich-Edwards, JW, Gillman, MW. Diet during early pregnancy and development of gestational diabetes. Paediatr Perinat Epidemiol. 2008. 22:47-59. doi:10.1111/j.1365-3016.2007.00899.x.

479. Radwan, $H$, Hashim, $M$, Shaker Obaid, R, Hasan, H, Naja, F, Al Ghazal, $H$, Jan Jan Mohamed, H, Rizk, R, Al Hilali, M, Rayess, R, Izzaldin, G. The Mother-Infant Study Cohort (MISC): Methodology, challenges, and baseline characteristics. PLoS One. 2018. 13:e0198278. doi:10.1371/journal.pone.0198278.

480. Ramage, SM, McCargar, LJ, Berglund, C, Harber, V, Bell, RC. Assessment of PrePregnancy Dietary Intake with a Food Frequency Questionnaire in Alberta Women. Nutrients. 2015. 7:6155-6166. doi:10.3390/nu7085277.

481. Ramon, R, Ballester, F, Iniguez, C, Rebagliato, M, Murcia, M, Esplugues, A, Marco, A, Garcia de la Hera, M, Vioque, J. Vegetable but not fruit intake during pregnancy is associated with newborn anthropometric measures. J Nutr. 2009. 139:561-7. doi:10.3945/jn.108.095596. 
482. Rauh, K, Gunther, J, Kunath, J, Stecher, L, Hauner, H. Lifestyle intervention to prevent excessive maternal weight gain: mother and infant follow-up at 12 months postpartum. BMC pregnancy and childbirth. 2015. 15. doi:10.1186/s12884-015-0701-2.

483. Redman, LM, Gilmore, LA, Breaux, J, Thomas, DM, Elkind-Hirsch, K, Stewart, T, Hsia, DS, Burton, J, Apolzan, JW, Cain, LE, Altazan, AD, Ragusa, S, Brady, H, Davis, A, Tilford, JM, Sutton, EF, Martin, CK. Effectiveness of SmartMoms, a Novel eHealth Intervention for Management of Gestational Weight Gain: Randomized Controlled Pilot Trial. JMIR Mhealth Uhealth. 2017. 5:e133. doi:10.2196/mhealth.8228.

484. Renault, KM, Carlsen, EM, Norgaard, K, Nilas, L, Pryds, O, Secher, NJ, Cortes, D, Jensen, JE, Olsen, SF, Halldorsson, TI. Intake of carbohydrates during pregnancy in obese women is associated with fat mass in the newborn offspring. Am J Clin Nutr. 2015. 102:1475-81. doi:10.3945/ajcn.115.110551.

485. Renault, KM, Carlsen, EM, Norgaard, K, Nilas, L, Pryds, O, Secher, NJ, Olsen, SF, Halldorsson, TI. Intake of Sweets, Snacks and Soft Drinks Predicts Weight Gain in Obese Pregnant Women: Detailed Analysis of the Results of a Randomised Controlled Trial. PLoS One. 2015. 10:e0133041. doi:10.1371/journal.pone.0133041.

486. Renault, KM, Norgaard, K, Nilas, L, Carlsen, EM, Cortes, D, Pryds, O, Secher, NJ. The Treatment of Obese Pregnant Women (TOP) study: a randomized controlled trial of the effect of physical activity intervention assessed by pedometer with or without dietary intervention in obese pregnant women. Am J Obstet Gynecol. 2014. 210:134.e1-9. doi:10.1016/j.ajog.2013.09.029.

487. Renzaho, AM, Skouteris, H, Oldroyd, J. Preventing gestational diabetes mellitus among migrant women and reducing obesity and type 2 diabetes in their offspring: a call for culturally competent lifestyle interventions in pregnancy. J Am Diet Assoc. 2010. 110:1814-7. doi:10.1016/j.jada.2010.09.017.

488. Rerkasem, K, Wongthanee, A, Rerkasem, A, Chiowanich, P, Sritara, $P$, Pruenglampoo, S, Mangklabruks, A. Intrauterine nutrition and carotid intimal media thickness in young Thai adults. Asia Pac J Clin Nutr. 2012. 21:247-52.

489. Reyes, L, Garcia, R, Ruiz, S, Dehghan, M, Lopez-Jaramillo, P. Nutritional status among women with pre-eclampsia and healthy pregnant and non-pregnant women in a Latin American country. J Obstet Gynaecol Res. 2012. 38:498-504. doi:10.1111/j.14470756.2011.01763.x.

490. Rhodes, ET, Pawlak, DB, Takoudes, TC, Ebbeling, CB, Feldman, HA, Lovesky, MM, Cooke, EA, Leidig, MM, Ludwig, DS. Effects of a low-glycemic load diet in overweight and obese pregnant women: a pilot randomized controlled trial. Am J Clin Nutr. 2010. 92:1306-15. doi:10.3945/ajcn.2010.30130.

Intervention/Exposure

Intervention/Exposure; Outcome

Intervention/Exposure

Intervention/Exposure

\section{Study Design}

\section{Outcome}

Study Design; Outcome

Intervention/Exposure 
491. Rich-Edwards, JW, Stuart, JJ, Skurnik, G, Roche, AT, Tsigas, E, Fitzmaurice, GM, Wilkins-Haug, LE, Levkoff, SE, Seely, EW. Randomized Trial to Reduce Cardiovascular Risk in Women with Recent Preeclampsia. J Womens Health (Larchmt). 2019. doi:10.1089/jwh.2018.7523.

492. Rodriguez-Bernal, CL, Rebagliato, M, Iniguez, C, Vioque, J, Navarrete-Munoz, EM, Murcia, M, Bolumar, F, Marco, A, Ballester, F. Diet quality in early pregnancy and its effects on fetal growth outcomes: the Infancia y Medio Ambiente (Childhood and Environment) Mother and Child Cohort Study in Spain. Am J Clin Nutr. 2010. 91:1659-66. doi:10.3945/ajcn.2009.28866.

493. Rogers, I, Emmett, $P$, Ness, A, Golding, J. Maternal fish intake in late pregnancy and the frequency of low birth weight and intrauterine growth retardation in a cohort of British infants. J Epidemiol Community Health. 2004. 58:486-92. doi:10.1136/jech.2003.013565.

494. Rohatgi, KW, Tinius, RA, Cade, WT, Steele, EM, Cahill, AG, Parra, DC. Relationships between consumption of ultra-processed foods, gestational weight gain and neonatal outcomes in a sample of US pregnant women. PeerJ. 2017. 5:e4091. doi:10.7717/peerj.4091.

495. Romon, M, Nuttens, MC, Vambergue, A, Verier-Mine, O, Biausque, S, Lemaire, C, Fontaine, P, Salomez, JL, Beuscart, R. Higher carbohydrate intake is associated with decreased incidence of newborn macrosomia in women with gestational diabetes. J Am Diet Assoc. 2001. 101:897-902. doi:10.1016/s0002-8223(01)00220-6.

496. Ruiz-Gracia, T, Duran, A, Fuentes, M, Rubio, MA, Runkle, I, Carrera, EF, Torrejon, MJ, Bordiu, E, Valle, LD, Garcia de la Torre, N, Bedia, AR, Montanez, C, Familiar, C, CallePascual, AL. Lifestyle patterns in early pregnancy linked to gestational diabetes mellitus diagnoses when using IADPSG criteria. The St Carlos gestational study. Clin Nutr. 2016. 35:699-705. doi:10.1016/j.clnu.2015.04.017.

497. Sally, EOF, Anjos, LAD, Ramos, EG, Fonseca, VM, Silva, Bamd, Wahrlich, V. Dietary intake of pregnant adolescents cared for in primary health care units of a Brazilian urban municipality. Nutr Hosp. 2018. 35:596-605. doi:10.20960/nh.1412.

498. Salmenhaara, M, Uusitalo, L, Uusitalo, U, Kronberg-Kippila, C, Sinkko, H, Ahonen, S, Veijola, R, Knip, M, Kaila, M, Virtanen, SM. Diet and weight gain characteristics of pregnant women with gestational diabetes. Eur J Clin Nutr. 2010. 64:1433-40. doi:10.1038/ejcn.2010.167.

499. Salunkhe, AH, Pratinidhi, A, Salunkhe, JA, Kakade, SV, Mohite, VR, Hiremath, P. Frequency and nutrient content of meals of the mothers and the birth weight and gestational age of the baby. Journal of Krishna Institute of Medical Sciences University. 2018. 7:33-41. 
500. Samur, G, Akkus, O O, Ede, G, Ayaz, A, Akyol, A, Akkus, M, Danisman, N. Nutritional status among women with preeclampsia and healthy pregnant women. Progress in Nutrition. 2016. 18:360-368.

501. Santos, KD, Moreira, TM, Belfort, GP, Silva, Cfmd, Padilha, PC, Barros, DC, Saunders, C. Outcome Adaptation of DASH diet (Dietary Approach to Stop Hypertension) for postpartum nutritional care at primary healthcare. Rev Bras Epidemiol. 2019. 22:e190035. doi:10.1590/1980-549720190035.

502. Sartorelli, DS, Barbieri, $P$, Perdona, GC. Fried food intake estimated by the multiple source method is associated with gestational weight gain. Nutr Res. 2014. 34:667-73. doi:10.1016/j.nutres.2014.07.008.

503. Sauder, KA, Starling, AP, Shapiro, AL, Kaar, JL, Ringham, BM, Glueck, DH, Leiferman, JA, Siega-Riz, AM, Dabelea, D. Diet, physical activity and mental health status are associated with dysglycaemia in pregnancy: the Healthy Start Study. Diabet Med. 2016. 33:663-7. doi:10.1111/dme.13093.

504. Saunders, L, Guldner, L, Costet, N, Kadhel, P, Rouget, F, Monfort, C, Thome, JP, Multigner, L, Cordier, S. Effect of a Mediterranean diet during pregnancy on fetal growth and preterm delivery: results from a French Caribbean Mother-Child Cohort Study (TIMOUN). Paediatr Perinat Epidemiol. 2014. 28:235-44. doi:10.1111/ppe.12113.

505. Savard, C, Lemieux, S, Carbonneau, E, Provencher, V, Gagnon, C, Robitaille, J, Morisset, Outcome AS. Trimester-Specific Assessment of Diet Quality in a Sample of Canadian Pregnant Women. Int J Environ Res Public Health. 2019. 16. doi:10.3390/ijerph16030311.

506. Schoenaker, DA, Soedamah-Muthu, SS, Callaway, LK, Mishra, GD. Pre-pregnancy dietary patterns and risk of gestational diabetes mellitus: results from an Australian population-based prospective cohort study. Diabetologia. 2015. 58:2726-35. doi:10.1007/s00125-015-3742-1.

507. Schoenaker, DA, Soedamah-Muthu, SS, Mishra, GD. Quantifying the mediating effect of body mass index on the relation between a Mediterranean diet and development of maternal pregnancy complications: the Australian Longitudinal Study on Women's Health. Am J Clin Nutr. 2016. 104:638-45. doi:10.3945/ajcn.116.133884.

508. Sedaghat, F, Akhoondan, M, Ehteshami, M, Aghamohammadi, V, Ghanei, N, Mirmiran, P, Rashidkhani, B. Maternal Dietary Patterns and Gestational Diabetes Risk: A Case-Control Study. Journal of Diabetes Research. 2017. 2017. doi:10.1155/2017/5173926.

509. Senol Eren, N, Sencan, I, Aksoy, H, Koc, EM, Kasim, I, Kahveci, R, Samur, G, Ozkara, A. Evaluation of dietary habits during pregnancy. Turk J Obstet Gynecol. 2015. 12:89-95. doi:10.4274/tjod.79923.

Intervention/Exposure

Intervention/Exposure; Outcome

Study Design; Diet assessed after delivery

Population; Outcome

\section{Outcome}

\section{Outcome}

Study Design; Intervention/Exposure 
510. Shah, BS, Freeland-Graves, JH, Cahill, JM, Lu, H, Graves, GR. Diet quality as measured by the healthy eating index and the association with lipid profile in low-income women in early postpartum. J Am Diet Assoc. 2010. 110:274-9. doi:10.1016/j.jada.2009.10.038.

511. Shankar, K, Zhong, $Y$, Kang, $P$, Chintapalli, S, Thakali, K, Piccolo, $B$, Andres, A. Maternal BMI Modulates Placental Expression of Lipid Transport, Metabolism and Innate Immune Abstract Response Genes at Term (OR35-01-19). Curr Dev Nutr. 2019. 3. doi:10.1093/cdn/nzz048.OR35-01-19.

512. Shapiro, AL, Kaar, JL, Crume, TL, Starling, AP, Siega-Riz, AM, Ringham, BM, Glueck, DH Outcome Norris, JM, Barbour, LA, Friedman, JE, Dabelea, D. Maternal diet quality in pregnancy and neonatal adiposity: the Healthy Start Study. Int J Obes (Lond). 2016. 40:1056-62. doi:10.1038/ijo.2016.79.

513. Sharma, SS, Greenwood, DC, Simpson, NAB, Cade, JE. Is dietary macronutrient composition during pregnancy associated with offspring birth weight? An observational study. Br J Nutr. 2018. 119:330-339. doi:10.1017/s0007114517003609.

514. Sharma, SV, Chuang, RJ, Byrd-Williams, C, Danho, $M$, Upadhyaya, $M$, Berens, $P$, Hoelscher, DM. Pilot evaluation of HEAL - A natural experiment to promote obesity prevention behaviors among low-income pregnant women. Prev Med Rep. 2018. 10:254262. doi:10.1016/j.pmedr.2018.04.005.

515. Shieh, C, Yang, Z, Haas, DM, Carpenter, JS. Feasibility and Potential Benefits of a SelfMonitoring Enhanced Lifestyle Intervention to Prevent Excessive Gestational Weight Gain in Women Who Are Overweight or Obese. J Obstet Gynecol Neonatal Nurs. 2017. 46:182-196. doi:10.1016/j.jogn.2016.09.006.

516. Shiell, AW, Campbell-Brown, M, Haselden, S, Robinson, S, Godfrey, KM, Barker, DJ. High-meat, low-carbohydrate diet in pregnancy: relation to adult blood pressure in the offspring. Hypertension. 2001. 38:1282-8.

517. Shyam, S, Fatimah, A, Rohana, AG, Norasyikin, AW, Nik Shanita, S, Chinna, K, Mohd. Yusof, BN, Nor Azmi, K. Effect of Including Glycaemic Index (GI) Nutrition Education, within the Conventional Healthy Dietary Recommendation Framework, on Body Weight and Composition of Women with Prior Gestational Diabetes Mellitus: Results from a OneYear Randomised Controlled Trial. Malaysian Journal of Nutrition. 2015. 21:269-283.

518. Siegmund, T, Rad, NT, Ritterath, C, Siebert, G, Henrich, W, Buhling, KJ. Longitudinal changes in the continuous glucose profile measured by the CGMS in healthy pregnant women and determination of cut-off values. Eur J Obstet Gynecol Reprod Biol. 2008. 139:46-52. doi:10.1016/j.ejogrb.2007.12.006. 


\section{Citation}

519. Simmons, D, Devlieger, R, van Assche, A, Jans, G, Galjaard, S, Corcoy, R, Adelantado, JM, Dunne, F, Desoye, G, Harreiter, J, Kautzky-Willer, A, Damm, P, Mathiesen, ER, Jensen, DM, Andersen, L, Lapolla, A, Dalfra, MG, Bertolotto, A, Wender-Ozegowska, E, Zawiejska, A, Hill, D, Snoek, FJ, Jelsma, JG, van Poppel, MN. Effect of Physical Activity and/or Healthy Eating on GDM Risk: The DALI Lifestyle Study. J Clin Endocrinol Metab. 2017. 102:903-913. doi:10.1210/jc.2016-3455.

520. Simmons, D, Jelsma, JG, Galjaard, S, Devlieger, R, van Assche, A, Jans, G, Corcoy, R, Adelantado, JM, Dunne, F, Desoye, G, Harreiter, J, Kautzky-Willer, A, Damm, P, Mathiesen, ER, Jensen, DM, Andersen, LL, Lapolla, A, Dalfra, M, Bertolotto, A, WenderOzegowska, E, Zawiejska, A, Hill, D, Rebollo, P, Snoek, FJ, van Poppel, MN. Results From a European Multicenter Randomized Trial of Physical Activity and/or Healthy Eating to Reduce the Risk of Gestational Diabetes Mellitus: The DALI Lifestyle Pilot. Diabetes Care. 2015. 38:1650-6. doi:10.2337/dc15-0360.

521. Simmons, D, Devlieger, R, van Assche, A, Galjaard, S, Corcoy, R, Adelantado, JM, Dunne, F, Desoye, G, Kautzky-Willer, A, Damm, P, Mathiesen, ER, Jensen, DM, Andersen, LLT, Lapolla, A, Dalfra, MG, Bertolotto, A, Wender-Ozegowska, E, Zawiejska, A, Hill, D, Snoek, FJ. Association between Gestational Weight Gain, Gestational Diabetes Risk, and Obstetric Outcomes: A Randomized Controlled Trial Post Hoc Analysis. Nutrients. 2018. 10:1568. doi:10.3390/nu10111568.

522. Simoes-Wust, AP, Molto-Puigmarti, $C$, Jansen, EH, van Dongen, MC, Dagnelie, PC, Thijs, C. Organic food consumption during pregnancy and its association with health-related characteristics: the KOALA Birth Cohort Study. Public Health Nutr. 2017. 20:2145-2156. doi:10.1017/s1368980017001215.

523. Singh, SB, Madan, J, Coker, M, Hoen, A, Baker, ER, Karagas, MR, Mueller, NT. Does birth mode modify associations of maternal pre-pregnancy BMI and gestational weight gain with the infant gut microbiome? Int J Obes (Lond). 2019. doi:10.1038/s41366-0180273-0.

524. Singhal, A, Kennedy, K, Lanigan, J, Fewtrell, M, Cole, TJ, Stephenson, T, Elias-Jones, A, Weaver, LT, Ibhanesebhor, S, MacDonald, PD, Bindels, J, Lucas, A. Nutrition in infancy and long-term risk of obesity: evidence from 2 randomized controlled trials. Am J Clin Nutr. 2010. 92:1133-44. doi:10.3945/ajcn.2010.29302.

525. Skaug, MA, Helland, I, Solvoll, K, Saugstad, OD. Presence of ochratoxin A in human milk in relation to dietary intake. Food Addit Contam. 2001. 18:321-7. doi:10.1080/02652030117740.

526. Skreden, M, Bere, E, Sagedal, LR, Vistad, I, Overby, NC. Changes in fruit and vegetable consumption habits from pre-pregnancy to early pregnancy among Norwegian women. BMC Pregnancy Childbirth. 2017. 17:107. doi:10.1186/s12884-017-1291-y.

\section{Rationale}

Intervention/Exposure

Intervention/Exposure

Intervention/Exposure

Intervention/Exposure

Intervention/Exposure

Intervention/Exposure; Outcome

\section{Outcome}

Intervention/Exposure 
527. Skreden, M, Hillesund, ER, Wills, AK, Brantsaeter, AL, Bere, E, Overby, NC. Adherence to the New Nordic Diet during pregnancy and subsequent maternal weight development: a study conducted in the Norwegian Mother and Child Cohort Study (MoBa). Br J Nutr. 2018. 119:1286-1294. doi:10.1017/s0007114518000776.

528. Slane, JD, Levine, MD. Association of Restraint and Disinhibition to Gestational Weight Gain among Pregnant Former Smokers. Womens Health Issues. 2015. 25:390-5. doi:10.1016/j.whi.2015.03.005.

529. Sloan, NL, Lederman, SA, Leighton, J, Himes, JH, Rush, D. The effect of prenatal dietary protein intake on birth weight. Nutrition Research. 2001. 21:129-139. doi:10.1016/S02715317(00)00258-X.

530. Sommer, C, Sletner, L, Jenum, AK, Mrkrid, K, Andersen, LF, Birkeland, K, Mosdl, A. Ethnic differences in maternal dietary patterns are largely explained by socioeconomic score and integration score: a population-based study. Food \& Nutrition Research. 2013. 57:1-12. doi:10.3402/fnr.v57i0.21164.

531. Sotres-Alvarez, D, Herring, AH, Siega-Riz, AM. Latent transition models to study women's changing of dietary patterns from pregnancy to 1 year postpartum. Am J Epidemiol. 2013. 177:852-61. doi:10.1093/aje/kws303.

532. Spencer, L, Rollo, M, Hutchesson, M, Collins, C. Perceived healthy eating and physical activity factors influencing weight management in postpartum women: A mixed methods analysis. Obesity Research \& Clinical Practice. 2014. 8:96-96. doi:10.1016/j.orcp.2014.10.176.

533. Sridhar, SB, Darbinian, J, Ehrlich, SF, Markman, MA, Gunderson, EP, Ferrara, A, Hedderson, MM. Maternal gestational weight gain and offspring risk for childhood overweight or obesity. Am J Obstet Gynecol. 2014. 211:259.e1-8. doi:10.1016/j.ajog.2014.02.030.

534. Stendell-Hollis, NR, Laudermilk, MJ, West, JL, Thompson, PA, Thomson, CA. Recruitment of lactating women into a randomized dietary intervention: successful strategies and factors promoting enrollment and retention. Contemp Clin Trials. 2011. 32:505-11. doi:10.1016/j.cct.2011.03.007.

535. Stephens, TV, Woo, H, Innis, SM, Elango, R. Healthy pregnant women in Canada are consuming more dietary protein at 16- and 36-week gestation than currently recommended by the Dietary Reference Intakes, primarily from dairy food sources. Nutr Res. 2014. 34:569-76. doi:10.1016/j.nutres.2014.07.001.

536. Stuebe, AM, Oken, E, Gillman, MW. Associations of diet and physical activity during pregnancy with risk for excessive gestational weight gain. Am J Obstet Gynecol. 2009. 201:58.e1-8. doi:10.1016/j.ajog.2009.02.025.

Intervention/Exposure

Intervention/Exposure; Data collected in 1983

Study Design; Outcome

Outcome

Conference abstract

Intervention/Exposure; Outcome

Study Design; Outcome

Study Design; Intervention/Exposure

Intervention/Exposure 
537. Su, T, Lu, J, Ma, H. Lifestyle intervention prevents pregnant woman from gestational diabetes mellitus: a Chinese randomized controlled trial. International journal of clinical and experimental medicine. 2016. 9:23584-23590.

538. Suliga, E, Adamczyk-Gruszka, OK. Health behaviours of pregnant women and gestational weight gains -A pilot study. Medical Studies/Studia Medyczne. 2015. 31:161-167. doi:10.5114/ms.2015.54753.

539. Suliga, E, Rokita, W, Adamczyk-Gruszka, O, Pazera, G, Ciesla, E, Gluszek, S. Factors associated with gestational weight gain: a cross-sectional survey. BMC Pregnancy Childbirth. 2018. 18:465. doi:10.1186/s12884-018-2112-7.

540. Surendran, S, Aji, AS, Ariyasra, U, Sari, SR, Malik, SG, Tasrif, N, Yani, FF, Lovegrove, JA, Study Design Sudji, IR, Lipoeto, NI, Vimaleswaran, KS. A nutrigenetic approach for investigating the relationship between vitamin B12 status and metabolic traits in Indonesian women. $J$ Diabetes Metab Disord. 2019. 18(2):389-399. doi:10.1007/s40200-019-00424-z.

541. Swendeman, D, Comulada, WS, Koussa, M, Worthman, CM, Estrin, D, Rotheram-Borus, MJ, Ramanathan, N. Longitudinal Validity and Reliability of Brief Smartphone SelfMonitoring of Diet, Stress, and Physical Activity in a Diverse Sample of Mothers. JMIR Mhealth Uhealth. 2018. 6:e176. doi:10.2196/mhealth.9378.

542. Swensen, AR, Harnack, LJ, Ross, JA. Nutritional assessment of pregnant women enrolled in the Special Supplemental Program for Women, Infants, and Children (WIC). J Am Diet Assoc. 2001. 101:903-8. doi:10.1016/s0002-8223(01)00221-8.

543. Switkowski, KM, Jacques, PF, Must, A, Hivert, MF, Fleisch, A, Gillman, MW, RifasShiman, S, Oken, E. Higher Maternal Protein Intake during Pregnancy Is Associated with Lower Cord Blood Concentrations of Insulin-like Growth Factor (IGF)-II, IGF Binding Protein 3, and Insulin, but Not IGF-I, in a Cohort of Women with High Protein Intake. J Nutr. 2017. 147:1392-1400. doi:10.3945/jn.117.250589.

544. Szmeja, MA, Cramp, C, Grivell, RM, Deussen, AR, Yelland, LN, Dodd, JM. Use of a DVD to provide dietary and lifestyle information to pregnant women who are overweight or obese: a nested randomised trial. BMC Pregnancy Childbirth. 2014. 14:409. doi:10.1186/s12884-014-0409-8.

545. Tadayon, M, Khavayet, F, Abedi, P, Malehi, AS. The relationship between maternal lifestyle and children's body mass index: A cross-sectional study in Abadan, Iran. Journal of Midwifery \& Reproductive Health. 2019. 7:1824-1833. doi:10.22038/jmrh.2019.24445.1263.

546. Tahir, MJ, Haapala, JL, Foster, LP, Duncan, KM, Teague, AM, Kharbanda, EO, McGovern, PM, Whitaker, KM, Rasmussen, KM, Fields, DA, Jacobs, DR, Jr, Harnack, LJ, Demerath, EW. Higher Maternal Diet Quality during Pregnancy and Lactation Is Associated with Lower Infant Weight-For-Length, Body Fat Percent, and Fat Mass in Early Postnatal Life. Nutrients. 2019. 11. doi:10.3390/nu11030632.

Intervention/Exposure; Population

Study Design; Outcome

Intervention/Exposure; Outcome 
547. Talai Rad, N, Ritterath, C, Siegmund, T, Wascher, C, Siebert, G, Henrich, W, Buhling, KJ.

Outcome; Comparator Longitudinal analysis of changes in energy intake and macronutrient composition during pregnancy and 6 weeks post-partum. Arch Gynecol Obstet. 2011. 283:185-90. doi:10.1007/s00404-009-1328-1.

548. Tavares, MP, Devincenzi, MU, Sachs, A, de Vilhena Abrão, ACF. Nutritional status and diet quality of nursing mothers on exclusive breastfeeding. Acta Paulista de Enfermagem. 2013. 26:294-298.

549. Taveras, E, Blackburn, K, Gillman, M, Haines, J, McDonald, J, Price, S, Oken, E. First Steps for Mommy and Me: A Pilot Intervention to Improve Nutrition and Physical Activity Behaviors of Postpartum Mothers and Their Infants. Maternal \& Child Health Journal. 2011. 15:1217-1227. doi:10.1007/s10995-010-0696-2.

550. Teixeira, $\mathrm{VH}$, Moreira, $\mathrm{P}$. Maternal food intake and socioeconomic status to tackle childhood malnutrition. Jornal de Pediatria. 2016. 92:546-548. doi:10.1016/j.jped.2016.08.002.

551. Thomas Berube, L, Messito, MJ, Woolf, K, Deierlein, A, Gross, R. Correlates of Prenatal Diet Quality in Low-Income Hispanic Women. J Acad Nutr Diet. 2019. doi:10.1016/j.jand.2019.02.004.

552. Thomson, JL, Tussing-Humphreys, LM, Goodman, MH, Olender, S. Baseline Demographic, Anthropometric, Psychosocial, and Behavioral Characteristics of Rural, Southern Women in Early Pregnancy. Matern Child Health J. 2016. 20:1980-8. doi:10.1007/s10995-016-2016-y.

553. Thomson, JL, Tussing-Humphreys, LM, Goodman, MH, Olender, SE. Gestational Weight Gain: Results from the Delta Healthy Sprouts Comparative Impact Trial. J Pregnancy. 2016. 2016:5703607. doi:10.1155/2016/5703607.

554. Thomson, JL, Tussing-Humphreys, LM, Goodman, MH. Delta Healthy Sprouts: a randomized comparative effectiveness trial to promote maternal weight control and reduce childhood obesity in the Mississippi Delta. Contemp Clin Trials. 2014. 38:82-91. doi:10.1016/j.cct.2014.03.004.

555. Tian, HM, Wu, YX, Lin, YQ, Chen, $X Y, Y u, M, L u, T$, Xie, L. Dietary patterns affect maternal macronutrient intake levels and the fatty acid profile of breast milk in lactating Chinese mothers. Nutrition. 2019. 58:83-88. doi:10.1016/j.nut.2018.06.009.

556. Tielemans, MJ, Erler, NS, Franco, OH, Jaddoe, VWV, Steegers, EAP, Kiefte-de jong, JC. Dietary acid load and blood pressure development in pregnancy: The Generation R Study. Clinical Nutrition. 2018. 37:597-603. doi:10.1016/j.clnu.2017.01.013.

Intervention/Exposure

\section{Study Design; Outcome}

Intervention/Exposure; Outcome

Editorial

Study Design; Outcome

Study Design

Study Design; No Results

Study Design

Outcome; Association btw DP and GWG not analyzed 
557. Timmermans, S, Steegers-Theunissen, RP, Vujkovic, M, den Breeijen, H, Russcher, $H$, Lindemans, J, Mackenbach, J, Hofman, A, Lesaffre, EE, Jaddoe, VV, Steegers, EA. The Mediterranean diet and fetal size parameters: the Generation R Study. Br J Nutr. 2012. 108:1399-409. doi:10.1017/s000711451100691x.

558. Tobias, DK, Hu, FB, Chavarro, J, Rosner, B, Mozaffarian, D, Zhang, C. Healthful dietary patterns and type 2 diabetes mellitus risk among women with a history of gestational diabetes mellitus. Arch Intern Med. 2012. 172:1566-72.

doi:10.1001/archinternmed.2012.3747.

559. Torjusen, H, Brantsaeter, AL, Haugen, M, Lieblein, G, Stigum, H, Roos, G, HolmboeOttesen, G, Meltzer, HM. Characteristics associated with organic food consumption during pregnancy; data from a large cohort of pregnant women in Norway. BMC Public Health. 2010. 10:775. doi:10.1186/1471-2458-10-775.

560. Tovar, A, Guthrie, LB, Platek, D, Stuebe, A, Herring, SJ, Oken, E. Modifiable predictors associated with having a gestational weight gain goal. Matern Child Health J. 2011. 15:1119-26. doi:10.1007/s10995-010-0659-7.

561. Tovar, A, Kaar, JL, McCurdy, K, Field, AE, Dabelea, D, Vadiveloo, M. Maternal vegetable Intervention/Exposure; Outcome intake during and after pregnancy. BMC Pregnancy Childbirth. 2019. 19:267. doi:10.1186/s12884-019-2353-0.

562. Tovar, A, Must, A, Bermudez, OI, Hyatt, RR, Chasan-Taber, L. The impact of gestational weight gain and diet on abnormal glucose tolerance during pregnancy in Hispanic women. Matern Child Health J. 2009. 13:520-30. doi:10.1007/s10995-008-0381-x.

563. Trak-Fellermeier, MA, Campos, M, Melendez, M, Pomeroy, J, Palacios, C, Rivera-Vinas, J, Mendez, K, Febo, I, Willett, W, Gillman, MW, Franks, PW, Joshipura, K. PEARLS randomized lifestyle trial in pregnant Hispanic women with overweight/obesity: gestational weight gain and offspring birthweight. Diabetes Metab Syndr Obes. 2019. 12:225-238. doi:10.2147/dmso.S179009.

564. Trude, A, Black, M, Hurley, K, Ojeda, LC, Wang, Y. Maternal Anxiety Symptoms and Mother-toddler Diet Quality Among WIC Participants (OR03-08-19). Curr Dev Nutr. 2019. 3. doi:10.1093/cdn/nzz048.OR03-08-19.

565. Tryggvadottir, EA, Medek, H, Birgisdottir, BE, Geirsson, RT, Gunnarsdottir, I. Association between healthy maternal dietary pattern and risk for gestational diabetes mellitus. Eur $\mathrm{J}$ Clin Nutr. 2016. 70:237-42. doi:10.1038/ejcn.2015.145.

566. Turner, RE, Langkamp-Henken, B, Littell, RC, Lukowski, MJ, Suarez, MF. Comparing nutrient intake from food to the estimated average requirements shows middle- to upperincome pregnant women lack iron and possibly magnesium. Journal of the American Dietetic Association. 2003. 103:461-466.

Intervention/Exposure

Intervention/Exposure

\section{Outcome}

Population; Outcome

Outcome

Intervention/Exposure; Outcome

Abstract

\section{Outcome}

Intervention/Exposure 
567. Tussing-Humphreys, LM, Thomson, JL, Goodman, MH, Olender, S. Maternal diet quality and nutrient intake in the gestational period: results from the delta healthy sprouts comparative impact trial. Matern Health Neonatol Perinatol. 2016. 2:8. doi:10.1186/s40748-016-0036-7.

568. Uusitalo, U, Arkkola, T, Ovaskainen, ML, Kronberg-Kippila, C, Kenward, MG, Veijola, R, Simell, O, Knip, M, Virtanen, SM. Unhealthy dietary patterns are associated with weight gain during pregnancy among Finnish women. Public Health Nutr. 2009. 12:2392-9. doi:10.1017/s136898000900528x.

569. Vahamiko, S, Isolauri, E, Laitinen, $\mathrm{K}$. Weight status and dietary intake determine serum leptin concentrations in pregnant and lactating women and their infants. Br J Nutr. 2013. 110:1098-106. doi:10.1017/s0007114513000214.

570. Vahamiko, S, Isolauri, E, Pesonen, U, Koskinen, P, Ekblad, U, Laitinen, K. Dietary sucrose intake is related to serum leptin concentration in overweight pregnant women. Eur J Nutr. 2010. 49:83-90. doi:10.1007/s00394-009-0052-8.

571. Valkama, A, Koivusalo, S, Lindstrom, J, Meinila, J, Kautiainen, H, Stach-Lempinen, B, Rono, K, Klemetti, M, Poyhonen-Alho, M, Tiitinen, A, Huvinen, E, Laivuori, H, Andersson, $S$, Roine, R, Eriksson, JG. The effect of dietary counselling on food intakes in pregnant women at risk for gestational diabetes: a secondary analysis of a randomised controlled trial RADIEL. Eur J Clin Nutr. 2016. 70:912-7. doi:10.1038/ejcn.2015.205.

572. Valkama, AJ, Meinila, JM, Koivusalo, SB, Lindstrom, J, Rono, K, Stach-Lempinen, B, Eriksson, JG. Diet quality as assessed by the Healthy Food Intake Index and relationship with serum lipoprotein particles and serum fatty acids in pregnant women at increased risk for gestational diabetes. Br J Nutr. 2018. 120:914-924. doi:10.1017/s0007114518002404.

573. van der Wijden, CL, Steinbach, S, van der Ploeg, HP, van Mechelen, W, van Poppel, MN . A longitudinal study on the relationship between eating style and gestational weight gain. Appetite. 2014. 83:304-8. doi:10.1016/j.appet.2014.09.001.

574. van Elten, TM, Karsten, MDA, Geelen, A, Gemke, Rjbj, Groen, H, Hoek, A, van Poppel, MNM, Roseboom, TJ. Preconception lifestyle intervention reduces long term energy intake in women with obesity and infertility: a randomised controlled trial. Int $\mathrm{J}$ Behav Nutr Phys Act. 2019. 16:3. doi:10.1186/s12966-018-0761-6.

575. van Poppel, MNM, Jelsma, JGM, Simmons, D, Devlieger, R, Jans, G, Galjaard, S, Corcoy, R, Adelantado, JM, Dunne, F, Harreiter, J, Kautzky-Willer, A, Damm, P, Mathiesen, ER, Jensen, DM, Andersen, LL, Tanvig, M, Lapolla, A, Dalfra, MG, Bertolotto, A, WenderOzegowska, E, Zawiejska, A, Hill, D, Desoye, G, Snoek F J. Mediators of lifestyle behaviour changes in obese pregnant women. Secondary analyses from the DALI lifestyle randomised controlled trial. Nutrients. 2019. 11. doi:10.3390/nu11020311.

\section{Intervention/Exposure}

Intervention/Exposure

Intervention/Exposure; Outcome

\section{Outcome}

Intervention/Exposure

Intervention/Exposure; Population

Outcome 
576. Vander Wyst, KB, Vercelli, ME, O'Brien, KO, Cooper, EM, Pressman, EK, Whisner, CM. A social media intervention to improve nutrition knowledge and behaviors of low income, pregnant adolescents and adult women. PLoS One. 2019. 14:e0223120. doi:10.1371/journal.pone.0223120.

577. Vandyousefi, S, Whaley, S, Asigbee, F, Landry, M, Ghaddar, R, Davis, J. Association of Breastfeeding and Sugar-Sweetened Beverage Consumption with Obesity Prevalence in Offspring Born to Mothers with and Without Gestational Diabetes Mellitus (P11-098-19). Curr Dev Nutr. 2019. 3. doi:10.1093/cdn/nzz048.P11-098-19.

578. Vega-Lopez, S, Pignotti, GA, Todd, M, Keller, C. Egg Intake and Dietary Quality among Overweight and Obese Mexican-American Postpartum Women. Nutrients. 2015. 7:840212. doi:10.3390/nu7105402.

579. Ventegodt, S, Flensborg-Madsen, T, Andersen, NJ, Merrick, J. Which factors determine our quality of life, health and ability? Results from a Danish population sample and the Copenhagen Perinatal Cohort. Journal of the College of Physicians and Surgeons Pakistan. 2008. 18:445-450. doi:10.1017/BJN20061929.

580. Vesco, K, Leo, M, Gillman, M, King, J, McEvoy, C, Karanjaa, N, Perrin, N, Eckhardt, C, Smith, KS, Stevens, V. Impact of a weight management intervention on pregnancy outcomes among obese women: the Healthy Moms Trial. American journal of obstetrics and gynecology. 2013. 208:S352. doi:10.1016/j.ajog.2012.12.009.

581. Vesco, KK, Karanja, N, King, JC, Gillman, MW, Leo, MC, Perrin, N, McEvoy, CT, Eckhardt, CL, Smith, KS, Stevens, VJ. Efficacy of a group-based dietary intervention for limiting gestational weight gain among obese women: a randomized trial. Obesity (Silver Spring). 2014. 22:1989-96. doi:10.1002/oby.20831.

582. Vieten, C, Laraia, BA, Kristeller, J, Adler, N, Coleman-Phox, K, Bush, NR, Wahbeh, H, Duncan, LG, Epel, E. The mindful moms training: development of a mindfulness-based intervention to reduce stress and overeating during pregnancy. BMC Pregnancy Childbirth. 2018. 18:201. doi:10.1186/s12884-018-1757-6.

583. Vilela, AA, Pinto Tde, J, Rebelo, F, Benaim, C, Lepsch, J, Dias-Silva, CH, Castro, MB Kac, G. Association of Prepregnancy Dietary Patterns and Anxiety Symptoms from Midpregnancy to Early Postpartum in a Prospective Cohort of Brazilian Women. J Acad Nutr Diet. 2015. 115:1626-35. doi:10.1016/j.jand.2015.01.007.

584. Villar-Vidal, M, Amiano, $P$, Rodriguez-Bernal, C, Santa Marina, L, Mozo, I, Vioque, J, Navarrete-Munoz, EM, Romaguera, D, Valvi, D, Fernandez Samoano, A, Tardon, A, Ibarluzea, J. Compliance of nutritional recommendations of Spanish pregnant women according to sociodemographic and lifestyle characteristics: a cohort study. Nutr Hosp 2015. 31:1803-12. doi:10.3305/nh.2015.31.4.8293.

\section{Abstract}

Outcome

Study Design; Outcome

Abstract

Intervention/Exposure

Intervention/Exposure; Outcome

Outcome

Study Design 
585. Vinter, CA, Jensen, DM, Ovesen, $P$, Beck-Nielsen, H, Tanvig, M, Lamont, RF, Jorgensen,

Intervention/Exposure

JS. Postpartum weight retention and breastfeeding among obese women from the randomized controlled Lifestyle in Pregnancy (LiP) trial. Acta Obstet Gynecol Scand. 2014. 93:794-801. doi:10.1111/aogs.12429.

586. Vinter, CA, Jørgensen, JS, Ovesen, $P$, Beck-Nielsen, H, Skytthe, A, Jensen, DM. Metabolic effects of lifestyle intervention in obese pregnant women. Results from the randomized controlled trial 'Lifestyle in Pregnancy' (LiP). Diabetic Medicine. 2014. 31:1323-1330. doi:10.1111/dme.12548.

587. Vlaardingerbroek, $H$, Roelants, JA, Rook, D, Dorst, K, Schierbeek, H, Vermes, A, Vermeulen, MJ, van Goudoever, JB, van den Akker, $\mathrm{CH}$. Adaptive regulation of amino acid metabolism on early parenteral lipid and high-dose amino acid administration in VLBW infants - a randomized, controlled trial. Clin Nutr. 2014. 33:982-90. doi:10.1016/j.clnu.2014.01.002.

588. von Ruesten, A, Brantsaeter, AL, Haugen, M, Meltzer, HM, Mehlig, K, Winkvist, A, Lissner, $\mathrm{L}$. Adherence of pregnant women to Nordic dietary guidelines in relation to postpartum weight retention: results from the Norwegian Mother and Child Cohort Study. BMC Public Health. 2014. 14:75. doi:10.1186/1471-2458-14-75.

589. Wadhwa, EL, Ma, C, Shaw, GM, Carmichael, SL. Gastroschisis and maternal intake of phytoestrogens. Am J Med Genet A. 2016. 170:2078-82. doi:10.1002/ajmg.a.37659.

590. Walker, LO, Kang, S, Sterling B S. Weight-Loss Resilience Among Low-Income Postpartum Women: Association With Health Habits. West J Nurs Res. 2019. :193945918824598. doi:10.1177/0193945918824598.

591. Wall, CR, Gammon, CS, Bandara, DK, Grant, CC, Atatoa Carr, PE, Morton, SM. Dietary Patterns in Pregnancy in New Zealand-Influence of Maternal Socio-Demographic, Health and Lifestyle Factors. Nutrients. 2016. 8. doi:10.3390/nu8050300.

592. Walsh, J, Mahony, R, Foley, M, Mc Auliffe, F. A randomised control trial of low glycaemic index carbohydrate diet versus no dietary intervention in the prevention of recurrence of macrosomia. BMC Pregnancy Childbirth. 2010. 10:16. doi:10.1186/1471-2393-10-16.

593. Walsh, J, McGowan, C, Byrne, J, Foley, M, Mahony, R, McAuliffe, F. The influence of a low glycaemic index dietary intervention on maternal glycaemic index, dietary intake and gestational weight gain. American journal of obstetrics and gynecology. 2013. 208:S33. doi:10.1016/j.ajog.2012.10.228.

594. Walsh, JM, Mahony, RM, Canty, G, Foley, ME, McAuliffe, FM. Identification of those most likely to benefit from a low-glycaemic index dietary intervention in pregnancy. Br J Nutr. 2014. 112:583-9. doi:10.1017/s000711451400110x.

Population

Study Design; Intervention/Exposure

Intervention/Exposure

\section{Outcome}

Study Design; Study protocol

Abstract

Intervention/Exposure 
595. Walsh, JM, Mahony, RM, Culliton, M, Foley, ME, McAuliffe, FM. Impact of a low glycemic index diet in pregnancy on markers of maternal and fetal metabolism and inflammation. Reprod Sci. 2014. 21:1378-81. doi:10.1177/1933719114525275.

596. Walsh, JM, McGowan, CA, Mahony, R, Foley, ME, McAuliffe, FM. Low glycaemic index diet in pregnancy to prevent macrosomia (ROLO study): randomised control trial. Bmj. 2012. 345:e5605. doi:10.1136/bmj.e5605.

597. Wand, H, Ramjee, G. High prevalence of obesity among women who enrolled in HIV prevention trials in KwaZulu-Natal, South Africa: healthy diet and life style messages should be integrated into HIV prevention programs. BMC Public Health. 2013. 13:159159. doi:10.1186/1471-2458-13-159.

598. Wang, C, Gao, J, Liu, N, Yu, S, Qiu, L, Wang, D. Maternal factors associated with neonatal vitamin D deficiency. J Pediatr Endocrinol Metab. 2019. 32:167-172. doi:10.1515/jpem-2018-0422.

599. Wang, L, Liu, H, Zhang, S, Leng, J, Liu, G, Zhang, C, Li, WQ, Li, N, Li, W, Li, Y, Sun, S, Yu, Z, Yang, X, Hu, G. Obesity index and the risk of diabetes among Chinese women with prior gestational diabetes. Diabetic Medicine. 2014. 31:1368-1377. doi:10.1111/dme.12532.

600. Wang, L, Dalton, WT, 3rd, Schetzina, KE, Fulton-Robinson, H, Holt, N, Ho, A, Tudiver, F, $\mathrm{Wu}, \mathrm{T}$. Home food environment, dietary intake, and weight among overweight and obese children in Southern Appalachia. Southern Medical Journal. 2013. 106:550-557. doi:10.1097/SMJ.0000000000000008.

601. Wansink, B. Project M.O.M.: Mothers \& Others \& MyPyramid. J Am Diet Assoc. 2008. 108:1302-4. doi:10.1016/j.jada.2008.06.444.

602. Watson, PE, McDonald, BW. Major influences on nutrient intake in pregnant New Zealand women. Maternal \& Child Health Journal. 2009. 13:695-706. doi:10.1007/s10995-0080405-6.

603. Watson, PE, McDonald, BW. Seasonal variation of nutrient intake in pregnancy: effects on infant measures and possible influence on diseases related to season of birth. Eur $\mathrm{J} \mathrm{Clin}$ Nutr. 2007. 61:1271-80. doi:10.1038/sj.ejcn.1602644.

604. Watson, PE, McDonald, BW. The association of maternal diet and dietary supplement intake in pregnant New Zealand women with infant birthweight. Eur J Clin Nutr. 2010. 64:184-93. doi:10.1038/ejcn.2009.134.

605. Wen, LM, Flood, VM, Simpson, JM, Rissel, C, Baur, LA. Dietary behaviours during pregnancy: findings from first-time mothers in southwest Sydney, Australia. International Journal of Behavioral Nutrition \& Physical Activity. 2010. 7:7p-7p. doi:10.1186/1479-58687-13.

\section{Outcome}

Intervention/Exposure; Comparator 
606. Wen, LM, Simpson, JM, Rissel, C, Baur, LA. Maternal "junk food" diet during pregnancy as a predictor of high birthweight: findings from the healthy beginnings trial. Birth. 2013 . 40:46-51. doi:10.1111/birt.12028.

607. Whisner, CM, Young, BE, Pressman, EK, Queenan, RA, Cooper, EM, O'Brien, KO. Maternal diet but not gestational weight gain predicts central adiposity accretion in utero among pregnant adolescents. Int J Obes (Lond). 2015. 39:565-70. doi:10.1038/ijo.2014.202.

608. White, SL, Flynn, AC, Poston, L. Impact of a positive or negative diagnosis of gestational diabetes and treatment, on weight change and dietary behaviour in an obese cohort: secondary analysis of the UK pregnancies better eating and activity trial (UPBEAT) randomised controlled trial (RCT). Diabetic medicine. 2019. 36:65-. doi:10.1111/dme.13883.

609. Wilkinson, SA, McIntyre, HD. Evaluation of the 'healthy start to pregnancy' early antenatal Outcome health promotion workshop: a randomized controlled trial. BMC Pregnancy Childbirth. 2012. 12:131. doi:10.1186/1471-2393-12-131.

610. Wilkinson, SA, van der Pligt, $P$, Gibbons, KS, McIntyre, HD. Trial for Reducing Weight Retention in New Mums: a randomised controlled trial evaluating a low intensity, postpartum weight management programme. J Hum Nutr Diet. 2015. 28 Suppl 1:15-28. doi:10.1111/jhn.12193.

611. Willcox, JC, Wilkinson, SA, Lappas, M, Ball, K, Crawford, D, McCarthy, EA, Fjeldsoe, B, Whittaker, R, Maddison, R, Campbell, KJ. A mobile health intervention promoting healthy gestational weight gain for women entering pregnancy at a high body mass index: the txt4two pilot randomised controlled trial. Bjog. 2017. 124:1718-1728. doi:10.1111/1471. 0528.14552 .

612. Williams, JE, Carrothers, JM, Lackey, KA, Beatty, NF, York, MA, Brooker, SL, Shafii, B, Price, WJ, Settles, ML, McGuire, MA, McGuire, MK. Human Milk Microbial Community Structure Is Relatively Stable and Related to Variations in Macronutrient and Micronutrient Intakes in Healthy Lactating Women. J Nutr. 2017. 147:1739-1748. doi:10.3945/jn.117.248864.

613. Wiltheiss, GA, Lovelady, CA, West, DG, Brouwer, RJ, Krause, KM, Ostbye, T. Diet quality Population and weight change among overweight and obese postpartum women enrolled in a behavioral intervention program. J Acad Nutr Diet. 2013. 113:54-62. doi:10.1016/j.jand.2012.08.012.

614. Winkvist, A, Bertz, F, Ellegard, L, Bosaeus, I, Brekke, HK. Metabolic risk profile among overweight and obese lactating women in Sweden. PLoS One. 2013. 8:e63629. doi:10.1371/journal.pone.0063629.

615. Worawong, C. A nutrition intervention focused on goals of Thai pregnant women. 2008. :221 p-221 p. 
616. Wyst, KV, Buman, M, Shaibi, G, Petrov, M, Reifsnider, E, Whisner, C. Evaluation of Variability in Resting Energy Expenditure and Its Relationship with Macronutrients and Gestational Weight Gain During the Second Trimester of Pregnancy (P11-136-19). Curr Dev Nutr. 2019. 3. doi:10.1093/cdn/nzz048.P11-136-19.

617. Xu, Q, Gao, ZY, Li, LM, Wang, L, Zhang, Q, Teng, Y, Zhao, X, Ge, S, Jing, HJ, Yang, YT, Liu, XJ, Lyu, CJ, Mao, L, Yu, XM, Liu, YH, Kong, AJ, Yang, XY, Liu, Z, Zhang, Y, Wang, J, Zhang, XS, Xue, CY, Lu, YP. The Association of Maternal Body Composition and Dietary Intake with the Risk of Gestational Diabetes Mellitus during the Second Trimester in a Cohort of Chinese Pregnant Women. Biomed Environ Sci. 2016. 29:1-11. doi:10.3967/bes2016.001

618. Xuto, Piyanut, Sinsuksai, Nittaya, Piaseu, Noppawan, Nityasuddhi, Dechavush, Phupong, Vorapong. A Causal Model of Postpartum Weight Retention among Thais. Pacific Rim International Journal of Nursing Research. 2012. 16:48-63.

619. Yao, J, Cong, L, Zhu, B, Wang, T. Effect of dietary approaches to stop hypertension diet plan on pregnancy outcome patients with gestational diabetes mellitus. Bangladesh Journal of Pharmacology. 2015. 10:732-738. doi:10.3329/bjp.v10i4.23813.

620. Yin, J, Quinn, S, Dwyer, T, Ponsonby, AL, Jones, G. Maternal diet, breastfeeding and adolescent body composition: a 16-year prospective study. Eur J Clin Nutr. 2012. 66:1329-34. doi:10.1038/ejcn.2012.122.

621. Yisahak, S, Hinkle, S, Mumford, S, Li, M, Andriessen, V, Grantz, K, Zhang, C, Grewal, J. Association of Maternal Vegetarian Diets with Neonatal Anthropometry in the NICHD Fetal Growth Study (OR35-08-19). Curr Dev Nutr. 2019. 3. doi:10.1093/cdn/nzz048.OR35-0819.

622. Yong, HY, Shariff, ZM, Mohd Yusof, BN, Rejali, Z, Bindels, J, Tee, YYS, van der Beek, EM. Associations between the dietary patterns of pregnant Malaysian women and ethnicity, education, and early pregnancy waist circumference: A prospective cohort study. Nutr Res Pract. 2019. 13:230-239. doi:10.4162/nrp.2019.13.3.230.

623. Yusuf, $\mathrm{H}$, Subih, HS, Obeidat, BS, Sharkas, G. Associations of macro and micronutrients and antioxidants intakes with preeclampsia: A case-control study in Jordanian pregnant women. Nutr Metab Cardiovasc Dis. 2019. 29:458-466. doi:10.1016/j.numecd.2019.01.008.

624. Zambrano, E, Nathanielsz, PW. Relative contributions of maternal Western-type high fat, high sugar diets and maternal obesity to altered metabolic function in pregnancy. Journal of Physiology. 2017. 595:4573-4574. doi:10.1113/JP274392.

625. Zareei, S, Homayounfar, R, Naghizadeh, MM, Ehrampoush, E, Rahimi, M. Dietary pattern in pregnancy and risk of gestational diabetes mellitus (GDM). Diabetes Metab Syndr. 2018. 12:399-404. doi:10.1016/j.dsx.2018.03.004 
626. Zeng, L, Yan, H, Cheng, Y, Dibley, MJ. Modifying effects of wealth on the response to nutrient supplementation in pregnancy on birth weight, duration of gestation, and perinatal mortality in rural western china: double-blind cluster randomized controlled trial. Obstetrical \& gynecological survey. 2011. 66:477-478. doi:10.1097/OGX.0b013e31823520d5.

627. Zhang, C, Liu, S, Solomon, CG, Hu, FB. Dietary fiber intake, dietary glycemic load, and the risk for gestational diabetes mellitus. Diabetes Care. 2006. 29:2223-2230.

628. Zhang, C, Schulze, MB, Solomon, CG, Hu, FB. A prospective study of dietary patterns, meat intake and the risk of gestational diabetes mellitus. Diabetologia. 2006. 49:2604-13. doi:10.1007/s00125-006-0422-1.

629. Zhang, C, Tobias, DK, Chavarro, JE, Bao, W, Wang, D, Ley, SH, Hu, FB. Adherence to healthy lifestyle and risk of gestational diabetes mellitus: prospective cohort study. Bmj. 2014. 349:g5450. doi:10.1136/bmj.g5450.

630. Zielinska, MA, Hamulka, J, Wesolowska, A. Carotenoid Content in Breastmilk in the 3rd and 6th Month of Lactation and Its Associations with Maternal Dietary Intake and Anthropometric Characteristics. Nutrients. 2019. 11. doi:10.3390/nu11010193.

631. Zulyniak, MA, de Souza, RJ, Shaikh, M, Desai, D, Lefebvre, DL, Gupta, M, Wilson, J, Wahi, G, Subbarao, P, Becker, AB, Mandhane, P, Turvey, SE, Beyene, J, Atkinson, S, Morrison, KM, McDonald, S, Teo, KK, Sears, MR, Anand, SS. Does the impact of a plantbased diet during pregnancy on birth weight differ by ethnicity? A dietary pattern analysis from a prospective Canadian birth cohort alliance. BMJ Open. 2017. 7:e017753. doi:10.1136/bmjopen-2017-017753.

\section{Outcome}

Intervention/Exposure; Population 